\title{
NOVEL OPTICAL ENDOSCOPES FOR EARLY CANCER DIAGNOSIS AND THERAPY
}
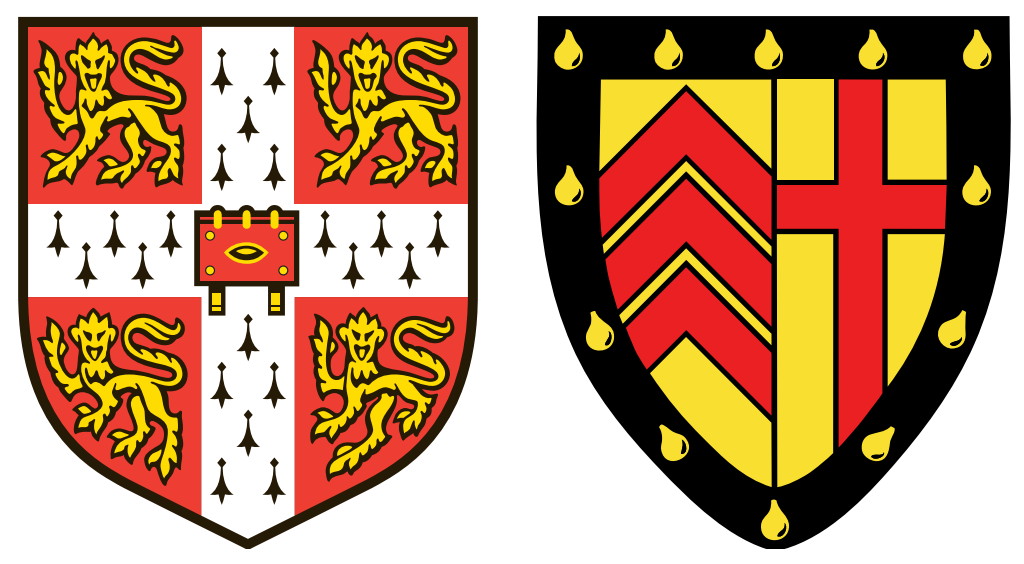

\section{Dale Jonathan Waterhouse}

Clare College

Supervisor: Dr Sarah E. Bohndiek

Department of Physics and CRUK Cambridge Institute

University of Cambridge

This dissertation is submitted for the degree of

Doctor of Philosophy

September 2018 



\section{SUMMARY}

\section{NOVEL OPTICAL ENDOSCOPES FOR EARLY CANCER DIAGNOSIS AND THERAPY}

\section{Dale Jonathan Waterhouse}

Imaging is the only medical tool currently capable of non-invasively capturing detailed, real time and spatially resolved biochemical information in vivo and thus delineating disease so that non-invasive curative resection or treatment of the affected area can take place. Though visible and near infrared (NIR) light undergo a wide range of complex interactions in tissue interactions which can be harnessed to yield useful information about the underlying pathology - optical imaging has yet to be fully utilised in clinic, with many existing techniques relying on standard colour imaging that replicates human vision.

This thesis describes my recent effort advancing novel optical endoscopic imaging techniques towards clinical translation. Before embarking on the development of novel devices, an analysis was made of the common challenges in translating optical imaging techniques. Through this work, a streamlined roadmap to clinical translation was developed, and key translational characteristics were defined. These were used to guide subsequent development of endoscopic devices.

Initial efforts were focused on the development of flexible endoscopes for detection of dysplasia in Barrett's oesophagus. To enable molecular imaging with a newly discovered targeted fluorescent contrast agent, a bimodal endoscope capable of capturing NIR fluorescence and white light reflectance was developed around a clinically translatable device architecture, and image artefacts were addressed by developing and evaluating image correction algorithms. This technique demonstrated significant potential for delineation of dysplasia in ex vivo samples. Next, a multispectral endoscope capable of imaging multiple fluorophores or endogenous tissue reflectance was developed. This device was successfully translated to a clinical pilot study, where initial results showed the promising potential of multispectral endoscopy for delineation of dysplasia based on endogenous reflectance from oesophageal tissue. Finally, multispectral imaging was explored for intraoperative delineation of adenoma and healthy pituitary tissue. A novel rigid multispectral endoscope was developed, preliminary technical characterisation of this device was performed, and a clinical pilot study was planned.

With continuation of this work as outlined at the end of this thesis, the novel techniques described here have the potential to improve the standard of care in their respective indications. 


\section{DECLARATION}

This dissertation is the result of my own work and includes nothing which is the outcome of work done in collaboration except where specifically indicated in the text. It has not been previously submitted, in part or whole, to any university of institution for any degree, diploma, or other qualification.

In accordance with the Department of Physics guidelines, this thesis is does not exceed 60,000 words, including summary/abstract, tables, footnotes and appendices, but excluding table of contents, photographs, diagrams, figure captions, list of figures/diagrams, list of abbreviations/acronyms, bibliography and acknowledgements.

Dale Jonathan Waterhouse

September 2018 
To my mum and dad,

whose love gave me the strength to succeed, and to my brother Aaron,

my best friend 


\section{ACKNOWLEDGEMENTS}

First, I would like to thank my supervisor, Sarah Bohndiek, for giving me the opportunity to join VISIONLab in 2014. I could not have asked for a better supervisor. Throughout my time in her lab, Sarah made time to provide scientific advice and feedback and supported me in purchasing equipment, in travelling to conferences and in my professional development. Despite the lab expanding rapidly, Sarah's schedule, along with her office door, have remained open. Her openness, efficiency, and the apparent ease with which she carries out her work is inspirational and these qualities have cultivated a similarly efficient yet relaxed atmosphere in VISIONLab. Never have I felt the crippling pressure of my supervisor bearing down upon me, a feeling all too often described by my peers.

Still, experiments failed. Equipment broke. Code crashed. During these times, I am grateful to have been surrounded by supportive colleagues. As a novice to research I am thankful that James Joseph and George Gordon were so willing to show me the ropes. I would also like to acknowledge the support of my exceptional clinical collaborators, especially Massimiliano di Pietro, Wladyslaw Januszewicz and James Tysome, whose support and patience has helped facilitate clinical translation of my work. I am grateful to have worked alongside Siri Luthman, tackling our problems together in an otherwise desolate optics lab.

I would also like to thank my colleagues turned close friends. To Isa, James and Judith, for conversations over coffee, in the car and over beers. Your warm words were always reassuring. To Abby, for helping me to keep calm, and for reading and correcting this thesis. To Michal, for all the fun we shared over the years. Further thanks go to my friends in college, especially Andrea, for the relaxing evenings watching trash TV, and Alexis, for the formals and nightsout which made the end of the week worth looking forward to. And a huge thank you to Lina, for her love and support in the final stretch.

Sadly, my big nan-nan is not here to see me complete my thesis. She is dearly missed and I am sure she would be immensely proud, as are my other grandparents. Their pride and love have spurred me on throughout my research. I express immense gratitude to my mum, dad and brother, Aaron. The weekends we spent in Cambridge, and at home, helped to remind me of the world outside the Cambridge bubble. Even in their absence, their love, support and advice stayed with me every step of the way, strengthening me in challenging times. The lessons my parents have taught me, and the example they set, have shaped all aspects of who I am and where I am today, and I will be forever grateful.

Finally, I would like to acknowledge the funding from the CRUK-EPSRC Cancer Imaging Centre in Cambridge and Manchester, without which I could not have carried out this work. These thanks extend to the millions of people whose generous donations ensure this work continues. 


\section{CONTENTS}

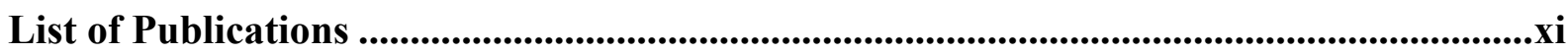

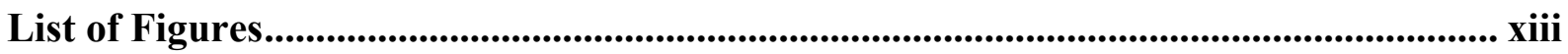

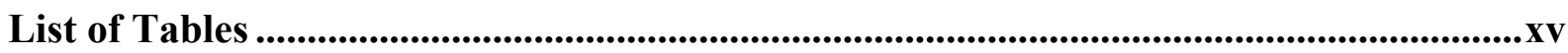

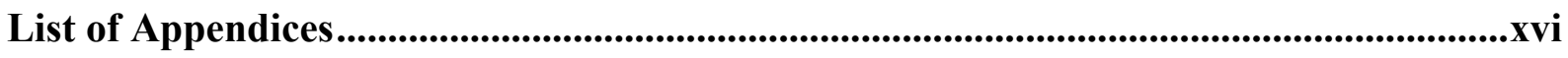

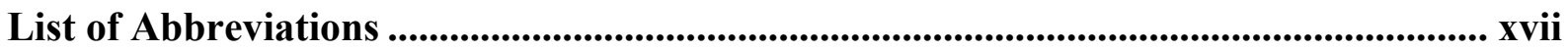

1 Translation of Optical Imaging Techniques............................................................................1

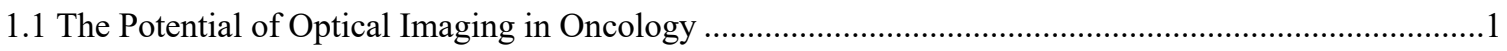

1.2 Challenges of Clinical Translation of Optical Imaging Techniques ........................................................

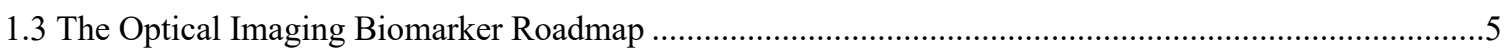

1.3.1 Barriers to Translation of Optical Imaging Techniques .............................................................

1.3.2 Translational Characteristics of Optical Imaging Techniques...................................................... 10

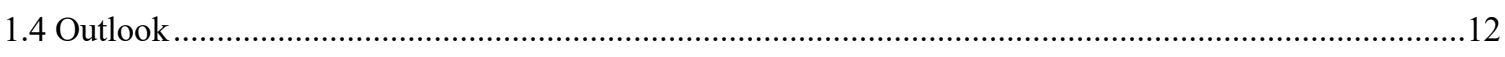

2 Flexible Endoscopy: Early Detection of Dysplasia in Barrett's Oesophagus..................15

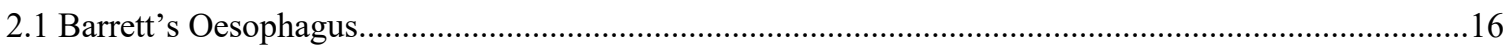

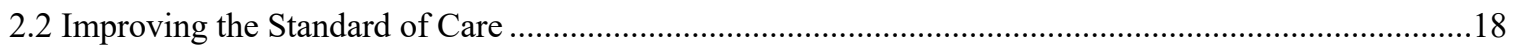

2.2.1 Categories of Advanced Optical Endoscopic Techniques ..........................................................18

2.2.2 Advanced Optical Endoscopic Techniques in Clinical Practice for Barrett's Surveillance ..........18

2.2.3 Emerging Optical Endoscopic Techniques for Barrett's Surveillance .........................................23

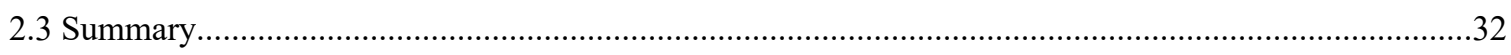

3 Flexible Endoscopy: Device Architecture ...................................................................................35

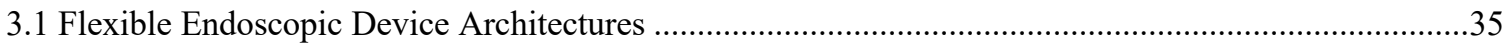

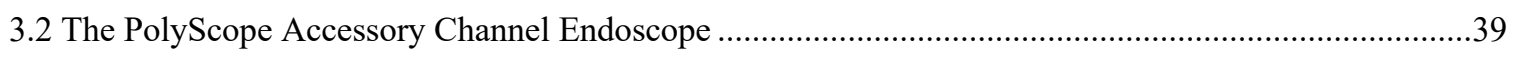

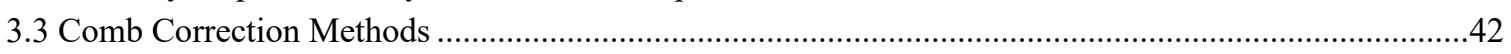

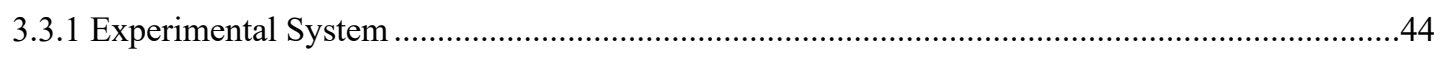

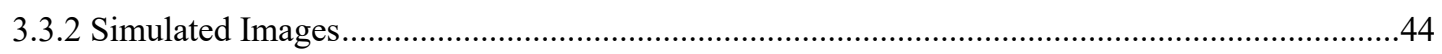

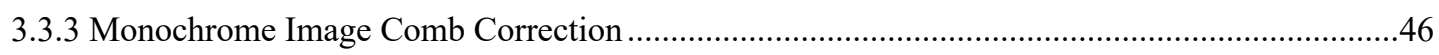

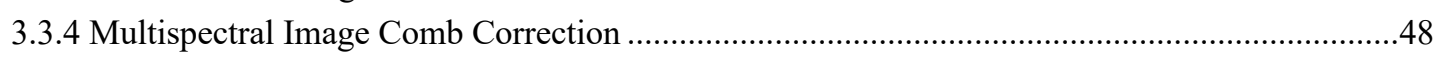

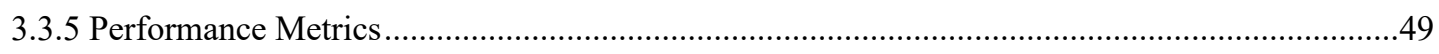

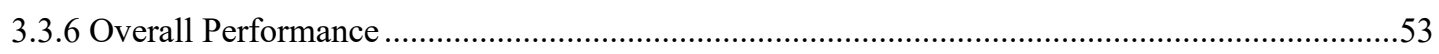

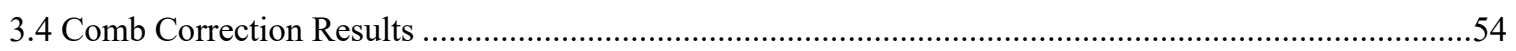

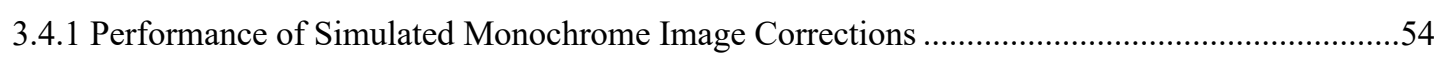

3.4.2 Performance of Experimentally Captured Monochrome Image Corrections .................................55

3.4.3 Performance of Captured Multispectral Image Corrections ……………………………….........60

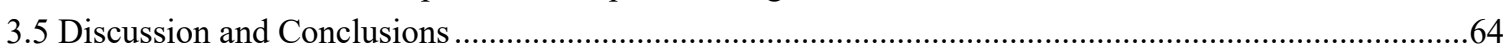

4 Flexible Endoscopy: Optical Molecular Imaging...........................................................67

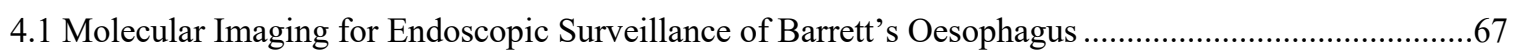

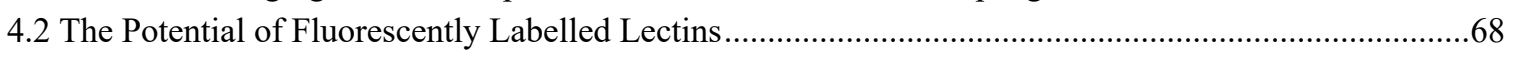

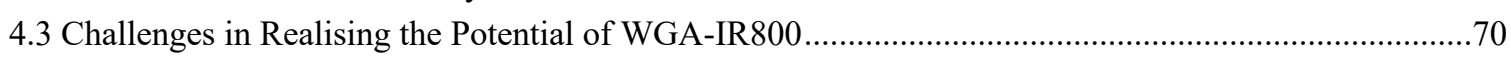

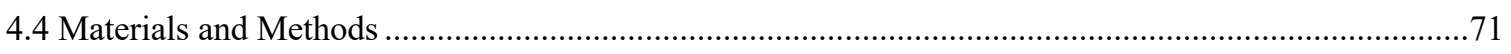

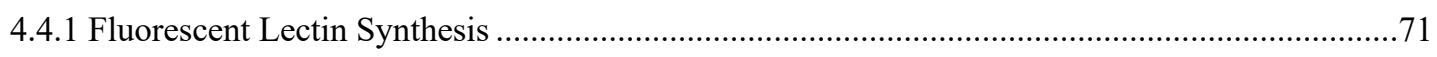

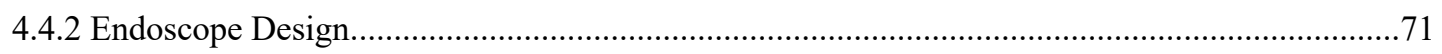




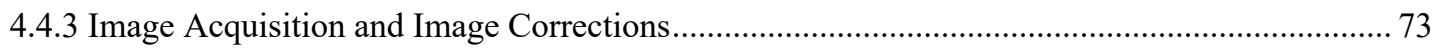

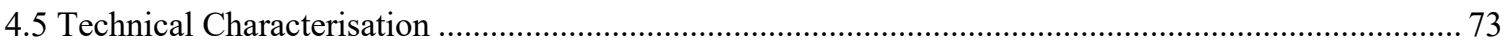

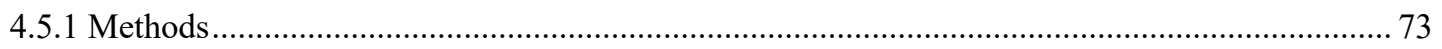

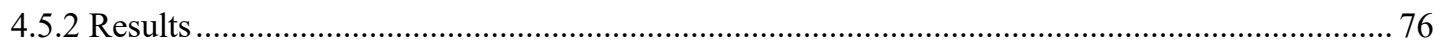

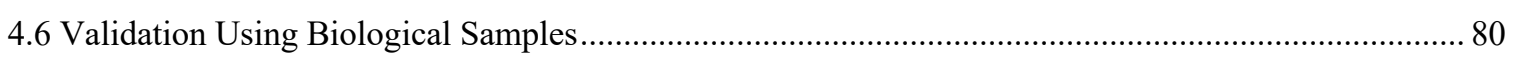

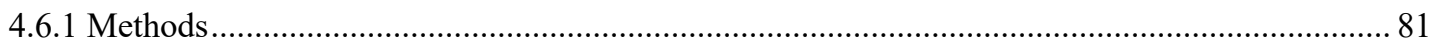

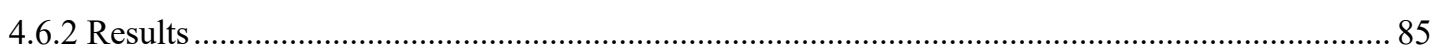

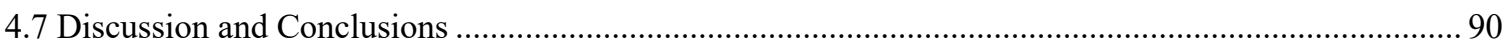

5 Flexible Endoscopy: Multispectral Imaging .......................................................................... 95

5.1 Multispectral Fluorescence Imaging of Targeted Fluorescent Molecules...........................................95

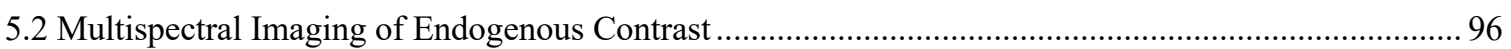

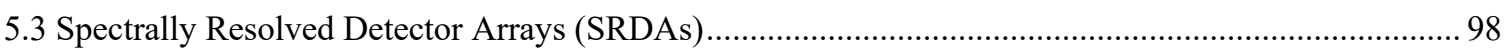

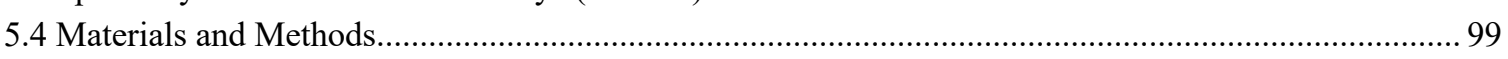

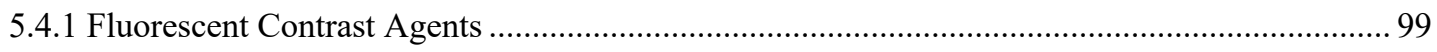

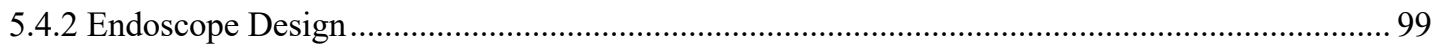

5.4.3 Image Acquisition and Image Corrections...................................................................... 102

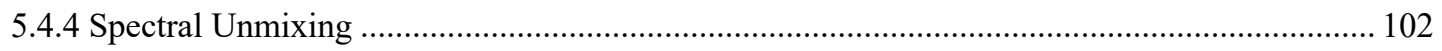

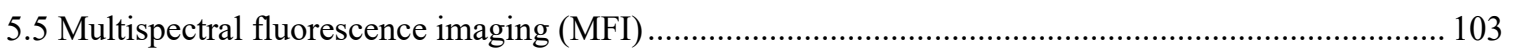

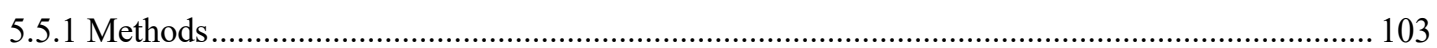

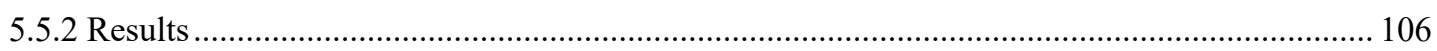

5.6 Multispectral Reflectance Imaging of Endogenous Tissue Contrast................................................. 112

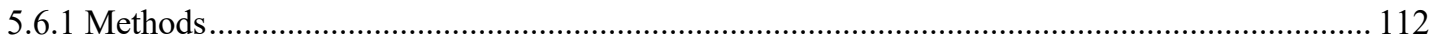

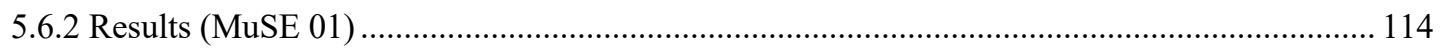

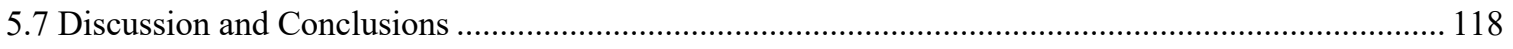

6 Rigid Endoscopy for Intraoperative Imaging of Pituitary Adenoma............................ 123

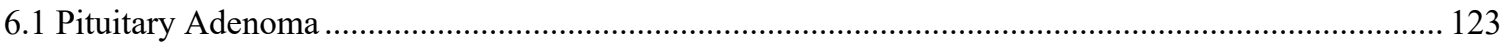

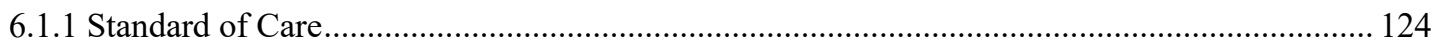

6.1.2 Advanced Imaging of Pituitary Adenoma....................................................................... 124

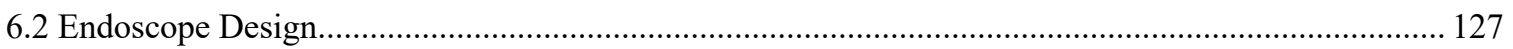

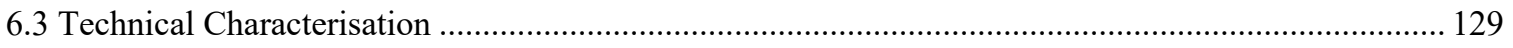

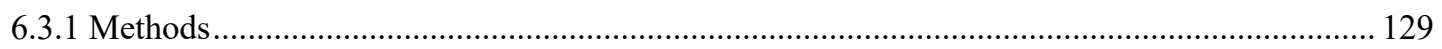

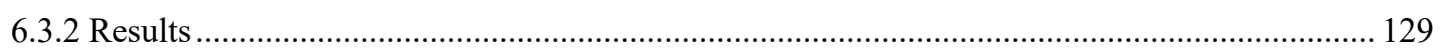

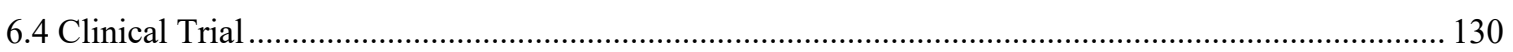

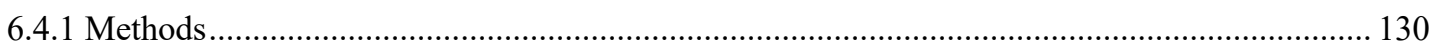

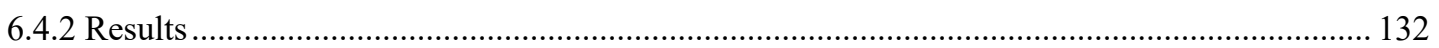

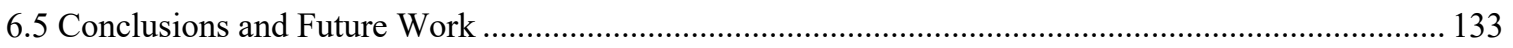

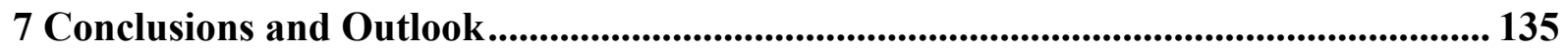

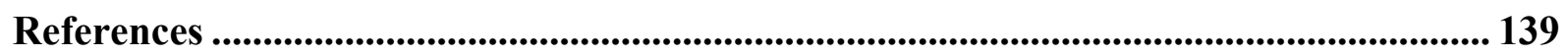

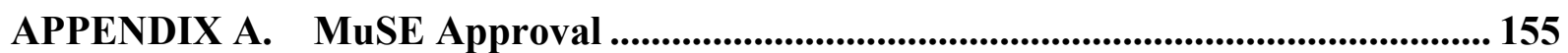

APPENDIX B. MAPS Approval............................................................................................ 162 


\section{List of Publications}

\section{Publications}

Dale J. Waterhouse, James Tysome and Sarah E. Bohndiek, Multispectral Imaging for Delineation of Pituitary Adenomas in Transphenoidal Endoscopy. In preparation.

Jonghee Yoon, James Joseph, Dale J. Waterhouse, A. Siri Luthman, George S. D. Gordon and Massimiliano di Pietro, Wladyslaw Januszewicz, Rebecca C. Fitzgerald, Sarah E. Bohndiek, $A$ clinically translatable hyperspectral endoscopy (HySE) system for imaging the gastrointestinal tract. Accepted in Nature Communications.

Dale J. Waterhouse, Catherine R. M. Fitzpatrick, Brian M. Pogue, James O'Connor and Sarah E. Bohndiek, A roadmap for the clinical implementation of optical imaging biomarkers. Accepted in Nature Biomedical Engineering.

Dale J. Waterhouse, A. Siri Luthman, Jonghee Yoon, George S. D. Gordon and Sarah E. Bohndiek, Quantitative evaluation of comb-structure removal methods for multispectral fiberscopic imaging. Scientific Reports, 8, 17801, (2018).

A. Siri Luthman, Dale J. Waterhouse, Laura Ansel-Bollepalli, Jonghee Yoon, George S. D. Gordon, James Joseph, Massimiliano di Pietro, Wladyslaw Januszewicz and Sarah E. Bohndiek, Bimodal Reflectance and Fluorescence Multispectral Endoscopy based on Spectrally Resolving Detector Arrays. Journal of Biomedical Optics, 24(3), (2018).

Dale J. Waterhouse, Catherine R. M. Fitzpatrick, Massimiliano di Pietro and Sarah E. Bohndiek, Emerging Optical Methods for Endoscopic Barrett's Surveillance. The Lancet Gastroenterology and Hepatology, 3(5), (2018). 
André A. Neves, Massimiliano di Pietro, Maria O’Donovan, Dale J. Waterhouse, Sarah E. Bohndiek, Kevin M. Brindle and Rebecca C. Fitzgerald, Detection of early neoplasia in Barrett's esophagus using lectin-based near-infrared imaging: an ex vivo study on human tissue. Endoscopy, 50(6), (2018).

Dale J. Waterhouse, James Joseph, André A. Neves, Massimiliano di Pietro, Kevin M. Brindle, Rebecca C. Fitzgerald and Sarah E. Bohndiek, Design and validation of a near-infrared fluorescence endoscope for detection of early esophageal malignancy. Journal of Biomedical Optics, 21(8), (2016).

\section{Conference Proceedings}

A. Siri Luthman, Dale J. Waterhouse, Laura Bollepalli, James Joseph and Sarah E. Bohndiek, A multispectral endoscope based on spectrally resolved detector arrays. Proc. SPIE 10411, Clinical and Preclinical Optical Diagnostics, 104110A (2017).

Dale J. Waterhouse, A. Siri Luthman and Sarah E. Bohndiek, Spectral band optimization for multispectral fluorescence imaging. SPIE BiOS (2017).

Massimiliano di Pietro, André A. Neves, Maria O’Donovan, Dale J. Waterhouse, Sarah E. Bohndiek, Kevin M. Brindle and Rebecca C. Fitzgerald, Detection of dysplasia in Barrett's oesophagus using lectin-based near infra-red molecular imaging: an ex-vivo study on human tissue. Proceedings of the British Society of Gastroenterology Meeting (2016).

Dale J. Waterhouse, James Joseph, André A. Neves, Massimiliano di Pietro, Kevin M. Brindle, Rebecca C. Fitzgerald and Sarah E. Bohndiek, Design and validation of a near-infrared fluorescence endoscope for detection of early esophageal malignancy using a targeted imaging probe. SPIE BiOS (2016). 


\section{LIST OF FIGURES}

1.1 Visible and near-infrared light-tissue interactions. 3

1.2 Imaging technique definitions. 3

1.3 Optical Imaging Biomarker Roadmap. 6

$\begin{array}{lll}\text { 2.1 } & \text { Progression of Barrett's oesophagus. } & 17\end{array}$

2.2 Current standard of care for endoscopic surveillance of patients with 17 Barrett's oesophagus.

2.3 'Red flag', 'optical biopsy' and 'hybrid' techniques in relation to the 19 standard of care.

2.4 Advanced optical techniques in clinical use for endoscopic surveillance of 21 Barrett's oesophagus - example images.

2.5 Emerging optical techniques for endoscopic surveillance of Barrett's 27 oesophagus - example images/spectra.

3.1 Endoscopic device architectures - schematics. 37

3.2 The PolyScope accessory channel endoscope. 41

3.3 Advantages and Challenges of the PolyScope disposable endoscope. 41

3.4 Example image with visible comb artefact and mosaic. 43

3.5 Schematic of spectrally resolved detector arrays (SRDAs) and colour filter 43 arrays (CFAs).

3.6 Schematic of the fibrescope. 45

3.7 Schematic of the image simulation process. 45

3.8 Schematic of multispectral demosaicking and comb removal algorithms. 50

3.9 Performance scores for 4 correction methods applied to simulated 56 monochrome images for $\mathrm{M}=0.7, \mathrm{M}=1.0$ and $\mathrm{M}=1.3$.

3.10 Optimum correction method based on overall performance score for 57 simulated images with $\mathrm{M}=0.7, \mathrm{M}=1.0$ and $\mathrm{M}=1.3$.

3.11 Performance scores for 5 correction methods applied to monochrome 58 images.

3.12 Example corrected monochrome images. 59

3.13 Performance scores for 5 correction methods applied to multispectral images 61 for multispectral images.

3.14 Optimum correction method based on overall performance score. 62

3.15 Example corrected multispectral images. 63

4.1 Optical molecular imaging with wheat germ agglutinin. 69

4.2 Schematic of the PolyScope based bimodal NIR fluorescence and WL 72 reflectance endoscope.

4.3 Characterisation of the field of view (FOV) of the bimodal endoscope. 77

4.4 Characterisation of the resolution of the bimodal endoscope. 77 
4.5 Characterisation of the sensitivity of the bimodal endoscope for detection of

NIR fluorescence from WGA-IR800.

4.6 Coregistration of NIR fluorescence images of WGA-IR800 binding to human EMRs.

4.7 WGA-IR800 binding to excised mouse stomach. 86

4.8 NIR fluorescence intensity correlates with dysplasia in punch biopsies. 87

4.9 NIR fluorescence of WGA-IR800 binding to human EMRs captured with the 88 bimodal endoscope and the gold standard imaging device.

4.10 Example co-registration with pathology from EMR B. 89

5.1 SRDA-based endoscope compared to bimodal NIR fluorescence and WL 100 reflectance endoscope.

5.2 SRDA-based multispectral endoscope. 101

5.3 Spectra of the components and contrast agents used for multispectral 102 imaging.

5.4 Spectral response characterisation equipment. 105

5.5 Fluorescence detection characterisation equipment. 105

5.6 Procedure for preparing ex vivo porcine oesophagus for endoscopic imaging. 106

5.7 The spectral response of the detection arm of the SRDA-based multispectral 107 endoscope.

5.8 AF647 abundance measured using the multispectral endoscope and 'ground 109 truth' AF647 abundance measured using a spectrometer.

5.9 Correlation of AF647 abundance measured using the multispectral endoscope with 'ground truth' AF647 abundance measured using a spectrometer.

5.10 Multispectral endoscopic detection of 2 fluorescent dyes (AF647 and AF700) in endoscopy of a whole ex vivo porcine oesophagus.

5.11 Procedure for in vivo clinical trial of multispectral endoscopy (MuSE).

5.12 EMR specimen from MuSE 01.

5.13 Synchronised HD-WLE and multispectral images from the first-in-human 116 trial of the multispectral endoscope (MuSE 01).

5.14 Spectra of Barrett's oesophagus and intramucosal carcinoma measured with 117 the multispectral endoscope.

6.1 Simple schematic showing transphenoidal surgery for pituitary adenoma. $\quad 125$

6.2 Schematic of the SRDA-based rigid multispectral endoscope. 128

6.3 Characterisation of the resolution of the rigid multispectral endoscope. 131

6.4 Characterisation of the FOV of the rigid multispectral endoscope. 131 


\section{LIST OF TABLES}

1.1 Optical endoscopic imaging techniques in development in oncology.

2.1 Advanced optical techniques in clinical use for endoscopic surveillance of 20 Barrett's oesophagus.

2.2 Emerging optical techniques for endoscopic surveillance of Barrett's 26 oesophagus.

3.1 Endoscopic device architectures. 36

4.1 Optical molecular imaging techniques in trials for surveillance of Barrett's 68 oesophagus.

4.2 Experiments used to validate WGA-IR800 imaging with the bimodal NIR 80 fluorescence and WL reflectance endoscope. 


\section{LIST OF APPENDICES}

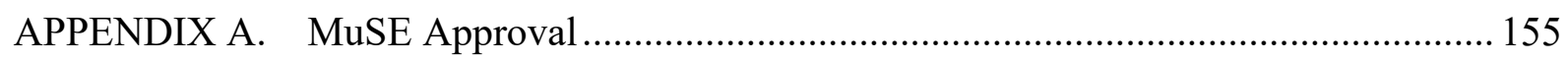

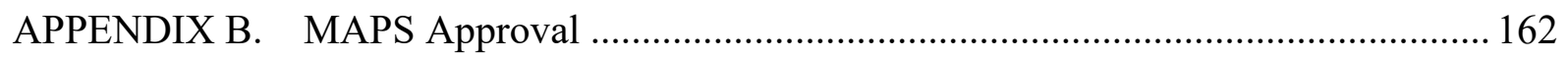




\section{LIST OF ABBREVIATIONS}

4QB four quadrant biopsies according to the Seattle protocol

a/LCI angle-resolved low coherence interferometry

AA acetic acid

ACG American College of Gastroenterology

AF647/700 AlexaFluor 647/700

AFI autofluorescence imaging

AGA American Gastroenterology Association

ASGE American Society for Gastrointestinal Endoscopy

ASR accuracy of spectral reconstruction

AUC area under the curve

BLI blue laser imaging

BSG British Society of Gastroenterologists

CARS coherent anti-Raman spectroscopy

CCD charge coupled device

CFA colour filter array

CMOS complementary metal-oxide-semiconductor

CT computed tomography

DRS diffuse reflection spectroscopy

eCLE endoscope-based confocal laser endomicroscopy

EM electron multiplying

EMCCD electron multiplying charge coupled device

EMR endoscopic mucosal resection

ERCP endoscopic retrograde cholangiopancreatography

ESGE European Society for Gastrointestinal Endoscopy

ESS elastic scattering spectroscopy

ETMI endoscopic trimodal imaging

FDA food and drug administration

FICE Fujicon intelligent chromoendoscopy

FLIM fluorescence lifetime imaging

FOV field of view

FWHM full width half maximum

GI gastrointestinal

GMP good manufacturing practice 


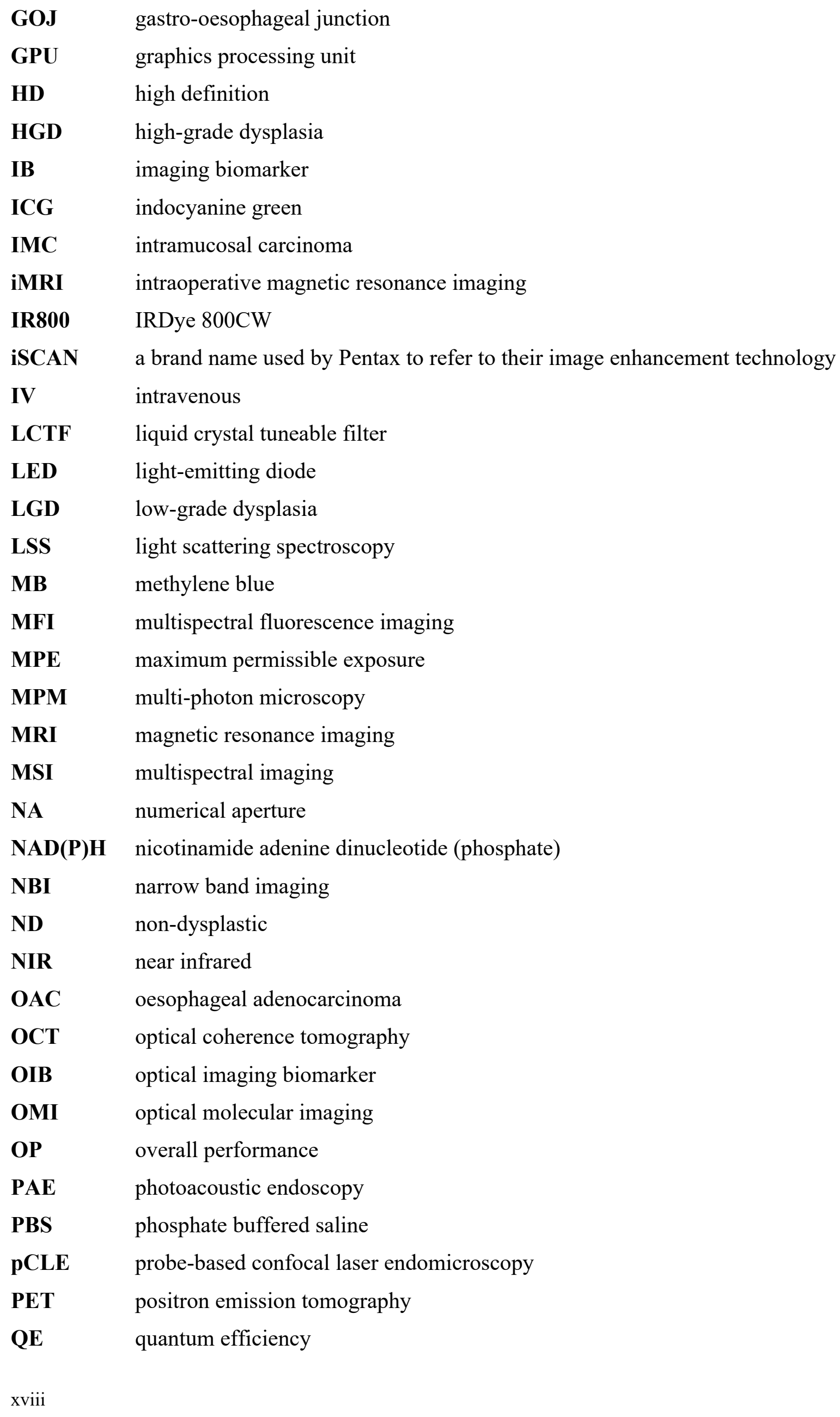




$\begin{array}{ll}\text { RGB } & \text { red green blue } \\ \text { ROI } & \text { region of interest } \\ \text { RS } & \text { Raman spectroscopy } \\ \text { SBR } & \text { signal to background ratio } \\ \text { SNR } & \text { signal to noise ratio } \\ \text { SOP } & \text { standard operating procedure } \\ \text { SRDA } & \text { spectrally resolved detector array } \\ \text { TC } & \text { translational characteristic } \\ \text { UHD } & \text { ultra-high definition } \\ \text { UHP } & \text { ultra-high powered } \\ \text { UTNE } & \text { un-sedated trans-nasal endoscopy } \\ \text { VI } & \text { visual interface } \\ \text { VLE } & \text { volumetric laser endomicroscopy } \\ \text { WD } & \text { working distance } \\ \text { WGA } & \text { wheat germ agglutinin } \\ \text { WL } & \text { white light } \\ \text { WLE } & \text { white light endoscopy/endoscope }\end{array}$





\section{TRANSLATION OF OPTICAL IMAGING TECHNIQUES}

This chapter uses material from a perspective paper by the author entitled: "A roadmap for the clinical implementation of optical imaging biomarkers" (see publications list).

Optical imaging has tremendous potential for non-invasive detection and characterisation of diseased tissue using non-ionising radiation with wavelengths from 400-1000 nm. The approach benefits from: real-time analysis of tissue biochemistry based on sensitive interactions of light and tissue; compact, point-of-care and low-cost implementations compared to radiological imaging; and operation across a range of resolutions and depths that span over 4 orders of magnitude [1].

This thesis examines the potential of optical imaging in two key indications: early detection of cancer in the oesophagus and delineation of cancer and healthy tissue in resection of pituitary tumours. Before embarking on the development of novel devices, an analysis was made of the common challenges in translating optical imaging techniques.

\subsection{The Potential of Optical Imaging in Oncology}

Visible and near infrared (NIR) light undergo a wide range of complex interactions in tissue, including absorption, reflection, elastic and inelastic scattering, and fluorescence absorption and emission [2] (Figure 1.1). Conventional imaging discards this rich information, instead capturing reflected red, green and blue light to simply replicate human vision [3]. Instead of discarding this information, we can exploit it to yield optical imaging biomarkers (OIBs), defined characteristics measured as indicators of biology, often derived from measurements 
made on an image (Figure 1.2) [4]. A large number of OIBs have been developed to assess hallmarks of cancer [5], ranging from microstructural change and collagen cross-linking associated with replicative immortality and activating invasion, to haemoglobin concentration and oxygenation, associated with induction of angiogenesis. The ability to use these biomarkers to generate high contrast for cancer, has led to clinical approval of dozens of optical imaging techniques for early cancer detection (Table 1.1).

Despite this potential, and the obvious enthusiasm for such technologies to reach clinical implementation, whilst many techniques have been approved for use in humans, relatively few have reached routine use in healthcare systems, begging the question: what is preventing their translation into healthcare?

\subsection{Challenges of Clinical Translation of Optical Imaging Techniques}

Development of novel optical imaging techniques often begins with the discovery of promising OIBs using optical devices dedicated to ex vivo imaging. This can motivate the development of devices able to measure the same biomarker in vivo (this is our objective in Chapter 4). Alternatively, development may begin when application of a contrast mechanism and/or device architecture found to be useful in another field is applied to a new biological question (this is our objective in Chapter 6).

For any new imaging biomarker to be deployed in healthcare, detailed technical validation is required to define the precision and accuracy with which the biomarker can be measured. Biological validation, establishing the association between the biomarker and the underlying physiological, anatomical, or pathological process, should be performed in parallel with technical validation. Importantly, clinical validation, establishing that the biomarker identifies, measures, or predicts the outcome of interest, can be performed only with an imaging device that is approved for use in patients.

Considering these validation processes, there are two main differences in the clinical translation of a novel OIB compared to an imaging biomarker developed for standard radiological imaging, such as computed tomography (CT) or magnetic resonance imaging (MRI), where the imaging device is already clinically approved for use in humans and widely available in hospital radiology departments [4]. Firstly, for OIBs, it is uncommon that an existing clinically approved imaging device, with associated specialist data acquisition and interpretation methods, will already be available to allow clinical validation of the biomarker. As a result, biological 


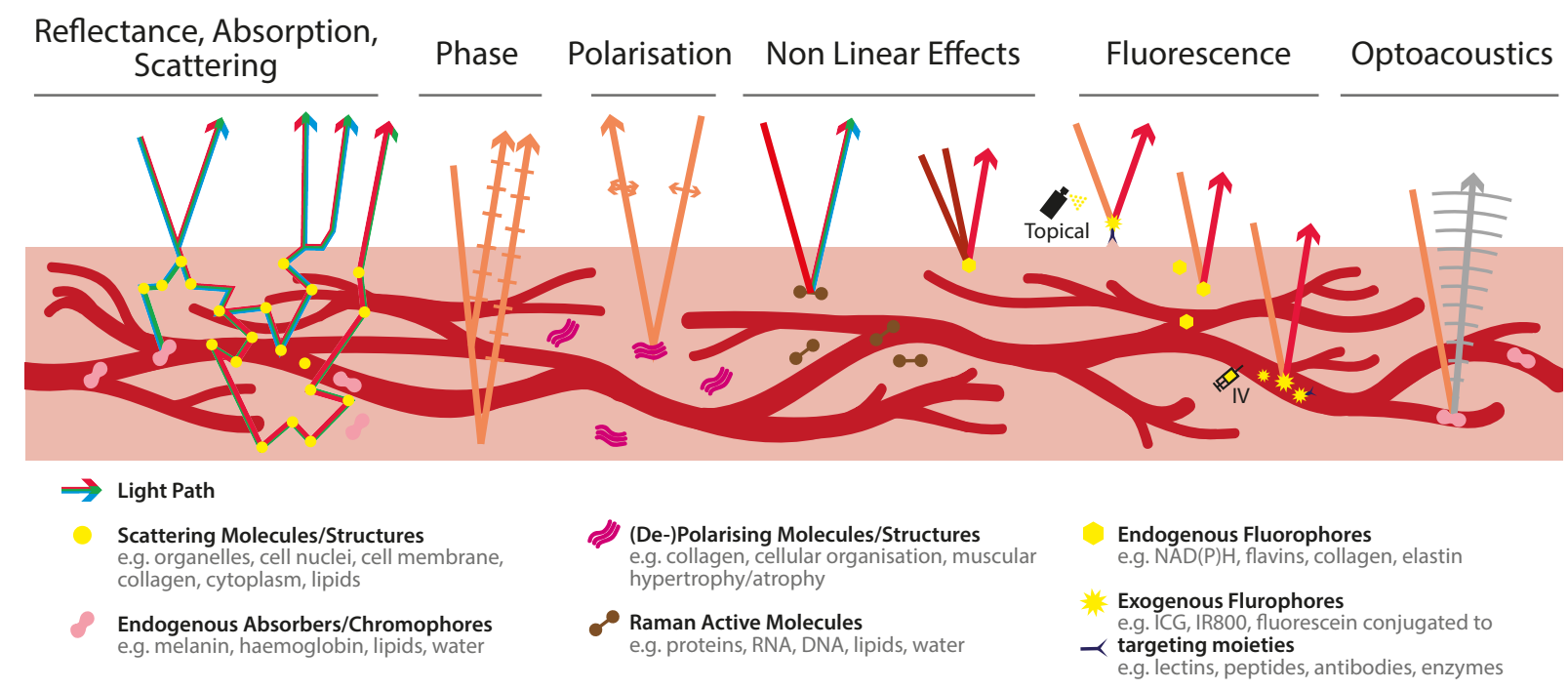

\section{Figure 1.1 Visible and near-infrared light-tissue interactions.}

Visible and near-infrared light undergo a wide range of complex interactions with tissue constituents. Novel optical imaging techniques attempt to use these interactions as a source of contrast for clinical imaging. Interactions illustrated include: reflection, absorption and scattering (e.g. white light imaging, diffuse reflectance spectroscopy, narrow band imaging); phase (e.g. optical coherence tomography); polarisation (e.g. polarimetry); non-linear effects (e.g. Raman spectroscopy, multi-photon fluorescence imaging,); fluorescence (e.g. autofluorescence intensity or lifetime imaging of endogenous fluorophores, optical molecular imaging of exogenous fluorophores); and the optoacoustic effect (e.g. photo (or opto) acoustic microscopy or tomography). Line colours represent colours of light. Multi-coloured lines represent broadband light. Changes of colour upon interaction represent change of wavelength, for example, during fluorescence the change from orange to red signifies shorter wavelength absorption and longer wavelength emission. Perpendicular lines represent wave fronts and thus show optical coherence of phase. Arrowed perpendicular lines represent polarisation orientation. Curved lines represent emission of acoustic waves. (IV=intravenous).

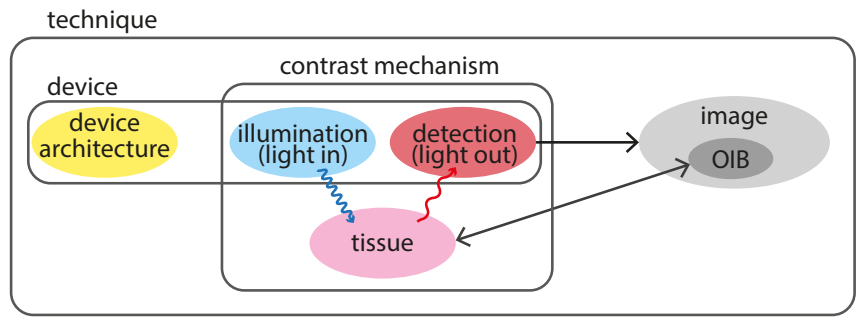

\section{Figure 1.2 Imaging technique definitions.}

The terminology surrounding optical imaging is not standardised in the literature. Throughout this thesis we refer to the illumination or 'light in' (e.g. $300 \mathrm{~nm}$ light), detection or 'light out' (e.g. $400 \mathrm{~nm}$ light), tissue constituent of interest (e.g. collagen) and their light-tissue interaction (e.g. collagen fluorescence) as the contrast mechanism. When the choice of light is combined with a device architecture (e.g. microscope, endoscope), we refer to this as a device. The light out is used to create an image that encodes the underlying light-tissue interaction. Optical imaging biomarkers (OIBs) are defined characteristics of the image. These could be simple, such as image intensity, or more complex, such as degree of branching in vascular pattern. Once properly validated, OIBs can act as indicators of the underlying tissue biology, for example disease pathology. We refer to the combination of device, contrast mechanism, and OIB as a 'technique'. We avoid use of the term 'modality' as it is used variably throughout the literature. Note that these definitions may be different to those used in non-optical imaging. 
Table 1.1 Optical endoscopic imaging techniques in development in oncology.

FOV: field of view.

\begin{tabular}{|c|c|c|c|c|}
\hline Approach & Source of contrast & $\begin{array}{l}\text { Biological change } \\
\text { in cancer }\end{array}$ & Example OIB & $\begin{array}{l}\text { Clinically approved } \\
\text { commercial devices }\end{array}$ \\
\hline $\begin{array}{l}\text { White Light } \\
\text { Imaging [6] }\end{array}$ & \multirow{2}{*}{$\begin{array}{l}\text { Endogenous chromophores (e.g. } \\
\text { melanin, haemoglobin, } \\
\text { tryptophan, lipids, water); } \\
\text { endogenous scatterers (e.g. } \\
\text { mitochondria, cell nuclei, cell } \\
\text { membrane) }\end{array}$} & \multirow[b]{2}{*}{$\begin{array}{l}\text { Vasculature, } \\
\text { melanin } \\
\text { distribution, visible } \\
\text { lesions }\end{array}$} & $\begin{array}{l}\text { Mucosal } \\
\text { abnormalities }\end{array}$ & $\begin{array}{l}\text { Widely available endoscopes and } \\
\text { dermoscopes, naked eye } \\
\text { examination }\end{array}$ \\
\hline $\begin{array}{l}\text { Virtual (or } \\
\text { Electronic) } \\
\text { Chromoendoscopy } \\
{[7-11]}\end{array}$ & & & $\begin{array}{l}\text { Irregular } \\
\text { mucosal and } \\
\text { vascular patterns }\end{array}$ & $\begin{array}{l}\text { Olympus NBI Scopes [12], } \\
\text { Fujicon FICE [13]. PENTAX } \\
\text { iScan [14], SIMSYS- } \\
\text { MoleMate }{ }^{\mathrm{TM}}[15], \text { MelaFind } ® \\
\text { [16], Spectral Molecular Imaging } \\
\text { SkinSpect }^{\mathrm{TM}} \text { [17] }\end{array}$ \\
\hline Polarimetry [18] & Microstructural anisotropy & $\begin{array}{l}\text { Collagen, cellular } \\
\text { orientations, } \\
\text { organisation, } \\
\text { muscular } \\
\text { hypertrophy/atrophy }\end{array}$ & Unclear & Derma Medical MoleMax 3 [19] \\
\hline \multicolumn{5}{|l|}{ Dye-based Imaging } \\
\hline $\begin{array}{l}\text { Chromoendoscopy } \\
{[9,11]}\end{array}$ & $\begin{array}{l}\text { Dyes/Stains (e.g. methylene } \\
\text { blue, acetic acid). Can enhance } \\
\text { surface topology or be } \\
\text { differentially absorbed by } \\
\text { different cell types }\end{array}$ & $\begin{array}{l}\text { Visible lesions, cell } \\
\text { type }\end{array}$ & $\begin{array}{l}\text { Loss of } \\
\text { acetowhitening }\end{array}$ & Widely available endoscopes \\
\hline $\begin{array}{l}\text { Targeted } \\
\text { Fluorescence } \\
\text { Imaging }[20,21]\end{array}$ & $\begin{array}{l}\text { Exogenous fluorophores } \\
\text { conjugated to targeting moieties } \\
\text { (lectins, peptides, antibodies, } \\
\text { affibodies, enzymes) that target } \\
\text { intracellular and extracellular } \\
\text { proteins and enzymes }\end{array}$ & $\begin{array}{l}\text { Changes in targeted } \\
\text { intracellular and } \\
\text { extracellular } \\
\text { proteins and } \\
\text { enzymes }\end{array}$ & $\begin{array}{l}\text { High intensity } \\
\text { fluorescence due } \\
\text { to uptake or } \\
\text { binding of probe }\end{array}$ & None \\
\hline $\begin{array}{l}\text { Endoscopic } \\
\text { Microscopy }[9,22]\end{array}$ & $\begin{array}{l}\text { Exogenous fluorophores (e.g. } \\
\text { fluorescein) }\end{array}$ & Cell type & $\begin{array}{l}\text { Change in cell } \\
\text { phenotype such } \\
\text { as irregular } \\
\text { borders and } \\
\text { shapes }\end{array}$ & $\begin{array}{l}\text { Mauna Kea Cellvizio }{ }^{\circledR}[23] \text {, } \\
\text { Optiscan ViewnVivo }{ }^{\mathrm{TM}}[24] \text {, } \\
\text { Caliber ID VivaScope }[25]\end{array}$ \\
\hline \multicolumn{5}{|c|}{ Autofluorescence Imaging } \\
\hline $\begin{array}{l}\text { Autofluorescence } \\
\text { Intensity Imaging } \\
{[26]}\end{array}$ & \multirow{3}{*}{$\begin{array}{l}\text { Endogenous fluorophores (e.g. } \\
\text { NAD }(\mathrm{P}) \mathrm{H} \text {, flavins, collagen, } \\
\text { elastin, phenylalanine, } \\
\text { tryptophan) }\end{array}$} & \multirow{3}{*}{$\begin{array}{l}\text { Biochemical e.g. } \\
\text { collagen cross-link } \\
\text { breaking }\end{array}$} & $\begin{array}{l}\text { Loss of } \\
\text { autofluorescence, } \\
\text { denoted by } \\
\text { purple image } \\
\text { highlight }\end{array}$ & Widely available endoscopes \\
\hline $\begin{array}{l}\text { Fluorescence } \\
\text { Lifetime Imaging } \\
{[27-29]}\end{array}$ & & & $\begin{array}{l}\text { Change in } \\
\text { melanocyte } \\
\text { distribution }\end{array}$ & JenLab DermaInspect [30] \\
\hline $\begin{array}{l}\text { Multi Photon } \\
\text { Microscopy [27- } \\
\text { 29] }\end{array}$ & & & $\begin{array}{l}\text { Change in } \\
\text { melanocyte } \\
\text { distribution }\end{array}$ & JenLab DermaInspect [30] \\
\hline \multicolumn{5}{|c|}{ Cross Sectional Imaging } \\
\hline $\begin{array}{l}\text { Optical Coherence } \\
\text { Tomography [31] }\end{array}$ & $\begin{array}{l}\text { Boundaries between structural } \\
\text { features }\end{array}$ & \multirow{2}{*}{$\begin{array}{l}\text { Vasculature, visible } \\
\text { lesions, structural } \\
\text { changes }\end{array}$} & $\begin{array}{l}\text { Change in } \\
\text { structure such as } \\
\text { irregular } \\
\text { glandular } \\
\text { architecture }\end{array}$ & $\begin{array}{l}\text { Ninepoint Medical } \\
\text { NvisionVLE® [32], Verisante } \\
\text { Core }^{\mathrm{TM}} \text { [33], Skintell [34], } \\
\text { Michelson Diagnostics Vivosigh } \\
\text { [35] }\end{array}$ \\
\hline $\begin{array}{l}\text { Photoacoustic } \\
\text { Imaging }\end{array}$ & $\begin{array}{l}\text { Absorption of endogenous } \\
\text { chromophores (e.g. melanin, } \\
\text { haemoglobin) }\end{array}$ & & $\begin{array}{l}\text { Reduction in } \\
\text { oxygen } \\
\text { saturation }\end{array}$ & None \\
\hline \multicolumn{5}{|l|}{ Spectroscopy } \\
\hline $\begin{array}{l}\text { Raman } \\
\text { Spectroscopy [36- } \\
\text { 39] }\end{array}$ & $\begin{array}{l}\text { Vibrational modes of specific } \\
\text { molecules (e.g. proteins, DNA, } \\
\text { lipids) }\end{array}$ & Biochemical & $\begin{array}{l}\text { Change in } \\
\text { spectrum }\end{array}$ & Verisante Aura $^{\mathrm{TM}}[40]$ \\
\hline $\begin{array}{l}\text { Reflection } \\
\text { Spectroscopy }[41, \\
42]\end{array}$ & $\begin{array}{l}\text { Reflection spectra of } \\
\text { endogenous biological } \\
\text { molecules }\end{array}$ & Biochemical & $\begin{array}{l}\text { Change in } \\
\text { spectrum }\end{array}$ & Pentax WavSTAT4 ${ }^{\mathrm{TM}}[14]$ \\
\hline $\begin{array}{l}\text { Diffuse } \\
\text { Reflectance } \\
\text { Spectroscopy }[43, \\
44]\end{array}$ & $\begin{array}{l}\text { Endogenous scatterers (e.g. } \\
\text { mitochondria, cell nuclei, cell } \\
\text { membrane, collagen, cytoplasm, } \\
\text { lipids) }\end{array}$ & $\begin{array}{l}\text { Vasculature, change } \\
\text { in distribution of } \\
\text { cells, nuclei and } \\
\text { organelles }\end{array}$ & $\begin{array}{l}\text { Change in } \\
\text { spectrum }\end{array}$ & None \\
\hline
\end{tabular}


validation may be restricted to testing with ex vivo samples, such as histopathological sections, which can be prone to bias and generate a different range of optical interactions compared to the in vivo setting; the result can be misleading conclusions as to the potential clinical utility of the OIB $[9,45]$.

A second key difference between optical and radiological imaging biomarkers is that OIBs may be deployed in a range of settings in the clinical patient management pathway, spanning the home; primary care (family physician); and specialist care (specialist practice, referral or medical centre). Thus, even a well-defined biomarker with promising performance in the experimental setting may never receive approval if it fails to adequately address a specific diagnostic question at the appropriate point in the care pathway.

\subsection{The Optical Imaging Biomarker Roadmap}

With the differences between radiological imaging biomarkers (IBs) and OIBs in mind, we developed a specific 'Optical Imaging Biomarker Roadmap' (Figure 1.3) based on the international consensus 'Imaging Biomarker Roadmap' created for use in cancer studies [4]. The aim of the roadmap is to elucidate the reasons for failure in clinical translation of optical imaging techniques and to overcome these limitations to allow smoother translation of promising techniques into clinic.

In all domains, optical device development plays a larger role in translation than in standard radiological imaging, where a device to measure the imaging biomarker is usually already clinically approved. Throughout the OIB Roadmap, there is a complex interplay of limitations imposed by the device, the contrast mechanism and the OIB, meaning technical and biological validation cannot be considered in isolation. For example, the precision (repeatability / reproducibility) of measuring a given OIB defined with respect to a perfect test target will differ considerably from precision defined with respect to the biological measurement made in a patient, which is directly relevant in the clinical application. Common feedback loops that often inhibit translation are highlighted so that advance consideration might allow developers to avoid them. Construction of the roadmap revealed five key areas where experimental methodologies or regulations can create translational barriers. These will be referred to throughout this thesis, emphasizing where their consideration has influenced the methodologies adopted, and outlining the part of the roadmap our work intends to address. 


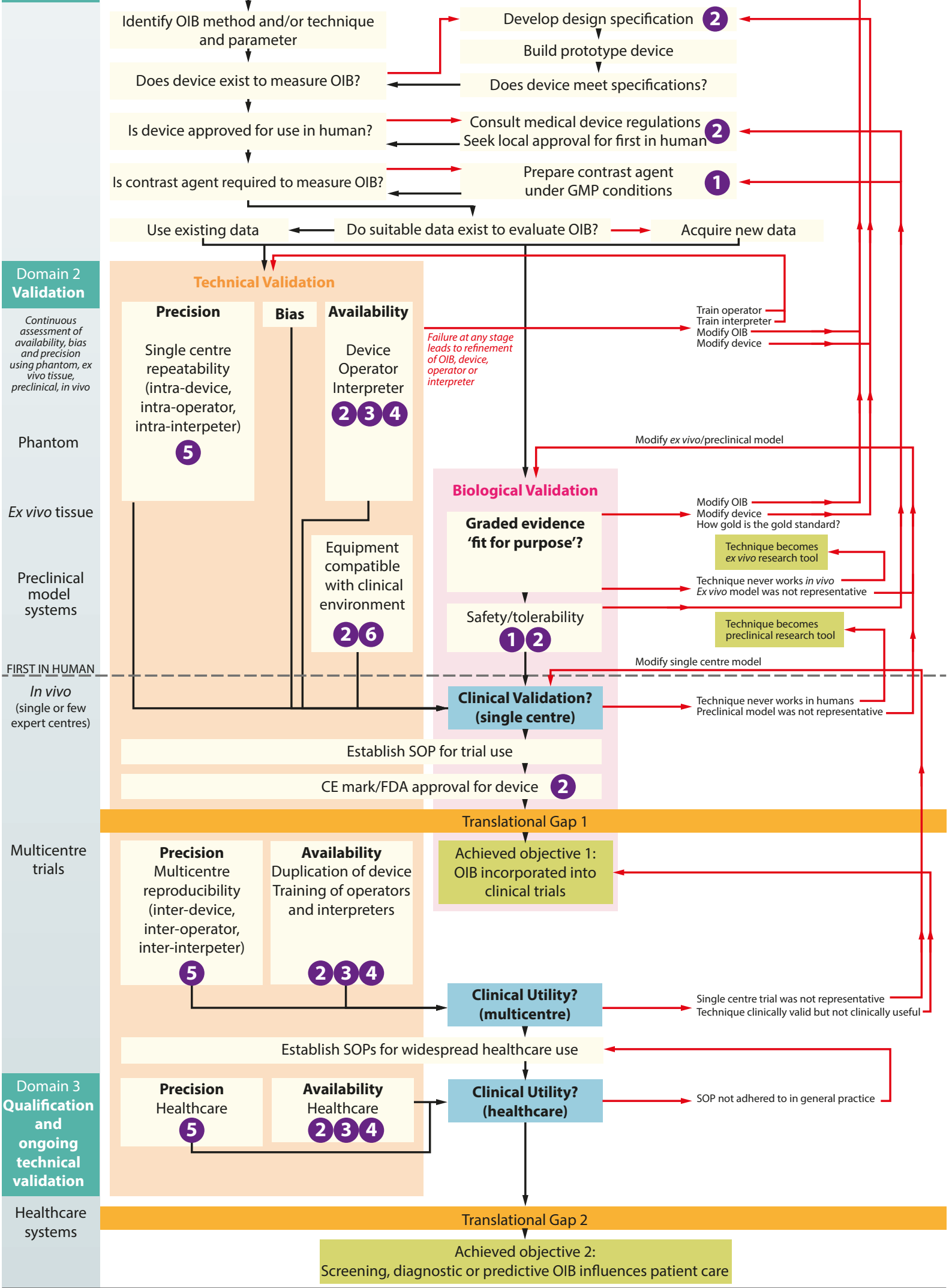

Translational Characteristics

(1) Contrast Mechanism 


\section{Figure 1.3 Optical Imaging Biomarker Roadmap.}

This proposed optical imaging biomarker roadmap differs from the imaging biomarker roadmap [4] by considering the intrinsic coupling of clinical translation to the development of the optical imaging device. Red feedback loops decelerate clinical translation. Purple circles represent points at which careful advance consideration of translational characteristics 1-6 can accelerate translation (Section 1.3.2). Translational characteristics are: 1. Contrast Mechanism. 2. Instrumentation. 3. Operator Expertise. 4. Image Reader Expertise 5. Repeatability and Reproducibility 6. Co-registration. In the optical imaging biomarker roadmap, the technical validation of the technique is more prominent than in the imaging biomarker roadmap, since each new optical imaging technique requires the development of a partially or entirely new optical device. Technical and biological validation occur in parallel and are closely linked due to the interplay between device, operator, interpreter and optical imaging biomarker. Essential technical validation occurs throughout the roadmap to ensure availability and precision in all settings from phantoms to healthcare. Cost-effectiveness is omitted here but impacts on the roadmap at every stage, owing to the equipment and personnel costs of performing imaging studies [4]. SOP: standard operating procedure, OIB: optical imaging biomarker.

\subsubsection{Barriers to Translation of Optical Imaging Techniques}

\section{Translational Barrier 1. Lack of defined safe exposure limits for diagnostics}

Visible and near-infrared light is not ionising, ( $\mathrm{H}$ ionisation occurs at $\sim 120 \mathrm{~nm}$ ), but can lead to thermal damage, photosensitisation and photoallergic reactions under certain conditions [46]. Molecularly damaging radiation extends further towards the visible spectrum [47], so safe and effective illumination levels, those that allow accurate measurement of a given biomarker, at a safe optical radiation level, must be established before in vivo trials take place. The published guidelines for optical radiation exposure limits only concern the retina and skin, and are limited in their application to workers rather than patients, which presents a challenge for those using incoherent light and internal diagnostic imaging devices [46, 48]. There is an unmet need for experimentally validated exposure limits for other tissues so that safe patient exposures for optical imaging can be clearly defined. This would help to address frequently raised concerns of clinical trials ethical review boards. Furthermore, the availability of optical radiation protection advisors with training in clinical optical imaging to advise ethical review boards would be advantageous, to increase understanding of the capabilities, strengths and weaknesses of optical imaging systems and their biomarkers.

\section{Translational Barrier 2. Lack of standardised quality assurance}

The uncertainty regarding optical exposure limits is illustrative of a more general lack of standardisation in optical imaging. Defining the performance of a device at the point of manufacture and throughout lifetime is crucial to enable comparison of different devices measuring the same source of optical contrast and debug device-specific problems. 
Whilst performance standards (documents that suggest relevant performance characteristics and test methods) exist for the established non-optical imaging methods, such as the National Electrical Manufacturers Association (NEMA) imaging standards for positron emission tomography (PET) [49], optical imaging is currently without published standards [50]. As a result, optical imaging contrast agents and devices must be often approved in tandem. This is in contrast to PET where contrast agents are usually approved for use in a range of PET devices, and vice versa, due to sufficient evidence supporting the similarity across PET devices [50]. Unfortunately, the consequence for optical imaging is that healthcare providers must purchase specific devices when working with specific optical imaging agents, resulting in prohibitive costs and blocking translation of new agents.

In the US and Europe, medical devices may be approved based on similarity to a predicate device, gaining $510(\mathrm{k})$ clearance in the US and CE marking in the EU [51,52]. But without proper standardisation, similarity to a predicate may be difficult to define, leaving performance unclear. Even worse, the predicate could predate the introduction of regulatory standards. With proper imaging standards, device performance could be properly compared, allowing inferiority to be spotted at an earlier stage in the translational roadmap. Additionally, if device performance standards were established it would help separate device approval from agent approval, in cases where exogenous agents are being imaged.

\section{Translational Barrier 3. Lack of accurate, validated, clinical gold standards}

New techniques are often compared to clinical gold standards to measure clinical performance, but these are a common source of problems limiting translation. Most commonly, gold standard diagnosis is determined by assessment of stained tissue sections by a pathologist. To achieve the most accurate gold standard for translation of optical imaging biomarkers, consensus of several independent pathologists is needed, as they are not always in agreement [53]. In the best-case scenario, only unanimous decisions would be accepted, though this is difficult to achieve in practice as it significantly decreases the number of samples that can be incorporated into any calculation. In the future, machine learning algorithms trained on huge datasets may be able to provide an objective diagnosis, but this is still some way from being realised.

Another confounding factor is the transition from a biopsy read to in vivo imaging, which requires an appreciation of the nuances of a field of tissue being a mix of pathology as well as normal anatomy. Each optical imaging pixel could include contributions from one or more tissue states, whereas a histopathology analysis will typically report only the highest grade present. In addition, high congruence in spatial alignment between the in vivo and ex vivo coordinates is required. Once the gold standard is validated, careful thought must be applied to how these findings will be accurately co-registered with optical images, so as not to introduce 
further artefacts into the comparison between the novel technique and the gold standard. The different scales at which in vivo optical image data is recorded and histopathological analysis is performed can make this particularly challenging [54]. Throughout this thesis, where novel techniques are compared to gold standards, methods were carefully designed to simplify coregistration.

\section{Translational Barrier 4. Lack of validated, representative ex vivo models}

To avoid disappointing in vivo results later in the roadmap, it is also crucial to ensure that the ex vivo model is as representative as possible of the in vivo situation. When tissue is excised from the body, several OIBs change irreversibly. For example, the lack of active blood flow changes the spectrum of tissue by reducing blood oxygenation to $0 \%$ and consequently altering the haemoglobin absorption spectrum. Furthermore, tissue autofluorescence can be modified upon exposure to ambient light and tissue structure may be distorted by surgical trauma, or by positioning the tissue on a rigid surface. Ultimately, tissue will degrade unless fixed in formaldehyde or frozen, which further alter properties [55]. The leap between data acquired ex vivo and in vivo imaging is therefore large and data acquired from ex vivo tissues may contain insurmountable artefacts if the tissues are not properly handled.

These challenges with using ex vivo tissue for validation raise the need for new model systems, which may arise through improved tissue mimicking phantoms, or from bioengineering of artificial tissues. Whichever route is chosen, to avoid disappointing in vivo results later in the roadmap, it is crucial to ensure that the ex vivo model is as representative as possible of the in vivo situation. For example, modalities that are sensitive to blood oxygenation should never use excised tissue for validation unless it is perfused, while modalities sensitive to preparation artefacts should avoid chemically fixed or frozen tissue [55] and dyes that incorporate labelled human antibodies should use models that try to replicate human biology, such as human derived tumour xenografts, rather than animal tumour models where the binding affinity may be different.

\section{Translational Barrier 5. Difficulty in conducting representative single centre trials}

Similarly, early stage single centre trials should replicate the common clinical environment as far as possible. Firstly, representative populations should be chosen to reduce spectrum effects [56]. For example, due to the ethical considerations of taking biopsies from healthy tissue, many skin imaging trials have been carried out in enriched populations with high disease incidence, which has prompted investigators to endorse the need for a mix of lesions representative of the target population to be used for future testing of new approaches [57] and encouraging regulatory bodies to disregard earlier results. Secondly, standard operating procedures (SOPs) 
should be determined by a single centre and adhered to in multi-centre trials in order to prevent bias. For example, inspecting images for minutes when the eventual SOP in a clinical setting would require inspection of videos in real time gives misleading performance evaluations [5861]. Thirdly, the expertise of the operator and interpreter should be representative of that realistically achieved in routine care. Endoscopic trimodal imaging (Chapter 2.2.2) provides a cautionary example where promising results in a specialist tertiary referral centre [62] were not reproduced in a community practice setting [63], which can be due to a combination of spectrum effects, different SOPs and different expertise.

\subsubsection{Translational Characteristics of Optical Imaging Techniques}

In constructing the OIB Roadmap, we identified several 'translational characteristics' (TCs) that seem to be common among those techniques that have been widely adopted. These characteristics do not define technical performance (such as resolution or signal to noise ratio) or clinical performance (such as negative predictive value or specificity) but rather characterise the amenability of a technique to clinical translation. The OIB Roadmap highlights the key points at which consideration of these translational characteristics can be exploited to accelerate translation (Figure 1.3). The translational characteristics are detailed below together with suggestions for how to best achieve translation.

Translational Characteristic 1. Contrast Mechanism. Exploiting endogenous contrast (imaging without application of dyes) to derive the OIB is favourable in terms of the clinical pathway. Still, exogenous contrast agents can be beneficial for improving the contrast of cancer compared to healthy tissue, both as non-specific stains [64] and targeted molecular imaging agents [21], but they require synthesis at good manufacturing practice (GMP) standards, associated toxicology studies, and in addition to the need for specific instrumentation, also add procedure time and cost $[20,65]$. Several aspects of contrast agent chemistry increase the likelihood of their successful clinical translation: having a validated target (structural or molecular) increases confidence that results will be reproducible; topically rather than intravenously administered agents limit the exposure to the tissue of interest and speed up procedures; and agents with long term stability once formulated are favourable for storage and distribution.

Translational Characteristic 2. Instrumentation. A regulatory body such as the FDA must approve new devices [50, 51, 66, 67]. For clinical implementation, compact, robust and transportable optical imaging devices devoid of complex delicate optics are highly desirable. Devices that are compatible with existing systems, or include current standard of care methods 
for reference, are more likely to be translated, perhaps initially as an adjunct to an existing technique, which also facilitates head-to-head trials.

Translational Characteristic 3. Operator Expertise. The potential operators of a new optical imaging technique range from the untutored public through professionals working in primary care to highly specialised individuals working in a specialist care centre. A new approach must either: give similar clinical results to an existing approach, but with less expertise required; reduce the required expertise sufficiently to allow translation of the approach from expert to generalist setting, reducing the burden on specialist care centres and reducing the cost of running a high-volume imaging suite [68]; or improve the existing standard of care sufficiently to justify an increased level of specialist knowledge required.

Translational Characteristic 4. Image Reader Expertise. Converting the raw image data into a relevant OIB is a crucial step, whereby image interpretation criteria must be established. Such criteria need to deliver high sensitivity, specificity, inter-observer agreement and short learning curves. Criteria can include a binary decision, a library-based classification, the presence of specific image patterns $[69,70]$, or a change in signal intensity relative to a defined threshold. Establishing OIBs is a time-consuming task, often requiring international consensus across multiple centres.

For more complex biomarkers, expert image readers need to be trained, which further adds to the cost and time for translation, as well as making the biomarker difficult to standardise across centres. Providing simple feedback of the biomarker, or familiar images that avoid the need for retraining, can enable non-experts to make diagnoses and smooth the translational pathway.

Partially or fully automated analysis provides an attractive means of reducing or removing the burden on expert image readers. It has the potential to be objective, standardised and low-cost, but has yet to mature to stage where it is fully capable of operating in real time with sufficient performance to replace the human image reader [71, 72]. For many optical imaging techniques, data reduction is essential because the dimensionality of the data is beyond interpretation by the human image reader.

Translational Characteristic 5. Repeatability and Reproducibility. Both repeatability (test-retest) and reproducibility across devices, operators and interpreters must be assessed to evaluate the achievable precision for measurement of the OIB. Though inter-observer agreement (encompassing operators and interpreters) is often assessed, the intra-observer, intradevice and inter-device variability are often overlooked, which makes comparative evaluation of novel OIBs challenging. To maximise the opportunity for translation to the intended setting, studies should be designed to enable comparison of results obtained across multiple centres. 
Translational Characteristic 6. Co-registration. A key facet of imaging is the ability to provide spatially resolved information which can be co-registered with the anatomy of the patient to allow guidance for intervention. There are several methods of achieving this with increasing levels of complexity that could hinder translation: co-registration with an existing technique that is compatible with surgical treatment [73]; application of fiducial markers, such as laser cautery marks, to highlight target areas [74]; or projection of the image data onto the patient or into the surgeon's field of view using augmented reality technology $[75,76]$.

\subsection{Outlook}

Imaging is the only medical tool currently capable of non-invasively capturing detailed, immediate and spatially resolved biochemical information in vivo and thus delineating disease such that non-invasive curative resection or treatment can take place. Despite great promise, few optical imaging techniques have been translated to routine clinical use. The translational characteristics we identified complement the OIB Roadmap, which we hope will improve the chances of novel optical imaging techniques being translated to widespread clinical implementation. With such a vast array of complex tissue-light interactions, and an equally diverse arsenal of developing optical devices, some are sure to change the current standard of care for cancer patients, leading to improved outcomes in the future.

This thesis describes recent efforts to advance three optical endoscopic imaging techniques towards clinical translation. It is split into two parts: Chapters 2-5 describe work on two novel flexible endoscopic imaging techniques for early detection of cancer in the oesophagus; Chapter 6 describes work on a novel rigid endoscopic imaging technique for delineation of cancer and healthy tissue in surgery to remove pituitary tumours. For each of these techniques, the following aims were established.

- Guided by the translational characteristics, to develop a novel optical imaging technique capable of swift clinical translation, including development of the device, contrast mechanism and image analysis methods.

- To translate the technique through the beginning of Domain 2 of the OIB Roadmap, including technical characterisation, to ensure the device meets design specifications, and technical validation, to ensure precision, and biological validation, to ensure the device is able to distinguish between the tissues of interest in a range of representative ex vivo samples. 
- To ultimately conduct the first-in-human trials of the technique, including design and approval of a clinical pilot study and local safety approval of the device and contrast mechanism for use in humans.

This thesis concludes with an outlook for the future, outlining the steps required to improve each technique and evaluating their potential to advance further towards clinical translation and to ultimately improve the standard of care in their respective indications.

In the following chapters, reference to the translational characteristics and translational barriers will frequently be made to highlight where their consideration has influenced the successes and failures of optical imaging techniques, as well as to underline where they have influenced the decisions in designing the techniques and experimental methodologies described in this thesis. Reference to the OIB Roadmap shall also be made to emphasise where each stage of the work sits on the path to translation and highlight the experiments required to address the next steps in the roadmap. 



\section{FLEXIBLE ENDOSCOPY: EARLY DETECTION OF DYSPLASIA IN BARRETT'S OESOPHAGUS}

This chapter uses material from a review paper by the author entitled: "Emerging Optical Methods for Endoscopic Barrett's Surveillance" (see publications list).

Early detection of cancer confers more than a three-fold increase in ten-year-survival rate [77], but screening is challenging: it must achieve low false negative rates to avoid missing potentially deadly disease, whilst retaining low false positive rates to prevent over-treatment; it must be low-cost such that it can be widely implemented and crucially, if localised treatment is to be possible, for example by ablation or resection, the screening technique must provide spatially resolved information. Optical imaging has the potential to achieve these goals by capturing spatially resolved biochemical information based on the contrast mechanisms discussed in Chapter 1 and summarised in Figure 1.1.

Though optical imaging is relatively low cost in comparison to radiological imaging, widespread screening regimes can be prohibitively expensive. Fortunately, some cancers are associated with a known precursor disease, enabling us to more affordably perform surveillance on a smaller enriched population, detect the early stages of cancer, non-invasively resect or treat this, and thus improve survival rates. One such disease is Barrett's oesophagus. This chapter describes Barrett's oesophagus, the current standard of care surveillance, and the current state of the art in advanced endoscopic surveillance techniques. 


\subsection{Barrett's Oesophagus}

Barrett's oesophagus is an acquired condition in which columnar epithelium replaces the stratified squamous epithelium of the lining of the distal oesophagus, the pipe which connects the throat to the stomach. Crucially for early detection attempts, Barrett's oesophagus predisposes patients to the development of oesophageal adenocarcinoma (OAC) [78]. The progression to cancer occurs through an intermediate stage known as dysplasia, which can be of low-grade (LGD) or high-grade (HGD). The elevated cancer risk in Barrett's oesophagus is variably reported (Figure 2.1) but is somewhere around $0.1-0.3 \% /$ year in non-dysplastic Barrett's oesophagus [79-81], 9\%/year in the presence of LGD [82] and around 4 times higher in patients harbouring HGD, compared to patients with LGD [83]. As the 5-year survival rate for oesophageal cancer is just $15 \%$, but improves to $80 \%$ when the cancer is identified at an early-stage [84, 85], major advisory bodies recommend that patients with Barrett's oesophagus undergo routine endoscopic surveillance for signs of dysplasia or early carcinoma [86-90] such that they can be treated non-invasively with curative intent.

Indeed, data from some retrospective studies indicate that surveillance correlates with improved survival [91-93], although evidence from a case-controlled study [94] did not confirm this and data from randomised controlled trials is lacking. Still, patients currently undergo surveillance endoscopy every $3-5$ years.

The current standard of care (SOC) for endoscopic surveillance uses high-definition white light endoscopy (HD-WLE) to identify suspicious lesions based on their appearance. Lesions are often difficult to spot, as they are heterogeneous in their shape and size, patchy in their distribution and, especially when flat, show subtle contrast on HD-WLE. Suspicious lesions are biopsied, stained and sectioned to reveal the cell morphology, which is analysed by a pathologist to reach a diagnosis (Figure 2.2).

To mitigate the risk of missing subtle, flat lesions, the SOC protocol also includes taking random biopsies. These are taken at four-quadrant positions circumferentially every $2 \mathrm{~cm}$ along the region of Barrett's oesophagus, a process known as the Seattle protocol, in the hope that this frequent sampling will identify any lesions missed by HD-WLE [86, 95]. The resulting sensitivity is $40 \%-64 \%$ with specificity of $98-100 \%$ [20], but the procedure is costly, timeconsuming and prone to sampling error [90].

The potential to improve clinical outcomes by increasing contrast for dysplasia with advanced optical techniques has driven a great deal of research in this area. With demand for endoscopy predicted to rise substantially over the next decade [96], the unmet clinical need for optical 

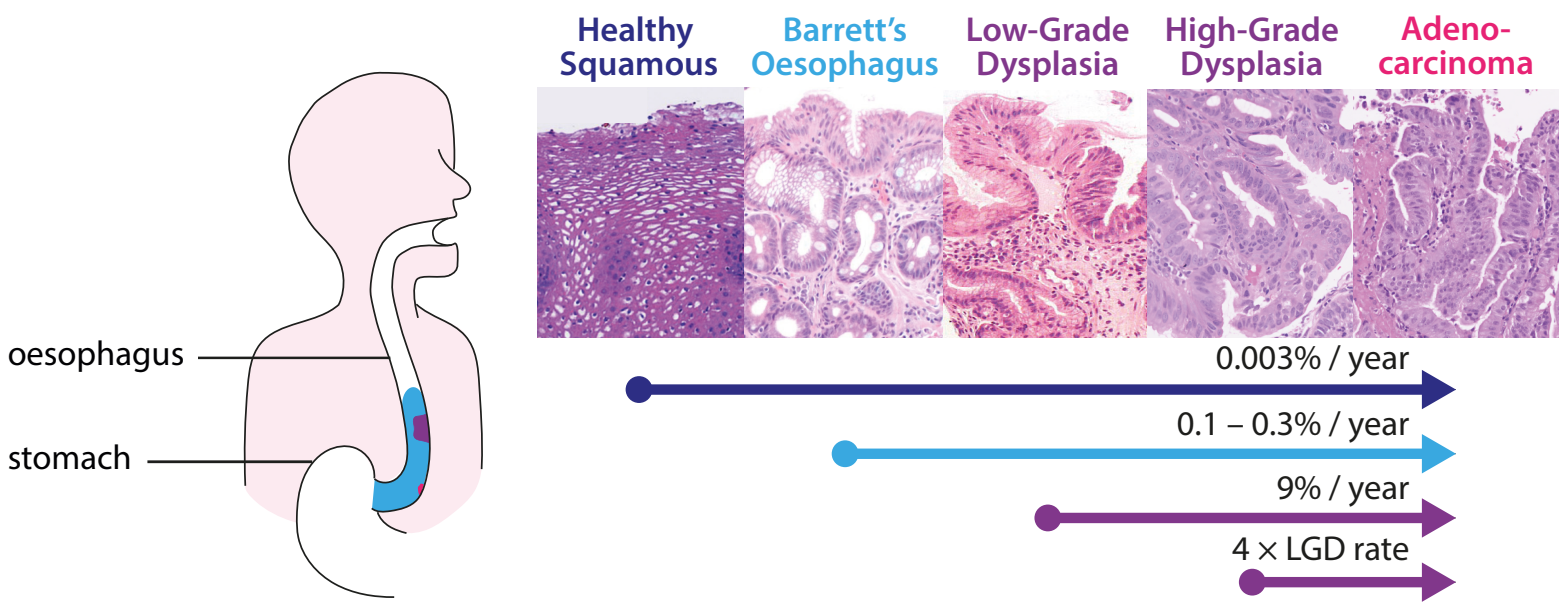

Figure 2.1 Progression of Barrett's oesophagus.

H\&E stained histopathology images illustrate the histopathological cell features that define Barrett's oesophagus, dysplasia and cancer in the distal oesophagus. In Barrett's oesophagus, healthy squamous epithelium is replaced by columnar epithelium. Low-grade dysplasia shows architectural and cytological abnormalities associated with neoplasia, such as crowded crypts and enlarged nuclei. In h dysplasia these are more prominent. In adenocarcinoma, the neoplastic cells penetrate through the basement membrane into the lamina propria. Reproduced from [97]. Progression figures for Barrett's oesophagus are difficult to accurately determine, but estimates are shown [79-83].

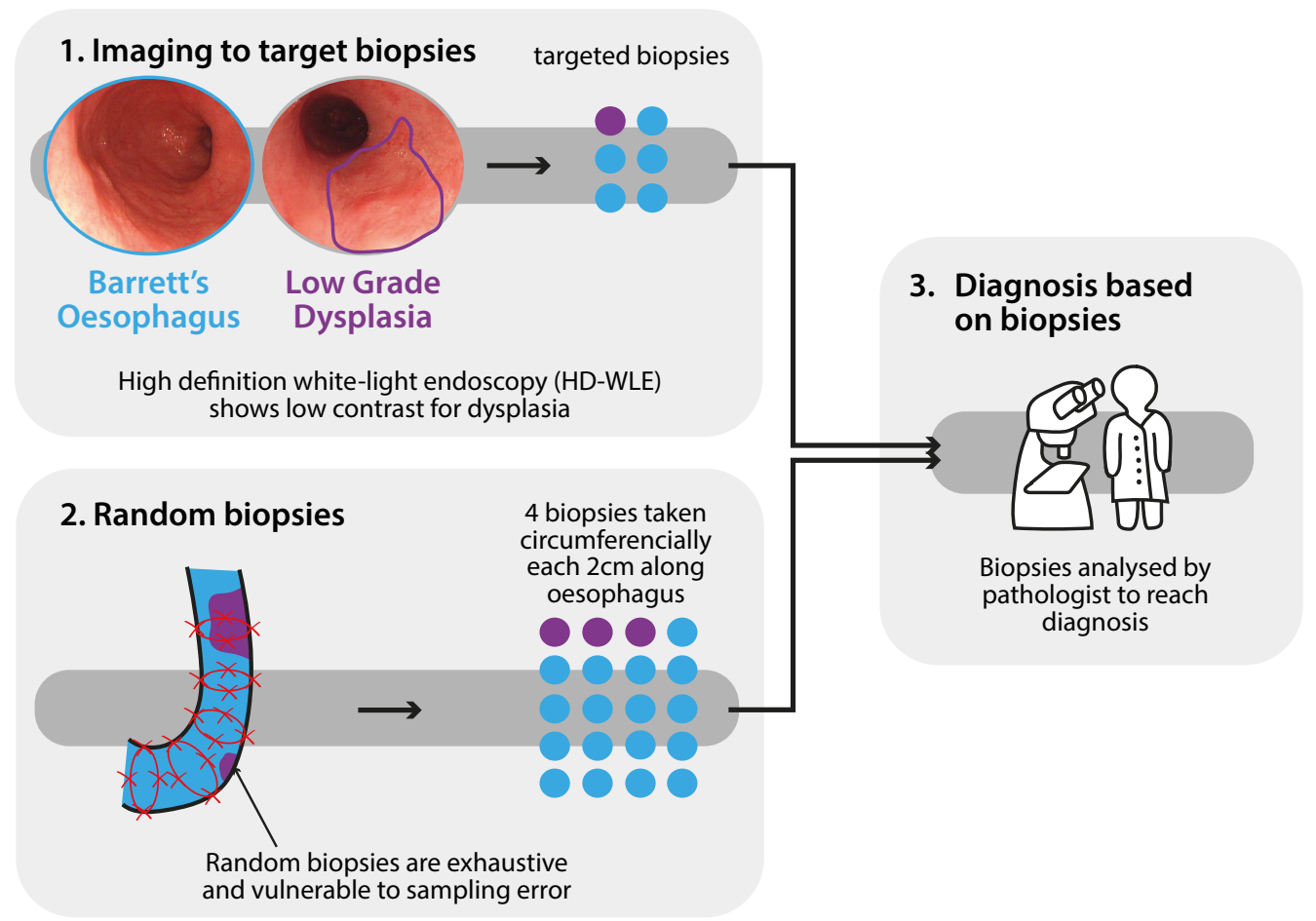

\section{Figure 2.2 Current standard of care for endoscopic surveillance of patients with Barrett's oesophagus.}

Biopsies are targeted by inspection of the oesophagus using high definition white-light endoscopy, but this is limited by poor contrast for dysplasia. Random biopsies are taken at four-quadrant positions circumferentially every $2 \mathrm{~cm}$ along the oesophagus, a process known as the Seattle protocol, in the hope that this frequent sampling will identify any lesions missed by HD-WLE, but these are time consuming, costly and prone to sampling error. Final diagnosis is made from histopathology of the biopsies. These limitations have motivated research into advanced optical techniques for endoscopic surveillance. 
methods with improved diagnostic yield and/or lower cost per procedure is particularly acute. As discussed in Chapter 1.1, harnessing the complex interactions between light and tissue in a clinically translatable optical imaging technique has tremendous potential for non-invasive detection and characterisation of disease tissue, in this case, detection of dysplasia.

\subsection{Improving the Standard of Care}

\subsubsection{Categories of Advanced Optical Endoscopic Techniques}

Advanced optical endoscopic techniques are often categorised as 'red-flag', 'optical biopsy' or 'hybrid' (Figure 2.3). Red-flag techniques provide wide-field images and, if they provide sufficient contrast for dysplasia, can replace both HD-WLE and random four-quadrant biopsies through improved targeting of biopsies (Figure 2.3 (b)). A recent study estimated that using a targeted biopsy protocol without random biopsies could reduce per-patient biopsy costs from $\sim £ 1000$ to $\sim £ 30$ [98]. Conversely, optical biopsy techniques measure a small area of tissue with the goal of providing in vivo, real-time diagnosis (Figure 2.3 (c)). In the short-term, this could reduce the number of biopsies by pre-screening prospective biopsy locations. In the long-term, optical biopsy could replace physical biopsy, enabling immediate diagnosis during surveillance, allowing intervention to occur directly within the same procedure. Hybrid techniques, as the name suggests, combine red flag and optical biopsy capabilities to identify and diagnose disease in vivo.

\subsubsection{Advanced Optical Endoscopic Techniques in Clinical Practice for Barrett's Surveillance}

Several advanced optical techniques are already in clinical use for endoscopic Barrett's surveillance in some centres. Still, endoscopic practice varies significantly across countries and within the same country, and use of these advanced optical techniques is often restricted to tertiary referral centres delivering endoscopic treatment to a high volume of dysplastic patients. A recent meta-analysis by the American Society for Gastrointestinal Endoscopy (ASGE) suggest that to be recommended for targeting biopsy, a new technique should achieve at least $90 \%$ sensitivity, $80 \%$ specificity and $98 \%$ negative predictive value [9].

Table 2.1 shows the recommendation status of techniques currently used in clinic, as well as their key advantages and disadvantages. Figure 2.4 shows example images from each of these techniques. As they have been extensively reviewed elsewhere [99, 100], only a brief summary of each technique is presented here. 


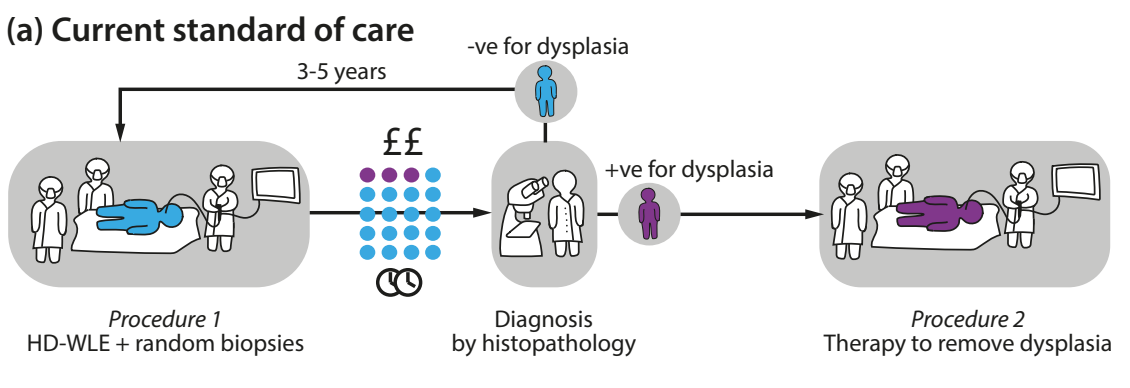

(b) Improved red flag imaging. More accurate targeting so no need for random biopsies.

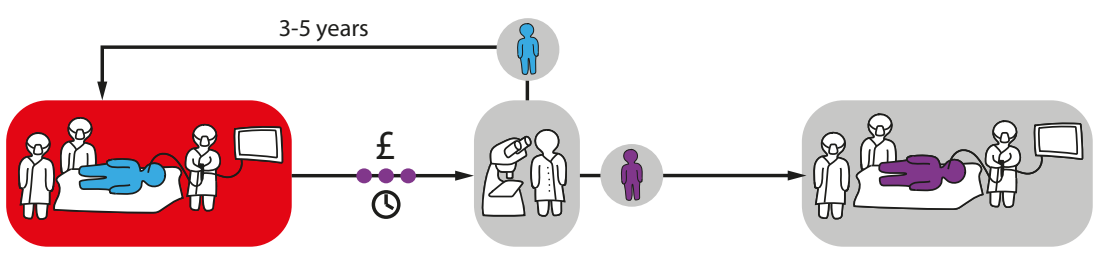

(c) Targeted imaging + optical biopsy or hybrid imaging. Surveillence and treatment occur in a single procedure.

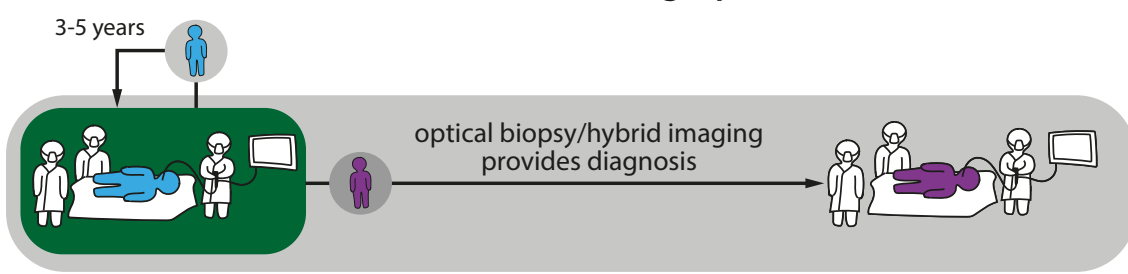

\section{Figure 2.3 'Red flag', 'optical biopsy' and 'hybrid' techniques in relation to the standard of care.}

(a) The current standard of care results in a delay between surveillance endoscopy, diagnosis and treatment, and is costly due to the exhaustive biopsies taken in an attempt to overcome the limitations of red flag imaging with HD-WLE. (b) Improved 'red flag' imaging would survey the oesophagus, identifying suspicious regions with greater sensitivity and specificity than HD-WLE and random biopsies, thus reducing the number of biopsies. (c) 'Optical biopsy' might one day replace physical biopsy providing real time diagnosis in vivo and thus removing the need for physical biopsies and allowing surveillance and treatment to occur in a single procedure. Optical biopsy techniques must be paired with a red flag technique to allow wide field surveillance. Alternatively, 'hybrid' techniques perform the role of both red flag and optical biopsy by scanning the entire oesophagus and providing diagnostic information in vivo. 


\section{Table 2.1 Advanced optical techniques in clinical use for endoscopic surveillance of Barrett's oesophagus.}

Example images for each technique are shown in Figure 2.4. BSG, British Society of Gastroenterology; ASGE, American Society for Gastrointestinal Endoscopy; ACG, American College of Gastroenterology; AGA, American Gastroenterology Association; ESGE, European Society of Gastrointestinal Endoscopy. . Cannot be advocated or discouraged at this time. *The ASGE systematic review and meta-analysis indicates that these modalities meet the preservation and incorporation of valuable endoscopic innovations criteria, but they are not necessarily recommended in routine clinical practice[9]. HD-WLE, high definition white light endoscopy; 4QB, 4 quadrant biopsies according to the Seattle protocol; NBI, narrow band imaging; BLI, blue laser imaging; FICE, Fuji intelligent chromoendoscopy; iSCAN is a brand name used by Pentax to refer to their image enhancement technology.

\begin{tabular}{|c|c|c|c|c|c|c|c|c|c|}
\hline \multirow{2}{*}{ Technique } & \multirow{2}{*}{ Advantages } & \multirow{2}{*}{ Disadvantages } & \multirow{2}{*}{ Sensitivity } & \multirow{2}{*}{ Specificity } & \multicolumn{5}{|c|}{ Recommendation } \\
\hline & & & & & BSG & ASGE $^{*}$ & ACG & AGA & ESGE \\
\hline $\begin{array}{l}\text { HD-WLE + 4QB } \\
\text { and histopathology } \\
\text { of biopsies }\end{array}$ & $\begin{array}{l}\text { Widely available; well } \\
\text { established. }\end{array}$ & $\begin{array}{l}\text { Prone to sampling error } \\
\text { [90]; exhaustive biopsies } \\
\text { are expensive. }\end{array}$ & $\begin{array}{l}0.40-0.68 \\
{[20]}\end{array}$ & $\begin{array}{l}0.98-1.00 \\
{[20]}\end{array}$ & $\checkmark$ & $\checkmark$ & $\checkmark$ & $\checkmark$ & $\checkmark$ \\
\hline Chromoendoscopy & $\begin{array}{l}\text { Inexpensive; widely } \\
\text { available; acetic acid has } \\
\text { shown high sensitivity and } \\
\text { specificity for detecting } \\
\text { dysplasia [9]. }\end{array}$ & $\begin{array}{l}\text { Potential toxicology issues } \\
\text { [101] (methylene blue); } \\
\text { increase in procedure time } \\
\text { [9]; low inter-observer } \\
\text { agreement; no current } \\
\text { procedural terminology } \\
\text { for billing and } \\
\text { reimbursement [9]; } \\
\text { difficulty in achieving } \\
\text { uniform application of dye } \\
\text { [90]. }\end{array}$ & $\begin{array}{l}0.92-0.97 \\
\text { (acetic } \\
\text { acid) } \\
0.642 \\
\text { (methylene } \\
\text { blue) } \\
{[9,102]}\end{array}$ & $\begin{array}{l}0.85-0.96 \\
\text { (acetic } \\
\text { acid) } \\
0.96 \\
\text { (methylene } \\
\text { blue) } \\
{[9,102]}\end{array}$ & $x$ & $\begin{array}{c}\boldsymbol{V} \\
\text { (acetic } \\
\text { acid) }\end{array}$ & $x$ & $x$ & $x$ \\
\hline $\begin{array}{l}\text { Hardware-based } \\
\text { Virtual } \\
\text { Chromoendoscopy } \\
\text { e.g. NBI, BLI }\end{array}$ & $\begin{array}{l}\text { Ability to visualise } \\
\text { mucosal and vascular } \\
\text { patterns; widely available; } \\
\text { ease of use; high } \\
\text { sensitivity and specificity } \\
\text { for detecting dysplasia [9]; } \\
\text { reduced number of } \\
\text { biopsies [103]. }\end{array}$ & $\begin{array}{l}\text { No universal classification } \\
\text { criteria until recent BING } \\
\text { criteria [69]; low inter- } \\
\text { observer agreement; low } \\
\text { sensitivity for low-grade } \\
\text { dysplasia [104]. }\end{array}$ & 0.94 [9] & 0.98 [9] & $x$ & $\checkmark$ & $x$ & $\cdot \cdot$ & $x$ \\
\hline $\begin{array}{l}\text { Software-based } \\
\text { Virtual } \\
\text { Chromoendoscopy } \\
\text { e.g. FICE, iSCAN }\end{array}$ & $\begin{array}{l}\text { No additional hardware } \\
\text { costs. }\end{array}$ & Lack of data. & $\begin{array}{l}0.83 \text { [105] } \\
\text { (high-grade } \\
\text { dysplasia } \\
\text { with FICE) }\end{array}$ & Unavailable & $x$ & $x$ & $x$ & .. & $x$ \\
\hline $\begin{array}{l}\text { Autofluorescence } \\
\text { Imaging } \\
\text { (AFI) }\end{array}$ & $\begin{array}{l}\text { Easy to combine with NBI } \\
\text { and WLE. }\end{array}$ & $\begin{array}{l}\text { Many studies biased by } \\
\text { comparison with } \\
\text { substandard WLE [106]. } \\
\text { Limited value in routine } \\
\text { surveillance [106]. }\end{array}$ & $\begin{array}{l}0.50[107] \\
\text { (high-grade } \\
\text { dysplasia) }\end{array}$ & $\begin{array}{l}0.61[107] \\
\text { (high-grade } \\
\text { dysplasia) }\end{array}$ & $x$ & $x$ & $x$ & $x$ & $x$ \\
\hline $\begin{array}{l}\text { Endoscopic } \\
\text { Trimodal Imaging } \\
\text { (ETMI) }\end{array}$ & $\begin{array}{l}\text { Reduced false positive } \\
\text { rate relative to AFI alone. }\end{array}$ & $\begin{array}{l}\text { Useful in tertiary referral } \\
\text { centres [62] but not in } \\
\text { community practice [63]. }\end{array}$ & $0.81[9]$ & $0.46[9]$ & $x$ & $x$ & $x$ & $x$ & $x$ \\
\hline $\begin{array}{l}\text { Probe-based } \\
\text { Confocal Laser } \\
\text { Endomicroscopy } \\
\text { (pCLE) }\end{array}$ & $\begin{array}{l}\text { Probe can be inserted } \\
\text { through working channel } \\
\text { of standard endoscope; } \\
\text { close to in-vivo histology. }\end{array}$ & $\begin{array}{l}\text { Often uses exogenous } \\
\text { contrast (fluorescein). }\end{array}$ & $0.90[9]$ & 0.77 [9] & $x$ & $x$ & $x$ & $x$ & $x$ \\
\hline $\begin{array}{l}\text { Endoscope-based } \\
\text { Confocal Laser } \\
\text { Endomicroscopy } \\
\text { (eCLE) }\end{array}$ & $\begin{array}{l}\text { Close to in-vivo histology; } \\
\text { high sensitivity and } \\
\text { specificity for detecting } \\
\text { dysplasia [9]. }\end{array}$ & $\begin{array}{l}\text { Requires dedicated } \\
\text { endoscope (in contrast to } \\
\text { pCLE); often uses } \\
\text { exogenous contrast } \\
\text { (fluorescein). }\end{array}$ & $0.90[9]$ & 0.93 [9] & $x$ & $\checkmark$ & $x$ & $x$ & $x$ \\
\hline
\end{tabular}


(a)

(b)

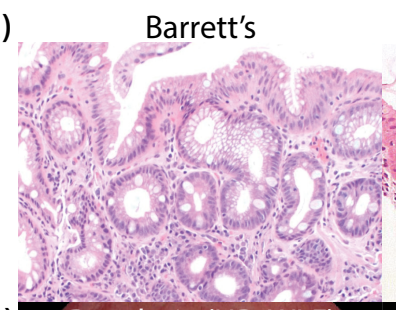

(c)

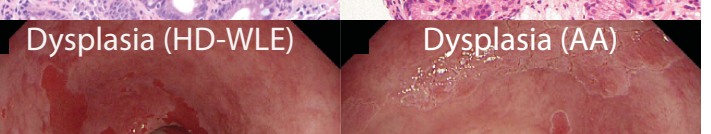

(d)

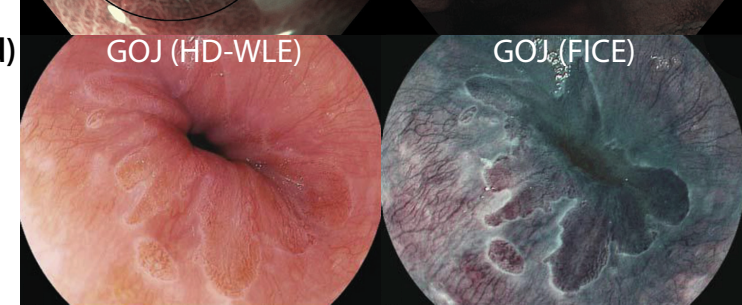

(e)

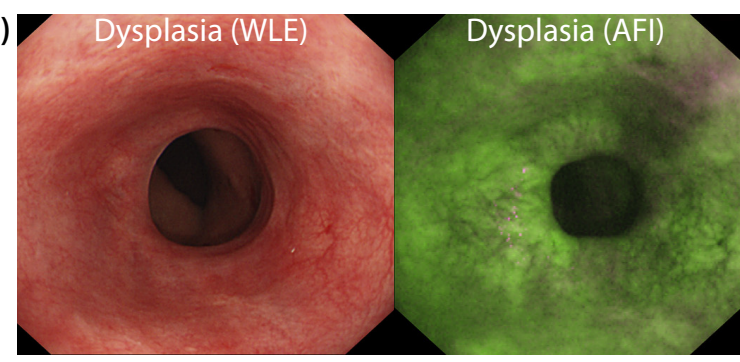

(f)

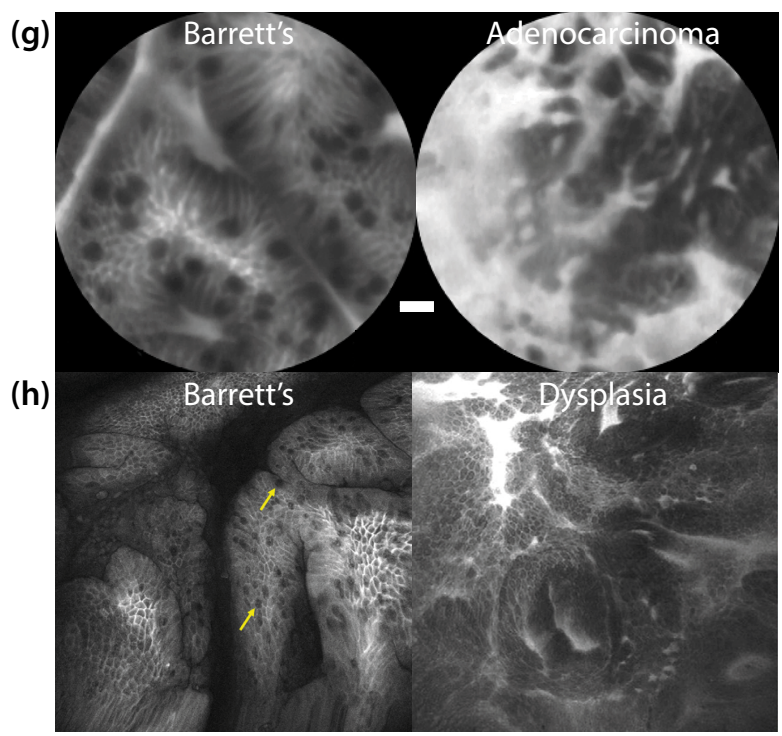

Figure 2.4 Advanced optical techniques in clinical use for endoscopic surveillance of Barrett's oesophagus - example images.

(a) Histopathology: Left: Non-dysplastic Barrett's oesophagus. Right: Low-grade dysplasia. (b) Chromoendoscopy, Acetic Acid: Left: Barrett's with HD-WLE. Right: Same patient. Note dysplasia only visible post acetic acid (AA) with early loss of acetowhitening (red patch on the lumen in the lower side of the image) (Olympus Lucera ELITE processor, GIFHQ290 gastroscope). Reproduced from [98]. (c) Hardware-based Virtual Chromoendoscopy, NBI: Left: Barrett's oesophagus. Note the presence of circular (solid black arrow) and ridge/villous (red arrow) mucosal patterns arranged in an orderly fashion and blood vessels that follow the mucosal ridge architecture (dashed arrows). Reproduced from [69]. Right: Dysplasia. Note the irregular mucosal (black arrow) and vascular patterns (red arrow). Reproduced from [69]. (d) Software-based Virtual Chromoendoscopy, FICE: Left: Conventional white-light image of the gastroesophageal junction (GOJ) (from FICE ATLAS, Fujicon). Right: Fujicon intelligent chromoendoscopy (FICE) image of the GOJ (From FICE ATLAS Fujicon). Reproduced from [8]. (e) Autofluorescence Imaging: Left: High-grade dysplasia not visible on WLE. Reproduced from [106]. Right: AFI reveals high-grade dysplasia as a purple patch. Reproduced from [106]. (f) Endoscopic Trimodal Imaging: See HD-WLE (b), AFI (e), NBI (c). (g) pCLE: Left: Barrett's oesophagus. Right: Early oesophageal adenocarcinoma. Images captured in vivo using GastroFlex UHD (Cellvizio; Mauna Kea Technologies, Paris, France) after injection of sodium fluorescein $(2.5 \mathrm{~mL}, 10 \%)$. Scale bar $=20 \mu \mathrm{m}$. Reproduced from [108]. (h) eCLE: Confocal images of the upper part of the mucosa layer (about 30$50 \mu \mathrm{m}$ vertical depth). Left: Barrett's epithelium. Typical villiform shape and presence of goblet cells (yellow arrows) can be identified. Right: High-grade dysplasia. At confocal images black cells with irregular borders and shapes with high dark contrast to surrounding tissue were present. Images captured in vivo with confocal laser endoscope (EC-3870CIFK; Pentax, Tokyo, Japan) after injection of 10\% fluorescein sodium (5-10 ml IV). Reproduced from [109]. 


\section{A. Chromoendoscopy (red flag)}

Chromoendoscopy enhances contrast through the use of topically applied dyes. Acetic acid eliminates the superficial mucosal layer and then causes changes to (acetylation of) cellular proteins, resulting in whitening that highlights surface patterns. In the case of neoplastic Barrett's, this is rapidly followed by focal erythema (redness) caused by vascular congestion in capillaries, which is revealed as focal redness as loss of acetowhitening occurs [98]. These reactions are used to guide targeted biopsies and increase the yield of dysplasia, meeting the ASGE performance thresholds [9, 110]. Methylene blue chromoendoscopy has also been extensively investigated, but there are concerns regarding possible carcinogenic effects of the dye [101]. Meta-analyses have found it to be inferior to WLE [111] and acetic acid chromoendoscopy [9]. It is therefore likely that acetic acid will become the standard conventional chromoendoscopic method for Barrett's surveillance.

\section{B. Virtual chromoendoscopy (red flag)}

Virtual (also known as electronic or optical) chromoendoscopy improves contrast by modifying the endoscope hardware or software. This avoids the challenges of working with dyes, such as increased procedure time for dye administration and potential for adverse effects caused by the dye (Translational Characteristic 5). Hardware modifications reported to date usually involve adapting the light source to focus on blue and green wavelength bands, where haemoglobin is strongly absorbing, hence providing contrast based on changes in the tissue vasculature [99]. Narrow band imaging (NBI) is the most widely established of these methods and also meets the ASGE thresholds [9]. NBI highlights the mucosal and vascular pattern of the superficial mucosa, enabling the operator to classify the disease state of the tissue based on recognition of altered vascular and mucosal patterns associated with dysplasia [69]. Blue laser imaging (BLI) is a similar technology that has also been tested in patients (in vivo, comparative study, $\mathrm{n}=39$ patients) [112] and is under evaluation in Barrett's oesophagus [113]. Software-based virtual chromoendoscopy methods $[114,115]$ use proprietary image processing algorithms to improve the contrast of mucosal and surface vessel patterns in the GI tract [8]. While there is currently insufficient data for advisory bodies to make recommendations [9], clinical studies have shown that software-based approaches perform similarly to acetic acid chromoendoscopy (in vivo, prospective randomized pilot study, $n=57$ ) [105]. These early findings need to be confirmed with large randomised controlled trials. Virtual chromoendoscopy has significant advantages in being label-free and easily implemented using SOC devices (Translational Characteristic 6) [116-118], so now has widespread availability. 


\section{Autofluorescence endoscopy (red flag)}

Several endogenous structural and metabolic molecules, such as collagen and NADH, are fluorescent. This 'autofluorescence' is reduced in dysplastic tissue compared to the surrounding healthy tissue, providing contrast for autofluorescence imaging (AFI). In commercial endoscopes with AFI, dysplastic tissue is displayed as dark purple patches among a background of green Barrett's fluorescence (Figure 2.4) [119]. This has high sensitivity for dysplasia, but low specificity since inflammation also reduces tissue autofluorescence [120]. AFI is implemented by adding filters to the light source and detector on a standard endoscope, so it has been combined with HD-WLE as well as virtual chromoendoscopy for endoscopic trimodal imaging (ETMI) in an effort to increase specificity. Trials to date have yielded mixed results $[62,63]$; it remains unclear whether AFI truly adds to the already improved performance of NBI. Like NBI, AFI is widely available, potentially due to its incorporation into standard HDWLE devices (Translational Characteristic 6).

\section{Endomicroscopy (optical biopsy)}

Intravenous fluorescein (a fluorescent dye) can be used to highlight microvasculature and tissue structures. This is commonly examined using confocal laser endomicroscopy (CLE), which produces depth-sectioned, high magnification and high resolution images. These can be used to spot dysplastic changes in cell morphology, for example, irregular vessels or epithelial borders, yielding high sensitivity and specificity for dysplasia $[9,121]$. An endoscope-based CLE system (eCLE) was recommended by the ASGE [9], but requires a dedicated endoscope that is no longer on the market. Probe-based CLE (pCLE) uses a thin fibre bundle allowing the probe to be inserted through the working channel of a standard forward facing endoscope. This allows pCLE to occur alongside standard HD-WLE (Translational Characteristic 2) but with lower resolution and limited depth sectioning compared to eCLE. Clinical trial results to date indicate that pCLE can be used to identify neoplasia but is not yet sufficient to replace random biopsies [122].

\subsubsection{Emerging Optical Endoscopic Techniques for Barrett's Surveillance}

The advanced imaging techniques described above are available in clinically approved devices now used in some centres, and three of these techniques, acetic acid chromoendoscopy, NBI and eCLE were recently reported to meet the ASGE performance requirements for targeting biopsy [9]. The eCLE device has since been removed from the market but for NBI and acetic acid chromoendoscopy, this milestone was achieved in part thanks to their having many of the translational characteristics we suggested in Chapter 1. Their deployment at many sites 
internationally enabled the extensive development of image classification criteria, the publication of consensus statements and the assessment of repeatability and reproducibility (Translational Characteristic 5, Translational Characteristic 4) [59-61, 69, 110, 123]. Both techniques are also compatible with standard forward facing endoscopes, producing familiar 2D images which can be co-registered with HD-WLE (Translational Characteristic 2, Translational Characteristic 6).

These techniques, then, represent the potential to reach widespread implementation by ensuring favourable translational characteristics, as well as performance. Nonetheless, though they are widely used as adjuncts to HD-WLE, neither has yet replaced the standard of care and their recommendation status still hinges on their deployment by expert endoscopists (Translational Characteristic 3, Translational Characteristic 4). Thus, development of novel optical imaging techniques continues, with advances being made that could address outstanding limitations in performance and translatability. These emerging optical techniques are summarised in Table 2.2, with example data from their application in oesophageal tissue shown in Figure 2.5 [20, 124].

\section{A. Interrogating disordered tissue structure}

HD-WLE interrogates disordered tissue structure by presenting images of macroscopic abnormalities on the epithelial surface. Recent advances allow endoscopists to probe crosssectional tissue microstructure, up to several millimetres deep. Optical coherence tomography (OCT) uses scanning low-coherence interferometry to construct cross sectional reflectance images that reveal changes in tissue microstructure arising due to variations in light scattering [125]. Using a set of manual interpretation criteria, such as presence of glands in the images, gives excellent contrast for dysplasia $[126,127]$. Wide-field endoscopic applications of OCT were made feasible by the shift from time-domain OCT to optical frequency domain imaging (OFDI), which significantly increased data acquisition rates and allowed 3D images of the entire oesophagus to be acquired using helical scanning inside an inflated balloon to centre the device, a technique often referred to as volumetric laser endomicroscopy (VLE), which is now commercially available [128]. OCT probes may also be housed in tethered capsule endoscopes (Chapter 3), which can be swallowed un-sedated under the supervision of a nurse in primary care, reducing the required expertise (Translational Characteristic 3) [68, 129, 130].

VLE has been successfully correlated with histology in Barrett's patients (ex vivo, feasibility study, $\mathrm{n}=14$ matched resection specimens) [131] and has been shown to detect oesophageal neoplasia in vivo (in vivo, patient series, $\mathrm{n}=6$ patients) [127]. Recently, endoscopic OCT has also been used to visualise $3 \mathrm{D}$ subsurface microvasculature in the oesophagus [132]. OCT 
angiography images were interpreted using two microvascular features, abnormal branching and heterogenous vessel size, to allow recognition of dysplasia with $94 \%$ sensitivity and $69 \%$ specificity (in vivo, $\mathrm{n}=52$ patients) [133].

A major challenge of OCT-based techniques is that they cannot be used alongside biopsy tools with the existing device architectures (Translational Characteristic 2), so laser cautery marking is being investigated to safely mark regions of interest for later biopsy under HD-WLE guidance [74] (in vivo, pilot study, $\mathrm{n}=22$ patients). A second challenge remains with image interpretation; an experienced OCT endoscopist is currently needed to interpret images, limiting widespread deployment, but automated image analysis is being investigated to alleviate this burden (Translational Characteristic 4) [134].

In addition to providing contrast for OCT, variation in light absorption and scattering from tissue can be recorded as a function of wavelength or angle. Diffuse reflectance spectroscopy (DRS), also called elastic scattering spectroscopy (ESS), illuminates the tissue with a standard white light source, but instead of collecting an image of the oesophagus using a camera, changes in the spectrum of the light arising from absorption and scattering events in the superficial layers of the tissue are measured with a spectrometer. Contact (ESS) [135] and fixed-distance (DRS) [136] probes can differentiate between healthy and dysplastic tissue in the oesophagus, though are typically restricted to point-based measurements rather than wide-field imaging.

Taking the concept a step further, light scattering spectroscopy (LSS) singles out reflected light that has only scattered once in tissue. The benefit of this approach is that LSS measurements can be directly linked to tissue morphology via physical Mie scattering theory, enabling quantitative measurements of the size and density of cell nuclei, which are typically enlarged and crowded in early cancer [137]. Early LSS studies achieved around 90\% sensitivity and specificity for oesophageal dysplasia (in vivo, single centre pilot study, $\mathrm{n}=13$ patients, $\mathrm{n}=76$ sample positions) [138] with a contact probe, but unwanted variations in probe-tissue separation led to challenges for interpretation. Hardware developments overcame this limitations to enable $8 \mathrm{~cm}$ segments of oesophagus to be mapped with $92 \%$ sensitivity and $96 \%$ specificity (in vivo, single centre pilot study, $n=9$ patients, $n=95$ biopsies) [139], showing potential for this to become a useful red-flag tool for guiding targeted biopsies in Barrett's surveillance. 


\section{Table 2.2 Emerging optical techniques for endoscopic surveillance of Barrett's oesophagus.}

Example images for each technique are shown in Figure 2.5. ESS, elastic scattering spectroscopy; DRS, diffuse reflectance spectroscopy; LSS, light scattering spectroscopy.

\begin{tabular}{|c|c|c|c|}
\hline Technique & Contrast mechanism & Strengths and weaknesses & $\begin{array}{l}\text { Status and prospect in } \\
\text { Barrett's oesophagus }\end{array}$ \\
\hline \multicolumn{4}{|l|}{ Exogenous contrast } \\
\hline $\begin{array}{l}\text { Optical Molecular } \\
\text { Imaging } \\
\text { (OMI) }\end{array}$ & $\begin{array}{l}\text { Exogenous fluorophores conjugated to } \\
\text { targeting moieties (lectins, peptides, } \\
\text { antibodies, affibodies, enzymes) that } \\
\text { target intracellular and extracellular } \\
\text { proteins and enzymes. }\end{array}$ & $\begin{array}{l}\text { + Specificity. } \\
\text { - Exogenous contrast. } \\
\text { - Surface images. } \\
\text { - Cost. }\end{array}$ & $\begin{array}{l}\text { In vivo trials [140]. } \\
\text { Potential to be translated for wide } \\
\text { field surveillance. Awaiting } \\
\text { further in vivo trials. }\end{array}$ \\
\hline \multicolumn{4}{|c|}{ Interrogating disordered tissue microstructure } \\
\hline $\begin{array}{l}\text { Optical Coherence } \\
\text { Tomography } \\
\text { (OCT) }\end{array}$ & $\begin{array}{l}\text { Structural features cause change of } \\
\text { phase which can be used to construct } \\
\text { cross sectional image of tissue structure. }\end{array}$ & $\begin{array}{l}\text { + High resolution. } \\
+ \text { Depth sectioning. } \\
+ \text { Endogenous contrast. } \\
\text { - Large image datasets. }\end{array}$ & $\begin{array}{l}\text { In vivo trials (patient series, } \mathrm{n}=6 \text { ) } \\
{[127] \text {. }}\end{array}$ \\
\hline $\begin{array}{l}\text { Elastic Scattering } \\
\text { Spectroscopy } \\
\text { (ESS/DRS/LSS) }\end{array}$ & $\begin{array}{l}\text { Endogenous scatterers (organelles, cell } \\
\text { nuclei, cell membrane, collagen, } \\
\text { cytoplasm, lipids). }\end{array}$ & $\begin{array}{l}\text { + Depth penetration. } \\
\text { + Endogenous contrast. } \\
\text { - Spectrum rather than image. }\end{array}$ & $\begin{array}{l}\text { In vivo trials (single centre pilot } \\
\text { study, } \mathrm{n}=9 \text { patients, } \mathrm{n}=95 \\
\text { biopsies) [139]. No trials } \\
\text { published in last } 10 \text { years. }\end{array}$ \\
\hline $\begin{array}{l}\text { Angle-resolved } \\
\text { Low Coherence } \\
\text { Interferometry } \\
\text { (a/LCI) }\end{array}$ & $\begin{array}{l}\text { Endogenous scatterers (organelles, cell } \\
\text { nuclei, cell membrane, collagen, } \\
\text { cytoplasm, lipids). Increase in nuclear } \\
\text { size with dysplasia can be inferred. }\end{array}$ & $\begin{array}{l}\text { + High sensitivity and specificity in pilot } \\
\text { study. } \\
+ \text { Endogenous contrast. } \\
- \text { Tissue orientation can affect results. }\end{array}$ & $\begin{array}{l}\text { In vivo pilot study ( } 2 \text { centre pilot } \\
\text { study, } \mathrm{n}=46 \text { patients, } \mathrm{n}=172 \text { sites }) \\
\text { [141]. Combination with OCT. } \\
\text { Clinical trials likely. }\end{array}$ \\
\hline Polarimetry & Microstructural anisotropy. & $\begin{array}{l}\text { + Endogenous contrast. } \\
\text { - Instrumentation challenges. }\end{array}$ & $\begin{array}{l}\text { No trials. Awaiting further device } \\
\text { development. }\end{array}$ \\
\hline \multicolumn{4}{|c|}{ Interrogating abnormal tissue function } \\
\hline $\begin{array}{l}\text { Photoacoustic } \\
\text { Endoscopy } \\
\text { (PAE) }\end{array}$ & $\begin{array}{l}\text { Absorption of endogenous } \\
\text { chromophores (melanin, haemoglobin, } \\
\text { lipids, water). Allows vascular imaging. }\end{array}$ & $\begin{array}{l}\text { + Volumetric images. } \\
+ \text { Endogenous contrast. } \\
\text { - Instrumentation challenges. } \\
\text { - Limited resolution at present. } \\
\text { - Long acquisition times. }\end{array}$ & $\begin{array}{l}\text { In vivo imaging of oesophagus in } \\
\text { animals [142]. No trials. } \\
\text { Awaiting application. }\end{array}$ \\
\hline $\begin{array}{l}\text { Fluorescence } \\
\text { Lifetime Imaging } \\
\text { (FLIM) }\end{array}$ & $\begin{array}{l}\text { Endogenous fluorophores (NAD }(\mathrm{P}) \mathrm{H} \text {, } \\
\text { flavins, collagen, elastin). }\end{array}$ & $\begin{array}{l}\text { + More robust than traditional AFI. } \\
\text { + Endogenous contrast. } \\
\text { - Safety of UV illumination. } \\
\text { - Long acquisition times. }\end{array}$ & $\begin{array}{l}\text { Ex vivo trials (time-resolved } \\
\text { fluorescence, single centre pilot } \\
\text { study, } n=37 \text { patients, } n=108 \\
\text { fluorescence decay profiles) } \\
\text { [143]. Awaiting in vivo trials. }\end{array}$ \\
\hline $\begin{array}{l}\text { Multi-photon } \\
\text { Microscopy } \\
\text { (MPM) }\end{array}$ & $\begin{array}{l}\text { Endogenous fluorophores (NAD }(\mathrm{P}) \mathrm{H} \text {, } \\
\text { flavins, collagen, elastin). }\end{array}$ & $\begin{array}{l}+ \text { Depth sectioning. } \\
+ \text { High resolution. } \\
+ \text { Endogenous contrast. } \\
\text { - Requires high illumination intensity. }\end{array}$ & $\begin{array}{l}\text { Ex vivo trials }(\mathrm{n}=25 \text { patients, } \\
\mathrm{n}=35 \text { biopsies })[144] \\
\text { Awaiting in vivo trials. }\end{array}$ \\
\hline \multicolumn{4}{|c|}{ Interrogating bulk molecular composition } \\
\hline $\begin{array}{l}\text { Endoscopic } \\
\text { Raman } \\
\text { Spectroscopy } \\
\text { (ERS) }\end{array}$ & $\begin{array}{l}\text { Vibrational modes of specific molecules } \\
\text { including proteins (proteins, RNA, } \\
\text { DNA, lipids, water). }\end{array}$ & $\begin{array}{l}\text { + Detailed biochemical information. } \\
+ \text { Algorithms have been developed } \\
+ \text { Multicentre trials underway. } \\
+ \text { Endogenous contrast. } \\
\text { - Spectrum rather than image. } \\
\text { - Repeatability has been questioned. } \\
\text { - Validation using ex vivo tissue is } \\
\text { difficult. }\end{array}$ & $\begin{array}{l}\text { In vivo trials (pilot study, } \mathrm{n}=77 \\
\text { patients) [97]. } \\
\text { Potential to be translated if } \\
\text { repeatability can be confirmed. } \\
\text { Awaiting multicentre trials. }\end{array}$ \\
\hline $\begin{array}{l}\text { Coherent anti- } \\
\text { Stokes Raman } \\
\text { Spectroscopy } \\
\text { (CARS) }\end{array}$ & Specific molecular groups (see ERS). & $\begin{array}{l}\text { + Detailed biochemical information. } \\
\text { + Increased sensitivity compared to ERS. } \\
\text { - Instrumentation challenges. } \\
\text { - Requires high illumination intensity. }\end{array}$ & $\begin{array}{l}\text { No trials. Awaiting further device } \\
\text { development. }\end{array}$ \\
\hline \multicolumn{4}{|l|}{ Multimodal methods } \\
\hline $\begin{array}{l}\text { Multispectral } \\
\text { Imaging } \\
\text { (MSI) }\end{array}$ & $\begin{array}{l}\text { Endogenous chromophores (melanin, } \\
\text { haemoglobin, lipids, water). } \\
\text { Endogenous fluorophores (NAD(P)H, } \\
\text { flavins, collagen, elastin). }\end{array}$ & $\begin{array}{l}\text { + Simple. } \\
+ \text { Compact. } \\
+ \text { Endogenous contrast. } \\
\text { - Surface images. }\end{array}$ & No trials. Awaiting application. \\
\hline
\end{tabular}


(a)
(b)
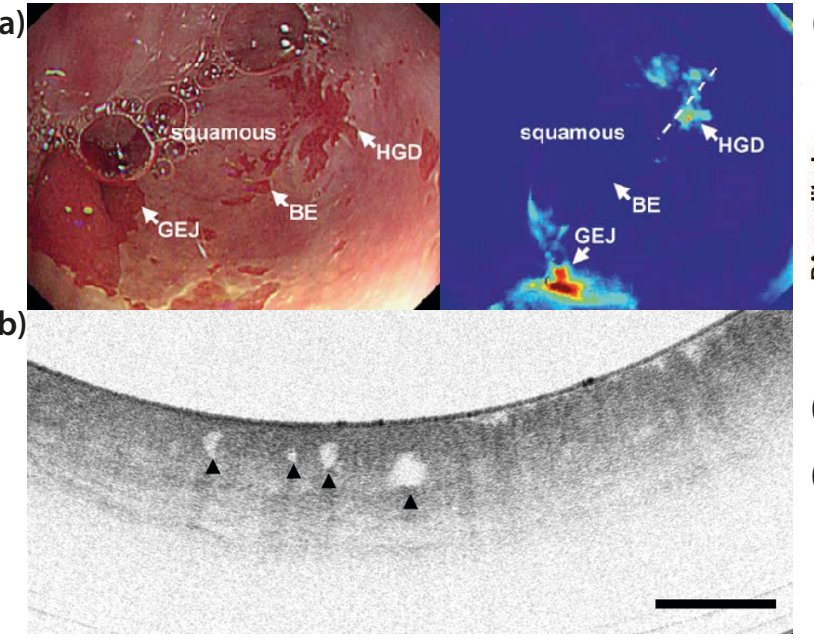

(c)

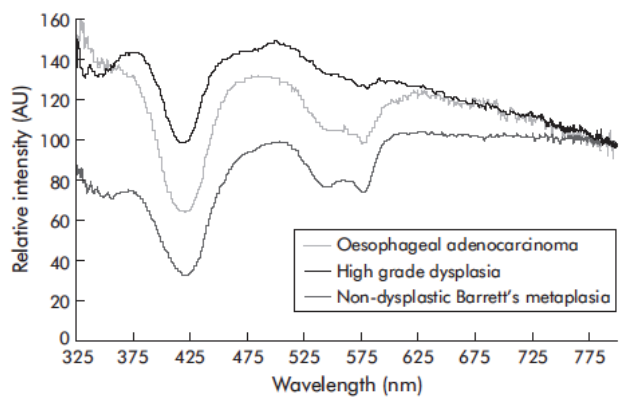

(d)

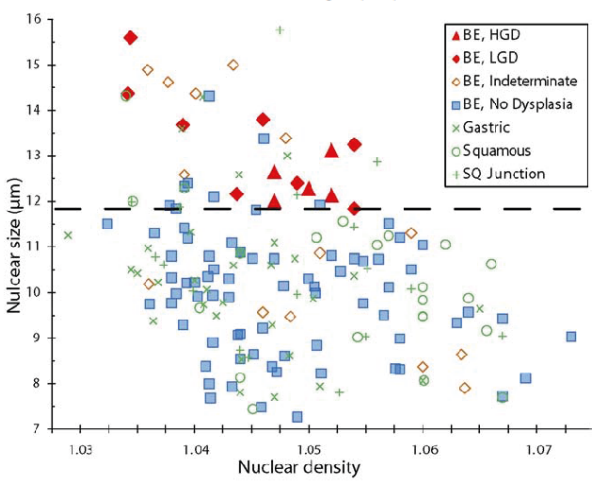

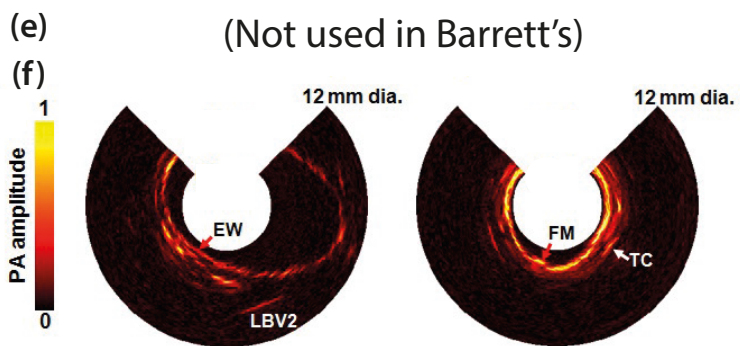

(g)

(h)

(i)

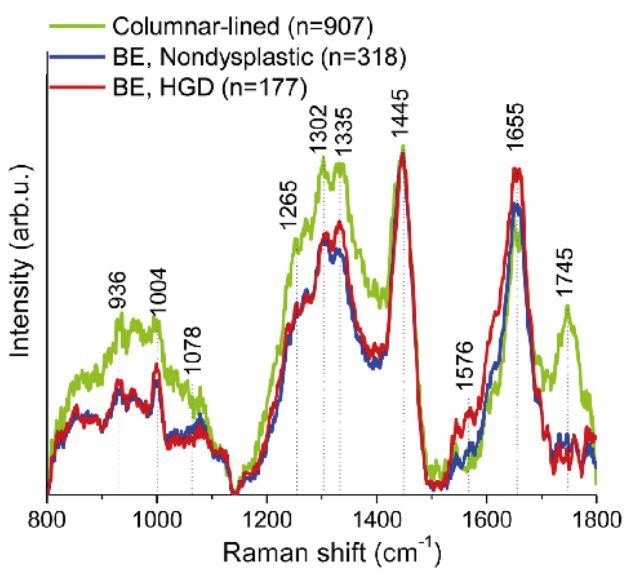

(j)

(k)
(Not used in Barrett's)

(Not used in Barrett's)
(Not used in Barrett's)

(Not used in Barrett's)

Figure 2.5 Emerging optical techniques for endoscopic surveillance of Barrett's oesophagus - example images/spectra.

(a) OMI: Left: White-light image showing several areas of Barrett's oesophagus (labelled BE in the diagram), identified by salmon-red mucosa, surrounded by squamous epithelium but no macroscopically visible structural abnormalities to suggest the presence of neoplasia. Right: Targeted fluorescence image showing enhancement of the signal from HGD. Reproduced from [145]. (b) OCT: Features consistent with dysplasia, including irregular glandular architecture (arrowheads). Captured with NvisionVLE Imaging System. Scale bar=1 mm. Reproduced from [146]. (c) ESS/DRS/LSS: Representative spectra obtained with elastic scattering spectroscopy in vivo. AU: arbitrary units. Reproduced from [135]. (d) a/LCI: Scatter plot with each biopsy plotted as a function of its nuclear size and density, as measured by a/LCI system in vivo, and categorised by its pathological diagnosis. Reproduced from [141]. (e) Polarimetry: Not used in Barrett's. (f) PAE: B-scan images of in vivo rabbit oesophagus. Each image covers a $12 \mathrm{~mm}$ diameter FOV ( 4.1 mm in imaging depth). EW: oesophageal wall, LBV2: large blood vessels, TC: trachea, FM: folded mucosa. Reproduced from [142]. (g) FLIM: Not used in Barrett's. (h) MPM: Not used in Barrett's. (i) ERS: The mean in vivo confocal Raman spectra of columnar-lined epithelium, non-dysplastic Barrett's, and high-grade dysplasia acquired from Barrett's patients during clinical endoscopy. Each Raman spectrum is acquired within 0.1-0.5 s. Reproduced from [97]. (j) CARS: Not used in Barrett's. (k) MSI: Not used in Barrett's. 
Angle-resolved low coherence interferometry (a/LCI) also looks at singly-scattered light but probes the angular scattering distribution at a single wavelength, from which measurements of nuclear size and nuclear density can be derived. Using these, a/LCI has been shown to identify dysplasia (including LGD) with 100\% sensitivity and 84\% specificity in vivo (in vivo, 2 centre pilot study, $n=46$ patients, $n=172$ sample positions) [141] and a negative predictive value of $100 \%$. Although a/LCI was originally a point measurement method, the ability to spatially scan to provide 2D maps that co-register with wide-field images has now been demonstrated [147].

There are also phase and polarisation-sensitive endoscopic methods on the horizon that derive contrast from scattering and present wide-field images [148, 149]. While VLE is currently the most advanced method for interrogating microstructure in terms of clinical translation, if their performance remains high in randomised controlled trials, DRS/ESS, LSS and a/LCI have potential to become valuable optical biopsy techniques, and red flag techniques if 2D spatial scanning can be implemented successfully.

\section{B. Interrogating abnormal tissue function and metabolism}

Angiogenesis, the formation of new blood vessels, is a well-known hallmark of cancer [5], and several optical imaging techniques to probe vascular changes have been investigated. Virtual chromoendoscopy has been successful in improving targeted biopsy based on changes in tissue vasculature, but can only interrogate superficial epithelial changes. OCT has shown exciting possibilities for cross-sectional imaging of tissue function, using measurements of blood flow to highlight the vasculature $[150,151]$, although these have yet to be clinically validated. Another method that provides cross-sectional vascular information is photoacoustic endoscopy (PAE), which uses optical excitation of tissue to generate ultrasound [152]. Highly optically absorbing molecules in tissue, such as oxy- and deoxy- haemoglobin, can be resolved at far greater penetration depth than is available from exclusively optical imaging, and distinguished based on their absorption spectra, allowing vascular function to be imaged in addition to vascular structure. Thus, PAE could provide high resolution cross-sectional functional imaging at centimetre depths, allowing visualisation of vascular changes associated with dysplasia.

PAE devices using a similar helical scanning implementation to OCT [153-155] have been applied in rabbit oesophagi [142], but further development is needed to increase radial resolution and acquisition speed, as well as to address challenges with image interpretation (similar to those of OCT) (Translational Characteristic 4). Recent successful studies using photoacoustic imaging in breast cancer diagnosis and other areas [156] suggests that application

of PAE in Barrett's surveillance may yet yield valuable information, potentially in combination with OCT and other advanced methods [154]. 
Another well-established marker of dysplasia is the change in tissue autofluorescence associated with underlying changes in metabolism. When measured by standard AFI endoscopes, these changes can be confounded by surface irregularities and non-uniform illumination. Fortunately, measuring the lifetime of the fluorescence signal, rather than its absolute intensity, avoids these confounding factors [157]. Fluorescence lifetime imaging microscopy (FLIM) is able to map changes in local tissue microenvironment and has shown promise in detection of cancers in ex vivo and in vivo studies [157]. For many years, the clinical translation of FLIM was limited by the size, cost and complexity of the instrumentation and the need for long integration times due to weak signals (Translational Characteristic 2). A 2003 study of point-based fluorescence lifetime measurements found sensitivity and specificity for HGD of less than $60 \%$ using time resolved fluorescence (in vivo, single centre pilot study, $\mathrm{n}=37$ patients, $n=108$ fluorescence decay profiles) [143]. More recently, however, compact diodepumped laser-based excitation sources and time-gated methods have addressed instrumentation limitations [158] meaning wide-field FLIM endoscopes with near-video rate acquisition $(2 \mathrm{~Hz})$ [159-161] are now available. While these have been used in vivo [159, 162] they have yet to be applied to Barrett's surveillance and may yield improved performance in this context.

Complementing wide-field FLIM approaches, multi-photon microscopy (MPM) provides an autofluorescence-based alternative to pCLE. MPM is a scanning, optical sectioning, imaging approach in which fluorescence is spatially delineated using non-linear optical excitation. $E x$ vivo MPM of fresh biopsies can successfully distinguish squamous mucosa, gastric columnar mucosa and intestinal metaplasia (ex vivo, $\mathrm{n}=25$ patients, $\mathrm{n}=35$ biopsies) [144] suggesting that MPM could be used to identify dysplasia. MPM endoscopes are being developed and can incorporate additional features from microscopy such as super-resolution imaging [163]. Although MPM is at a very early stage of development, it holds potential to perform high magnification, depth-sectioned, label-free endomicroscopy as part of Barrett's surveillance.

\section{Interrogating bulk molecular composition}

Changes in bulk molecular composition can be determined using the inelastic scattering spectral 'fingerprint' measured through endoscopic Raman spectroscopy (RS). RS is sensitive to the abundance of molecular bonds, primarily lipid, protein and nucleic acid content in tissue. RS data is typically classified using machine-learning methods that use a training set of spectra where the disease classification is known from histopathology analysis [164].

Historically, the low intensity of Raman signals has been a hurdle for endoscopic RS, resulting in very slow data acquisition (Translational Characteristic 2). Nonetheless, Bergholt et al. recently demonstrated an ERS probe, including a classification algorithm based on a Raman 
library of $>12000$ spectra, that could differentiate between columnar lined epithelium, nondysplastic Barrett's or HGD, in real time $(0.2 \mathrm{sec})$, passing this information to the endoscopist using auditory feedback as the probe was passed across the tissue (in vivo, pilot study, $\mathrm{n}=77$ patients, sensitivity $87.0 \%$, specificity 84.7\%) (Translational Characteristic 4) [97]. Alternatively, coherent anti-Stokes Raman spectroscopy (CARS), which uses multiple photons to probe specific regions of the spectral fingerprint, has been suggested as a way to overcome the low Raman intensity. Several prototype endoscopes have been developed, despite challenges associated with non-linear effects in fibres and the design of miniature optics [165, 166]. Precision remains a challenge for both endoscopic RS and CARS, due to pressure-based signal variation, but prospective randomized multicentre trials are underway [97] and further devices are in development [167]. Raman spectroscopy is thus a promising technique that could provide both point measurements for optical biopsy, and 2D scanning measurements to assess larger areas of tissue for red flag surveillance.

\section{Multimodal methods}

The recent advances in optical techniques highlighted above suggest that contrast mechanisms based on intrinsic optical interactions with tissue have the potential to improve the diagnostic yield of Barrett's surveillance. Naturally, combining several of these contrast mechanisms into a single device could have added benefits, giving access to structural, functional and molecular information simultaneously. For example, a recent pilot study combining intraoperative DRS, RS and fluorescence spectroscopy achieved 100\% sensitivity and 93\% specificity for several cancers (in vivo, $\mathrm{n}=15$ patients) [168] and another study combining fluorescence spectroscopy, DRS and LSS achieved 93\% sensitivity and 100\% specificity for dysplasia in Barrett's oesophagus (in vivo, $\mathrm{n}=16$ patients) [169]. Achieving a successful combination, however, requires careful optical design and often complex instrumentation (Translational Characteristic 2).

One promising route to overcoming this challenge may lie in the use of multispectral imaging (MSI), where the light illuminating the tissue and being detected is split into its component colours, or rapidly modulated. NBI is a simple example of this, where just 2 wavelength bands of illumination are used, and standard WLE another, where just 3 wavelength bands are detected. MSI goes further, recording 10s or 100s of colours at every pixel in an endoscopic image, which can then be processed to spatially resolve reflectance (e.g. NBI), fluorescence (e.g. AFI) or Raman information, depending on the illumination applied and the signal detected. MSI has already shown potential for aiding cancer diagnosis in a range of organ sites, including the oesophagus [10], although its potential has yet to be demonstrated through in vivo trials in Barrett's surveillance. MSI hardware is often bulky and slow (Translational Characteristic 2) 
but optimisation of the MSI hardware, for example using compact spectrally resolved detector arrays (SRDAs) [170], may assist with real-time clinical application in the future.

\section{E. Optical molecular imaging}

Despite the aforementioned disadvantages of exogenous contrast, application of exogenous molecular imaging agents consisting of a targeting probe and fluorescent optical reporter has the potential to allow wide field visualisation of complex biochemical processes involved in both normal physiology and disease with unparalleled specificity. Optical molecular imaging (OMI) has the potential to allow wide-field red flag visualisation of biochemical changes associated with dysplasia. Molecular imaging agents may be administered topically or intravenously. Intravenous administration uses lower concentrations of probe and is able to target entire tumours rather than only the exposed surfaces, but requires long (hours-days) incubation times. Topical application is fast (minutes) and limits bio-distribution of the probe to the organ of interest, limiting toxicity concerns. In Barrett's surveillance, topical application is preferred as it causes minimum interruption to the endoscopy workflow whilst still reaching the surface cells of interest. Near-infrared rather than visible fluorophores are preferred in order to avoid confounding visible autofluorescence background.

Due to the heterogeneity of gene expression in Barrett's, it is likely that multiple molecular imaging agents will be required to reach high sensitivity and specificity. Since each of these requires a lengthy toxicology analysis before in vivo validation can even begin, translation of OMI is slow (Translational Characteristic 1), but the enticing promise of macroscopic imaging of microscopic biochemical changes, and encouraging results in current trials, continues to drive much development (including our work in Chapter 4). 


\subsection{Summary}

Though several advanced endoscopy techniques have met the ASGE performance standards for recommended routine use in Barrett's oesophagus, histological assessment of HD-WLE targeted and random biopsies is still the standard of care. Many emerging techniques achieve some of the aforementioned requirements, but fall short of providing the ideal solution. Continuing advances in hardware and software are allowing endoscopic application of optical contrast mechanisms developed for other indications. It is likely that the next decade will see some of these exciting new contrast mechanisms applied to Barrett's surveillance in new devices for the first time, potentially leading to techniques that provide a long-awaited improvement to the standard of care.

Reviewing the optical techniques presented in this chapter, it can be concluded that an ideal endoscopic surveillance method should perform comprehensive investigation of the oesophagus with high sensitivity and specificity for dysplasia. It should allow for the use of endoscopic tools for marking and biopsy if necessary (Translational Characteristic 2). If exogenous contrast is chosen, it should be topically applied and provide contrast enhancement enough to justify the increased procedure times and costs, which should be minimised (Translational Characteristic 1). Implementation of the technique should be possible with minimal additional training of endoscopist operators and image interpreters (Translational Characteristic 3, Translational Characteristic 4). To achieve widespread deployment in healthcare systems, no significant change to procedure times, expertise or costs should be made; ideally these would be reduced. Though initial trials are facilitated by the device being compatible with the current standard of care (Translational Characteristic 2, Translational Characteristic 6), if possible, availability of endoscopy to patients should be increased, for example by enabling deployment by nonspecialist operators in primary care centres, and physical discomfort with the procedure should be decreased, to improve compliance with surveillance programmes.

From this review of optical techniques, it is evident that optical molecular imaging (OMI) and multispectral imaging (MSI) provide potentially translatable solutions in Barrett's surveillance, satisfying many of the aforementioned criteria: they provide wide-field 2D images (Translational Characteristic 3), allowing red flag surveillance and coregistration with familiar 2D HD-WLE images (Translational Characteristic 6); they are easily implemented in a standard forward facing device architecture (Translational Characteristic 2); and they acquire images that can potentially be processed to generate a simple intensity map, with bright pixels highlighting suspicious regions of dysplasia, allowing easy interpretation without the need for complex classification algorithms (Translational Characteristic 4). Additionally, MSI utilises endogenous contrast (Translational Characteristic 1). 
Over the next three chapters, work translating two novel techniques towards clinical implementation is described. First, the choice of device architecture under which the prototype imaging devices were prepared is explained, including its advantages and limitations. This is important, as it simplified approval of the devices for use in humans and facilitated subsequent deployment in clinical trials. However, this device architecture introduced artefacts into the images, so the remainder of Chapter 3 is concerned with outlining the image correction algorithms developed to remove these. Next, Chapter 4 describes work on OMI. The design and characterisation of an endoscope for NIR fluorescence imaging of a topically applied molecular imaging agent that binds specifically to Barrett's oesophagus and not to dysplasia is described, and the validation of this technique using a range of biological samples is presented. Finally, in Chapter 5, work developing an endoscope for MSI of endogenous tissue contrast is described. This work led to a pilot clinical trial of multispectral endoscopy in Barrett's surveillance. 



\section{FLEXIBLE ENDOSCOPY: DEVICE ARCHITECTURE}

This chapter uses material from two papers by the author: a review paper entitled "Emerging Optical Methods for Endoscopic Barrett's Surveillance”; and an original research paper entitled "Quantitative evaluation of comb-structure removal methods for multispectral fibrescopic imaging" (see publications list).

In order to apply a novel optical imaging technique, a contrast mechanism must be combined with a device architecture (Table 3.1). Here, emerging endoscopic device architectures are described, and their impact on clinical translation is considered. This is followed by a description of the device architecture we chose to develop, the work done to address its limitations, and the advantages this device architecture affords us.

\subsection{Flexible Endoscopic Device Architectures}

At present, clinical endoscopic surveillance of Barrett's oesophagus is performed using a forward-facing trans-oral endoscope architecture (Figure 3.1 (a)), around which standard endoscopic tools, such as biopsy forceps and treatment devices, have been designed. Often these tools access the patient via a hollow working channel in the body of the endoscope. Forwardfacing endoscopes require articulation by the endoscopist to ensure complete surveillance of the tissue. A key consideration in the development and clinical translation of new optical techniques is whether this remains the appropriate device architecture. An obvious approach to advanced endoscopy is simply to integrate the new contrast mechanism into an existing forward-facing 


\section{Table 3.1 Endoscopic device architectures.}

Schematics of each device architecture are shown in Figure $3.1 .^{* *}$ Theoretically most combinations of contrast mechanism and device type are possible. Here we give the contrast mechanisms that are most compatible with the advantages and disadvantages of the device architecture. *** Image type is again dependent on contrast mechanism. Here we give the image types for the most compatible contrast mechanisms for the device architecture.

\begin{tabular}{|c|c|c|c|c|c|c|}
\hline $\begin{array}{l}\text { Device } \\
\text { Architecture }\end{array}$ & $\begin{array}{l}\text { Example } \\
\text { device(s) }\end{array}$ & Field of view & Advantages & Disadvantages & $\begin{array}{l}\text { Most compatible } \\
\text { contrast } \\
\text { mechanisms** }\end{array}$ & $\begin{array}{l}\text { Typical image } \\
\text { types*** }\end{array}$ \\
\hline $\begin{array}{l}\text { Forward } \\
\text { Facing } \\
\text { (Trans-oral) }\end{array}$ & $\begin{array}{l}\text { Standard } \\
\text { commercial } \\
\text { endoscopes } \\
\text { e.g. Olympus, } \\
\text { Pentax, Fuji }\end{array}$ & $\begin{array}{l}\text { Wide (typically } \\
140^{\circ} \text { luminal } \\
\text { view) }\end{array}$ & $\begin{array}{l}\text { Availability and } \\
\text { familiarity; wide variety } \\
\text { of tools for biopsy, } \\
\text { washing, marking; } \\
\text { articulation. }\end{array}$ & $\begin{array}{l}\text { Endoscopist must } \\
\text { articulate to survey entire } \\
\text { surface. }\end{array}$ & $\begin{array}{l}\text { WLE, } \\
\text { Chromoendoscopy, } \\
\text { NBI, eCLE, OMI, } \\
\text { MSI, Polarimetry }\end{array}$ & $\begin{array}{l}\text { En face, } \\
\text { luminal }\end{array}$ \\
\hline $\begin{array}{l}\text { Forward } \\
\text { Facing } \\
\text { (Trans- } \\
\text { nasal) }\end{array}$ & $\begin{array}{l}\text { Standard } \\
\text { commercial } \\
\text { endoscopes } \\
\text { e.g. Olympus, } \\
\text { Pentax, Fuji }\end{array}$ & $\begin{array}{l}\text { Wide (typically } \\
140^{\circ} \text { luminal } \\
\text { view) }\end{array}$ & $\begin{array}{l}\text { Improved patient tolerance } \\
\text { and no sedation required; } \\
\text { articulation; shorter, less } \\
\text { costly procedure. }\end{array}$ & $\begin{array}{l}\text { Endoscopist must } \\
\text { articulate to survey entire } \\
\text { surface; lower quality } \\
\text { image*; narrower working } \\
\text { channel inappropriate for } \\
\text { interventions; poorer } \\
\text { suction and air function } \\
\text { and smaller biopsy } \\
\text { capabilities compared with } \\
\text { trans-nasal endoscopes. } \\
\text { *unsuitable for Barrett's } \\
\text { surveillance }\end{array}$ & WLE, NBI & $\begin{array}{l}\text { En face, } \\
\text { luminal }\end{array}$ \\
\hline $\begin{array}{l}\text { Accessory } \\
\text { Channel } \\
\text { (e.g. Contact } \\
\text { Probe) }\end{array}$ & 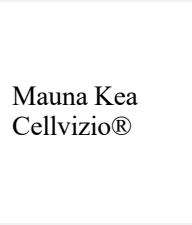 & $\begin{array}{l}\text { Narrow for } \\
\text { contact probe } \\
\text { (10s-100s } \\
\text { microns). }\end{array}$ & $\begin{array}{l}\text { Compatible with insertion } \\
\text { through working channel } \\
\text { of standard endoscopes. }\end{array}$ & $\begin{array}{l}\text { Must be used alongside } \\
\text { standard endoscope for } \\
\text { articulation, washing, } \\
\text { biopsy, marking; contact } \\
\text { with lumen must be } \\
\text { carefully controlled; small } \\
\text { field of view. }\end{array}$ & $\begin{array}{l}\text { pCLE, ERS, } \\
\text { ESS/DRS, a/LCI, } \\
\text { MSI, FLIM, MPM, } \\
\text { PA, Polarimetry }\end{array}$ & $\begin{array}{l}\text { Spectral, en- } \\
\text { face }\end{array}$ \\
\hline $\begin{array}{l}\text { Balloon } \\
\text { Based }\end{array}$ & $\begin{array}{l}\text { NinePoint } \\
\text { NvisionVLE® }\end{array}$ & Volumetric & $\begin{array}{l}\text { Controlled withdrawal; } \\
\text { potential for cautery } \\
\text { marking capability; } \\
\text { compatible with insertion } \\
\text { through working channel } \\
\text { of standard endoscopes; } \\
\text { allows full volumetric } \\
\text { imaging of oesophagus. }\end{array}$ & $\begin{array}{l}\text { No biopsy or washing } \\
\text { capabilities. }\end{array}$ & OCT & Volumetric \\
\hline $\begin{array}{l}\text { Wireless } \\
\text { Capsule }\end{array}$ & $\begin{array}{l}\text { Given Imaging } \\
\text { PillCam } \AA \text { ESO } \\
\text { series }\end{array}$ & $\begin{array}{l}2 \times 169^{\circ} \\
(\mathrm{ESO} 2)\end{array}$ & $\begin{array}{l}\text { No sedation required; can } \\
\text { be implemented in } \\
\text { primary care; potential for } \\
\text { low cost if reusable. }\end{array}$ & $\begin{array}{l}\text { One shot (cannot return to } \\
\text { suspicious lesions); no } \\
\text { biopsy, washing, marking } \\
\text { capabilities; long delay for } \\
\text { capsule to pass ( } 8-10 \\
\text { hours); no control over } \\
\text { motion; contact with } \\
\text { lumen must be controlled. }\end{array}$ & $\begin{array}{l}\text { WLE, NBI, MSI, } \\
\text { Polarimetry }\end{array}$ & $\begin{array}{l}\text { En face, } \\
\text { luminal or } \\
\text { circumferential }\end{array}$ \\
\hline $\begin{array}{l}\text { Tethered } \\
\text { Capsule }\end{array}$ & $\begin{array}{l}\text { No commercial } \\
\text { devices }\end{array}$ & Volumetric & $\begin{array}{l}\text { No sedation required; can } \\
\text { be implemented in } \\
\text { primary care; potential for } \\
\text { low cost if reusable; } \\
\text { controlled withdrawal; } \\
\text { potential for cautery } \\
\text { marking capability; } \\
\text { immediate removal of } \\
\text { capsule; allows full } \\
\text { volumetric imaging of } \\
\text { oesophagus. }\end{array}$ & $\begin{array}{l}\text { No biopsy, washing } \\
\text { capabilities; contact with } \\
\text { lumen must be controlled. }\end{array}$ & OCT & Volumetric \\
\hline Wide Angle & $\begin{array}{l}\text { EndoChoice } \\
\text { Fuse }\left(330^{\circ}\right)\end{array}$ & $\begin{array}{l}\text { Extra wide } \\
\left(>140^{\circ}\right)\end{array}$ & $\begin{array}{l}\text { Familiarity; wide variety } \\
\text { of tools for biopsy, } \\
\text { washing, marking; } \\
\text { articulation; wide field of } \\
\text { view allows viewing of } \\
\text { entire lumen with minimal } \\
\text { articulation. }\end{array}$ & Increased cost. & $\begin{array}{l}\text { WLE, } \\
\text { Chromoendoscopy, } \\
\text { NBI, eCLE, OMI, } \\
\text { MSI, Polarimetry }\end{array}$ & $\begin{array}{l}\text { En face, } \\
\text { circumferential }\end{array}$ \\
\hline
\end{tabular}


(a) Forward Facing

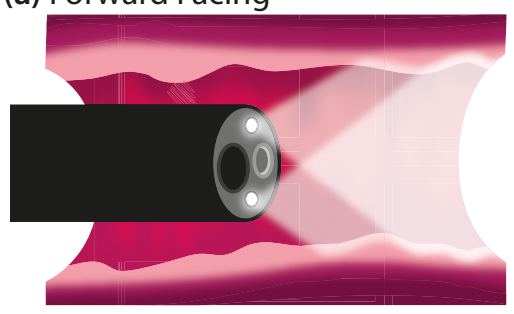

(d) Wireless Capsule

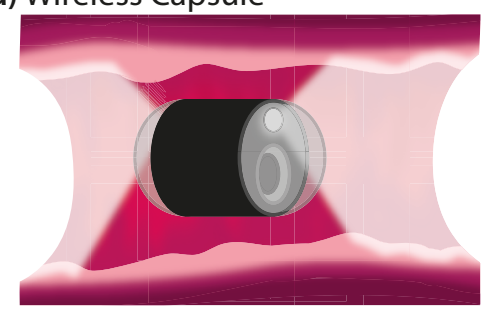

(b) Accessory Channel

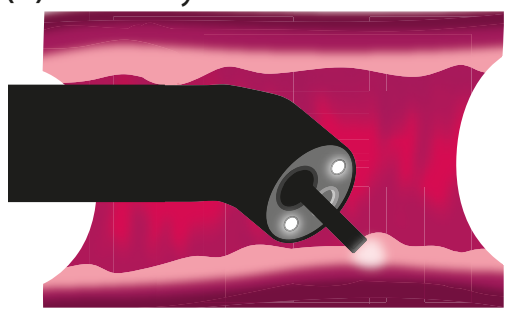

(e) Tethered Capsule

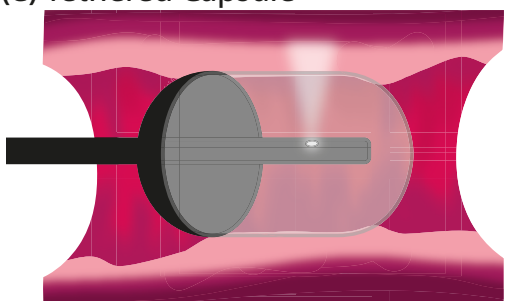

(c) Balloon Based

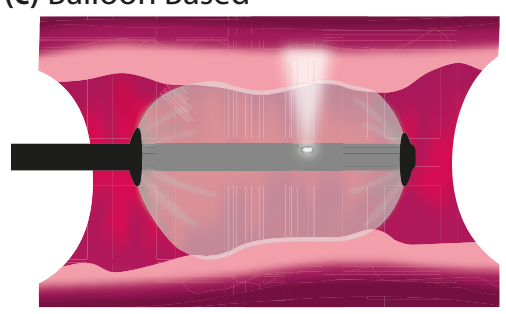

(f) Wide Angle

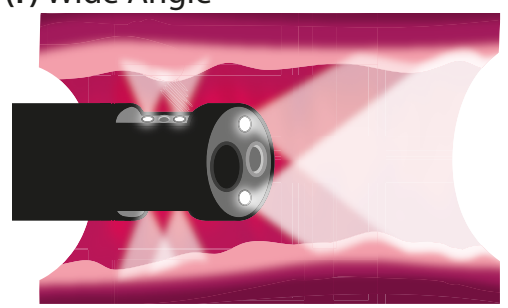

Figure 3.1 Endoscopic device architectures - schematics.

Schematic representations of the endoscopic device architectures listed in Table 3.1.

endoscope, to exploit familiarity of endoscopists with the device and retain access to the usual endoscopic tools. In recent years, research groups and commercial companies have taken a more 'out-of-the-box' approach, developing a host of alternative device architectures that overcome some or all of the limitations of forward-facing endoscopy, namely: low magnification; high procedure cost; the need for specialist operators; and restricted angular field of view (Table 3.1).

Accessory channel endoscopes (Figure 3.1 (b)), are small-diameter probes that can be inserted into standard forward-facing endoscopes through the channel that is normally used to introduce tools. This allows them to be controlled by the standard endoscope; allows their images to be coregistered with standard imaging; and allows them to be implemented without overhaul of the existing equipment and facilities (Translational Characteristic 6). In contrast to forwardfacing trans-oral endoscopes, where the camera is found on the tip of the endoscope, accessory channel endoscopes often relay light to detectors outside the patient, using a fibre bundle consisting of 10,000-50,000 individual glass fibrelets [171].

These probes are often placed in direct contact with oesophageal tissue to perform optical biopsy. Unlike physical biopsy, increasing the number of these optical biopsies does not add significantly to procedure cost, however, the endoscopist must manually control the position of the accessory channel endoscope using the standard endoscope, so the results will still be subject to sampling error. In addition to this, the physiological movements of the oesophagus due to peristalsis and anatomic vicinity to the heart, make stabilisation of probe-tissue contact challenging. In other cases, rather than direct contact, the imaging device employs a balloon 
(Figure 3.1 (c)) that is inflated to ensure a fixed distance between the tissue and the central axis of the imaging hardware [31, 128].

The high procedure cost of forward-facing endoscopy arises from the need for patient sedation in a specialist facility with a skilled endoscopist. Un-sedated trans-nasal endoscopy (UTNE) provides standard endoscopy capabilities (imaging, articulation, insufflation, suction, biopsy) in a slim device that can be used without sedation, as the trans-nasal intubation does not involve contact with the root of the tongue and therefore does not trigger the gagging reflex. Multiple UTNE systems are commercially available, including two disposable devices which make reprocessing feasible outside of a hospital environment [116]. UTNE has been successfully used for imaging Barrett's and dysplasia [172-174]. Recent studies using UTNE to screen for Barrett's have found it to be comparable to standard endoscopy in clinical effectiveness, participation and safety [175] and considerably cheaper, especially if implemented in a mobile unit instead of a hospital [176]. Nonetheless, UTNE image quality is currently insufficient for dysplasia detection.

While UTNE still requires a skilled endoscopist, wireless capsule endoscopes (Figure 3.1 (d)) are single-use, pill-shaped devices that can be administered by a non-specialist operator (Translational Characteristic 3). Originally developed for small bowel imaging [177], wireless capsules have since been developed for the oesophagus [178]. When surveyed, most patients prefer capsule endoscopy to regular endoscopy [179], which may improve adherence to surveillance protocols. Capsule endoscopy has become the gold standard for the small bowel, but studies in the oesophagus have yielded mixed results [180]. Wireless capsules have several significant limitations in the oesophagus [181]: the need for a reclined ingestion protocol to increase the imaging period during swallowing from seconds to minutes [180]; difficulty in identifying the capsule location for a given image; and the inability to take biopsies during the procedure.

Tethered capsule endoscopes (Figure 3.1 (e)) retain the benefits of wireless capsules while addressing several of their limitations in the oesophagus by using a cord to control the capsule's position [129]. The tethered capsule is swallowed by the patient in an upright position, then imaging is performed while it is pulled back up from the stomach. The tethered architecture eliminates the risk of capsule retention and opens up the possibility of capsule re-use, which could lower per-procedure costs [182]. A tethered capsule architecture for Barrett's surveillance is currently in clinical trials [68].

To survey the entire oesophagus during endoscopy, careful articulation of the endoscope is required to bring the entire luminal surface within the $140^{\circ}$ forward-facing field of view. 
Increased inspection time has been associated with an increased HGD and cancer detection rate [183], but it is unclear whether this is due to additional time inspecting each suspicious site, or additional time articulating the scope to survey the lumen more exhaustively. If the latter is the case, wide angle endoscopes (Figure 3.1 (f)) [184] with a $330^{\circ}$ field of view may allow improved detection rates. Indeed, this has been successfully demonstrated in the colon, decreasing adenoma miss rate from $41 \%$ to $7 \%$ [185] compared to standard forward-facing devices, although this was not confirmed in a recent randomised study [186]. In the oesophagus, where the surface is smoother with less obscured crevices, wide angle devices may not confer such advantages, but since none have yet been implemented, their potential benefits are unclear. Multiple detectors have also been used to create stereoscopic devices [187], which allow 3D reconstruction of surfaces. It remains unclear whether this innovation will be useful for upper endoscopy, where tissue is relatively smooth.

\subsection{The PolyScope Accessory Channel Endoscope}

Having reviewed the available device architectures, it was considered that in order to achieve clinical translation within the 4 year timeframe of the work presented in this thesis, an existing accessory channel endoscope device architecture would be most suitable. The PolyScope disposable endoscope (PolyDiagnost, Germany) was chosen (Figure 3.2). Briefly, an imaging fibre bundle consisting of 10,000 individual fibrelets (PolyDiagnost) is threaded inside a disposable catheter that protects the distal tip of the imaging fibre bundle from direct patient contact with a glass cover plane (PolyDiagnost). The catheter also contains a single fibre light guide for illumination and a $1.2 \mathrm{~mm}$ working channel. A range of optical fibre bundles and catheter lengths are available. The PolyScope has several advantages (Figure 3.3 (a)):

- The PolyScope is CE marked for endoscopic retrograde cholangiopancreatography (ERCP) when passed through the accessory channel of a standard upper gastrointestinal endoscope that passes through the digestive system to the biliary duct. This CE mark means this device is fit for intended purpose, meets legislation relating to safety and can be marketed within the EU, facilitating local approval for safe use in the oesophagus (Translational Characteristic 2).

- Lack of direct patient contact removes the need for sterilisation of the imaging fibre between procedures, prolonging the lifetime of this expensive component of the system. Furthermore, since the only part of the system in contact with the patient is an unmodified disposable catheter, safety approval is further facilitated (Translational Characteristic 2). 
- The catheter has a maximum diameter of $3.0 \mathrm{~mm}$, allowing it to be threaded into the $3.7 \mathrm{~mm}$ working channel of a commercial standard of care endoscope. This allows; articulation of the device using the standard scope; direct comparison to HD-WLE; and ease of intergation into clinical workflow (Translational Characteristic 6).

- The PolyScope allows light to be coupled into and out of the oesophagus, avoiding challenges with miniaturisation of optics, and allowing great flexibility in design of the illumination and detection paths.

However, the use of the PolyScope poses several challenges (Figure 3.3 (b)):

- The field of view (FOV) of the PolyScope is limited. We have two PolyScope fibre bundles, one with a $70^{\circ}$ FOV (PD-PS-0093) and another with a $120^{\circ}$ FOV (PD-PS-0095), both much less than the typical $140^{\circ}$ FOV of standard forward-facing endoscopes. Furthermore, the $120^{\circ}$ PolyScope FOV is cropped due to the catheter tip obscuring the edge of the FOV. Despite efforts to remove this, including PolyDiagnost manufacturing a custom $100^{\circ}$ scope, this artefact persists, forcing us to accept a limited FOV for our proof of concept device.

- Coupling light into a single $500 \mu \mathrm{m}$ fibre such that the resulting illumination power at the tip is sufficient for imaging presents a challenge. Initially, a crude butt coupling method interfaced by a PolyDiagnost coupler was used, and illumination power was increased to account for the poor transmission (Chapter 4.4.2). Later, a custom illumination system, including coupling adaptor, was designed and manufactured (Chapter 5.4.2).

- The main challenge in working with the PolyScope arises from the fibre bundle. Despite allowing convenient coupling of light into and out of the oesophagus in a narrow accessory channel device, fiber bundles are associated with low resolution and a honeycomb like image artefact arising from the opaque cladding between the fiberlets. The resulting image is of low resolution and is displeasing to endoscopists who are accustomed to high resolution HD-WLE images. Since the aim of this work was to develop red flag techniques, which do not rely on high resolution details of the image, but rather on macroscopic reflectance/fluorescence features highlighting suspicious regions, the limited resolution was accepted as a compromose in light of the key advantages previously identified. However, the honeycomb artefact required careful consideration, and will be the subject of the remainder of this chapter. 


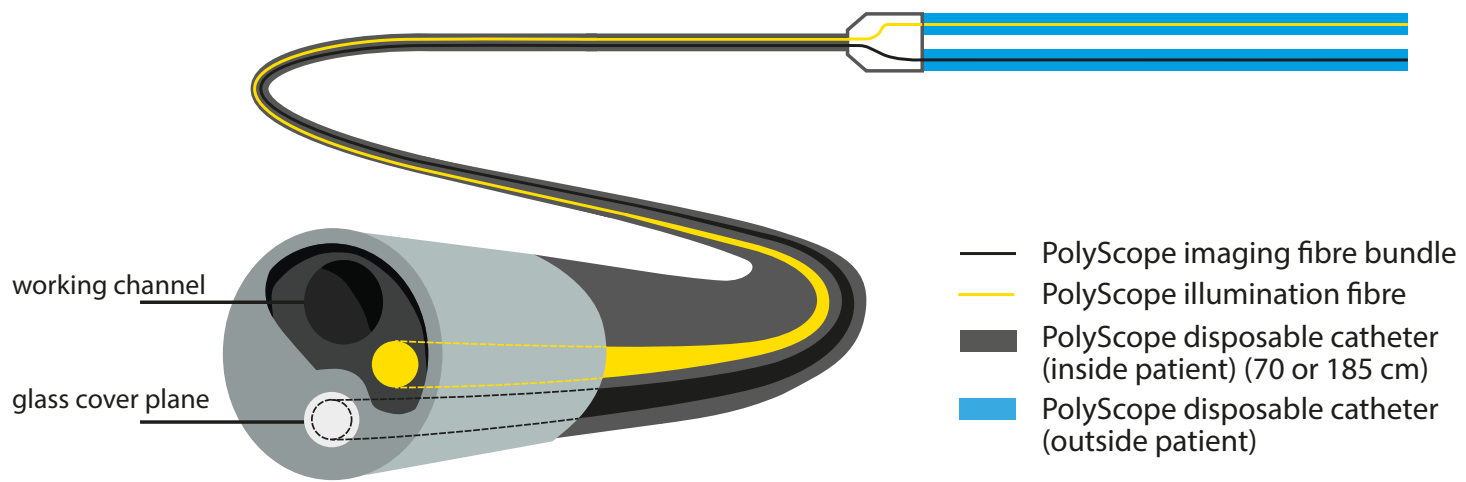

Figure 3.2 The PolyScope accessory channel endoscope.

The PolyScope (PolyDiagnost, Germany) is an accessory channel endoscope CE marked for endoscopic retrograde cholangiopancreatography (ERCP) when passed through the accessory channel of a standard upper gastrointestinal endoscope that passes through the digestive system to the biliary duct. An imaging fibre bundle consisting of 10,000 individual fibrelets is threaded inside a disposable catheter that protects the distal tip of the imaging fibre bundle from direct patient contact with a glass cover plane. The catheter also contains a single fibre light guide for illumination and a $1.2 \mathrm{~mm}$ working channel. Catheter lengths of 70 and $185 \mathrm{~cm}$ are used in this thesis.

(a) PolyScope Advantages

(b) PolyScope Challenges

Solutions
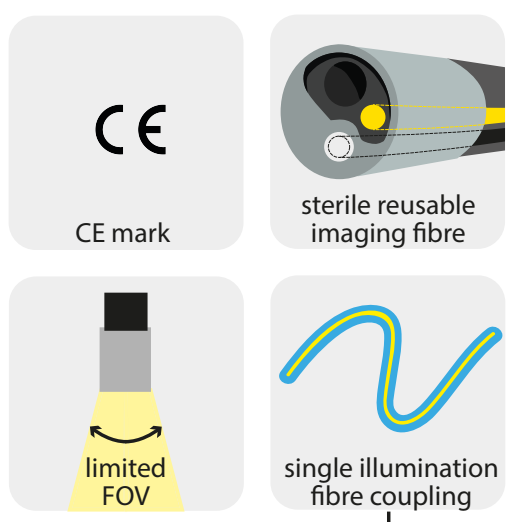

$\downarrow$

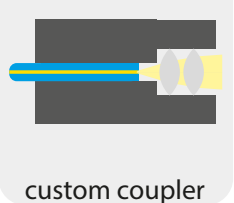

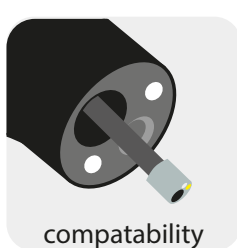
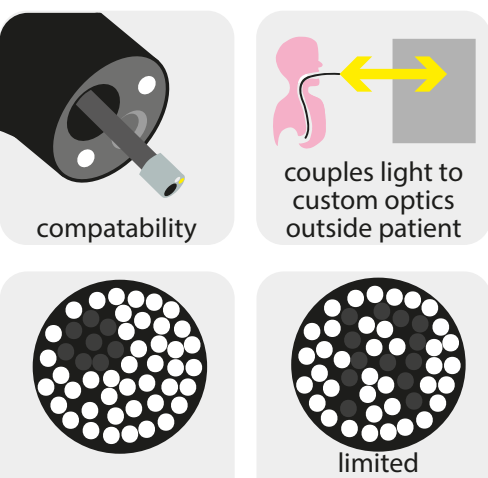

comb artefact

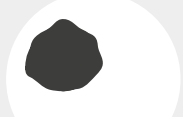

comb removal methods

\section{Figure 3.3 Advantages and Challenges of the PolyScope disposable endoscope.}

(a) The PolyScope system has several advantages: it has a CE mark; the imaging fibre bundle remains sterile and is thus reusable; the PolyScope can be deployed via the working channel of commercial endoscopes; and the PolyScope allows us to couple light to custom optics outside the patient, allowing great flexibility with device design. (b) There are also some challenges. Limited field of view and resolution were accepted as a trade-off for the aforementioned advantages. The single illumination fibre requires careful coupling to ensure adequate light intensity inside the patient, hence the need for a custom coupler. The comb artefact must be removed to achieve images suitable for clinical use by an endoscopist. 


\subsection{Comb Correction Methods}

The term 'honeycomb' is generally reserved for the regular hexagonal artefact introduced by a regularly packed fibre bundle, whereas the PolyScope fibre bundle results in an irregular artefact referred to simply as a 'comb' structure [188] (Figure 3.4). When removing this comb artefact in colour imaging, we must pay careful attention to the impact of the underlying colour filter array (CFA), spectral filters deposited on the pixels of the sensor. Conventional colour cameras have a $2 \times 2$ Bayer CFA super-pixel of red, green and blue filters (Figure 3.5 (a)). Spectrally resolved detector arrays (SRDAs) generalise the conventional Bayer CFA with $3 \times$ $3,4 \times 4$ or even $5 \times 5$ CFA super-pixels enabling multispectral imaging (Figure 3.5 (b)) (Chapter 2.2.3D) $[189,190]$. This introduces a further complication to the image data. A software correction process therefore requires both removal of the comb artefact ("decombing") and separation of the spectral bands ("demosaicking") maintaining both image quality and spectral fidelity [191].

Decombing methods have been extensively explored in the literature and can be readily applied to smooth the appearance of monochrome image data. Winter et al. developed several Fourierbased filtering techniques and compared these to median and Gaussian filters in terms of smoothness (image variance-based) and detail preservation (resolution-based) [188]. They also developed an alternative algorithm to accurately locate and interpolate between fibrelet centres $[192,193]$, testing a number of interpolation strategies developed in other fields. Standard linear interpolation was found to be most suitable when low processing times are required, as in videorate imaging [194]. Later work by Lee et al., Regeling et al. and Han et al. further considered different sizes and shapes of Fourier-based filters for decombing, although did not compare these to the other correction strategies $[191,195,196]$.

None of these prior studies considered the impact of decombing on signal preservation and as they all used monochrome cameras, they did not address the challenge of combined decombing and demosaicking encountered in colour and more generally, in multispectral imaging. A recent paper by Wang et al. did assess an interpolation strategy in the context of a multispectral fibrescope with an SRDA, but only with respect to the accuracy of the spectral reconstruction and without comparison to other methods [197].

To address these limitations, a thorough comparison of decombing techniques combined with demosaicking is presented in this chapter. Performance is assessed with respect to five metrics defined for imaging applications: processing time, resolution, smoothness, signal and accuracy 


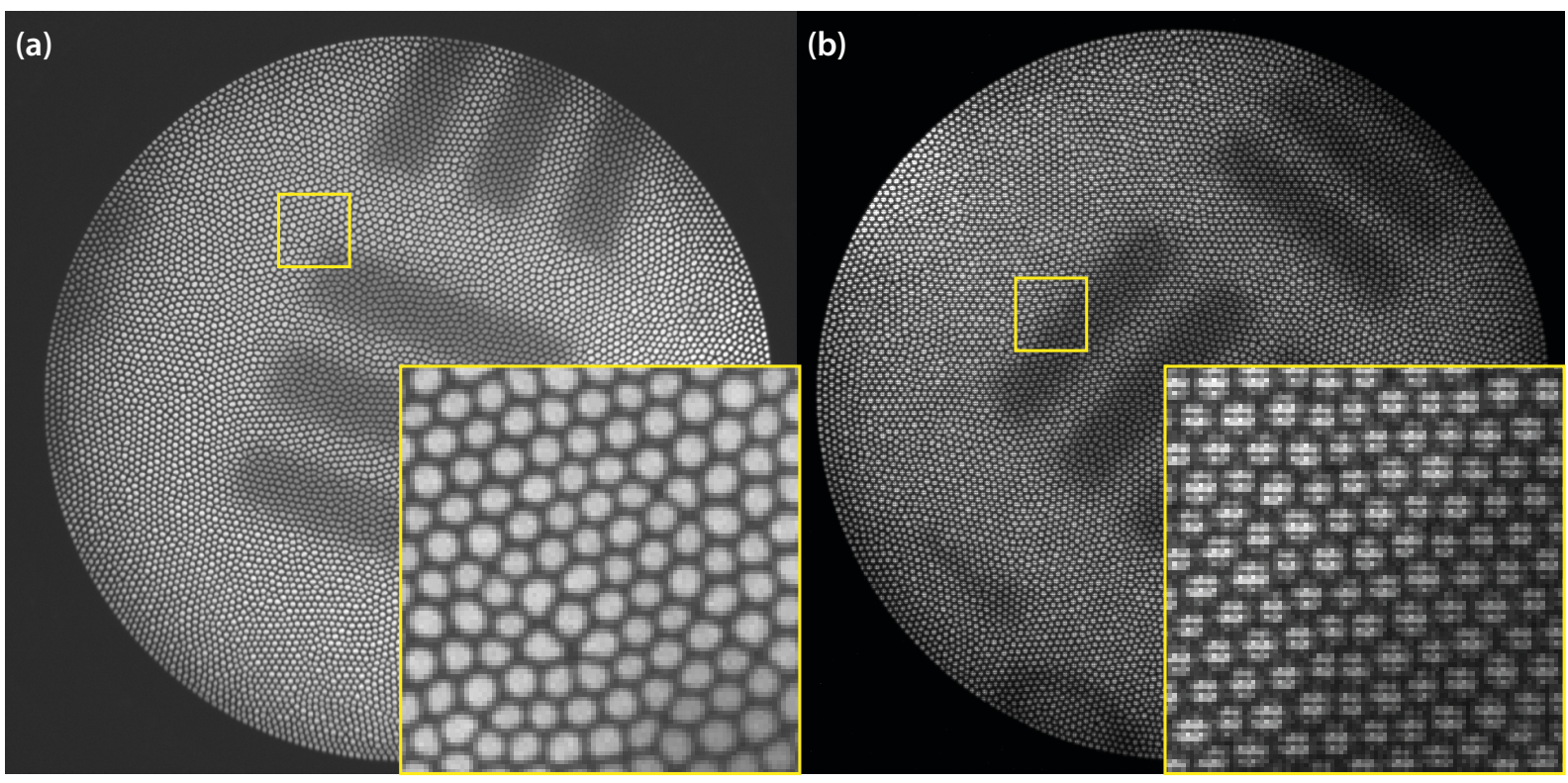

Figure 3.4 Example image with visible comb artefact and mosaic.

(a) Example comb structure from the monochrome fibrescope. Raw image of a USAF test target taken with a monochrome fibrescope (cropped to $\sim 980 \times 980$ ). Inset: Zoom of the comb structure showing irregular arrangement and shape of fibrelets. (b) Example comb structure and colour filter array (CFA) mosaic pattern from the multispectral fibrescope. Raw image of a USAF test target taken with a multispectral fibrescope (cropped to $\sim 980 \times 950$ ). Inset: Zoom of the comb structure showing the superimposed mosaic due to the pixel-by-pixel transmission variations of the $3 \times 3$ mosaic of filters deposited pixel-wise on the sensor.

(a) Conventional colour cameras

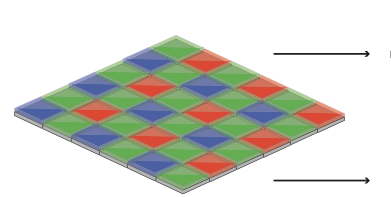

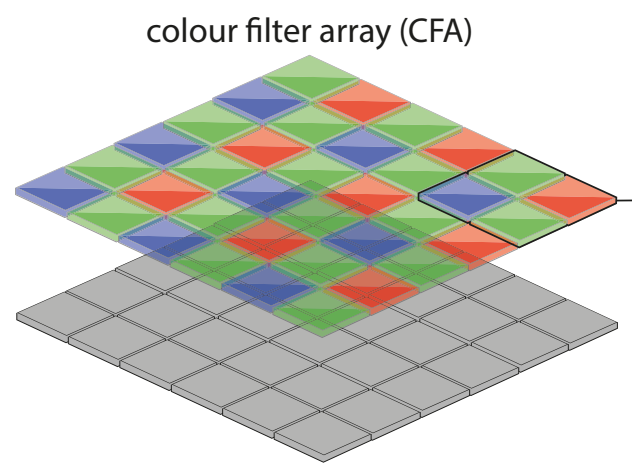

underlying monochrome sensor (b) Example CFA super-pixels

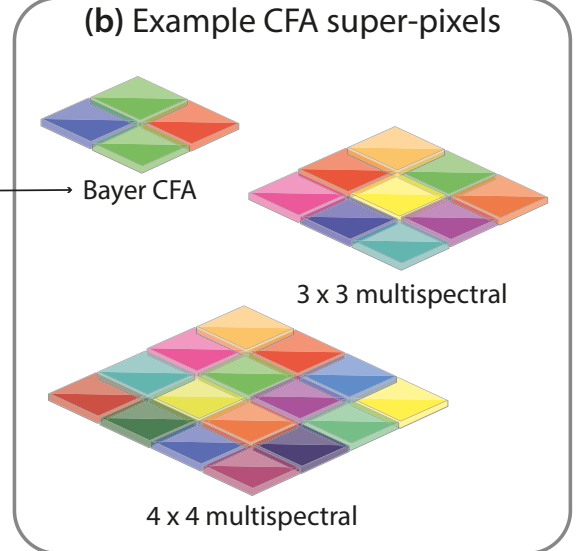

Figure 3.5 Schematic of spectrally resolved detector arrays (SRDAs) and colour filter arrays (CFAs).

(a) Conventional colour imaging is achieved using a colour filter array (CFA), a mosaic of red, green and blue (RGB) colour filters, arranged over a monochrome sensor. (b) The conventional RGB CFA is a Bayer filter. Spectrally resolved detector arrays (SRDAs) generalize the conventional Bayer CFA with $3 \times 3,4$ $\times 4$ or even $5 \times 5$ CFA super-pixels enabling multispectral imaging. 
of spectral reconstruction. Filtering methods tested are median, Gaussian and Fourier filtering, compared against interpolation and physical blurring. Initially, performance was evaluated in simulation. Then, an experimental system was designed and used to acquire monochrome and multispectral images, which were used to evaluate performance on real data. Finally, graphs from which the optimum method can be chosen based on the relative importance of each performance metric for a given application were created.

\subsubsection{Experimental System}

The experimental system was based around the PolyScope (PolyDiagnost, Germany). A narrow band ultra-high power LED (UHP-T-LED-635-EP, Prizmatix, Israel) was coupled into the PolyScope illumination channel using an achromatic doublet lens (AC254-030-A, Thorlabs, Germany) housed inside a custom coupler with a smooth bore for the PolyScope illumination fibre tip (Figure 3.6 (a)). For reflective samples, external illumination was used to reduce specular reflections (Figure 3.6 (b)). The detection pathway consisted of an objective lens (NA $=0.5$, UPLFLN20x, Olympus, Japan) and an achromatic doublet lens ( $f=100 \mathrm{~mm}$, ACA254100-A, Thorlabs, Germany), which focused light from the 10,000-fibrelet bundle onto a monochrome CMOS sensor (GS3-U3-41C6M-C, Point Grey, Canada) or a compact SRDA (CMS-V, SILIOS, France) (square pixel sizes $5.5 \mu \mathrm{m}$ and $5.3 \mu \mathrm{m}$ respectively). The SRDA consists of 9 spectral filters ( 8 narrow bands; average FWHM $30 \mathrm{~nm}$; centre wavelengths 553 , $587,629,665,714,749,791,829 \mathrm{~nm} ; 1$ broad band; 500-850 nm), deposited as a $3 \times 3$ superpixel across the CMOS sensor. The resulting images using a monochrome camera and multispectral SRDA are shown in Figure 3.4.

\subsubsection{Simulated Images}

We simulated monochrome images as outlined in Figure 3.7 using Matlab® (MathWorks, USA). Briefly, an experimental monochrome comb image was binarised to yield an 'ideal' comb mask; the mask was then magnified by different factors, $M$, relative to the original in order to investigate the effect of using different fibrelet diameters. 'Ideal' test images were generated as follows: a series of USAF targets to test resolution, an image with uniform intensity to test smoothness and an image with a region of high intensity to test signal. The ideal binary comb masks and the test images were Gaussian blurred to represent imperfections in the fibrelets and imperfections in the test targets respectively. Gaussian noise was added to the ideal test images. 


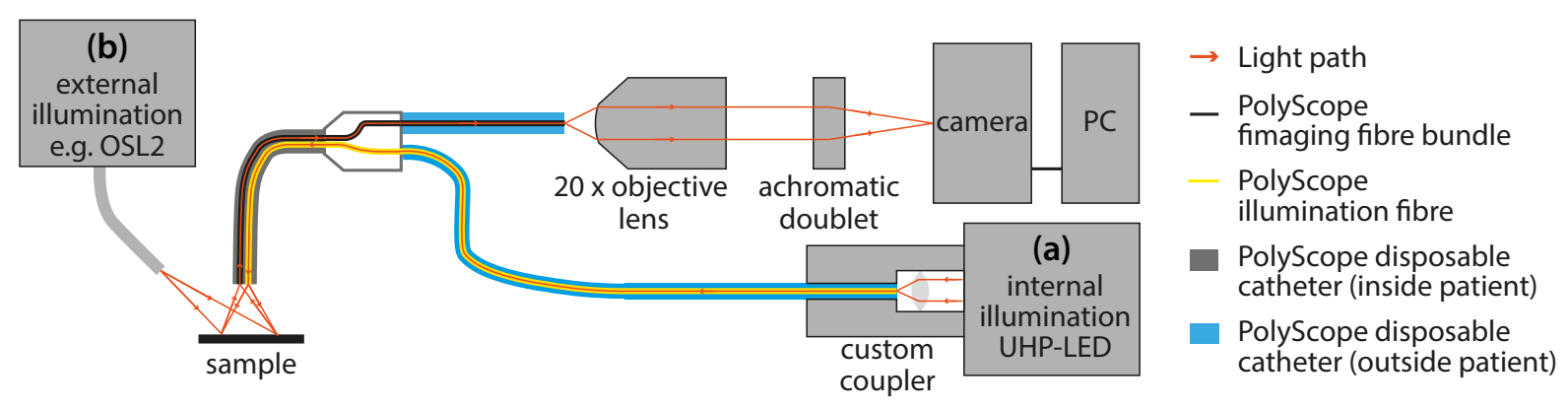

Figure 3.6 Schematic of the fibrescope.

The system is based around the PolyScope accessory channel endoscope (PolyDiagnost, Germany). Illumination is provided either (a) internally, via the PolyScope illumination fibre, with an ultra-high powered LED (UHP-T-LED-635-EP, Prizmatix, Israel) or (b) externally, with a broadband halogen light source (OSL2, Thorlabs, Germany) to reduce specular reflections. Light from the PolyScope 10,000 fibre imaging bundle is focused onto either a monochrome camera (Grasshopper 3, Point Grey, Canada) or an SRDA based multispectral camera (CMS-V, SILIOS, France).

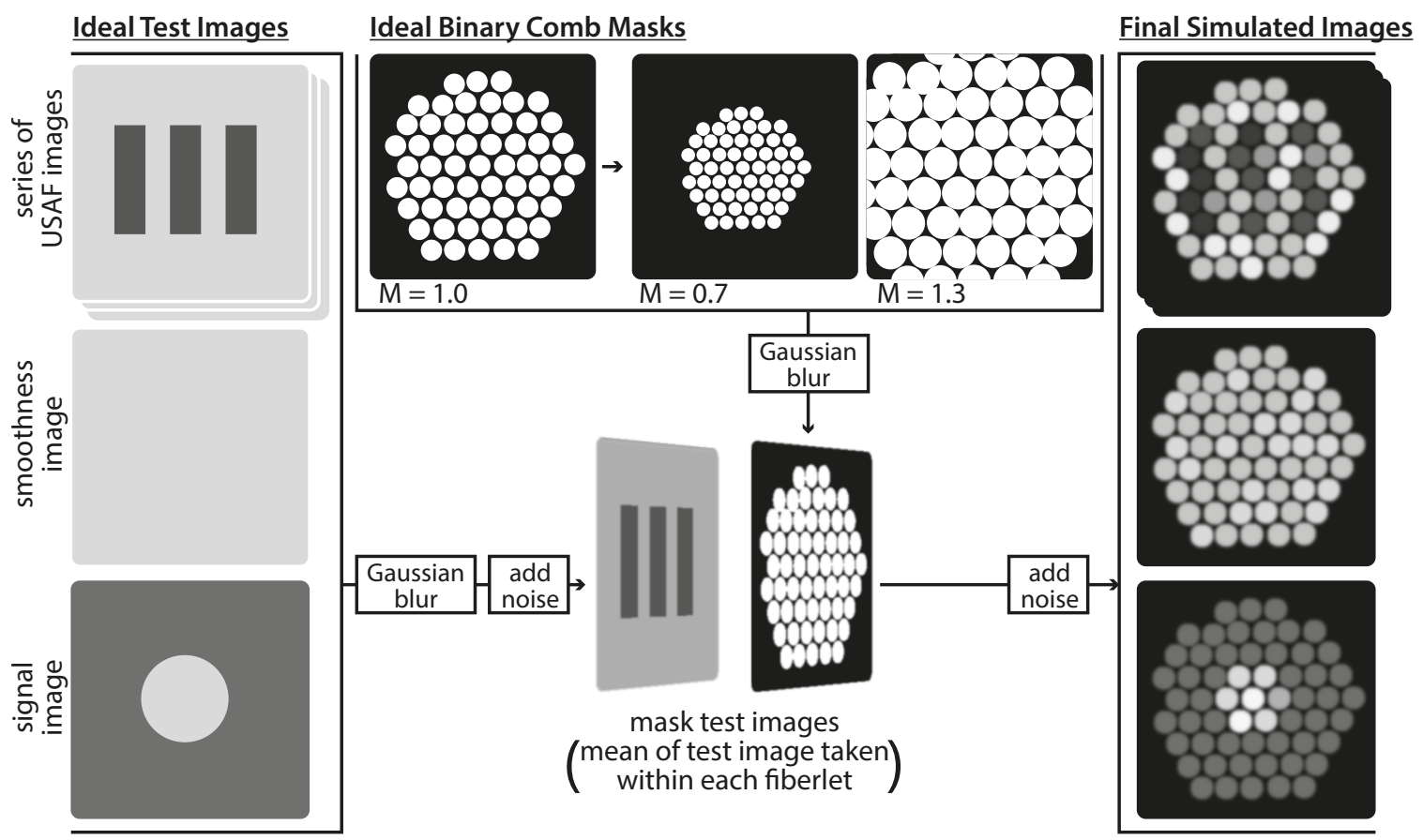

Figure 3.7 Schematic of the image simulation process.

An experimental comb image was binarised to yield an 'ideal' binary comb mask. This was enlarged by a factor, M, compared to the original to create stretched binary comb masks. 'Ideal' test images were generated; a series of USAF targets to test resolution, an image with uniform intensity to test smoothness and an image with a region of high intensity to test signal. The ideal binary comb masks and the test images were blurred to represent imperfections in the fibrelets and imperfections in the test targets respectively. Noise was added to the ideal test images. Next, each of the comb masks was used to mask each test image as follows. The comb mask was split into individual fibrelet masks and for each fibrelet the mean of the test image in the region where the intensity of the fibrelet mask was $>0.1$ was taken, then multiplied by the fibrelet mask, and added to the final image. Noise was added to reach the final simulated images. 
Next, each of the comb masks, $C(x, y)$, was used to mask each test image as follows:

1. The comb mask, $C(x, y)$, was split into individual fibrelet masks, $C_{i}(x, y)$.

2. Each individual fibrelet mask was binarised to generate binarised individual comb masks:

$$
C_{i}^{B}(x, y)= \begin{cases}1, & C_{i}(x, y)>0.1 \\ 0, & C_{i}(x, y) \leq 0.1\end{cases}
$$

3. In the region defined by $C_{i}^{B}(x, y)$, the mean of the test image, $T(x, y)$, was taken:

$$
M_{i}=\sum_{x, y} T(x, y) C_{i}^{B}(x, y) / \sum_{x, y} C_{i}^{B}(x, y)
$$

4. This was multiplied by the individual fibrelet masks, $C_{i}(x, y)$, to generate the simulated image, $I^{\text {sim }}$ :

$$
I^{\operatorname{sim}}(x, y)=\sum_{i} C_{i}(x, y) M_{i}
$$

Finally, Gaussian noise was added to reach the final simulated images.

\subsubsection{Monochrome Image Comb Correction}

All image analysis was performed in Matlab® (MathWorks, USA). Five different decombing methods were applied directly to simulated and experimentally captured monochrome images. For all methods, the amount of filtering is defined by a dimensionless characteristic filter size $r$, which is varied. Gaussian blur was achieved using the Matlab function 'imgaussfilt' to filter the images with a 2D Gaussian smoothing kernel with a standard deviation of $r(1<r<50$ pixels). Median filtering was achieved using the Matlab function 'medfilt2' to filter images by taking the median value of each $2 r$-by-2r-pixel region $(1<r<50$ pixels $)$. Fourier filtering makes use of the Matlab fast Fourier transform function, 'fft2'. The Fourier transform image is cropped to remove any frequencies above $f_{o}$, the cut-off frequency, and inverse Fourier transformed, using 'ifft2', to obtain the corrected image. The cut-off frequency was defined as:

$$
f_{o}=\frac{1}{2 r}
$$

where $r$ is the characteristic filter size input $(1<r<50$ pixels $)$ and corresponds to the smallest resolvable feature size in the inverse Fourier transformed image according to Nyquist's theorem. 
Interpolation relies on knowing the location of each individual fibrelet in the image, for which we used a centre finding algorithm based on the work of Elter et al. [192]:

i. Acquire a bright field calibration image, $I$, of a white reflectance target.

ii. Candidate points are selected based on their brightness in relation to their local neighbourhood. Around each pixel $(x, y)$ neighbourhood is defined as:

$$
N=I(x-d: x+d, y-d: y+d)
$$

where the size of the neighbourhood, $D$, can be defined as:

$$
D=2 d+1
$$

where $d$ is specified by the user such that $D$ is roughly the diameter of a single fibrelet. Given a minimal intensity difference $I_{\min }$, which is specified by the user as the expected minimum intensity difference between fibrelets and cladding, a pixel $(x, y)$ is considered as a candidate centre point if:

$$
\max (N)-\min (N)>I_{\min }
$$

iii. For each candidate centre point, $\left(x_{c}, y_{c}\right)$, a score, $s_{c}$, is calculated indicating how well a 2D quasi-Gaussian surface fits the neighbourhood around this point:

$$
s_{c}=\sum_{N}(G(x, y)-I(x, y))^{2}
$$

where $G$ is a defined as:

$$
G=H e^{-l^{4} / 2 D^{2}}
$$

with $l$ the distance from the candidate point and $H=I\left(x_{c}, y_{c}\right)$.

iv. Order candidate centre points $\left(x_{c}, y_{c}\right)$ by ascending score $s_{c}$.

v. Starting from the highest ranked centre (lowest score), sequentially place each candidate fibre centre $\left(x_{c}, y_{c}\right)$ onto a centre map if and only if the candidate centre is a minimum distance of one fibre diameter from all centres $\left(x_{m}, y_{m}\right)$ already in the map:

$$
\sqrt{\left(x_{c}-x_{m}\right)^{2}+\left(y_{c}-y_{m}\right)^{2}}>D, \quad \forall m
$$

Fibre centres are added for as long as this criterion is satisfied until all candidates have been added to the map or rejected. 
Decombing is then achieved using bilinear interpolation of the pixel values recorded at the fibre centres, $I\left(x_{m}, y_{m}\right)$.

As a reference gold standard, we also performed physical blurring of our image by experimentally defocusing the image of the fiberscope face. We displaced the fiberscope along the optical axis by $5 \mu \mathrm{m}$ in $0.5 \mu \mathrm{m}$ steps in both directions and recorded images. In order to plot physical blurring alongside the other metrics, the dimensionless characteristic filter size, $r$, is arbitrarily defined as:

$$
r=\left|\frac{\text { displacement }}{0.5 \mu \mathrm{m}}\right|
$$

\subsubsection{Multispectral Image Comb Correction}

Multispectral images consist of a mosaic of spectral information due to the filter deposition pattern of the CFA. In a process known as demosaicking (Figure 3.8 (a)), the final colour image is reconstructed by splitting the raw camera output into 9 incomplete mosaic pattern images that are subsequently interpolated. Demosaicking must occur prior to decombing with Gaussian, median and Fourier filtering, as mixing information from adjacent pixels on the raw image would corrupt the recorded spectral information. Gaussian, median and Fourier filtering were applied in the same way as for monochrome images (Section 3.3.3). When physical blurring was applied to the image at the point of capture, light spreads from each fibrelet, decombing the image prior to its passage through the CFA, so following demosaicking, no further filtering takes place. Interpolation between fibrelet centres must occur in parallel to demosaicking (Figure 3.8 (b)). This was implemented as follows:

i. Acquire a bright field calibration image, $I(x, y)$, with broadband illumination to ensure a signal is recorded in all spectral bands.

ii. Apply a Gaussian blur to the bright field calibration image in order to smooth out the mosaic pattern due to the CFA.

iii. Find the centres of the fibrelets, $\left(x_{m}, y_{m}\right)$, using steps ii-vi of the centre finding algorithm outlined in Section 3.3.3.

iv. At each fibrelet centre point $\left(x_{m}, y_{m}\right)$, we need to know the image intensity in each spectral band $k, I^{k}\left(x_{m}, y_{m}\right)$. The centre point corresponds to a pixel with one spectral filter, giving the intensity in one of the spectral bands. For the other spectral bands, the intensity at the centre point is assumed to be the same as the intensity at the nearest neighbour with the correct spectral filter. The filter deposition pattern on the sensor, $P(x, y)$, is known: 


$$
P(x, y)=\left\{\begin{array}{c}
1 \\
2 \\
\vdots
\end{array}\right.
$$

So, the image intensity, $I^{k}\left(x_{m}, y_{\mathrm{m}}\right)$, can be defined as:

$$
I^{k}\left(x_{m}, y_{\mathrm{m}}\right)=I\left(x_{N N}, y_{N N}\right)
$$

where $\left(x_{N N}, y_{N N}\right)$ is the nearest neighbour pixel with the spectral filter $k$ :

$$
P\left(x_{N N}, y_{N N}\right)=k
$$

This process should result in image data for all spectral bands and at all centre locations:

$$
I^{k}\left(x_{m}, y_{m}\right) \forall k, m
$$

This is generalisable to any size mosaic as long as the ratio, $T$, between the super-pixel size and the fibrelet size on the sensor, obeys the criterion:

$$
T=\frac{L}{D}<1
$$

where $L$ is the size of a super pixel and $D$ is the diameter of the fibrelets on the sensor in pixels. Though not used here, even super pixels (e.g. $2 \times 2,4 \times 4)$ can result in some image points having two equally distant nearest neighbours in some spectral bands, so an appropriate randomized selection or average of these would need to be taken.

Finally, using bilinear interpolation of the pixel values at fibre centres within each spectral band, $I^{k}\left(x_{m}, y_{m}\right)$, a comb free image for each spectral band, $I^{k}(x, y)$, is constructed.

\subsubsection{Performance Metrics}

In order to determine the performance of comb removal, 4 performance metrics for monochrome imaging and 5 performance metrics for multispectral imaging were assessed.

\section{A. Resolution}

Resolution was determined by capturing images of a 1951 USAF resolution test target (\#53714, Edmund Optics, USA) externally illuminated with a broadband halogen light source (OSL2, Thorlabs, Germany) to reduce specular reflections. The Michelson contrast, $C^{M}$, was calculated for each element:

$$
C^{M}=\frac{I_{\max }-I_{\min }}{I_{\max }+I_{\min }}
$$




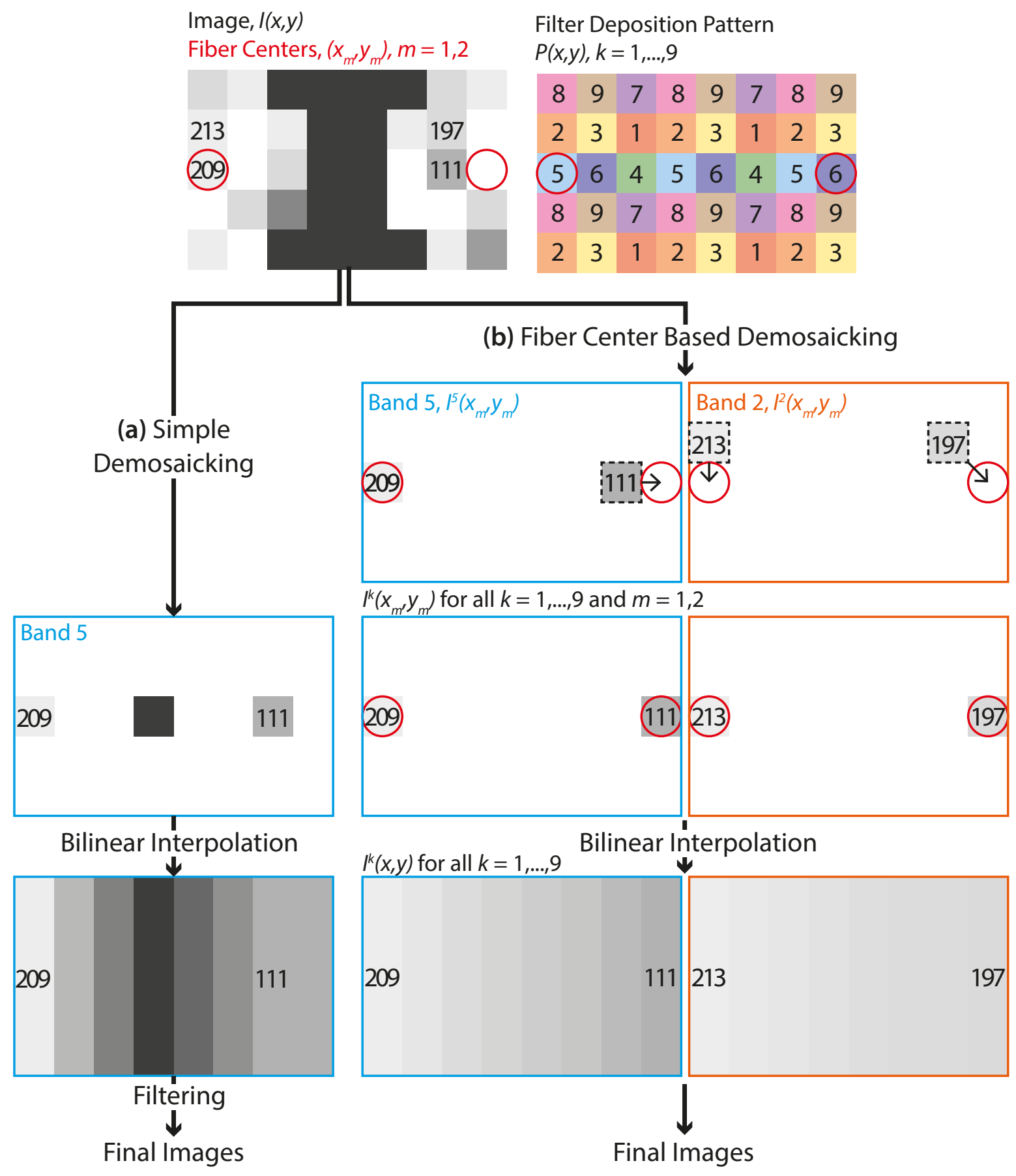

Figure 3.8 Schematic of multispectral demosaicking and comb removal algorithms.

An example image is shown with two fibrelet centres. The corresponding filter deposition pattern for a 9band colour filter array is shown to its right. (a) Simple demosaicking according to the filter deposition pattern is shown for band 5. Following this, bilinear interpolation is used to fill out the image. Finally, filtering of each image occurs, with either a Gaussian, median, or Fourier filter applied, or no filter in the case where physical blurring was applied to the image at the point of capture. (b) Fibre centre based demosaicking is shown for bands 5 and 2. Briefly, at each fibre centre location, the intensity in each band is determined by taking the intensity at the nearest neighbouring pixel with the corresponding band filter. This is followed by interpolation between fibre centres, which can be implemented with a single look up table, since in every band and every image we interpolate between the same centre points. 
where $I_{\max }$ and $I_{\min }$ are the maximum and minimum image intensities in each USAF element respectively.

The resolution, $R$, was determined as the line width when Michelson contrast dropped below $5 \%$ by fitting a smoothing spline to the Michelson contrast versus the line spacing. A contrast threshold of $1 \%$ has previously been reported to be applicable across a wide range of targets and conditions [198], but a contrast threshold of 5\% was chosen to avoid effects arising from noise at very low contrast. A condition requiring data points with contrast $>1 \%$ at $>3$ distinct line spacings was also implemented to ensure there were a reasonable number of non-noise data points to fit a spline. If this condition was not met, a spline was not fitted and the resolution was not defined. For multispectral imaging, the resolution is taken as the average of the resolution determined separately for each of the 9 spectral bands. The resolution score [188], $S_{\text {res }}$, was defined as:

$$
S_{\text {res }}=1-\left(\frac{R-R_{\min }}{R_{\max }-R_{\min }}\right)
$$

where $R_{\max }$ and $R_{\min }$ are the maximum and minimum resolutions calculated across all correction methods, defined such that scores of 1 and 0 represent the best and worst resolution achieved respectively.

\section{B. Smoothness}

For monochrome imaging, smoothness was determined by using images of white areas of a 1951 USAF resolution test target (\#53-714, Edmund Optics, USA) illuminated externally with a broadband halogen light source (OSL2, Thorlabs, Germany) to reduce specular reflections. For multispectral imaging, smoothness was determined using images of a white reflecting target (paper) illuminated with a narrow band LED (UHP-T-LED-635-EP, Prizmatix, Israel) coupled to the PolyScope illumination fibre.

The spatial standard deviation of the supposedly uniform image was calculated. The average of this across 18 images was taken for multispectral imaging (9 spectral bands from each of the two light sources). The smoothness score [188], $S_{\text {smooth }}$, was defined as:

$$
S_{\text {smooth }}=1-\left(\frac{\sigma-\sigma_{\min }}{\sigma_{\max }-\sigma_{\min }}\right)
$$

where $\sigma_{\max }$ and $\sigma_{\min }$ are the maximum and minimum standard deviations calculated across all correction methods, such that scores of 1 and 0 represent the best and worst smoothness achieved respectively. 


\section{Signal}

It is crucial that decombing and demosaicking preserve regions of high pixel intensity, as detection of these high signal regions is central to techniques such as optical molecular imaging (OMI), autofluorescence imaging and reflectance imaging. Fluorescence emission was chosen as the source used to quantify signal here, as it is relevant to our work on OMI (Chapter 4), but ultimately, the physical source of the regions of high pixel intensity should not alter the results. Fluorescence signals were acquired by capturing images of a $30 \mu \mathrm{L}$ solution of $1 \mathrm{mg} / \mathrm{mL}$ of the fluorescent dye AF647 (Thermo Fisher Scientific, USA) dissolved in phosphate buffered saline (PBS) in a well plate ( $\mu$-Slide 18 Well Flat, ibidi GmbH, Germany) using illumination from a $635 \mathrm{~nm}$ LED (UHP-T-LED-635-EP, Prizmatix, Israel) and a long pass emission filter in front of the camera (ET700/75m, Chroma, USA). The mean pixel intensity, $S$, was calculated in a region of interest (ROI) drawn manually inside the well on the image. For multispectral imaging, the mean pixel intensity was extracted from those bands that overlap with the emission spectrum of AF647 (narrow bands: 665 nm, 714 nm, FWHM, 27 nm, 26 nm; broadband: 500$850 \mathrm{~nm})$. The signal score, $S_{\text {signal }}$, was defined as:

$$
S_{\text {signal }}=\frac{S-S_{\min }}{S_{\max }-S_{\min }}
$$

where $S_{\max }$ and $S_{\min }$ are the maximum and minimum signals calculated across all correction methods, such that scores of 1 and 0 represent the best and worst signal achieved respectively.

\section{Speed}

Speed was determined by measuring the total computation time to correct 10 images in series on a MacBook Pro (Processor 2.4 GHz Intel Core i5, Memory 8 GB 1600 MHz DDR3) and then dividing by 10 to calculate the time per frame.

\section{E. Accuracy of spectral reconstruction (multispectral performance metric only)}

For multispectral imaging, it is crucial that the information from different pixels on the SRDA is not mixed by the comb correction process. In order to assess the performance of each correction method, a score to represent the accuracy of spectral reconstruction (ASR) was defined. To extract this score, the following steps were performed:

i. Using the multispectral endoscope, an image of a white reflecting target (paper), illuminated by a narrowband source (UHP-T-LED-635-EP, Prizmatix, Israel) coupled to the PolyScope illumination fibre, was captured. 
ii. This image was demosaicked and decombed as outlined in Section 3.3.4 to provide a multispectral cube of data: $I^{k}, k=1-9$ where $k$ indicates the spectral band.

iii. The 'ground truth' spectral properties of the image data were determined. The spectrum, $G(\lambda)$, of the target was captured using a reference spectrometer (AvaSpec-ULS2048, Avantes, Netherlands).

iv. The 'ground truth' spectrum was multiplied by the response of the endoscope in each spectral band $k, R^{k}(\lambda)$, to predict the 'ground truth' recorded spectrum:

$$
E^{k}=\sum_{\lambda} G(\lambda) R^{k}(\lambda), \quad k=1-9
$$

v. The normalised (to AUC=1) average (over all pixels in the image) spectrum collected with the endoscope was compared to the predicted 'ground truth' spectrum and the mean squared difference was determined by:

$$
\left.Q=\sum_{k=1}^{9} \overline{\left(\frac{\sum_{x, y} I^{k}(x, y)}{\sum_{x, y} 1}\right)}-\overline{E^{k}}\right)^{2}
$$

where the bar represents normalisation of spectra to $\mathrm{AUC}=1$.

vi. The accuracy of spectral reconstruction score, $S_{A S R}$, is defined as:

$$
S_{A S R}=1-\left(\frac{Q-Q_{\min }}{Q_{\max }-Q_{\min }}\right)
$$

where $Q_{\max }$ and $Q_{\min }$ are the maximum and minimum mean squared differences calculated across all correction methods, such that scores of 1 and 0 represent the best and worst accuracy of spectral reconstruction achieved respectively.

By spatially (pixel-by-pixel) averaging the spectra prior to normalisation and calculation of the mean square difference, the influence of non-smoothness of the images is reduced, since this effect is already accounted for in the smoothness metric.

\subsubsection{Overall Performance}

Since there are trade-offs between the performances of the metrics, the overall performance of a particular correction method depends on which of the metrics are prioritised in a given application. To account for this, an overall performance score, $O P$, was constructed:

$$
O P=w_{\text {res }} \mathrm{S}_{\text {res }}+w_{\text {smooth }} S_{\text {smooth }}+w_{\text {signal }} S_{\text {signal }}+w_{A S R} S_{A S R}
$$


with adjustable application-dependent weightings, $w$, to emphasize a priority metric, such that:

$$
w_{\text {res }}+w_{\text {smooth }}+w_{\text {signal }}+w_{\mathrm{ASR}}=1
$$

Speed was not included in $O P$ since it is possible to independently optimize speed by improving hardware and parallelizing software. For monochrome imaging $w_{A S R}=0$. Since weightings are application-dependent, the $O P$ for all weightings was calculated, and displayed these using a colour map such that the reader may visually select the optimum correction method.

\subsection{Comb Correction Results}

\subsubsection{Performance of Simulated Monochrome Image Corrections}

The scores for resolution, smoothness and signal as a function of the characteristic filter size for 4 decombing methods tested in simulated monochrome images were calculated for three different sized comb structures, $M=0.7, M=1.0$ and $M=1.3$ (Figure 3.9). These structure sizes were chosen to represent a range from 7500-30000 fibrelets per bundle, magnified to fill a similar $\sim 1000 \times 1000$ pixel sensor. For physical blurring, the correction occurs in hardware at the point of imaging. The current approach does not replicate the full imaging process, so accurate simulation of physical blurring was not possible. Speed was not tested with simulated mages, since the size of the comb structure makes no difference to computation time.

These simulations revealed that the size of the fibrelet has little effect on the overall trends in performance of the different methods. This is confirmed in Figure 3.10, which shows the overall performance for the different fibrelet sizes. The general shape of the optimal performance space remains constant, with the only change being a preference for median filtering in a tiny region of the performance space (around $w_{\text {smooth }}=0.85, w_{\text {signal }}=0.15$ ) for the smaller fibrelet dimensions ( $\mathrm{M}=0.7)$. This is because median filtering performs better when the filling factor, the fraction of the image filled with data (fibrelet) versus artefact (cladding), is larger, as is the case for the smaller fibrelet size. This is also observed in Figure 3.9, where the $M=0.7$ median data follows a different trend to the $\mathrm{M}=1.0$ and $\mathrm{M}=1.3$ data, giving a higher signal score.

Since fibre diameter made little difference to the overall layout of performance space, we continued our investigation using experimental data from our endoscope, which produced images corresponding to $\mathrm{M}=1.0$. 


\subsubsection{Performance of Experimentally Captured Monochrome Image Corrections}

The scores for resolution, smoothness, signal and speed as a function of the characteristic filter size for the 5 decombing methods tested in monochrome imaging enable direct comparisons to be made regarding the individual performance of the methods (Figure 3.11). Example images are shown in Figure 3.12.

Similar trends are observed for performance of corrections in experimental image data (Figure 3.11) and in simulated image data (Figure 3.9, Figure 3.10). The main difference between simulation and experiment is that interpolation appears to achieve an enhanced resolution score in experimental data compared to simulated data, suggesting the noise component of simulated data may have been slightly overestimated. Overestimation of the noise component would disproportionately degrade the performance of interpolation relative to the other correction methods; it is more susceptible to noise as it relies on data from a single pixel per fibrelet.

The results from the monochrome data corrections are summarised in Figure 3.11. For preservation of resolution and signal, interpolation clearly provides the optimal solution. If image smoothness is our only priority, Gaussian filtering is preferred. Otherwise, Fourierfiltering provides a compromise between smoothness and resolution. 

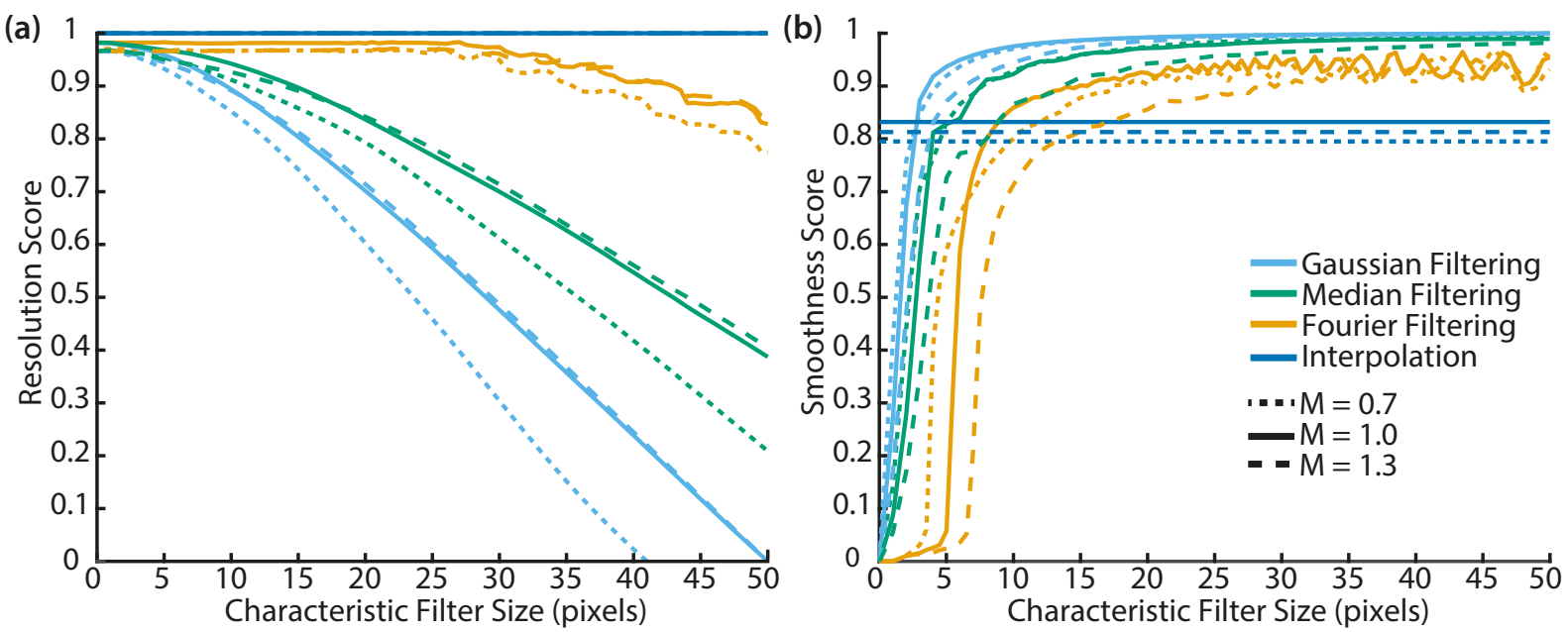

(c)

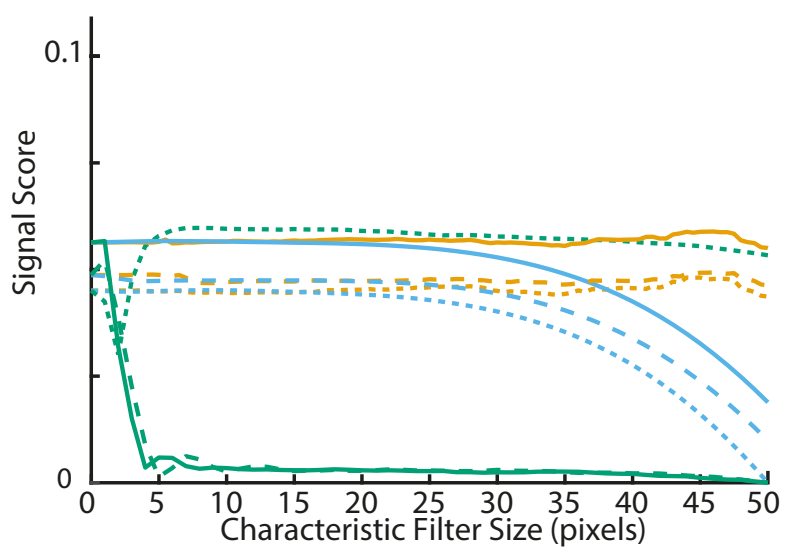

Figure 3.9 Performance scores for 4 correction methods applied to simulated monochrome images for $M=0.7, M=1.0$ and $M=1.3$.

(a) Resolution Score. $R_{\max }=82,102,103$ pixels $(648,803,809 \mu \mathrm{m})$ and $R_{\min }=21.4,22.4,23.5$ pixels $(169$, $177,185 \mu \mathrm{m}$ ) for $\mathrm{M}=0.7,1.0,1.3$ respectively. (b) Smoothness Score. $\sigma_{\max }=20.3,19.9,20.1$ and $\sigma_{\min }=0.638,0.388,0.346$ for $\mathrm{M}=0.7,1.0,1.3$ respectively. (c) Signal Score. $\mathrm{S}_{\max }=151,156,156$ and $\mathrm{S}_{\min }=118,117,118$ for $\mathrm{M}=0.7,1.0,1.3$ respectively. Dotted line $\mathrm{M}=0.7$, solid line $\mathrm{M}=1.0$, dashed line $\mathrm{M}=1.3$ Since interpolation between irregularly spaced points is a complex spatially variant filter, the characteristic filter size is not well defined, so the score for interpolation is represented as horizontal line in each graph. 
(a) $M=0.7$

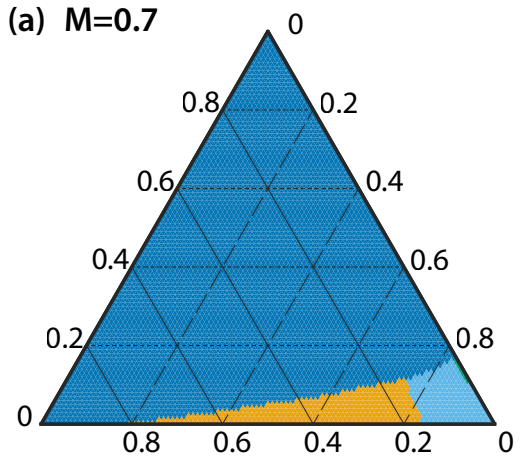

(b)

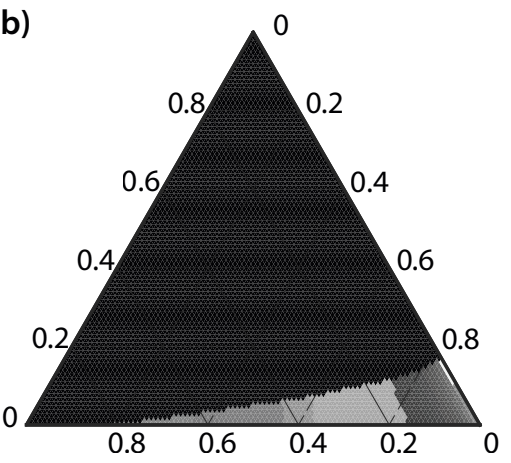

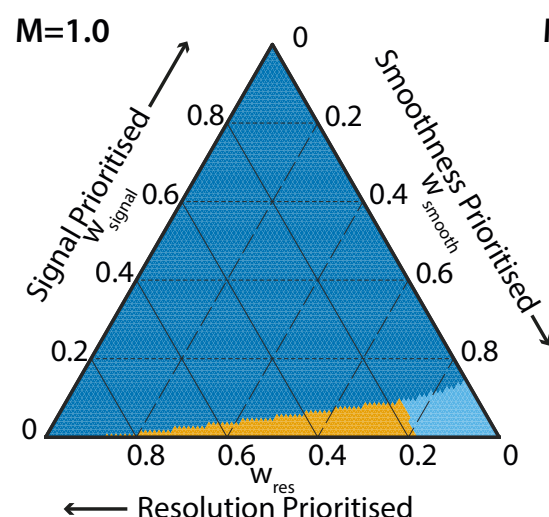
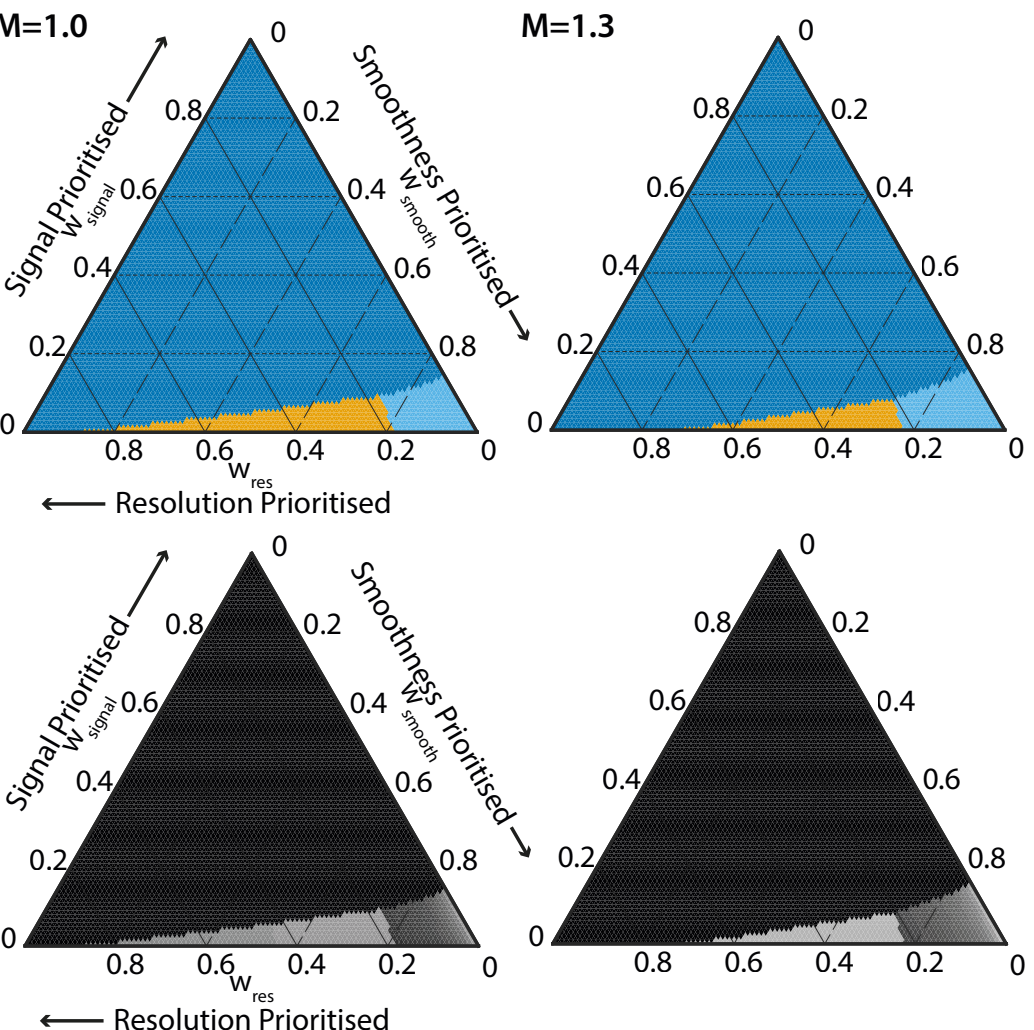

lines of constant $w_{\text {res }}$ ines of constant $w$ lines of constant $w_{\text {signal }}$

Characteristic Filter Size

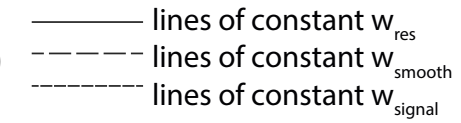

Gaussian Filtering Median Filtering

Fourier Filtering Interpolation

Figure 3.10 Optimum correction method based on overall performance score for simulated images with $M=0.7, M=1.0$ and $M=1.3$.

(a) The correction method that gives highest overall performance (OP). (b) The characteristic filter size used with this correction method to achieve the highest OP. Since interpolation between irregularly spaced points is a complex spatially variant filter, the characteristic filter size is not well defined, so it is represented as zero in the graphs. Speed is not included in the OP since it is possible to optimise speed independently. 
(a)

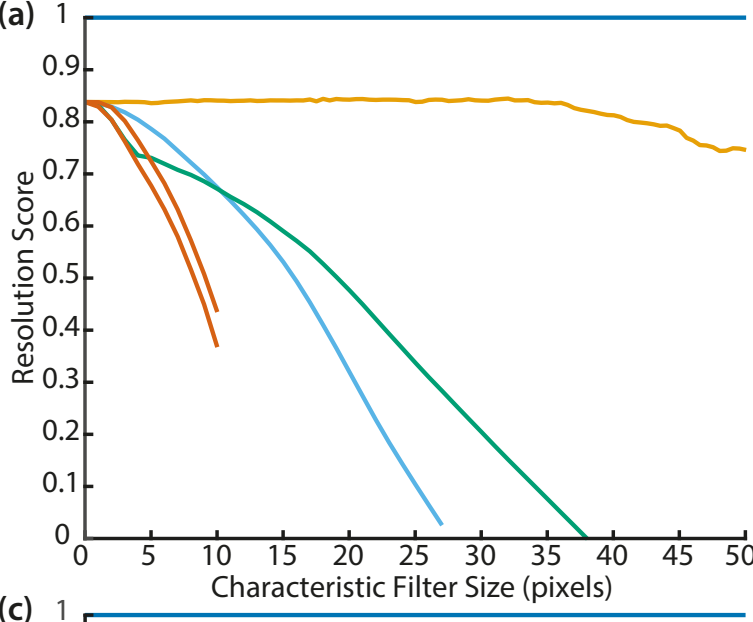

(c)

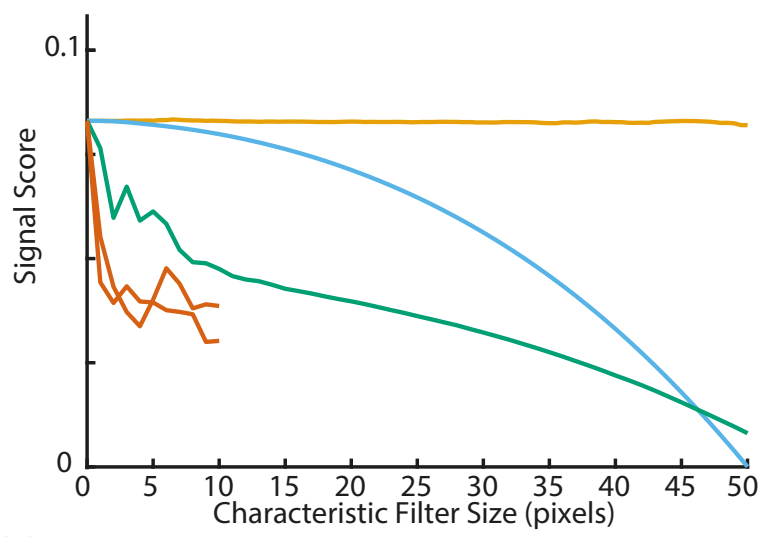

(b) 1

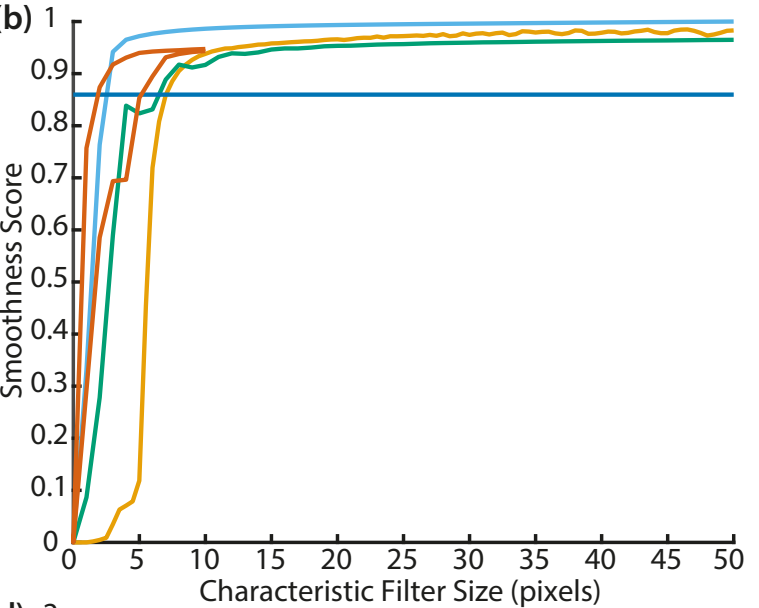

(d) 2

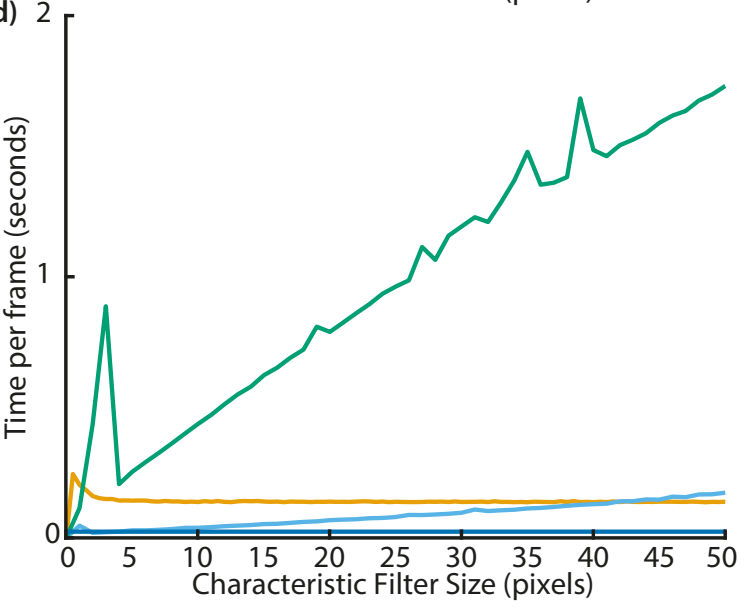

(e)

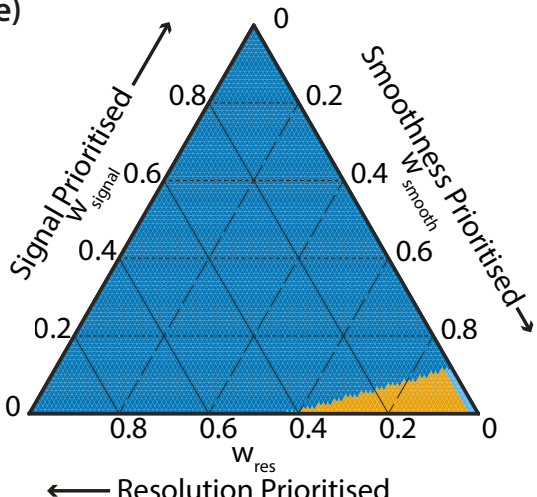

(f)

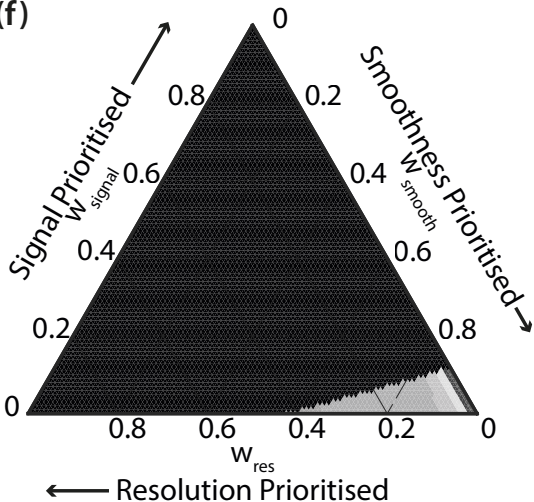

Gaussian Filtering Median Filtering Fourier Filtering Interpolation Physical Blurring

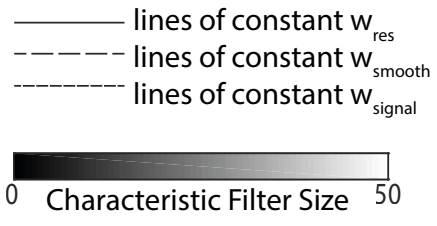

Figure 3.11 Performance scores for 5 correction methods applied to monochrome images.

(a). Resolution Score. $R_{\max }=500 \mu \mathrm{m}$ and $\mathrm{R}_{\min }=228 \mu \mathrm{m}$. (b) Smoothness Score. $\sigma_{\max }=32.2$ and $\sigma_{\min }=3.85$. (c) Signal Score. $S_{\max }=157$ and $S_{\min }=114$. (d) Time to correct each frame. Since interpolation between irregularly spaced points is a complex spatially variant filter, the characteristic filter size is not well defined, so the score for interpolation is represented as horizontal line in each graph. (e) Optimum correction method based on highest overall performance score OP for weightings $\mathrm{W}_{\text {res, }} \mathrm{W}_{\text {smooth }}$ and $\mathrm{W}_{\text {signal }}$. (f) The characteristic filter size used with this correction method to achieve the highest overall performance (OP). Since interpolation between irregularly spaced points is a complex spatially variant filter, the characteristic filter size is not well defined, so the score for interpolation is represented as zero the graphs. Speed is not included in the OP since it is possible to optimize speed independently. 


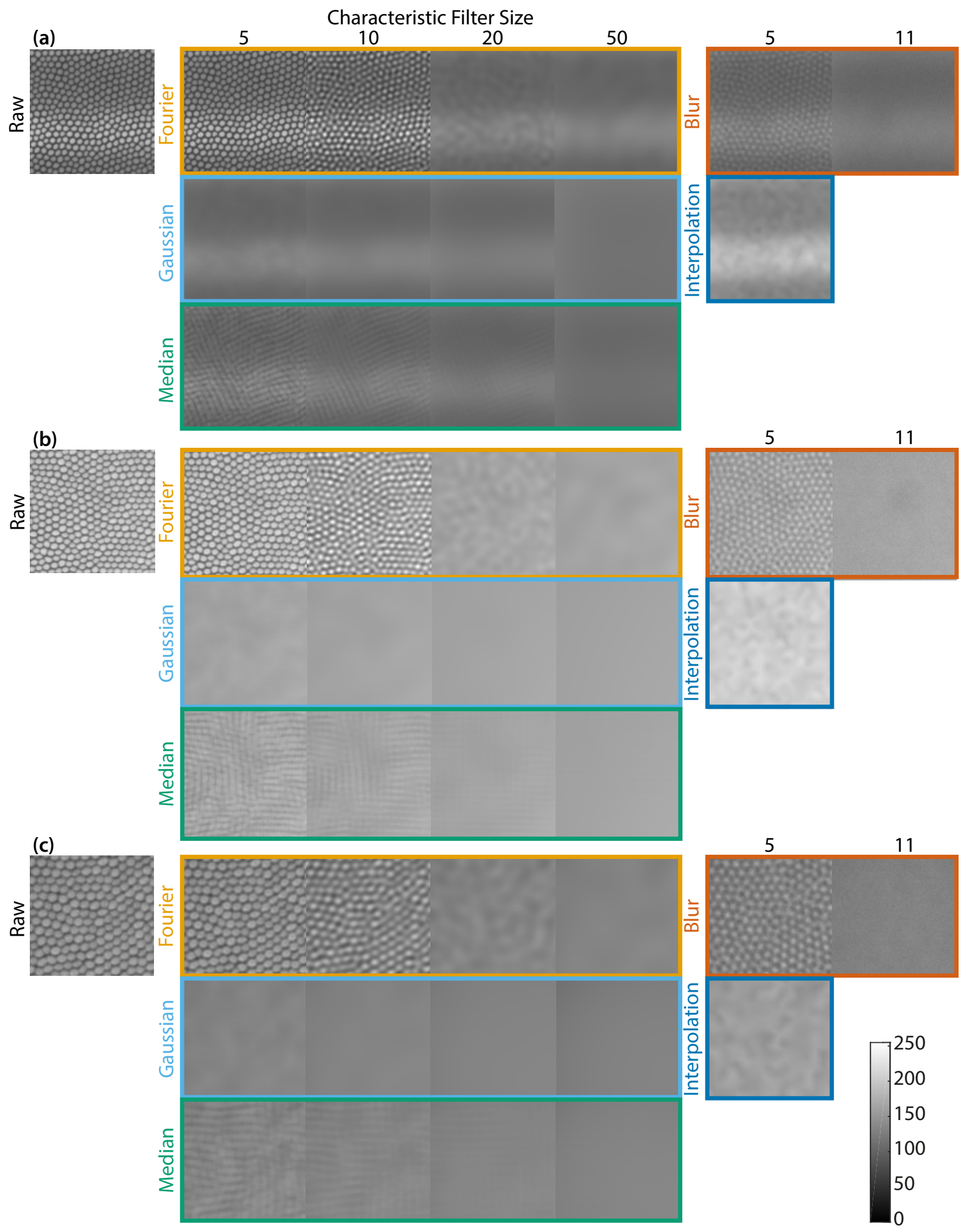

Figure 3.12 Example corrected monochrome images.

(a). Region of an image of a USAF test chart element used to determine resolution. (b). Image of white reflecting target (paper) used to determine smoothness. (c). Image of AF647 used to determine signal. 


\subsubsection{Performance of Captured Multispectral Image Corrections}

The scores for resolution, smoothness, signal, ASR and speed as a function of the characteristic filter size for the 5 decombing and demosaicking methods tested in multispectral imaging are shown in Figure 3.13. The ASR score is highest for interpolation, which is expected since interpolation removes the erroneous pixels from the cladding region. The other methods yield reduced ASR scores since they mix together the 'true' spectra from fibrelet centres and the 'erroneous' spectra from the cladding, which is particularly evident in median filtering, where the sparsity of 'true' pixels, from the compound sampling of mosaic and comb can result in median filters removing 'true' pixels over the more common 'erroneous' pixels, and in physical blurring, where the 'true' and 'erroneous' spectra are mixed before detection.

The computation time in multispectral corrections is increased by around one order of magnitude, as expected since there are nine images to correct rather than one. The computation time of physical blurring represents demosaicking alone. If direct demosaicking were performed, resulting in 9 images each with a 9-fold reduction in total image pixels, the computation time would be significantly reduced.

The overall performance is shown in Figure 3.14. In the context of multispectral imaging, it was found that interpolation frequently provides the optimal solution for correction as was the case for monochrome imaging. Only when smoothness is prioritised does interpolation fail to provide the best solution. In this special case, Gaussian, median or Fourier filtering would be preferred, with Fourier filtering providing a better solution preservation of resolution is also desired. Example images are shown in Figure 3.15. 

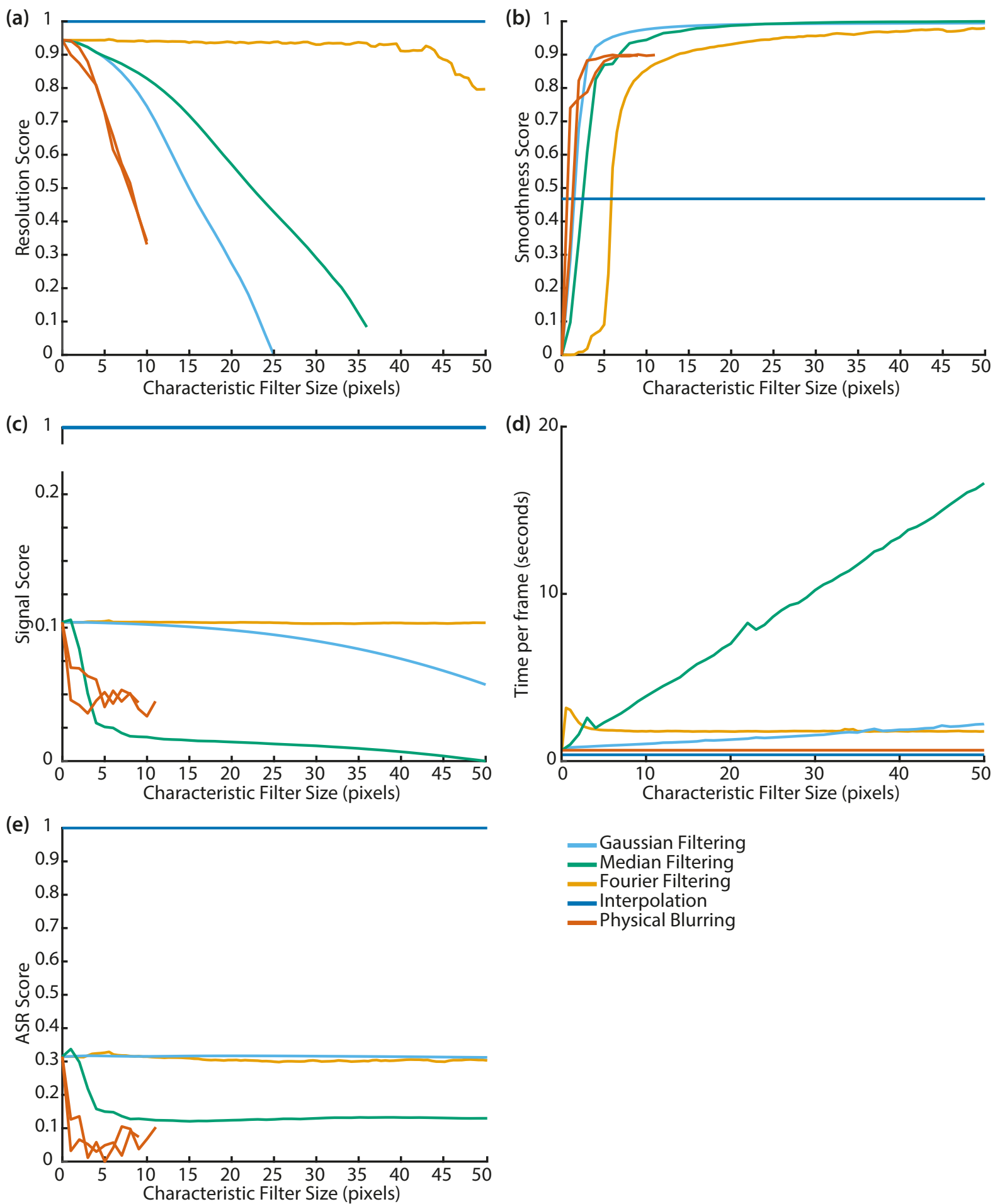

\section{Figure 3.13 Performance scores for 5 correction methods applied to multispectral images for multispectral images.}

(a) Resolution score. $R_{\max }=440 \mu \mathrm{m}$ and $R_{\min }=260 \mu \mathrm{m}$. (b) Smoothness score. $\sigma_{\max }=13$ and $\sigma_{\min }=0.73$. (c) Signal score. $\mathrm{S}_{\max }=28.1$ and $\mathrm{S}_{\min }=10.6$. (d) Speed. (e) Accuracy of spectral reconstruction. $\mathrm{Q}_{\max }=0.0138$ and $\mathrm{Q}_{\min }=0.0060$. Since interpolation between irregularly spaced points is a complex spatially variant filter, the characteristic filter size is not well defined, so the score for interpolation is represented as horizontal line in each graph. 

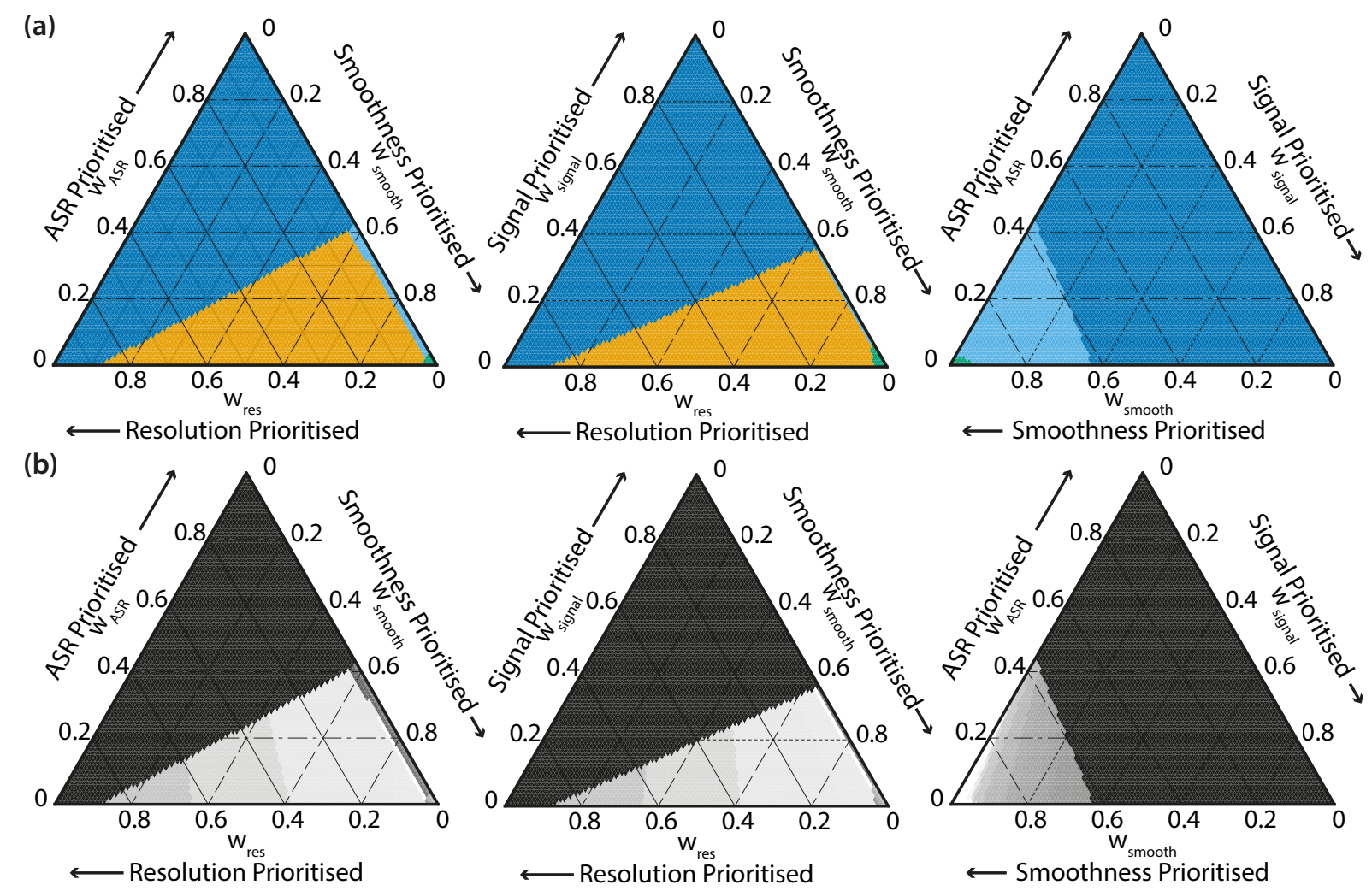

$\longleftarrow$ Smoothness Prioritised

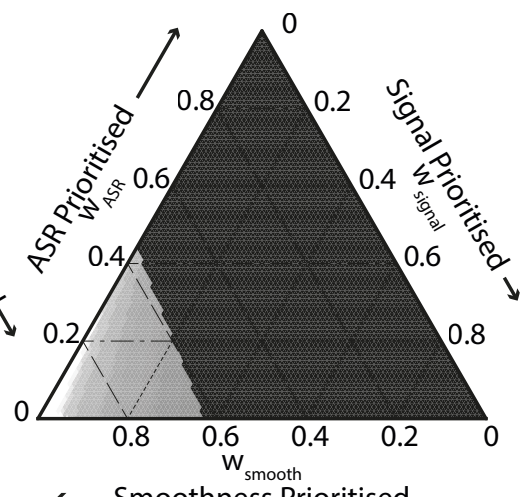

Gaussian Filtering Median Filtering

Fourier Filtering

Interpolation
Physical Blurring

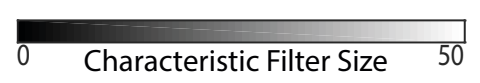

$\longleftarrow$ Smoothness Prioritised

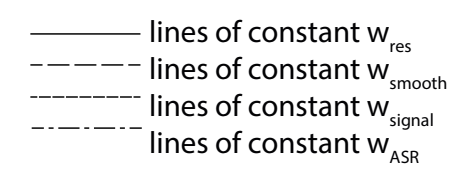

Figure 3.14 Optimum correction method based on overall performance score.

(a) The correction method that gives highest overall performance (OP). (b) The characteristic filter size used with this correction method to achieve the highest OP. For each ternary plot, the weighting not shown on the axes is set to zero. Since interpolation between irregularly spaced points is a complex spatially variant filter, the characteristic filter size is not well defined, so the score for interpolation is represented as zero in the graphs. Speed is not included in the OP since it is possible to optimise speed independently. The trade-off between resolution, signal and ASR, is not shown since in all combinations interpolation gives the best overall performance. 
(a) Reference Image

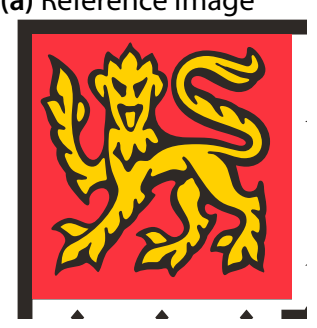

(b) Raw Captured Image

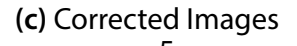
5

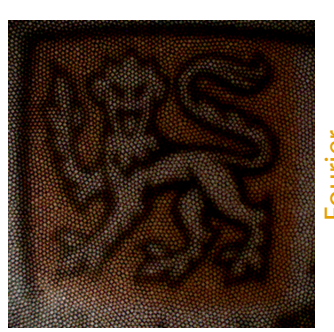

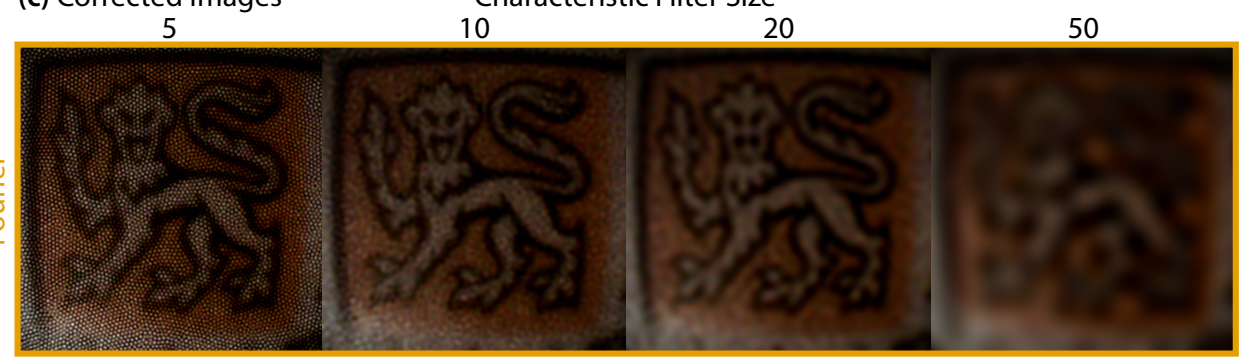

Characteristic Filter Size 10
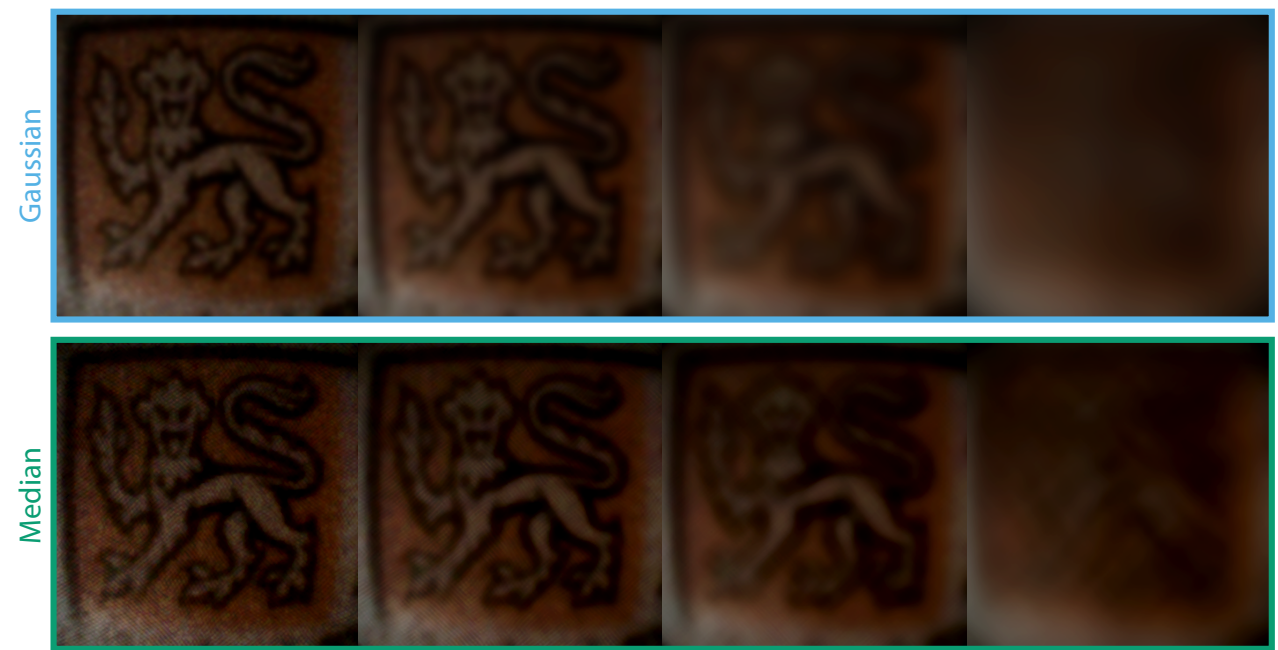

4 10
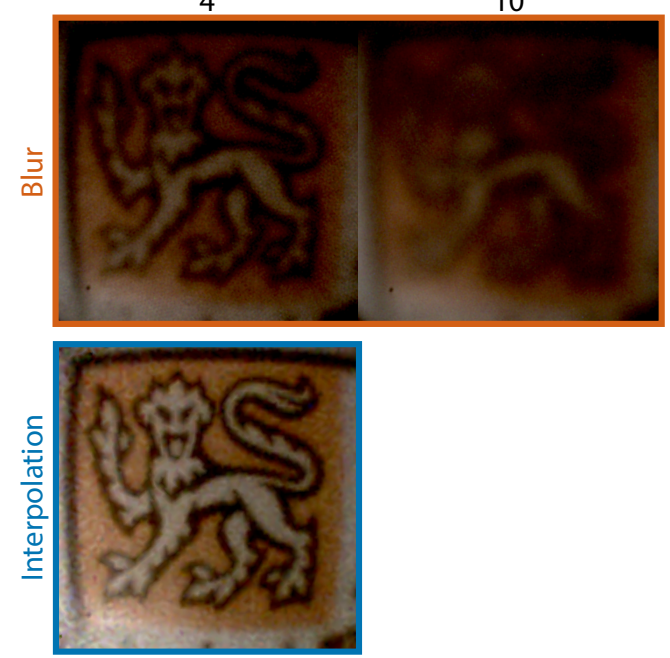

Figure 3.15 Example corrected multispectral images.

(a) A section of the University of Cambridge crest. This was printed and imaged with the multispectral endoscope. (b) The image generated by demosaicing the raw captured image and assigning the narrow bands centred at 629, 587 and $553 \mathrm{~nm}$ to RGB channels respectively. (c) Corrected images. 


\subsection{Discussion and Conclusions}

The clinically approved PolyScope disposable endoscope allows light to be delivered to, and collect light from, the oesophagus, enabling the development and in vivo evaluation of novel optical imaging techniques with the capacity for swift translation. However, the use of a fibre bundle introduces a comb structure into the images, which must be removed by image decombing algorithms to improve image quality to a level that is satisfactory for the end user. This process is further complicated in SRDA-based multispectral imaging, where it must be performed alongside demosaicking. In order to address this problem, the performance of commonly used methods of decombing was evaluated and compared in monochrome imaging and SRDA-based multispectral imaging. By defining scores relating to resolution, smoothness, signal, speed and accuracy of spectral reconstruction, each of these correction methods was evaluated within the parameter space of relevance for fiberscope imaging. Through data acquired with simulation and imaging, it was shown that interpolation provides the optimal solution in most cases for both monochrome and multispectral imaging. Only when image smoothness is highly prioritised, is Gaussian filtering preferred, while Fourier-filtering can be used in cases requiring a compromise between smoothness and resolution.

The optimal choice of correction method is application-dependent, hence rather than providing absolute recommendations, overall performance scores composed of weighted sums of performance metrics were objectively prepared. Weightings can therefore be assigned depending on the imaging priorities in a given application, which will vary significantly. To give some examples, visual inspection in real time puts speed as a high priority while inspection of detailed surface features, such as mucosal patterns or vasculature, will put resolution as a high priority. For autofluorescence imaging or fluorescence molecular imaging, where signal is relatively limited due to low abundance of fluorescence molecules in vivo, signal should be maximised to facilitate detection of these fluorescence markers. In cases requiring supervised classification of spectral signatures, such as in evaluation of oxy- and deoxy-haemoglobin concentrations during multispectral imaging, the accuracy of the spectral reconstruction is vital.

While the results presented here enable comparison of the performance of these different correction methods across a range of applications, there remain some limitations to this study. Firstly, only the case where the fibrelet image size on the sensor is greater than the size of a super-pixel $(T<1)$ was considered. In practical applications, appropriate lenses could be used to magnify the image to ensure that this constraint is fulfilled. A consequence of this constraint is that demosaicking should not affect resolution. Indeed, the monochrome sensor and multispectral sensors had maximum resolutions of $228 \pm 18 \mu \mathrm{m}$ and $240 \pm 20 \mu \mathrm{m}$ respectively 
(errors determined from errors in a linear fit to Michelson contrast data). Secondly, the magnification approach that was used in simulation does not quite recapitulate all possible combs, since it magnifies both the cores and the cladding, whereas in reality, the same amount of cladding may be used or it may scale differently to the cores. Finally, the effect of compound methods such as interpolation followed by Gaussian smoothing was not considered.

In the chapters that follow, work developing two optical endoscopic imaging techniques based on the PolyScope is described. Both of these techniques aim to detect signal from fluorescence or reflectance, meaning resolution is considered secondary to signal and accuracy of spectral reconstruction, so unless otherwise stated, interpolation based decombing and demosaicing is applied. By using the PolyScope to deliver to and collect light from the oesophagus, it was possible to quickly translate these techniques to first-in-human trials. 



\section{FLEXIBLE ENDOSCOPY: OPTICAL MOLECULAR IMAGING}

This chapter uses material from two papers: an original research paper by the author entitled "Design and validation of a near-infrared fluorescence endoscope for detection of early esophageal malignancy"; and a co-authored paper entitled "Detection of early neoplasia in Barrett's esophagus using lectin-based near-infrared imaging: an ex vivo study on human tissue" (see publications list).

\subsection{Molecular Imaging for Endoscopic Surveillance of Barrett's Oesophagus}

Targeted molecular imaging agents [21] require synthesis at good manufacturing practice (GMP) standards, associated toxicology studies, specific instrumentation, and add time and cost to procedures (Translational Characteristic 1) [20,65]. Despite these challenges, molecular imaging is an attractive technique as it enables macroscopic visualisation of complex biochemical processes [21]. That is, rather than probing some macroscopic proxy of underlying biochemical change as does HD-WLE or NBI, molecular imaging allows a specific intrinsic molecular change associated with disease to be probed, meaning it has the potential to allow highly specific red flag surveillance of the oesophagus. Efforts in the development of molecular imaging contrast agents for early detection of dysplasia in Barrett's oesophagus are summarised in Table 4.1. 


\section{Table 4.1 Optical molecular imaging techniques in trials for surveillance of Barrett's oesophagus.}

$\uparrow:$ up-regulated/over-expressed, $\downarrow$ : down-regulated/under-expressed, $\gamma$-Glut: $\gamma$-Glutamyltranspeptidase, FITC: fluorescein isothiocyanate, EGFR: epidermal growth factor receptor, HER2: human epidermal growth factor receptor 2, Cyp-A: cyclophilin A, EMRs: endoscopic mucosal resections, IV: intravenous.

\begin{tabular}{|c|c|c|c|c|c|c|c|}
\hline Target & Probe & $\begin{array}{l}\text { Optical } \\
\text { Reporter }\end{array}$ & Device & Subjects & Application & FOV & Prospect \\
\hline $\begin{array}{l}\text { Cyp-A and cell surface } \\
\text { protein CD147 ( } \uparrow) \text { [199] }\end{array}$ & $\begin{array}{l}\text { Peptide: } \\
\text { ASYNYDA }\end{array}$ & FITC & $\begin{array}{l}\text { GastroFlex type MiniOminiprobe } \\
\text { (Mauna Kea Technologies), } \\
\text { Custom Device [145] }\end{array}$ & in vivo & Topical & $\begin{array}{l}\text { Narrow/ } \\
\text { Wide }\end{array}$ & ++ \\
\hline Surface glycans $(\downarrow)$ [200] & $\begin{array}{l}\text { Lectin: Wheat Germ } \\
\text { Agglutinin (WGA) }\end{array}$ & $\begin{array}{l}\text { AlexaFluor } \\
488\end{array}$ & $\begin{array}{l}\text { Gastrointestinal videoscope } \\
\text { (GID FQ260Z, Olympus), } \\
\text { Custom Device [201] }\end{array}$ & $\begin{array}{l}\text { Whole } \\
\text { Organ } \\
\text { ex vivo }\end{array}$ & Topical & Wide & ++ \\
\hline $\begin{array}{l}\text { Cathepsin B (CTSB) }(\uparrow) \\
{[202]}\end{array}$ & Activatable Probe: Prc & osense 750 & Custom Device [203] & Mice & IV & Wide & + \\
\hline HER2 ( $\uparrow)[204]$ & $\begin{array}{l}\text { Antibody: } \\
\text { anti-HER2 }\end{array}$ & $\begin{array}{l}\text { AlexaFluor } \\
488\end{array}$ & $\begin{array}{l}\text { Cellvizio Confocal } \\
\text { CholangioFlex } \\
\text { (Mauna Kea Technologies) }\end{array}$ & Rats & IV & Narrow & - \\
\hline EGFR, Survivin ( $\uparrow$ ) [205] & $\begin{array}{l}\text { Antibodies: anti- } \\
\text { human-EGF-R, anti- } \\
\text { human-survivin }\end{array}$ & Fluorescein & $\begin{array}{l}\text { Cellvizio GastroFlex UHD } \\
\text { (Mauna Kea Technologies) }\end{array}$ & Pigs & Topical/IV & Narrow & - \\
\hline Unknown [206] & $\begin{array}{l}\text { Peptide: } \\
\text { SNFYMPL }\end{array}$ & FITC & $\begin{array}{l}\text { Axioskop } 2 \text { plus microscope } \\
\text { (Zeiss) }\end{array}$ & $\begin{array}{l}\text { EMRs } \\
\text { ex vivo }\end{array}$ & Topical & Narrow & - \\
\hline
\end{tabular}

Application of intravenously administered probes is limited as they require at least 24 hours between injection and endoscopy, hindering clinical workflow [202, 204]. Encouragingly, labels that can be topically applied during the endoscopic procedure have begun to emerge. A fluorescently labelled peptide that binds specifically to high-grade dysplasia and adenocarcinoma has recently been shown by Sturm et al. to delineate these pathologies in vivo in patients [199], however, the sensitivity to low-grade dysplasia has yet to be established. A follow-up study by Joshi et al., using a wide-field fluorescence imaging endoscope, noted some important limitations, including the challenge in visualising the target fluorescence due to strong tissue autofluorescence, and the resulting low values of target-to-background ratio from the diagnostic fluorescence features [145] . Molecular imaging probes have also been combined with endomicroscopy in several studies [205, 206], but these techniques have a narrow field of view, limiting them to performing optical biopsy rather than red flag imaging.

\subsection{The Potential of Fluorescently Labelled Lectins}

Bird-Lieberman et al. demonstrated that cell-surface glycans are altered in the progression from Barrett's oesophagus to adenocarcinoma (Figure 4.1 (a)). The lectin wheat germ agglutinin (WGA) showed specific changes in binding patterns between high-grade and low-grade dysplastic tissue and non-dysplastic Barrett's oesophagus [200]. WGA has great potential for clinical translation as an OMI contrast agent (Translational Characteristic 1): it can be applied topically using a spray catheter; it requires only 10 minutes incubation time, minimising the 
disruption to the normal clinical workflow; it is easily displaced by washing with an excess of glucosamine following imaging; it is inexpensive, stable at high temperature, stable at low $\mathrm{pH}$ and resistant to proteolysis; due to its large size (37,300 Da), its binding is less susceptible to alteration once it is conjugated to a fluorescent dye; and lectins are part of a normal human diet and thus have low toxicities if used at sufficiently low concentrations [200].

Next, Bird-Lieberman et al. conjugated WGA to AlexaFluor 488, a commercially available fluorescent dye with excitation peak around $488 \mathrm{~nm}$. This was topically applied to resected oesophagi and imaged in a way that mimics an in vivo clinical study using a commercially available fluorescence endoscope (excitation 395-475 nm, detection 500-630 nm). Finally, the fluorescence signal was correlated to pathology. The results were promising but the signal to background ratios were low in some cases due to a high level of autofluorescence swamping the fluorescence signal from the lectin (Figure 4.1 (b),(c)) [200].

(a) Many targets present on Barrett's tissue Fewer targets present on dysplastic tissue so we get high fluorescence signal-
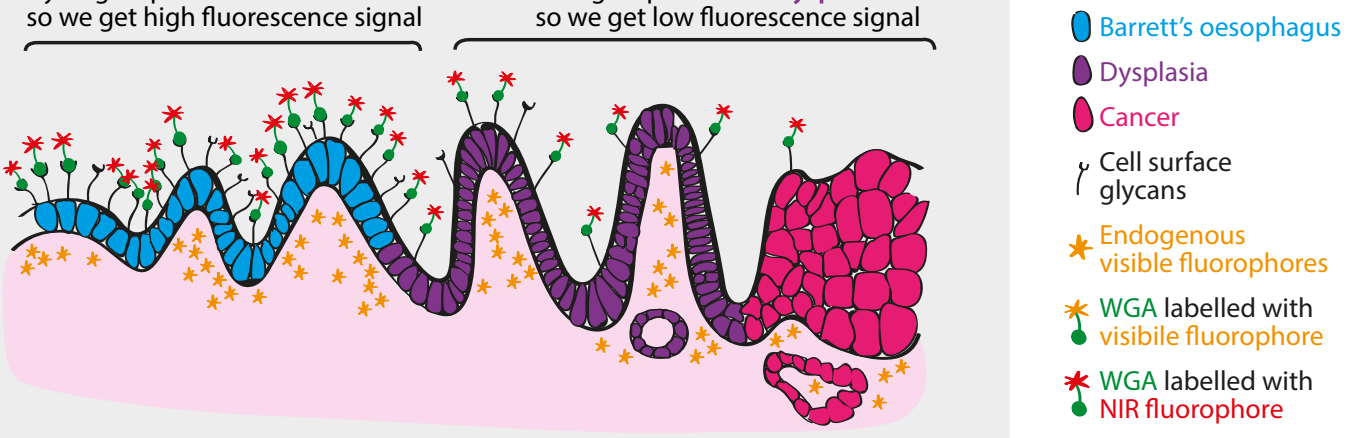

(b)

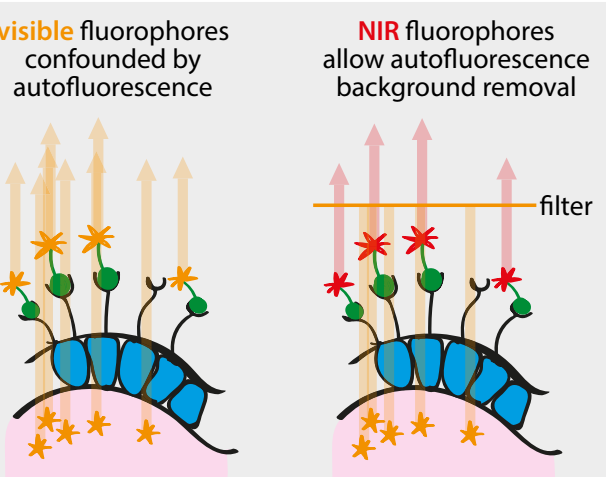

(c) Barrett's autofluorescence (excited at 400nm)
AlexaFluor 488 emission
IRDye 800CW emission

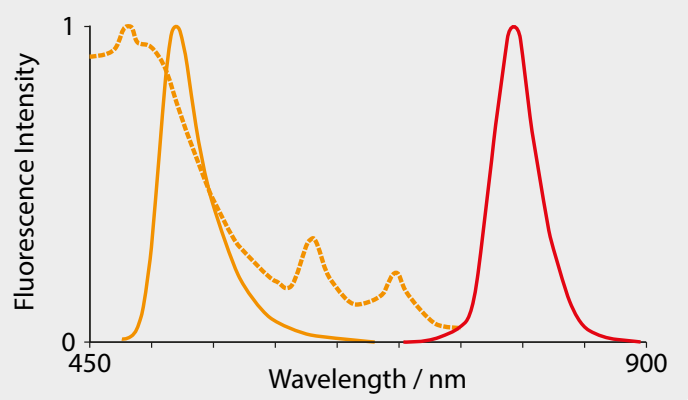

Figure 4.1 Optical molecular imaging with wheat germ agglutinin.

(a) Wheat germ agglutinin (WGA) shows specific changes in binding patterns between high-grade and low-grade dysplastic tissue and non-dysplastic Barrett's oesophagus [200]. (b) Imaging of WGA labelled with visible fluorophores, such as AlexaFluor 488, is confounded by autofluorescence. (c) Spectra show the overlap between autofluorescence and AlexaFluor 488 emission. IRDye 800CW has its emission peak in the near infrared, far from the autofluorescence of the Barrett's tissue. Autofluorescence data reproduced from [119]. The two small peaks around 630 and $700 \mathrm{~nm}$ are due to low dose injection of photofrin. AlexaFluor 488 and IRDye 800CW data from Chroma and LI-COR respectively [207, 208]. 
Progression of several promising molecular imaging techniques have encountered this same limitation. Existing endoscopy devices for detecting fluorescence emissions are optimised for detection of tissue autofluorescence in the visible wavelength range [26], meaning that the sensitivity, contrast and dynamic range for detection of exogenously applied contrast agents is limited by the high endogenous background signal present within the images. To avoid the autofluorescence background, imaging can be performed using near-infrared (NIR) fluorophores in the range $\sim 600-900 \mathrm{~nm}$, where the tissue is relatively devoid of endogenous fluorescence (Figure 4.1 (c)) [119]. Therefore, WGA was conjugated to a commercially available NIR fluorophore, IRDye 800CW-NHS ester dye (LI-COR, USA), to form WGAIR800 [209].

\subsection{Challenges in Realising the Potential of WGA-IR800}

Following the identification of a promising OIB, referring back to the OIB Roadmap (Figure 1.3), it needs to be established if a device exists to measure OIB. While several groups have developed flexible endoscopic devices for NIR fluorescence imaging in the gastrointestinal tract [73, 202, 210-212], there are currently no commercially available NIR fluorescence endoscopes for wide field surveillance of the oesophagus, so a collaboration to develop a NIR fluorescence endoscope in parallel to WGA-IR800 was initiated.

To enable clinical studies, both the device, and WGA-IR800 must be approved for in vivo trials. With regards to WGA-IR800, it was hoped that the combination of WGA's low toxicology profile and the GMP qualification of IR800 would facilitate this stage of translation. With regards to the device, to simplify approval, the NIR fluorescence endoscope was designed around the PolyScope accessory channel endoscope (Section 3.2). Since the PolyScope is CE marked, the NIR fluorescence endoscope requires only local approval by clinical engineering (Translational Characteristic 2).

To meet the experimental aims, the specification for the NIR fluorescence endoscope was as follows: it must be compact and robust enough to be deployed in a clinical environment; it must provide image guidance to the endoscopist so they are able to orient themselves in the oesophageal lumen; and it must be capable of acquiring wide field images of low concentrations of WGA-IR800, which is particularly challenging since most detectors are limited by reduced sensitivity in the NIR.

To meet these specifications, a compact bimodal endoscope was developed. The device is capable of detecting both NIR fluorescence for OMI, using a highly sensitive electron multiplying CCD (EMCCD), as well as traditional white light reflectance to enable guidance, 
using a second monochrome camera. The system was kept compact through the use of mirrors to bend the light path. With this device, NIR fluorescence imaging of WGA-IR800 has the potential to provide a wide field red flag surveillance technique capable of achieving high sensitivity and specificity for detection of dysplasia, improving the standard of care for Barrett's surveillance. The following sections detail the design, development and validation of this device in Domain 2 of the OIB Roadmap (Figure 1.3) in preparation for first-in-human trials.

\subsection{Materials and Methods}

\subsubsection{Fluorescent Lectin Synthesis}

André Neves (Senior Research Associate, CRUK Cambridge Institute) conjugated IRDye800CW NHS Ester (LI-COR, USA) with WGA (L9640, Sigma-Aldrich, USA) to produce WGA-IR800 according to the methods of Sato et al. [213].

\subsubsection{Endoscope Design}

The system is based around the PolyScope accessory channel endoscope described in Section 3.2 and is shown in Figure 4.2. Illumination is provided by a broadband halogen light source (OSL2B2, Thorlabs, Germany) clipped with a $750 \mathrm{~nm}$ short pass excitation filter (FESH0750, Thorlabs, Germany). Light is directed onto the sample through the light guide embedded inside the PolyScope catheter. Light collected by the imaging fibre is relayed through an infinity corrected 20× objective lens (421350-9970-000, Zeiss, Germany) and split into two channels with a long pass dichroic filter (DMLP650L, Thorlabs, Germany). Light with a wavelength in the range $400-633 \mathrm{~nm}$ is relayed by an achromatic doublet $(f=100 \mathrm{~mm}$, ACA254-100-A, Thorlabs, Germany) onto a grayscale CMOS sensor (Grasshopper3 GS3-U3-41C6M-C, PointGrey, USA) whilst light with a wavelength $>685 \mathrm{~nm}$ is relayed by an achromatic doublet ( $f=100 \mathrm{~mm}$, ACA254-100-B, Thorlabs, Germany) onto an electron multiplying CCD (EMCCD; ProEM+ eXcelon $512 \times 512$, Princeton, USA) through an $800 \mathrm{~nm}$ long pass emission filter (FELH0800, Thorlabs, Germany) allowing simultaneous white light reflectance and NIR fluorescence imaging.

The system is kept compact through the use of broadband mirrors (BB1-E02 and BB1-E03, Thorlabs, Germany) to bend the light path. The optics are securely housed inside a light tight enclosure and mounted on an optical breadboard (MB4545/M, Thorlabs, Germany) which is fixed to a stainless steel trolley (FW2901-3, Freeway Medical, UK) with a footprint of $512 \mathrm{~mm}$ $\times 480 \mathrm{~mm}$. This allows the system to be easily and safely transported around a crowded endoscopy suite (Translational Characteristic 2). 

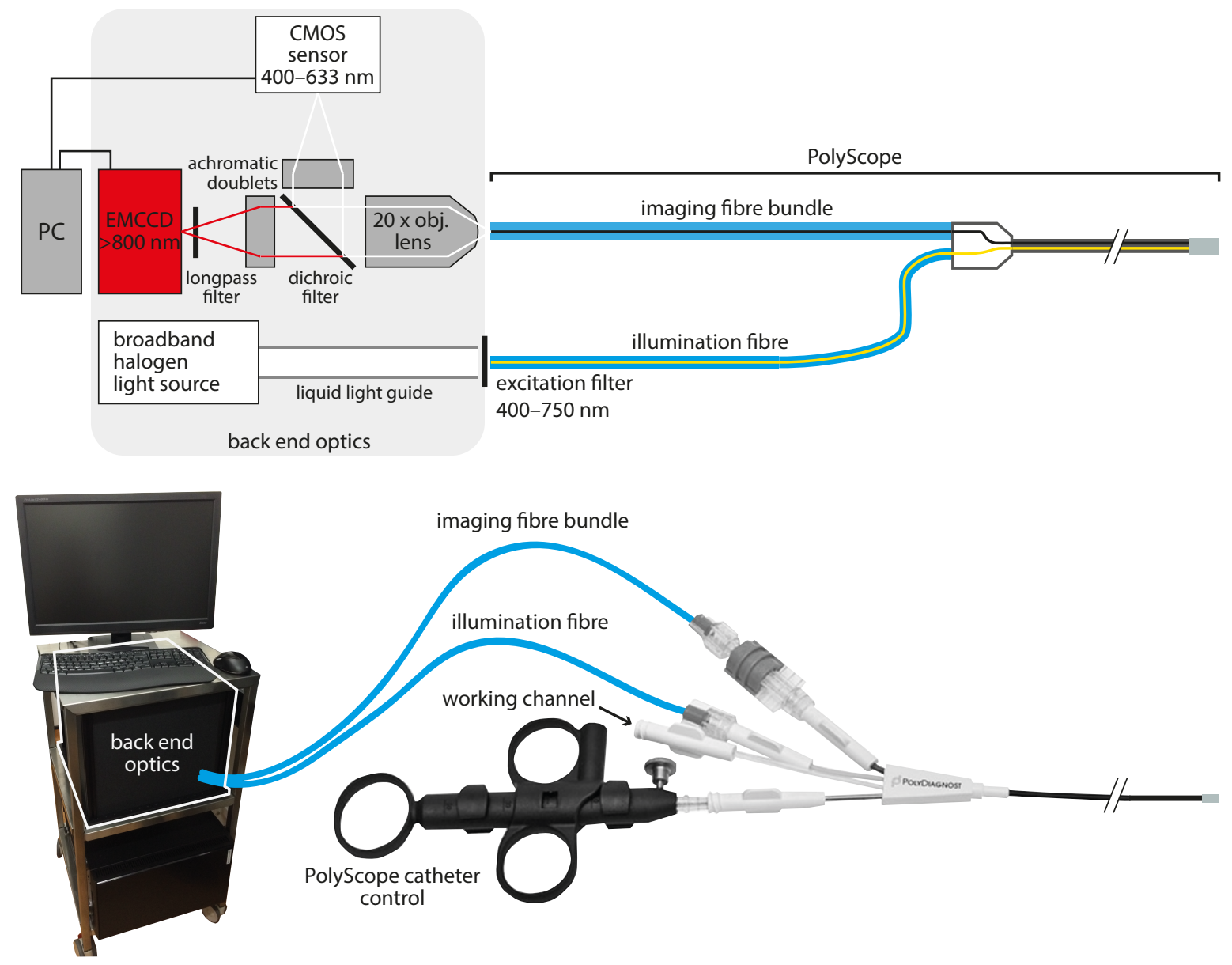

Figure 4.2 Schematic of the PolyScope based bimodal NIR fluorescence and WL reflectance endoscope.

The system is based around the PolyScope accessory channel endoscope (PolyDiagnost, Germany). Illumination is provided by a broadband halogen light source (OSL2B2, Thorlabs, Germany). Light from the PolyScope 10,000 fibre imaging bundle is focused by an infinity corrected $20 \times$ objective lens (4213509970-000, Zeiss, Germany) and split into two channels with a long pass dichroic filter (DMLP650L, Thorlabs, Germany). Light with a wavelength in the range $400-633 \mathrm{~nm}$ is relayed by an achromatic doublet lens ( $f=100 \mathrm{~mm}$, ACA254-100-A, Thorlabs, Germany) onto a grayscale CMOS sensor (Grasshopper3 GS3-U3-41C6M-C, PointGrey, USA) whilst light with a wavelength $>685 \mathrm{~nm}$ is relayed by an achromatic doublet ( $f=100 \mathrm{~mm}$, ACA254-100-B, Thorlabs, Germany) onto an electron multiplying CCD (EMCCD; ProEM+ eXcelon $512 \times 512$, Princeton, USA) through an $800 \mathrm{~nm}$ long pass emission filter (FELH0800, Thorlabs, Germany) allowing simultaneous white light reflectance and NIR fluorescence imaging. 


\subsubsection{Image Acquisition and Image Corrections}

A LabVIEW (National Instruments, USA) Visual Interface (VI) was created to control the cameras and acquire images. The white light images are saved as 8-bit $2048 \times 2048$ TIFF files and the NIR images were saved as 16-bit $512 \times 512$ TIFF files. Data analysis was carried out using Matlab® (MathWorks, USA). The comb structure introduced by the fibre bundle was removed using the interpolation methods outlined in Section 3.3.3. The white light and NIR images can be coregistered using the fibre locations, by selecting the four fibres located at the extreme $\mathrm{x}$ and $\mathrm{y}$ positions. The images are cropped at these locations and a similarity transformation is generated to successfully transform the white light image in $\mathrm{x}$ and $\mathrm{y}$ such that the extreme fibres are coregistered with the equivalent extreme fibres in the NIR image.

\subsection{Technical Characterisation}

Prior to translating a technique to the clinic, it is important to ensure it is well characterised, both with respect to its safety, for example by quantifying its illumination power (Section 4.5.1B), and with respect to its intended operation, for example by quantifying its field of view (Section 4.5.1A), resolution (Section 4.5.1C) and sensitivity (Section 4.5.1D). Whilst most of these are properties of the device, note that sensitivity is a combined characteristic of the device and molecular imaging probe.

\subsubsection{Methods}

\section{A. Field of view}

To enable wide field surveillance of the whole oesophagus it is important that the endoscope has sufficient imaging performance across a wide field of view (FOV). In order to measure the FOV we captured 3 images of $1 \mathrm{~mm}$ graph paper at 4 different working distances (WD), 5.7, $8.7,11.7$ and $16.7 \mathrm{~mm}$ (error $\pm 0.3 \mathrm{~mm}$ ). The resulting images are expected to show barrel distortion defined by:

$$
r_{u}=A r_{d}\left(1+k r_{d}^{2}\right)
$$

where $r_{u}$ is the radial distance from the center of the ground truth image to a given vertex $i$ in $\mathrm{mm}, r_{d}$ is the radial distance from the centre of the distorted image to the same vertex $i$ in pixels, $k$ is a constant that describes the magnitude of the distortion and $A$ is a constant used to convert between units of pixels and $\mathrm{mm}$. For each of the 12 images acquired (4 working distances $\times 3$ 
replicates) the position of the centre of the fibre bundle was identified and the radial distance to each vertex in the graph paper, $r_{d}$ (in pixels), was plotted against the true distance, $r_{u}$ (in $\mathrm{mm}$ ), which is known from measuring the physical distance between the markings on the graph paper.

\section{B. Power}

In order for a device to be approved for first-in-human trials, its maximum power must be below the threshold above which thermal damage, photosensitisation and photoallergic reactions may occur [46]. However, the published guidelines for optical radiation exposure limits only concern the retina and skin, and are limited in their application to workers rather than patients, so are not relevant when determining the maximum permissible exposure (MPE) for endoscopic imaging [46, 48] (Translational Barrier 1). Therefore, MPE was established by measuring the maximum broadband power of a clinical endoscope (Olympus Evis Lucera CVL-260SL Xenon light source with an Olympus Gastroscope GIF-FQ260Z, Japan) in normal operating conditions, using a thermal power meter (spectral range 0.19-2.1 $\mu \mathrm{m}$; A-02-D12-BBF-USB, LaserPoint, Italy). Since this standard of care device is approved for clinical use in humans, it can be assumed that this MPE represents safe illumination levels for endoscopic use. The power measurement was repeated for the bimodal endoscope, and the results compared to ensure the determined power of this device was less than the power of the standard of care endoscope.

\section{Resolution}

The resolution of the system is fundamentally limited by the fibre bundle structure. To determine the limiting resolution, images of a 1951 USAF resolution test target (\#53-714, Edmund Optics, USA) were captured at 4 working distances using external illumination from a broadband halogen light source (OSL2B2, Thorlabs, Germany) to reduce specular reflections. Background subtraction was performed using an averaged dark image of 10 frames acquired at the end of the experiment. White light images were analysed since the test target is printed on white reflective photo paper intended for visible imaging. Since both imaging channels are able to resolve the individual fibres of the fibre bundle, the limiting resolution is determined by the fibre bundle properties rather than the properties of the cameras, and so the resolution measured using the white light images is applicable to both imaging channels.

\section{Sensitivity}

For in vivo application, it is important that WGA-IR800 can be detected at low concentrations. The reasons for this are threefold. Firstly, since binding of WGA-IR800 decreases with the progression of the disease, detecting it at low concentrations is important to avoid false positives. Secondly, minimising the amount of any exogenous agent sprayed inside the 
oesophagus is desirable for clinical translation. Thirdly, it is desirable to minimise non-specific binding effects that may become most problematic at high concentrations where they have the potential to reduce contrast. Therefore, determining the sensitivity of the system is a crucial aspect of the characterisation process.

In order to characterise sensitivity, a two-fold dilution series of WGA-IR800 in phosphate buffered saline (PBS) was prepared and $30 \mu \mathrm{L}$ of each solution was pipetted into a well plate ( $\mu$-Slide 18 Well - Flat, ibidi, Germany), which had been spray-painted matte black to avoid specular reflections. Images of the dye were captured at 5 different working distances $(5,10$, 12,16 and $20 \mathrm{~mm}$ ) representative of the range of distances that would be used in vivo. The NIR images were captured using an EM gain of 20 and an exposure time of $200 \mathrm{~ms}$. The images were corrected and coregistered as described in Section 4.4.3. $50 \times 50$ pixel regions of interest (ROIs) were drawn on the white light images inside (signal) and outside (background) of the well. These ROIs were then applied to the coregistered NIR images for analysis in Matlab. The signal-to-noise ratio (SNR) was calculated using:

$$
S N R=\frac{S-B}{\sigma}
$$

where $S$ is the centre of a Gaussian fitted to the pixel intensity distribution in the signal ROI, $B$ is the mean of a Gaussian fitted to the pixel intensity distribution in the background ROI and $\sigma$ is the standard deviation of a Gaussian fitted to the pixel intensity distribution in the background ROI.

The relationship between the SNR and electron multiplying (EM) gain of the NIR sensor was also characterised by capturing images of $30 \mu \mathrm{L}$ of $3100 \mathrm{nM}$ WGA-IR800 while varying the gain at working distances of 5, 9.5 and $12 \mathrm{~mm}$. For an EMCCD, the expected relationship between SNR and gain is:

$$
S N R \propto\left(\text { constant }+\frac{1}{G^{2}}\right)^{-\frac{1}{2}}
$$

where $G$ is the EM gain of the sensor [214]. 


\subsubsection{Results}

\section{A. Field of view}

First, the field of view (FOV) of the system was assessed. An example image displaying barrel distortion can be seen in Figure 4.3. The distortion constant $k$ and the constant $A$ were determined by fitting Eq. 4.1 to the data $\left(\mathrm{R}^{2}=0.9949-0.9974\right)$. The values of $k$ and $A$ were then used to determine the FOV radius $\left(=r_{u}\right)$ based on the radius of the images in pixels $\left(=r_{d}\right)$. Combining these data for the 4 working distances, we determined the angle of the FOV to be $63 \pm 1^{\circ}$, which compares favourably to the manufacturer specified angle of $70^{\circ}$.

\section{B. Power}

To establish a safe maximum power for the endoscope, its maximum broadband power was compared to that of a clinical endoscope. The maximum power from the clinical endoscope in white light reflectance mode was measured to be $19 \pm 1 \mathrm{~mW}$ at a working distance of $1.0 \pm 0.1$ $\mathrm{cm}$ whilst the maximum power from the bimodal endoscope was measured to be $3.9 \pm 0.2 \mathrm{~mW}$ at a working distance of $1.0 \pm 0.1 \mathrm{~cm}$. Hence it was assumed that the bimodal endoscope was well within the safety limits adhered to by current commercial endoscopes.

\section{Resolution}

Resolution was determined by taking images of a USAF test target. At least 4 replicate data points for each line pair element were captured (centred within the FOV) each at working distances of 5, 10, 15 and $20 \mathrm{~mm}$. The Michelson contrast (Eq. 3.17) was calculated for each element (after comb artefact correction) and the results plotted against the reciprocal of the line width of a single line element (Figure 4.4).

A contrast of $1 \%$ that has been reported to be the minimum contrast required for the detection of a pattern across a wide range of targets and conditions [198], but a contrast threshold of 5\% was chosen to avoid effects arising from noise at very low contrast. By finding the intersect of this threshold with exponential fits, the resolution of the bimodal endoscope was determined to be $148 \pm 7,230 \pm 20,320 \pm 40$ and $470 \pm 40 \mu \mathrm{m}$ at working distances of $5,10,15$ and $20 \mathrm{~mm}$ respectively. 
(a)

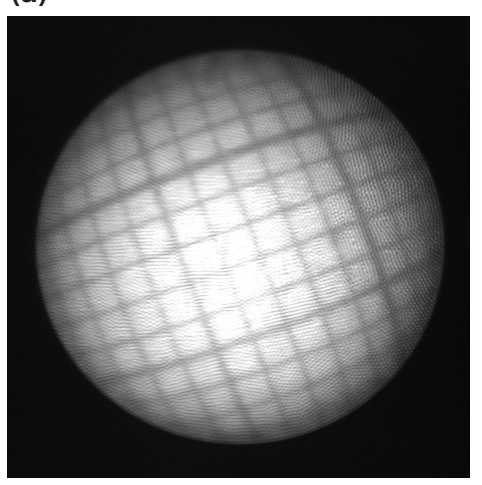

(b)

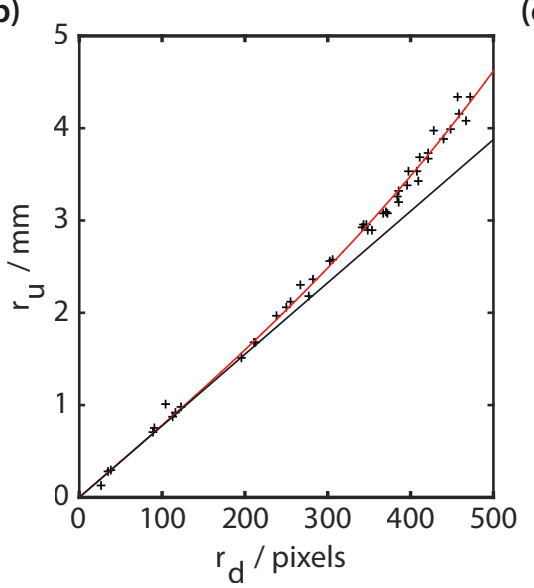

(c)

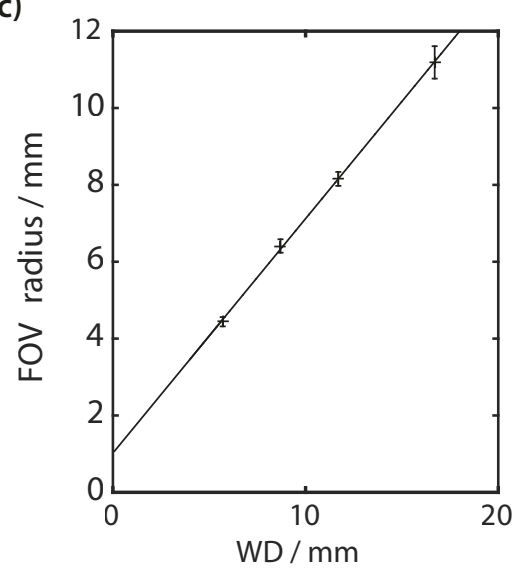

Figure 4.3 Characterisation of the field of view (FOV) of the bimodal endoscope.

(a) An endoscopic image of $1 \mathrm{~mm}$ square graph paper shows barrel distortion. From this image, $r_{u}$ and $r_{d}$ were measured for several vertices on the paper. (b) An example of the fit to Eq. 4.1 for images taken at a working distance (WD) of $5.7 \mathrm{~mm}\left(\mathrm{R}^{2}=0.9954\right)$. The straight line represents the case of no barrel distortion. The fit was used to extract the constant $A$ and the distortion parameter $k$. The values of $A$ and $k$ were used with Eq. 4.1 to determine the FOV radius $\left(=r_{u}\right)$ based on the radius of the images in pixels $(=r d)$. (c) Determined FOV radius for four WDs $\left(\mathrm{R}^{2}=0.9996\right)$. Error bars represent the standard error of the FOV radius derived from the standard errors of the fit parameters $A$ and $k$. From the fitted line, the angular FOV was calculated to be $63 \pm 1^{\circ}$.

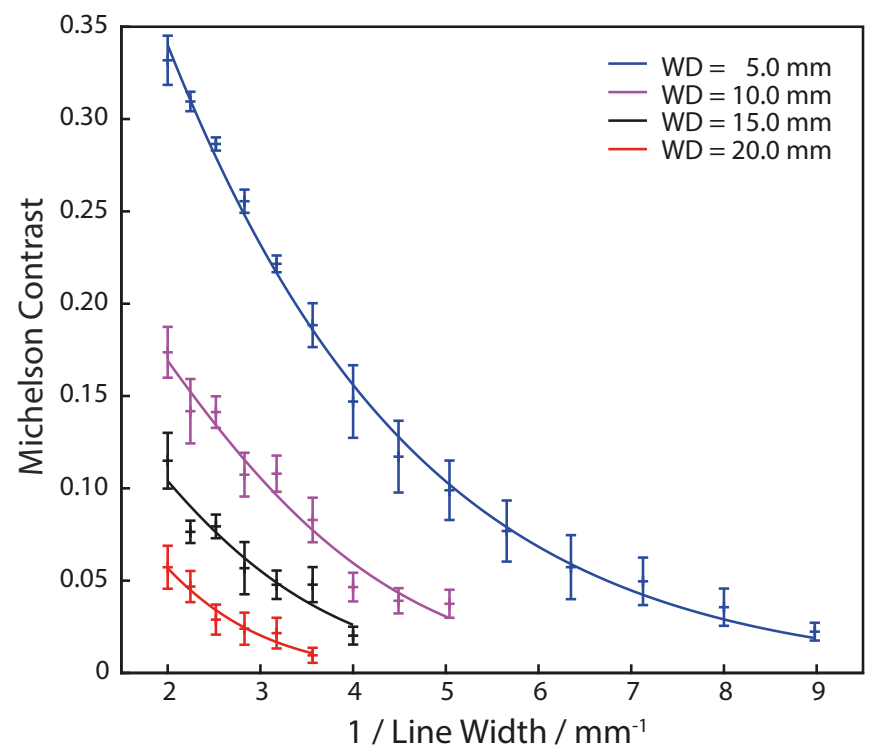

Figure 4.4 Characterisation of the resolution of the bimodal endoscope.

Resolution was characterised by imaging a USAF chart at four different working distances. The resolution was determined as the point where an exponential fit drops below 5\% Michelson contrast. $\mathrm{R}^{2}=0.9965$, $0.9665,0.9068$ and 0.9640 for working distances of $5,10,15$ and $20 \mathrm{~mm}$ respectively. 


\section{Sensitivity}

Finally, the system sensitivity was established by investigating the dependence of signal-tonoise ratio (SNR) on concentration; a SNR of 3 was used to determine the minimum detectable concentration at each working distance. Example images from the white light and NIR channels are shown in Figure 4.5 (a) while Figure 4.5 (b) shows the relationship between SNR and concentration at working distances of 5, 10, and $12 \mathrm{~mm}$. At working distances beyond $16 \mathrm{~mm}$ the dye was not detectable at any concentration $\leq 3100 \mathrm{nM}$. A linear fit is made to these data for SNR $>1$ since below this only noise is expected. For a working distance of $5 \mathrm{~mm}$, the observed non-linear region at high concentration was likely due to saturation, hence was excluded from the linear fit. The minimum detectable concentrations assessed from Figure 4.5 (b) are $110 \pm 60$ $\mathrm{nM}$ and $430 \pm 170 \mathrm{nM}$ for working distances of $5 \mathrm{~mm}$ and $10 \mathrm{~mm}$ respectively. Without comb correction, the minimum detectable concentrations were determined as $170 \pm 40 \mathrm{nM}$ and $810 \pm 520 \mathrm{nM}$ for working distances of 5 and $10 \mathrm{~mm}$ respectively, confirming that removal of the comb artefact grants an improvement in SNR, since it removes low signal pixels representing the cladding between fibrelets (Section 3.4).

The influence of working distance and EMCCD gain on these data was also assessed. The relationship between sensitivity and working distance arises due to the dependence on the irradiance of the excitation light, which decreases with increased WD. This was investigated by capturing images of $30 \mu \mathrm{L}$ of $3100 \mathrm{nM}$ WGA-IR800 at 8 working distances and plotting SNR against working distance (Figure 4.5 (c)). The fit suggests that the SNR falls off as working distance to the power of $-1.91 \pm 0.08$, consistent with the inverse square law expected for an illumination cone. Figure 4.5 (d) shows the fit of the EM gain and SNR data to Eq. 4.3. Increasing EM gain reduces the lifetime of the EMCCD. According to our data, using a gain of $\sim 20$ provides maximises SNR whilst minimising the EM gain required to achieve this SNR, preserving the lifetime of our EMCCD. 
(a)

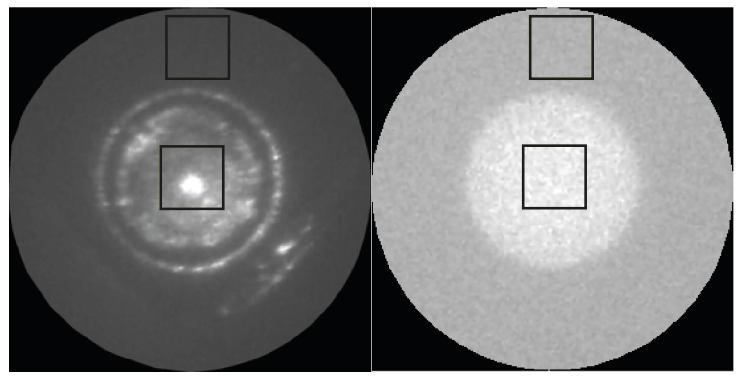

(b)

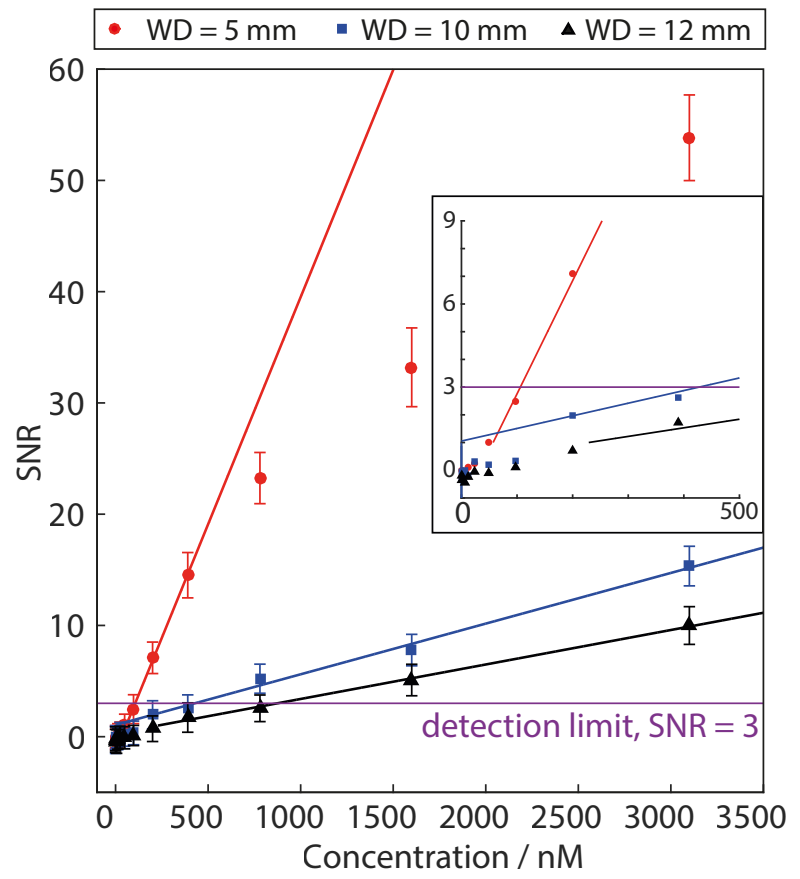

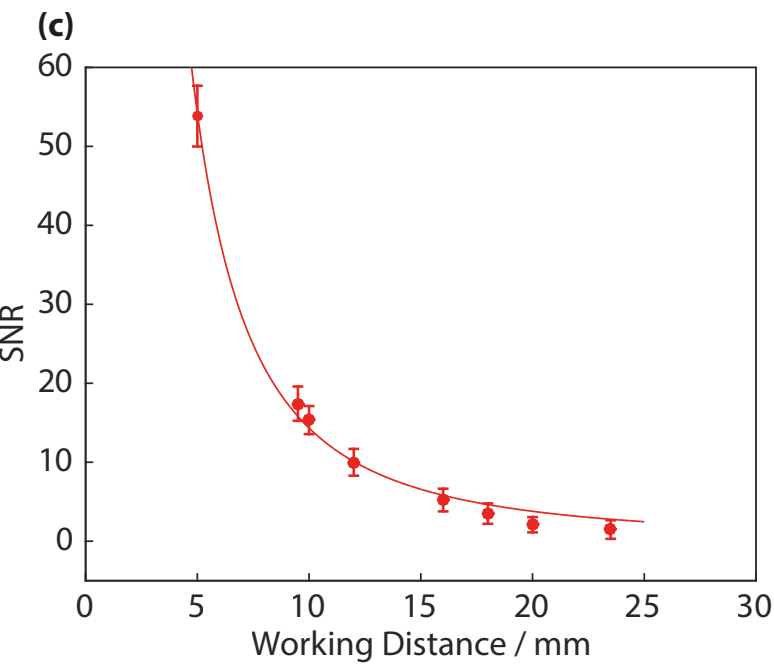

(d)

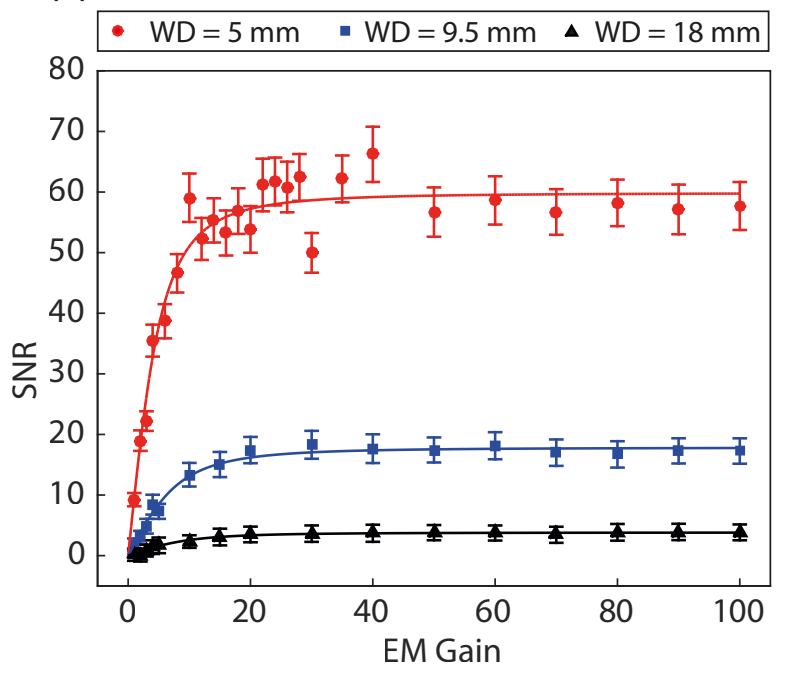

Figure 4.5 Characterisation of the sensitivity of the bimodal endoscope for detection of NIR fluorescence from WGA-IR800.

(a) Coregistered images of white light (left) and NIR fluorescence (right) with example ROIs illustrated for signal and background. (b) The signal to noise ratio (SNR) increases with the concentration of WGAIR800. Linear fits were applied giving $\mathrm{R}^{2}=0.9987,0.9936$ and 0.9974 for working distances of 5,10 and $12 \mathrm{~mm}$ respectively. The horizontal purple line shows the detection limit defined as $\mathrm{SNR}=3$. Inset shows a magnification of the region at low concentration. Error bars have been removed for clarity. (c) SNR of $3100 \mathrm{nM}$ WGA-IR800 follows an inverse power law with the working distance: $\mathrm{R}^{2}=0.9952$. (d) SNR eventually reaches a plateau with increasing EM gain applied to the EMCCD for images taken of 3100 nM WGA-IR800. The lines show the fit according to Eq. 4.3 with $\mathrm{R}^{2}=0.9358,0.9819$ and 0.9727 for working distances of 5, 9.5 and $18 \mathrm{~mm}$ respectively. (Exposure time=200 $\mathrm{ms}$ and EM gain=20 unless otherwise stated. Error bars represent the standard error of the SNR derived from the standard deviation of pixel values in the signal ROI). 


\subsection{Validation Using Biological Samples}

To validate WGA-IR800 imaging with the bimodal NIR fluorescence and WL reflectance endoscope, a range of biological samples were tested (Domain 2, OIB Roadmap, Figure 1.3). These experiments are summarised in Table 4.2.

\section{Table 4.2 Experiments used to validate WGA-IR800 imaging with the bimodal NIR fluorescence and WL reflectance endoscope.}

Gold coloured cells represent gold standards.

\begin{tabular}{|c|c|c|c|c|c|}
\hline Section & Objective & Sample & $\begin{array}{c}\text { Imaging Comparator } \\
1\end{array}$ & $\begin{array}{c}\text { Imaging Comparator } \\
2\end{array}$ & $\begin{array}{l}\text { Pathology } \\
\text { Comparator }\end{array}$ \\
\hline 4.6.1A & $\begin{array}{l}\text { Preliminary validation of the ability of } \\
\text { NIR fluorescence endoscope }+ \text { WGA- } \\
\text { IR } 800 \text { to distinguish between different } \\
\text { tissue types }\end{array}$ & $\begin{array}{l}\text { ex vivo mouse } \\
\text { stomach }\end{array}$ & $\begin{array}{l}\text { NIR fluorescence } \\
\text { endoscope } \\
\text { + WGA-IR800 }\end{array}$ & None & $\begin{array}{l}\text { Squamous and gastric } \\
\text { tissue are } \\
\text { distinguishable by eye }\end{array}$ \\
\hline 4.6.1B & $\begin{array}{l}\text { Validation of the ability of WGA-IR } 800 \\
\text { to distinguish between dysplasia and } \\
\text { Barrett's oesophagus }\end{array}$ & \multirow{3}{*}{$\begin{array}{l}\text { ex vivo } \\
\text { human } \\
\text { endoscopic } \\
\text { mucosal } \\
\text { resection } \\
\text { specimens } \\
\text { (EMRs) }\end{array}$} & $\begin{array}{l}\text { Wide field fluorescence } \\
\text { imaging device } \\
\text { + WGA-IR800 }\end{array}$ & None & $\begin{array}{l}\text { Histopathology of } \\
\text { punch biopsies }\end{array}$ \\
\hline 4.6.1C & $\begin{array}{l}\text { Validation of the accuracy of the NIR } \\
\text { fluorescence endoscope for detection of } \\
\text { WGA-IR } 800\end{array}$ & & $\begin{array}{l}\text { NIR fluorescence } \\
\text { endoscope } \\
+ \text { WGA-IR800 }\end{array}$ & $\begin{array}{l}\text { Wide field fluorescence } \\
\text { imaging device } \\
\text { + WGA-IR800 }\end{array}$ & None \\
\hline 4.6.1C & $\begin{array}{l}\text { Validation of the ability of NIR } \\
\text { fluorescence endoscope }+ \text { WGA-IR } 800 \\
\text { to distinguish between different disease } \\
\text { types }\end{array}$ & & $\begin{array}{l}\text { NIR fluorescence } \\
\text { endoscope } \\
+ \text { WGA-IR800 }\end{array}$ & None & $\begin{array}{l}\text { Histopathology of } \\
\text { entire resection } \\
\text { specimen }\end{array}$ \\
\hline
\end{tabular}

As a preliminary validation of the ability to distinguish between different tissue types using the proposed technique, the novel bimodal endoscope was used to acquire images of healthy ex vivo animal tissue stained with WGA-IR800 (Section 4.6.1A). Mouse stomachs were chosen, as they are readily available, and contain two distinct regions: the upper non-glandular forestomach, which has squamous tissue at the exposed surface, and the lower glandular stomach, which has simple columnar epithelium (gastric type) tissue at the exposed surface. These provide a model of the corresponding tissue types found in the human oesophagus in healthy (squamous) and Barrett's oesophagus (columnar-lined oesophagus) respectively.

In parallel to this work, WGA-IR800 was applied to ex vivo human tissue to ensure that the original results of Bird-Lieberman et al. [200], which validated that WGA-AlexaFluor488 could distinguish between dysplastic tissue and Barrett's oesophagus, were reproducible with WGAIR800 (Section 4.6.1B). Since tissue begins to degrade once it is removed from the body, ex vivo tissue does not usually provide a good model of the contrast expected in vivo (Translational Barrier 4). However, the cell surface sialic acid residues targeted by WGA-IR800 should remain intact for some time after resection [200], so experiments using ex vivo tissue proceeded here. 
Human tissue was taken via endoscopic mucosal resection, a procedure which removes a $\sim 2 \mathrm{~cm}$ diameter section of superficial tissue, called an endoscopic mucosal resection (EMR), from the lining of the oesophagus. A gold standard NIR fluorescence imaging system (Fluobeam-800, Fluoptics, France), which is compact enough to be placed directly in the endoscopy suite, was used to image freshly collected EMRs, and the images were compared to gold standard disease classification from histopathology. The advantage of this on-site method is twofold: first, since the samples are fresh, the degradation of tissue is minimised; second, the ease of the procedure and proximity to available specimens allows a large sample size to be achieved.

Next, the novel endoscope was used to capture images of WGA-IR800 stained EMRs (Section 4.6.1C). To evaluate the accuracy of the novel device for detection of WGA-IR800, the images were compared to those captured using the gold standard imaging device. Finally, images of WGA-IR800 stained EMRs captured with the novel endoscope were compared to gold standard disease classification from histopathology to validate the ability of the technique to distinguish between disease types (Section 4.6.1C).

\subsubsection{Methods}

\section{A. Imaging of mouse stomachs}

To demonstrate the feasibility of imaging WGA-IR800 on a tissue background, and the ability to distinguish between different tissue types, excised mouse stomachs were stained with the fluorescently labelled lectin and images were acquired using the bimodal endoscope. Surplus mice from breeding were obtained post mortem $(n=6)$, so were not classed as regulated procedures under the UK Animals (Scientific Procedures) Act, 1986.

Mouse stomachs were prepared by opening and pinning the excised stomachs on parafilm covered cork. The stomachs were washed with PBS to remove any contents before the first round of imaging. This washing procedure consisted of tilting the stomachs and pouring the PBS such that it ran from left to right over the stomachs to avoid cross contamination between the tissue types (Figure 4.7 (b)). Data acquisition was then performed using EM gain=20 at $\mathrm{WD}=7 \pm 1 \mathrm{~mm}$. After the baseline imaging, $100 \mu \mathrm{L}$ of $29 \mu \mathrm{g} / \mathrm{mL}(780 \mathrm{nM})$ WGA-IR800 was evenly pipetted onto the face of the stomachs and left to incubate for 10 minutes. The stomachs were then imaged four more times: immediately after incubation; after a wash with $5 \mathrm{~mL}$ of PBS; after a further wash of $15 \mathrm{~mL}$ of PBS; and after a final wash of $45 \mathrm{~mL}$ PBS. Each round of imaging captured three images: squamous tissue; gastric tissue; and parafilm covered cork to serve as a background control. For each image, an ROI was drawn in the centre of the image and the mean signal calculated by fitting a Gaussian to the pixel intensity distribution. 
In order to confirm the differential binding of WGA to squamous and gastric tissue types, we repeated the experiment using an additional wash with $33 \mathrm{ml}$ of $1 \mathrm{mM} \mathrm{N}$-acetyl-D-glucosamine (Sigma-Aldrich) in PBS, which has been shown previously to compete with WGA for sialic acid binding [200]. The washing was performed in the same manner as described above. Excess glucosamine that did not run off was left to incubate for 5 minutes before a final wash of $50 \mathrm{~mL}$ of PBS.

\section{B. Imaging of ex vivo human resection specimens with a gold standard imaging device}

Together with our collaborators Massimiliano di Pietro and André Neves, an ex vivo study of 29 EMRs was performed to ensure that the original results of Bird-Lieberman et al. [200], which validated WGA-AlexaFluor488 could distinguish between dysplastic tissue and Barrett's oesophagus, were reproducible with WGA-IR800 [209]. Consecutive patients undergoing endoscopic mucosal resection for Barrett's related early neoplasia were recruited. Freshly collected EMR specimens were washed with $5 \mathrm{~mL}$ of phosphate-buffered saline (PBS), sprayed with $2 \mathrm{~mL}$ of WGA-IR800 $(10 \mu \mathrm{g} / \mathrm{mL})$ in PBS, incubated in the dark for 10 minutes at room temperature, and then washed again with the same buffer, prior to imaging using a wide field intraoperative fluorescence imaging device (Fluobeam-800, Fluoptics, France). A maximum of two punch biopsies ( $2 \mathrm{~mm}$ diameter discs of tissue) were collected ex vivo from each EMR specimen under NIR fluorescence guidance; one from a high intensity region and one from a low intensity region. These were fixed in formalin and classified by a pathologist according to the Vienna classification [215].

\section{Imaging of ex vivo human resection specimens with the bimodal endoscope}

To ensure the accuracy of the bimodal endoscope for detection of WGA-IR800 on human tissue, a pilot study assessing 5 EMRs from a single patient undergoing endoscopic therapy for Barrett's related intramucosal adenocarcinoma was performed. This study was approved by the Cambridgeshire 2 Research Ethics Committee (09/H0308/118).

Resections were first washed with PBS to remove superficial debris and excess mucus then stained with $10 \mu \mathrm{g} / \mathrm{mL}$ (268 $\mathrm{nM}$ ) WGA-IR800 using a small spray bottle to mimic the spray catheter application that would be used in endoscopic practice. Tissues were left to incubate for 10 minutes at room temperature then washed with $15 \mathrm{~mL}$ of PBS to remove unbound fluorescent probe. The EMRs were first imaged with a wide field intraoperative fluorescence imaging device (Fluobeam-800, Fluoptics, France), representing the gold standard for imaging of NIR fluorescence, and then with the bimodal endoscope. 
To fit the entire EMRs ( $\sim 2.5 \mathrm{~cm}$ diameter $)$ into a single field of view, images were captured at a working distance of $\sim 2 \mathrm{~cm}$ using the bimodal endoscope, resulting in a decrease in illumination power density at the sample surface and resulting SNR. To counter this, the exposure time was increased to $2 \mathrm{~s}$, to obtain adequate signal in the images.

In order to compare the endoscope images with the gold standard images, the images were coregistered using the following procedure. First, ROIs were manually drawn around the EMR in the gold standard image and the white light endoscope image (which is already coregistered with the NIR endoscope image as described in Section 4.4.3). Using these ROIs, binary masks were generated, which were then coregistered using the 'imregtform' function in Matlab, which coregisters images based on mutual information. This generated a similarity transformation that was applied to the raw gold standard image to coregister it with the endoscope images. Finally, using both the endoscope mask and the coregistered gold standard mask, the coregistered images were masked so that only image points inside both of the initial ROIs would be compared in the final coregistered images. This process is summarised in Figure 4.6.

After NIR imaging, EMR specimens were fixed in formalin and embedded in paraffin, according to standard histopathological procedures at Cambridge University Hospitals Human Research Tissue Bank. The EMR paraffin block was cut at intervals of $2 \mathrm{~mm}$ and sections were mounted onto glass slides. Slides were H\&E stained and scanned using an Axio Scan.Z1 and imported into Zen 2 lite software (both Carl Zeiss Microscopy, Germany). The EMR sections were scored by the study pathologist every $1 \mathrm{~mm}$ for pathological grade according to the Vienna classification [215], with the help of the ZEN software graphical grid. This allowed construction of a pathology grid, which was superimposed manually onto gold standard fluorescence images. To facilitate histopathological correlation, the normal oesophageal squamous epithelium and oesophageal gastric/intestinal metaplasia (non-dysplastic Barrett's) were grouped together as "non-dysplastic" and any grade of neoplasia, including indefinite for dysplasia, low- and highgrade dysplasia and intramucosal cancer, were grouped together as "dysplastic". After processing the 5 EMR samples and excluding artefacts (including pins, edge effects due to pooled dye under the edges of the tissue and ulcers), it was only possible to perform an accurate co-registration between endoscopic images and histology for one EMR sample. 


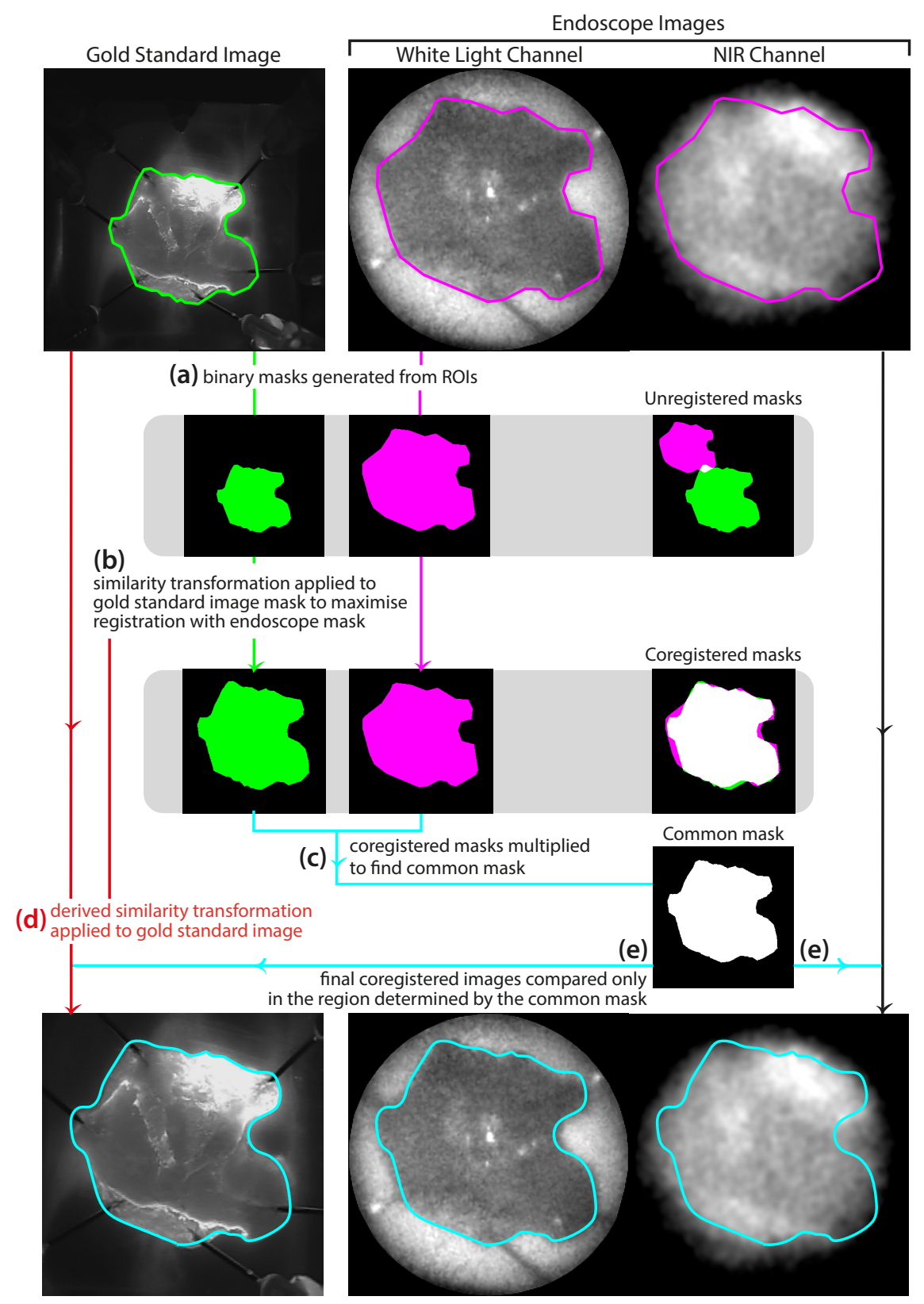

Figure 4.6 Coregistration of NIR fluorescence images of WGA-IR800 binding to human EMRs.

(a). ROIs drawn around the EMRs in the Fluobeam image and white light endoscope image are turned into binary masks. Initially these are unregistered. (b) The Fluobeam mask is similarity transformed in order to maximise overlap with the endoscope mask. (c) The resulting Fluobeam mask and the endoscope mask are multiplied together to find the common mask. (d) The similarity transform found by coregistering the binary masks is now applied to the Fluobeam image to coregister it with the endoscope image. (e) The coregistered images can be compared in the region defined by the common mask, which represents image points that were found inside both of the original unregistered ROIs. 


\subsubsection{Results}

\section{A. Imaging of mouse stomachs}

To demonstrate the feasibility of imaging WGA-IR800 on a tissue background, and the ability to distinguish between different tissue types, excised mouse stomachs were stained with the dye and data was acquired using the bimodal endoscope. The upper non-glandular forestomach, which has squamous tissue at the exposed surface, and the lower glandular stomach, which has simple columnar epithelium (gastric type) tissue at the exposed surface, provide us with a model of the corresponding tissue types found in the human oesophagus in healthy (squamous) and Barrett's oesophagus (columnar-lined oesophagus) respectively. The different regions of tissue within the stomach are indicated in Figure 4.7 (a) and (b).

Gastric tissue was clearly distinguishable ( 2 way ANOVA, $p=0.0005$ ) from the squamous tissue using WGA-IR800 and the bimodal endoscope (Figure 4.7 (c)), even following extensive washing with PBS, suggesting that the WGA-IR800 binds strongly to the gastric mouse tissue. The results shown in Figure 4.7 (d) show a significant reduction of the fluorescence to background level (2-way ANOVA, $\mathrm{p}=0.0472$ ) following the glucosamine wash, confirming the results are due to the differential binding of WGA to different tissue types.

\section{B. Imaging of ex vivo human resection specimens with a gold standard imaging device}

Together with our collaborators Massimiliano di Pietro and André Neves, an intraoperative fluorescence imaging device was used to capture images of WGA-IR800 stained EMRs to validate of the ability of WGA-IR800 to distinguish between dysplasia and Barrett's oesophagus. Mean fluorescence intensity was calculated for areas targeted by punch biopsies. To facilitate histopathological correlation, low- and high-grade dysplasia were grouped together as "dysplastic". Normal squamous and intramucosal cancer biopsies were not included in the analysis.

Dysplastic regions had significantly lower mean fluorescence intensity than biopsies collected from regions with non-dysplastic Barrett's epithelium ( $p<0.001$, two-tailed Wilcoxon matchedpairs signed rank test) (Figure 4.8 (a)). A receiver operating characteristic analysis, with a threshold of mean fluorescence intensity $=0.1115$, indicated an area under the curve (AUC) of $0.84 \pm 0.07$ with a sensitivity of $80 \%$ and a specificity of $82.9 \%$ for dysplasia (Figure 4.8 (b)) [209]. 
(a)

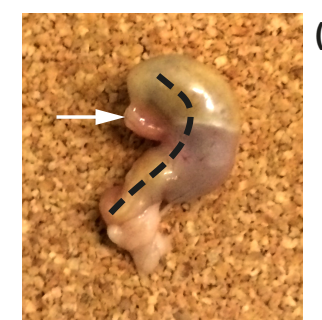

(b)
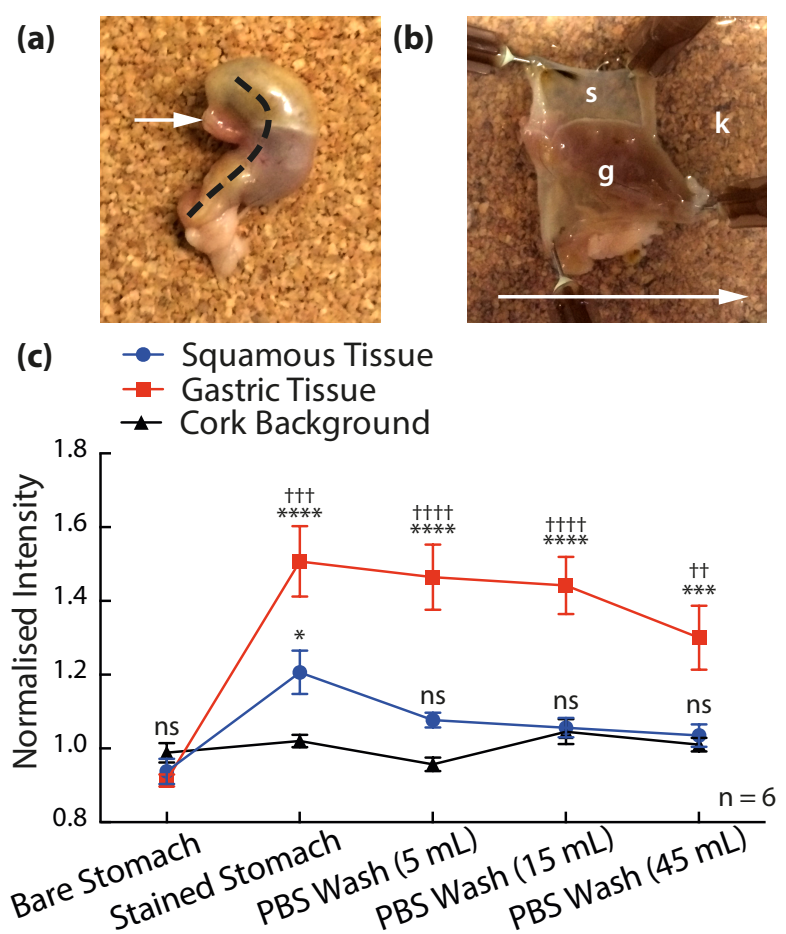

(d) $\square$ Squamous Tissue $\square$ Gastric Tissue $\square$ Cork Background

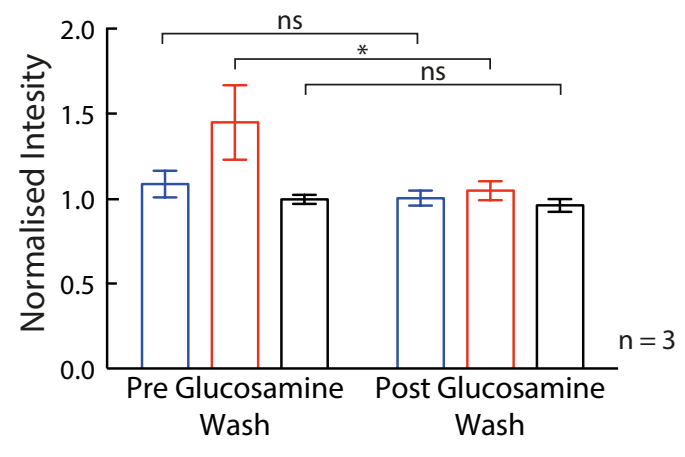

\section{Figure 4.7 WGA-IR800 binding to excised mouse stomach.}

(a), (b). Photographs of the tissue specimens. The arrow in (a) shows the location of the oesophagus. The dotted line shows the approximate location of the cut that was made to open the stomach and expose the inner wall as shown in (b). The arrow in (b) shows the direction of washing. In both images, we can see the limiting ridge separating the upper non-glandular forestomach, which has squamous tissue at the exposed surface, and the lower glandular stomach is evident. For each stomach three images were taken: one at the centre of the squamous tissue (s); one at the centre of the gastric region (g); and one of cork as a background (k). (c) The stomachs were stained with WGA-IR800 and washed with PBS. At each time point, images of each tissue type and cork were taken and the mean intensity in a central ROI of each was calculated. The intensity was normalised to the average background cork level for each mouse. The mean of $n=6$ mice is plotted with error bars representing the standard error in the mean. Statistical testing was carried out using 2-way ANOVA. (ns = no significant difference vs. cork; ${ }^{*} \mathrm{p}<0.05$ vs. cork; ${ }^{* * *} \mathrm{p}<0.001$ vs. cork; ****p $<0.0001$ vs. cork; $\dagger \dagger p<0.01$ vs. squamous; $\dagger \dagger \dagger p<0.001$ vs. squamous; $\dagger \dagger \dagger \dagger p<0.001$ vs. squamous). (d) Following a further wash with glucosamine the bound WGA is removed and the gastric tissue fluorescence intensity returns to the level of the background. Statistical testing was carried out using 2-way ANOVA. (ns $=$ no significant difference; ${ }^{*} \mathrm{p}<0.05$ ).

\section{Imaging of ex vivo human resection specimens with the bimodal endoscope}

To ensure the accuracy of the bimodal endoscope for detection of WGA-IR800, imaging of EMRs collected from a patient with Barrett's oesophagus was performed using the bimodal endoscope and a gold standard imaging system. The coregistered gold standard and endoscope EMR images for all collected specimens are shown in Figure 4.9 (a) and (b) respectively. The high signal observed at the edge of the specimens is due to pooling of the dye between the edge of the tissue specimen and the underlying parafilm, which remains even after washing. The 

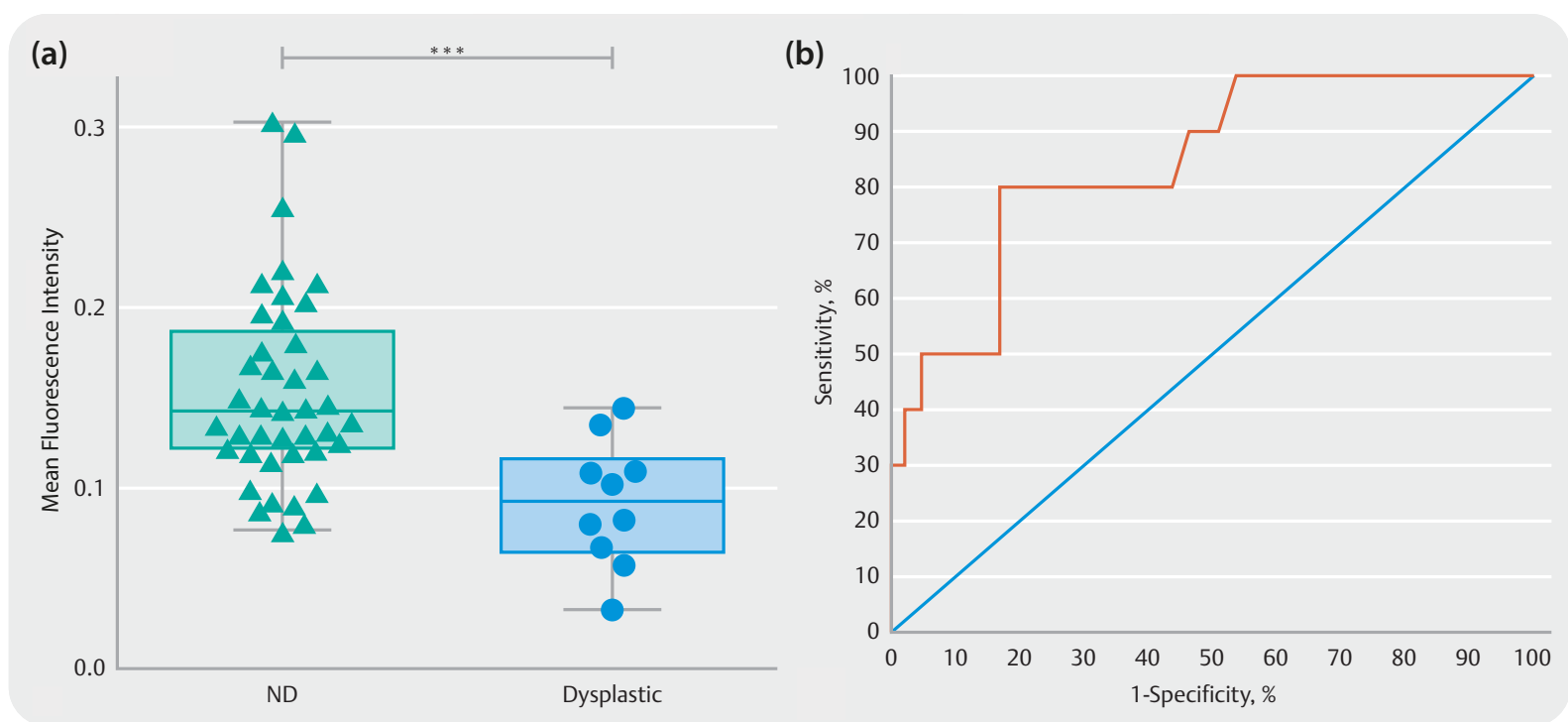

Figure 4.8 NIR fluorescence intensity correlates with dysplasia in punch biopsies.

Consecutive patients $(\mathrm{n}=21)$ undergoing endoscopic mucosal resection (EMR) for Barrett's related early neoplasia were recruited. Freshly collected EMR specimens were sprayed at the bedside with WGA-IR800 and then imaged using an intraoperative fluorescence imaging device (Fluobeam-800, Fluoptics, France). Punch biopsies were collected from each EMR under NIR light guidance. (a) Mean fluorescence intensity for non-dysplastic (ND) punch biopsies is $0.154 \pm 0.054$ vs. $0.092 \pm 0.035$ for dysplastic biopsies. Normal squamous and intramucosal cancer biopsies were not included in the analysis. $n=51$ biopsies, with 10 biopsies $(19.6 \%)$ containing dysplasia. $* * * p<0.001$. (b) Receiver operating characteristic (ROC) for data in (c). Area under the ROC $=0.84 \pm 0.07$; Sensitivity $=80 \%$; Specificity $=82.9 \%$. Reproduced from [209].

intensity recorded in each coregistered pixel in the gold standard and endoscope images was compared to determine if there was correlation between the images and hence whether the data acquired with the endoscope faithfully recapitulates that acquired with the gold standard system.

Intensity scatter plots of these data clearly show a direct relationship between fluorescence intensity in the gold standard and endoscope images for 4 of the 5 EMRs (Figure 4.9 (c)). Spearman's rank correlation coefficient reveals a moderate but significant correlation between the gold standard and endoscope images in these 4 of 5 EMRs $\left(r_{s}=0.90-0.97\right)$, suggesting WGAIR800 NIR fluorescence is accurately measured by our endoscope. The lack of significant correlation in EMR D can be explained by the relatively uniform fluorescence observed in both gold standard and endoscope images (fluorescence signal in central $80 \%$ of EMR within gold standard images: $110 \pm 50,130 \pm 40,160 \pm 40,100 \pm 9,140 \pm 30$ for EMRs A-E respectively). 
EMR A

EMR B

EMR C

EMR D

EMR E

(a) Gold standard: Fluobeam images

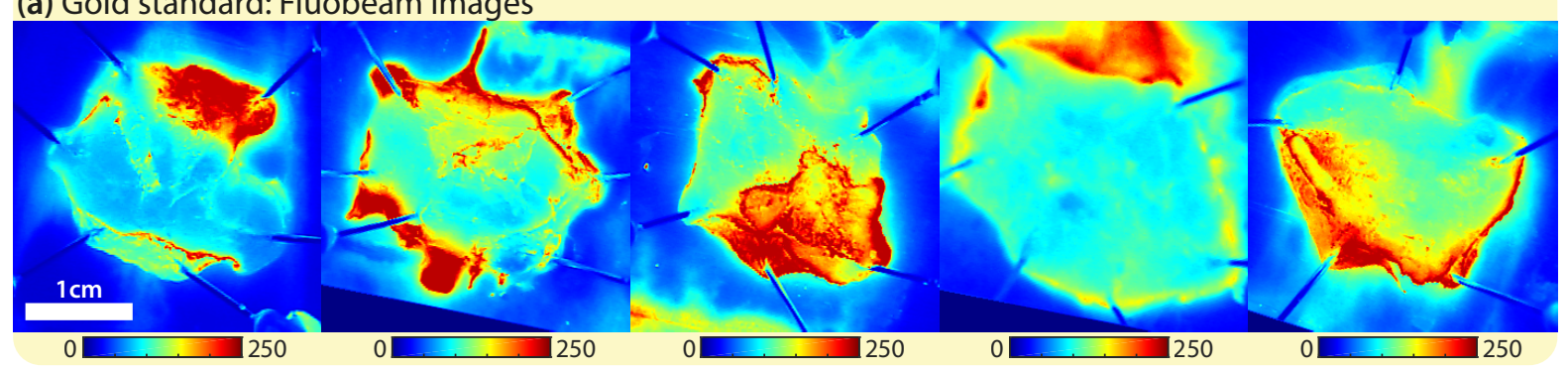

(b) Endoscope images

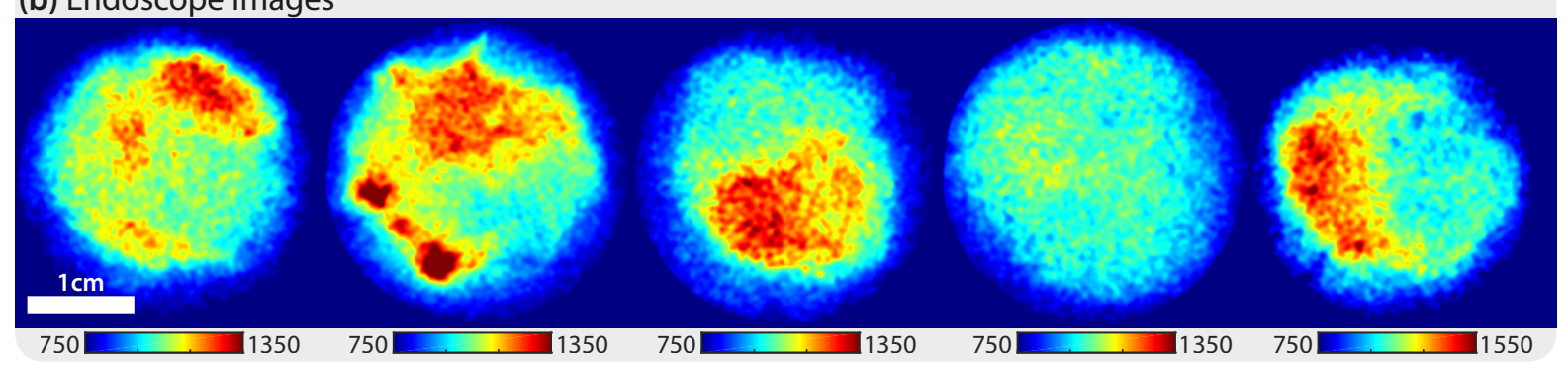

(c) Pixel by pixel comparison

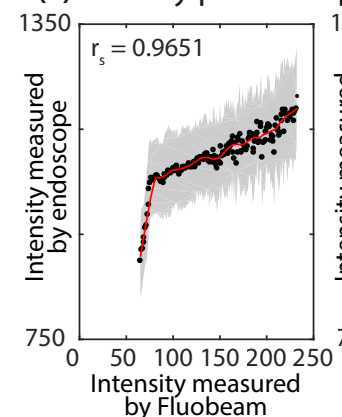

by Fluobeam

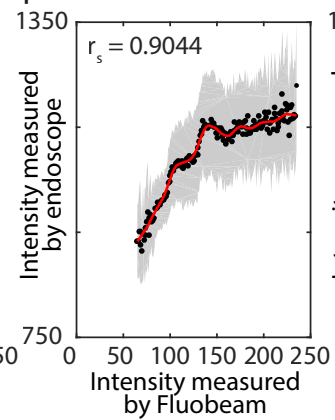

by Fluobeam

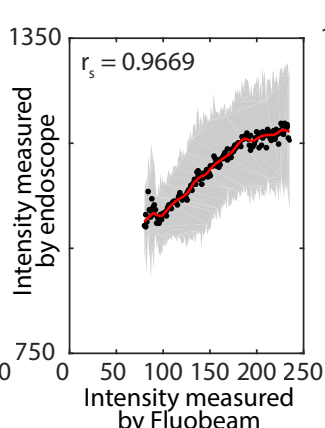

by Fluobeam

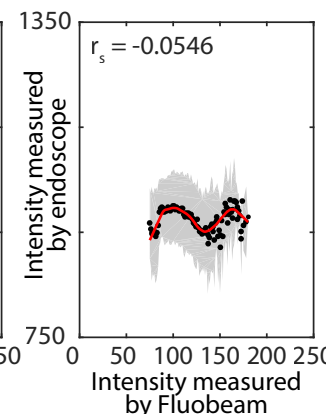

by Fluobeam

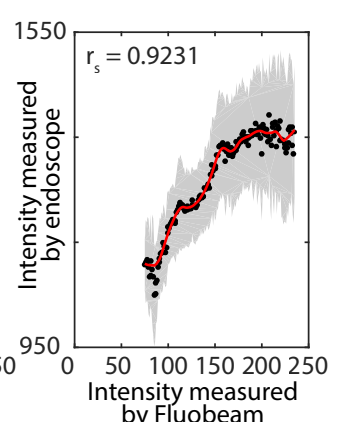

by Fluobeam

\section{Figure 4.9 NIR fluorescence of WGA-IR800 binding to human EMRs captured with the bimodal endoscope and the gold standard imaging device.}

(a). Wide field high resolution NIR images acquired using the gold standard Fluobeam intraoperative imaging system. (b) NIR images acquired using the bimodal endoscope. (c) Correlation between pixel intensities in the gold standard images and the average pixel intensities of coregistered pixels in the endoscope image. The grey areas correspond to the standard deviation of pixels in the endoscope images. A threshold was placed on the gold standard images in order to remove low intensity pixels (due to the signal from the pins holding tissue in place rather than signal from the tissue surface itself) and their corresponding endoscope image pixels. The threshold was determined by removing low intensity Fluobeam pixels until these pixels began to correspond to tissue as well as the pins. The same pixels were then removed from the endoscope images. The thresholds were determined to be $65,65,80,75,75$ for EMRs A-E respectively. The red line shows a robust locally weighted regression smoothing. Spearman correlation coefficients are given within the graphs. High signal intensity observed at the periphery of the specimens is due to pooling of dye between the edge of the tissue specimen and the underlying parafilm. 
Using EMR B, which had two large regions of non-dysplastic and dysplastic tissue, the NIR endoscopy data was manually coregistered to the pathology grid (Figure 4.10 (a)). The expected negative binding relationship between dysplastic and non-dysplastic tissue was confirmed (Figure 4.10 (b)). These results provide a promising preliminary indication that WGA-IR800 fluorescence imaging with the bimodal endoscope is capable of distinguishing between disease pathologies in oesophageal tissue.

(a)

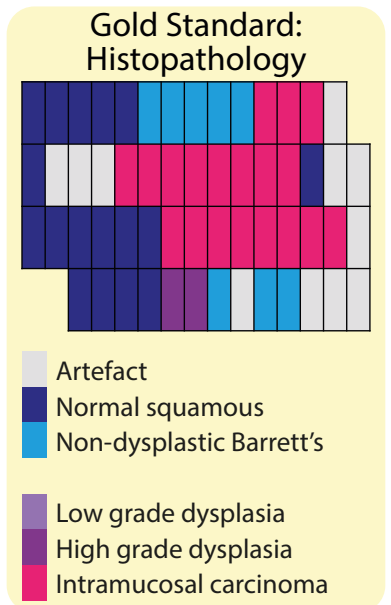

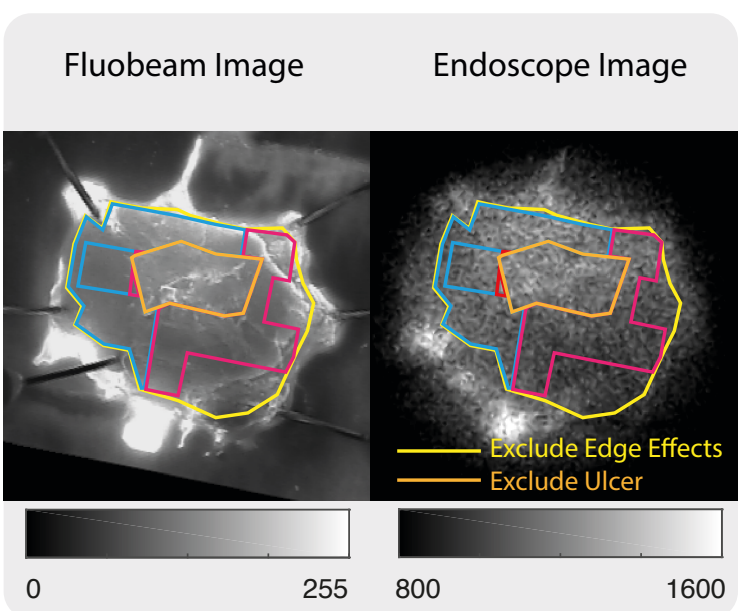

(b)

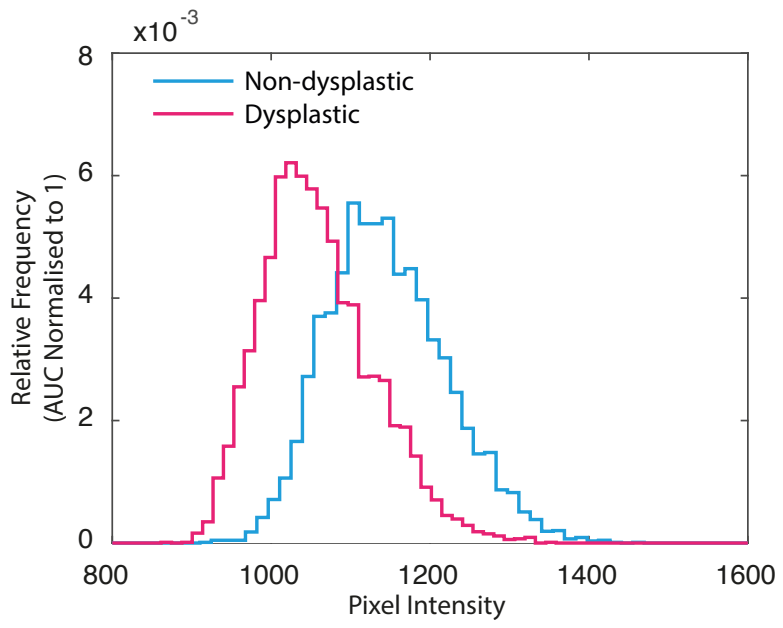

\section{Figure 4.10 Example co-registration with pathology from EMR B.}

Example coregistration with pathology from EMR B. (a) Histology grid manually coregistered to the Fluobeam image and then transferred to NIR endoscope image (since the Fluobeam and endoscope images were previously coregistered). The outside region was excluded to remove edge effects due to pooling of the dye between the edges of the tissue and the parafilm (yellow). An ulcer identified by the pathologist was excluded (orange). Areas labelled "artefact" in the histology grid may: contain pin holes; have no tissue present for analysis (due to the rectangular cuts); or be blurry in the scanned image. (b) To facilitate histopathological correlation, the normal squamous epithelium and oesophageal gastric/intestinal metaplasia (non-dysplastic Barrett's) were grouped together as "non-dysplastic" and any grade of neoplasia, including indefinite for dysplasia, low- and high-grade dysplasia and intramucosal cancer, were grouped together as "dysplastic". Pixel values for the endoscope image are plotted as a histogram for the largest continuous "non-dysplastic" and "dysplastic" regions. 


\subsection{Discussion and Conclusions}

To address the clinical challenge of Barrett's surveillance, a bimodal endoscope capable of acquisition and coregistration of white light reflectance images for endoscopic guidance and of NIR fluorescence images for optical molecular imaging of WGA-IR800, a fluorescently labelled lectin that shows differential binding to Barrett's and dysplastic tissue [200], was designed and built. This endoscope is compact, robust and thus compatible with the clinical environment; it can be used alongside standard of care procedures via the working channel of standard endoscopes; it has safe illumination levels and is based on a CE marked device, facilitating local safety approval for use in humans; and it is able to detect WGA-IR800 at concentrations as low as $110 \pm 60 \mathrm{nM}$ at the shortest working distances, with a field of view of $63 \pm 1^{\circ}$ and an image resolution of $141 \pm 7 \mu \mathrm{m}$.

For preliminary biological validation, the ability of the endoscope to discriminate gastric- from squamous-type tissue in healthy ex vivo mouse stomachs stained with WGA-IR800 was demonstrated $(p<0.001)$. In parallel to this work, the ability to discriminate dysplastic from nondysplastic tissue was confirmed using WGA-IR800 stained human resection specimens and a gold standard NIR fluorescence imaging system $(\mathrm{p}<0.001)$. Finally, the novel bimodal endoscope was compared to a gold standard imaging system for imaging WGA-IR800 stained human resection specimens. The results showed an encouraging correlation between fluorescence signal intensities recorded with the two systems on a per-pixel basis $\left(r_{s}=0.90\right.$ 0.97), with this relating directly to histopathological outcome.

For future ex vivo work, it is necessary to overcome remaining coregistration challenges to enable better comparison of fluorescence data to the gold standard histopathological analysis. Inaccuracies in the coregistration of the pathology grid and fluorescence image may arise for several reasons including: unavoidable deformations and artefacts in the processing of EMRs; the assignment of a single majority pathological grade to a large $1 \times 2 \mathrm{~mm}$ area, which may contain mixed pathologies and cancer field effects; and the manual coregistration of the pathology grid with the fluorescence image (Translational Barrier 3).

To achieve accurate coregistration of single points, punch biopsies can be taken from EMRs. These small ( $\sim 2 \mathrm{~mm}$ diameter) discs of tissue, allow a single point pathology classification to be accurately coregistered with a fluorescence image using the clear hole left by the punch biopsy, which is visible in the fluorescence image (Section 4.6.1B). However, the current approved protocol only allows two punch biopsies to be taken from each EMR, so many EMRs are needed to build a dataset large enough to perform statistical tests (Translational Barrier 3). 
To gather spatial data, the challenge of coregistering pathology grids with fluorescence images remains. In future work, the process of coregistration could be improved by developing a flexible coregistration algorithm. Fiducial markers, such as spots of dye, could provide reference points in both the fluorescence image and histopathological sections. Using these markers, the algorithm could estimate the transformation of the pathology grid due to the nonuniform translation, rotation and stretching of the tissue that occurs during histopathological preparation. However, since EMR histopathology results have a direct impact on the subsequent care of the patient, changes to the histopathological preparation process face challenges in gaining approval.

While the results presented in this chapter are promising, some instrumentation challenges remain for clinical translation. In this work, ex vivo specimens were imaged on a flat surface. Optimal sensitivity in this geometry was found for the shortest WDs in the range that can be expected in vivo. Clinical endoscopy may encompass WDs spanning a range of several centimetres within a single image. To reliably identify dysplasia in these images, it is crucial to distinguish between areas where fluorescence signal is low due to lack of WGA-IR800 binding (true positive for dysplasia), and areas where fluorescence signal is low due to increased WD (false positive for dysplasia). Thus, fluorescence images must be corrected for the variable WD. In addition, a number of developments could be included to ensure that we achieve acceptable SNR at a wider range of WDs. We could employ a more tailored light source, such that more spectral power is deposited across the excitation band of the IR800, or increase the overall illumination power of the system given that we remain significantly below the power used in current commercial endoscopes.

Application in a clinical setting in vivo also requires optimisation of the dye concentration to be used with our system. The sensitivity assays performed here used solutions of the dye in well plates. An important next step would be to calibrate how the sprayed concentration of WGAIR800 relates to the final bound concentration observed on the tissue following application and washing with a spray catheter. A quantitative assessment of the applied concentration of dye, and specific/non-specific binding against a known concentration of sialic acid residues, would allow us to determine the optimum spraying concentration for use in future clinical trials.

Despite these outstanding challenges, the technique has several attractive advantages. WGAIR800 can be applied topically using a spray catheter; requires only 10 minutes incubation time, minimising the disruption to the normal clinical workflow; and is easily displaced by washing with an excess of glucosamine following imaging (Translational Characteristic 1). Our endoscope is robust and compact; provides a coregistered white light image (Translational Characteristic 6); is based around a CE marked accessory channel endoscope, facilitating local 
safety approval for use in humans; is compatible with insertion through the working channel of standard of care endoscopes, enabling easy implementation during standard of care procedures (Translational Characteristic 2); and can be articulated using the familiar standard of care endoscope (Translational Characteristic 3). Fluorescence images are easy to interpret (Translational Characteristic 4), with dysplasia simply represented as low intensity, in contrast to a pattern based OIB, where the interpretation may require complex feature classification (e.g. mucosal and vascular patterns in NBI).

The work presented in this chapter represents progress in translating OMI with WGA-IR800 through Domain 1 and the beginning of Domain 2 of the OIB Roadmap (Figure 1.3). With the majority of the initial validation completed using ex vivo tissue, and the device prepared for application to be locally approved for use in humans, the next step in the roadmap is to perform first-in-human trials.

Much of the groundwork for these trials has already been laid, but in order to ensure the quality and safety of WGA-IR800, it must be synthesised under good manufacturing practice (GMP) conditions before it can be used in humans. Unfortunately, the prohibitively high cost of synthesising a sufficient amount of WGA-IR800 under GMP conditions has thus far prevented the progression of this work to first-in-human trials. Though several avenues were explored to overcome this challenge, including some initially promising academic collaborations, work to find a solution is still ongoing, and could not be achieved within the timescale of the work presented in this thesis.

Still, this delay afforded us an opportunity to address some of the aforementioned instrumentation challenges. One approach is to use multispectral imaging. The ability to acquire spectral information has the potential to allow multiplexed fluorescence imaging of multiple targeted fluorophores. This could be used to increase detection specificity, by enabling the detection of consensus of several targeted molecular imaging probes, or to increase sensitivity by enabling the detection of several molecular imaging probes targeting multiple disease phenotypes. Furthermore, gathering spectral information allows working distance correction, by using data from reflectance detection to normalise the fluorescence signal [145, 216, 217]. Finally, multispectral imaging has the potential to allow delineation of dysplasia based on endogenous contrast alone, circumventing the aforementioned challenges with GMP synthesis of WGA-IR800. 
In the next chapter, the development of multispectral endoscopy is described. The development of this technique for endoscopic detection of multiple fluorescent contrast agents, and for detection of endogenous contrast is described, the latter of which allowed us to make further progress towards clinical translation, carrying out the first-in-human trial of the multispectral endoscope. 



\section{FLEXIBLE ENDOSCOPY: MULTISPECTRAL IMAGING}

This chapter uses material from a proceedings paper by the author entitled: "Spectral band optimization for multispectral fluorescence imaging” (see publications list).

\subsection{Multispectral Fluorescence Imaging of Targeted Fluorescent Molecules}

Multispectral imaging (MSI) enables both spatial (x, y) and spectral (wavelength, $\lambda$ ) information to be recorded. This allows delineation of fluorophores applied during molecular endoscopy [140] based on their spectral properties, rather than a single intensity reading, allowing multiplexed fluorescence imaging of multiple targeted fluorophores $[170,190]$. This could be used to increase detection specificity, by enabling detection of consensus of several targeted molecular imaging probes, or to increase sensitivity to a range of different pathologies by allowing detection of several molecular imaging probes targeting multiple disease phenotypes. It also has the potential to allow control for confounding variables in molecular imaging, for example, by applying an untargeted control dye to account for non-specific binding or non-uniformity of dye application. Furthermore, gathering spectral information allows working distance correction, by using data from diffuse reflectance detection to normalise the fluorescence signal $[145,216,217]$. Given this potential, a multispectral endoscope was developed to achieve multispectral fluorescence imaging (MFI) for application to optical molecular imaging of WGA (Chapter 4). 


\subsection{Multispectral Imaging of Endogenous Contrast}

In addition to its potential for MFI, MSI also has the potential to image endogenous contrast. When it interacts with tissue, light is scattered by endogenous structures such as organelles, cell membranes and cell nuclei and it is absorbed by endogenous chromophores such as melanin and haemoglobin (Figure 1.1). The overall propagation of light through tissue depends on the sizes, shapes, distribution and refractive indices of these scatters and absorbers. Given the complexity of biological tissue, adequately modelling this propagation is complex.

To simplify the model, a common strategy is to treat tissue as a bulk medium with a uniform distribution of scatterers and absorbers. Light propagation is then characterised by a diffusion equation with two wavelength dependent bulk parameters, the absorption coefficient $\mu_{a}(\lambda)$ and the reduced scattering coefficient $\mu_{s}^{\prime}(\lambda)$.

Propagation of broad diffuse illumination through epithelial tissue, as applies to wide field imaging in Barrett's oesophagus, is not well characterised, but models describing the propagation of light from a surface point source have been extensively developed for use in diffuse reflectance spectroscopy (DRS). Furthermore, DRS probes have been deployed in vivo allowing $\mu_{a}(\lambda)$ and $\mu_{s}^{\prime}(\lambda)$ to be derived for oesophageal tissue $\left(\mu_{a}(500 \mathrm{~nm})=0.3-0.7 \mathrm{~mm}^{-1}\right.$ and $\left.\mu_{s}^{\prime}(500 \mathrm{~nm})=1.5-2.1 \mathrm{~mm}^{-1}[169,218-221]\right)$.

At a given wavelength, the values of these coefficients can be used to calculate an optical penetration depth, which is $\sim 1 \mathrm{~mm}$ for green visible light $(550 \mathrm{~nm}), \sim 2 \mathrm{~mm}$ for red visible light (700 nm) and $\sim 3 \mathrm{~mm}$ for NIR light (800 nm) [222], sufficiently deep to image the epithelium $(\sim 0-500 \mu \mathrm{m})$ where dysplastic cells are found. These are consistent with the reported contrast mechanism of narrow band imaging, which uses green light to image the superficial submucosa, approximately 1-2 $\mathrm{mm}$ below the surface, allowing visualisation of submucosal vessels in Barrett's surveillance.

Disease-related biochemical changes in the epithelial layer might alter the distribution and abundance of absorbers and scatterers, resulting in subtle changes in the diffuse reflectance spectrum, which might then be used to reveal the underlying pathology. The potential of this spectral data has been demonstrated in a wide range of potential applications in biomedical imaging $[10,223]$.

MSI can extend the acquisition of spectral image data beyond the current clinically implemented endoscopic methods of autofluorescence imaging and dye-based or virtual chromoendoscopy [224]. In combination with data analysis using spectral unmixing algorithms, MSI has been used to visualise the vascular pattern and the oxygenation status of blood [190, 225-230]; to 
improve detection of gastric [231] and colorectal lesions [232-234]; to identify residual tumour [235]; and to perform tissue segmentation [236, 237].

To capitalise on the potential of this rich endogenous contrast for delineation of dysplasia, in parallel to our work on MFI, a multispectral endoscope for reflectance imaging was developed. We aimed to carry out a first-in-human clinical trial of this device to gain experience operating the device in a real clinical setting, including training of endoscopists in deploying the device through the accessory channel of a standard of care endoscope, to identify improvements required for future in vivo work, and to investigate the potential of multispectral reflectance imaging for delineation of dysplasia in surveillance of Barrett's oesophagus. 


\subsection{Spectrally Resolved Detector Arrays (SRDAs)}

The majority of spectral imaging devices fall into two categories: amplitude division, where the light beam is divided into two new beams; and field division, where the light is filtered or divided based on its position in the beam [238]. Previously reported spectral endoscopy systems use amplitude division, including multiple bandpass filters [233, 239], tuneable filters [232, 240], laser lines [241-243], or detectors dedicated to separate spectral bands [241, 242]. Amplitude division requires the use of multiple expensive optical components, making these systems both bulky, costly and more susceptible to mis-alignment in a clinical environment (Translational Characteristic 2). Furthermore, the sequential acquisition necessary with many of these systems results in slow acquisition rates, unsuitable for real-time clinical imaging.

Spectrally resolved detector arrays (SRDAs) divide the light field using spectral filters deposited directly onto the imaging detector in a mosaic pattern (Figure 3.5). The deposition of filters directly onto the sensor results in a compact and robust device, much more suitable to clinical translation than beam splitting alternatives. Furthermore, in contrast to devices using multiple lenses, mirrors, gratings or detectors, SRDAs require no more alignment than would be needed to implement a monochrome sensor.

Although the mosaic of filters introduces an inherent trade-off between spectral and spatial resolution, since the resolution of fibrescope based imaging is limited by the size of individual fibrelets rather than by the sensor resolution, SRDAs can be implemented in fibrescopic imaging without reducing resolution (Chapter 3). These factors, along with their commercial availability, low cost and acquisition speed, underline SRDAs as a highly suitable solution for multispectral endoscopic imaging (Translational Characteristic 2) [189, 190], motivating the development of an SRDA-based multispectral endoscope.

For the remainder of this chapter, the preliminary work on the development of SRDA-based multispectral endoscopy is described. The design, characterisation and preliminary validation of a clinically translatable SRDA-based multispectral endoscope with two alternative optical configurations, one for fluorescence imaging of fluorescent contrast agents, and one for reflectance imaging of endogenous tissue contrast (Section 5.4) is presented. The feasibility of using this device to perform multispectral fluorescence imaging (MFI) is assessed in Section 5.5 using ex vivo models. Following this, multispectral reflectance imaging is investigated in a first-in-human trial in Section 5.6. 


\subsection{Materials and Methods}

\subsubsection{Fluorescent Contrast Agents}

The main disadvantage of SRDAs is their lower sensitivity compared to EMCCDs such as that used in Chapter 4, so the fluorescence signal is particularly important. We therefore decided to use AlexaFluor-647 (AF647, Thermo Fisher Scientific, USA) for this work, as it exhibits significantly brighter fluorescence than IR800. Furthermore, high powered LED sources are more readily available in the far-red than in the NIR, allowing additional power to be deposited across the excitation peak of AF647, further increasing the fluorescence signal. The AlexaFluor series of dyes are also available with fluorescence emission peaks at a range of closely spaced wavelengths, so multiplexed imaging of two similar fluorophores can be assessed. Additionally, AF647 is commercially available as a WGA conjugate, WGA-AF647 (Thermo Fisher Scientific, USA). It was hoped that this would improve our chances of achieving translation to first-in-human trials.

\subsubsection{Endoscope Design}

A multispectral endoscope based around the PolyScope accessory channel endoscope (Section 3.2) and a compact SRDA (CMS-V, SILIOS, France) was designed as shown in Figure 5.2. The SRDA consists of 9 spectral filters ( 8 narrow bands; average FWHM $30 \mathrm{~nm}$; centre wavelengths $553,587,629,665,714,749,791,829 \mathrm{~nm} ; 1$ broad band; $500-850 \mathrm{~nm}$ ), deposited as a $3 \times 3$ super-pixel across a CMOS sensor (NIR Ruby sensor, UI1242LE-NIR, IDS, square pixel size $5.3 \mu \mathrm{m})$. Due to the compact SRDA, the system is much smaller than the bimodal endoscope (Section 4.4.2) as shown in Figure 5.1. The optics are securely housed inside the same portable light tight enclosure described in Section 4.4.2. 
(a)

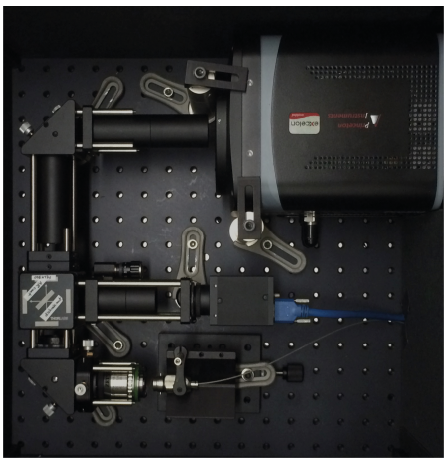

(b)

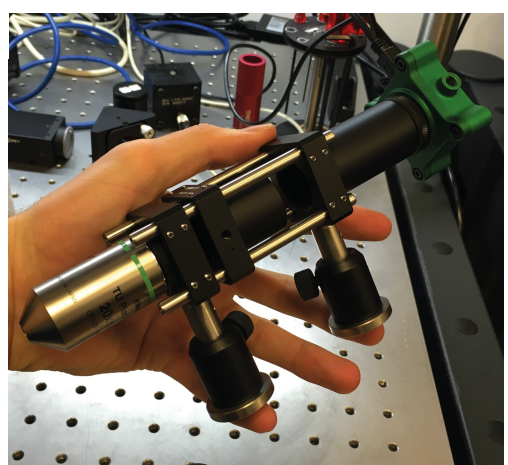

Figure 5.1 SRDA-based endoscope compared to bimodal NIR fluorescence and WL reflectance endoscope.

The SRDA-based multispectral endoscope (b) is more compact than the bimodal NIR fluorescence and WL reflectance endoscope (a) described in Chapter 4 as it requires fewer optical components and utilises a single compact SRDA.

The device has two alternative optical configurations.

- For fluorescence imaging (Figure 5.2 (a)), illumination is provided by a narrow band ultrahigh power LED (Figure 5.3) (UHP-T-LED-635-EP, Prizmatix, Israel) coupled into the PolyScope illumination channel using an achromatic doublet lens (AC254-030-A, Thorlabs, Germany) housed inside a custom coupler with a smooth bore for the PolyScope illumination fibre tip. An objective lens (NA=0.5, UPLFLN20x, Olympus, Japan) and an achromatic doublet lens ( $f=100 \mathrm{~mm}$, ACA254-100-A, Thorlabs, Germany), focus light from the 10,000-fibrelet bundle onto a compact SRDA (CMS-V, SILIOS, France) allowing multispectral fluorescence imaging.

- For reflectance imaging (Figure 5.2 (b)), the illumination is replaced with a broadband UHPLED (Figure 5.3) (T7359, Prizmatix, Israel). A 90:10 beam splitter (BSN10R, Thorlabs, Germany) relays $10 \%$ of the light, through an achromatic triplet lens $(f=40 \mathrm{~mm}$, TRH254040-A-ML, Thorlabs, Germany), into a $1000 \mu \mathrm{m}$ fibre (M35L01, Thorlabs, Germany) coupled to a spectrometer (AvaSpec-ULS2048, Avantes, Netherlands). This was used to acquire a single gold standard reflectance measurement of the entire image area during clinical imaging. 
(a) Fluorescence detection
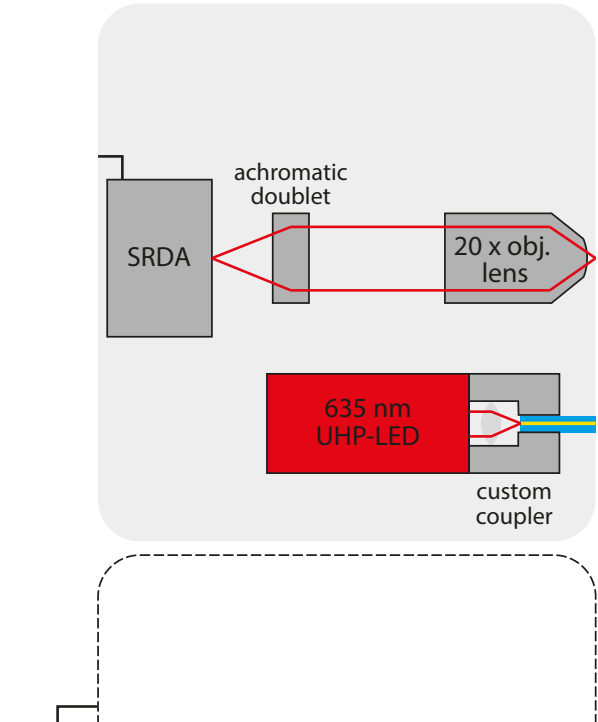

(a) Fluorescence or (b) Reflectance (b) Reflectance detection

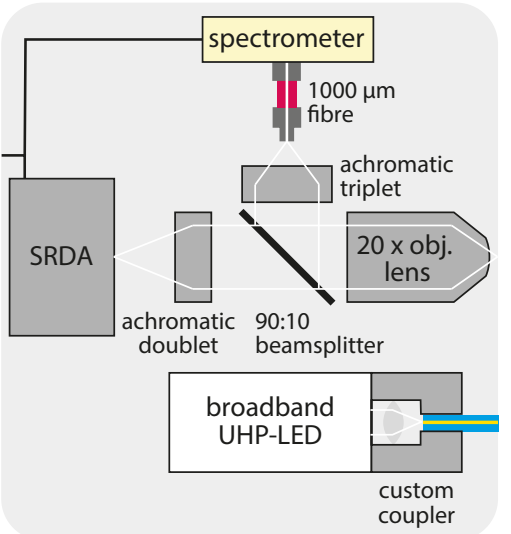

sterile region for direct patient contact
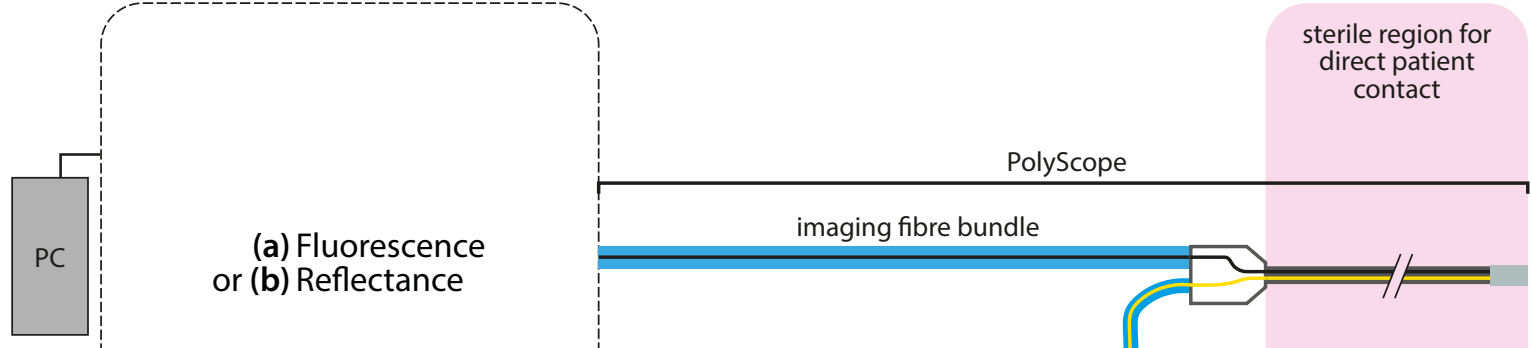

PolyScope

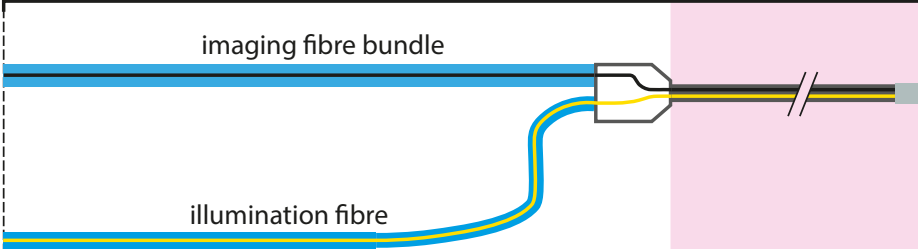

back end optics

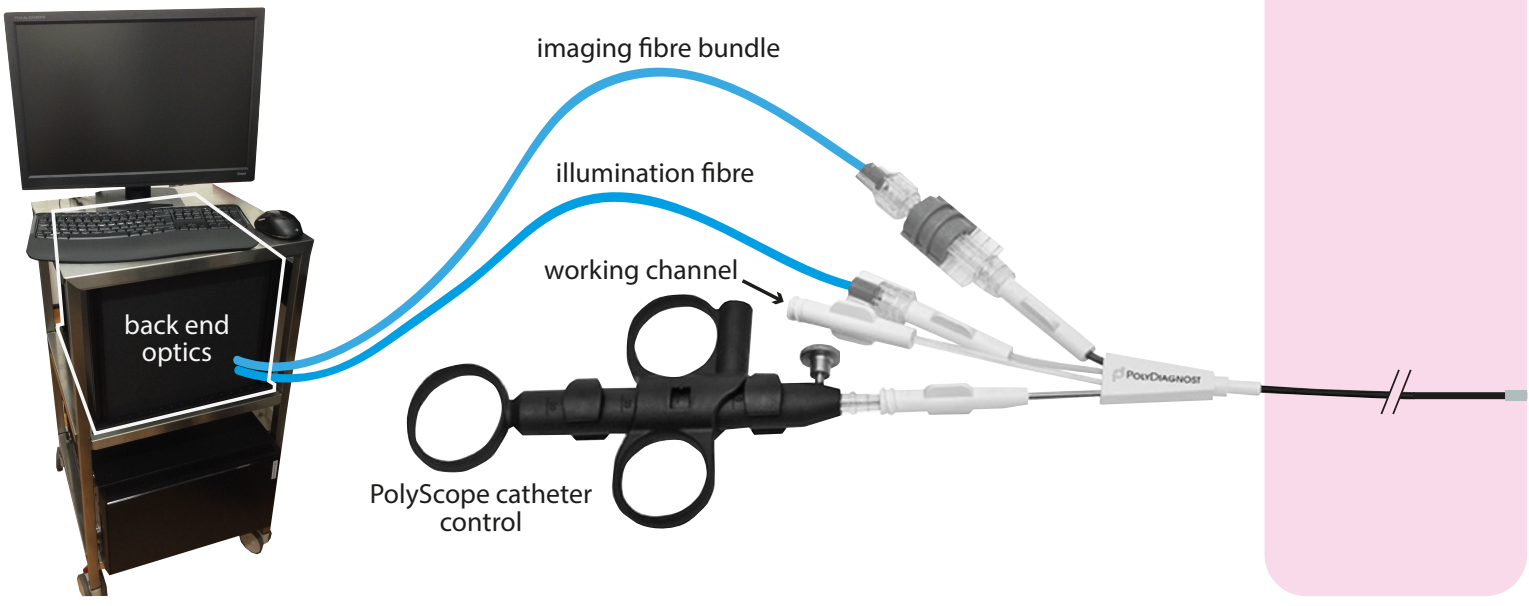

\section{Figure 5.2 SRDA-based multispectral endoscope.}

The system is based around the PolyScope accessory channel endoscope (PolyDiagnost, Germany). The device has two alternative optical configurations. (a) For fluorescence imaging, illumination is provided by a narrow band ultra-high power LED (UHP-T-LED-635-EP, Prizmatix, Israel) coupled into the PolyScope illumination channel using a custom coupler. An objective lens (NA=0.5, UPLFLN20x, Olympus, Japan) and an achromatic doublet lens ( $f=100 \mathrm{~mm}$, ACA254-100-A, Thorlabs, Germany), focus light from the 10,000fibrelet bundle onto a compact SRDA (CMS-V, SILIOS, France) allowing multispectral fluorescence imaging. (b) For reflectance imaging, the illumination is replaced with a broadband UHP-LED (T7359, Prizmatix, Israel). A 90:10 beam splitter (BSN10R, Thorlabs, Germany) relays 10\% of the light, through an achromatic triplet lens ( $f=40 \mathrm{~mm}$, TRH254-040-A-ML, Thorlabs, Germany), into a $1000 \mu \mathrm{m}$ fibre (M35L01, Thorlabs, Germany) coupled to a spectrometer (AvaSpec-ULS2048, Avantes, Netherlands), to acquire a single gold standard reflectance measurement of the entire image field. The pink area of the PolyScope catheter must remain sterile once unpacked, as this part is in direct contact with the patient during endoscopy. 

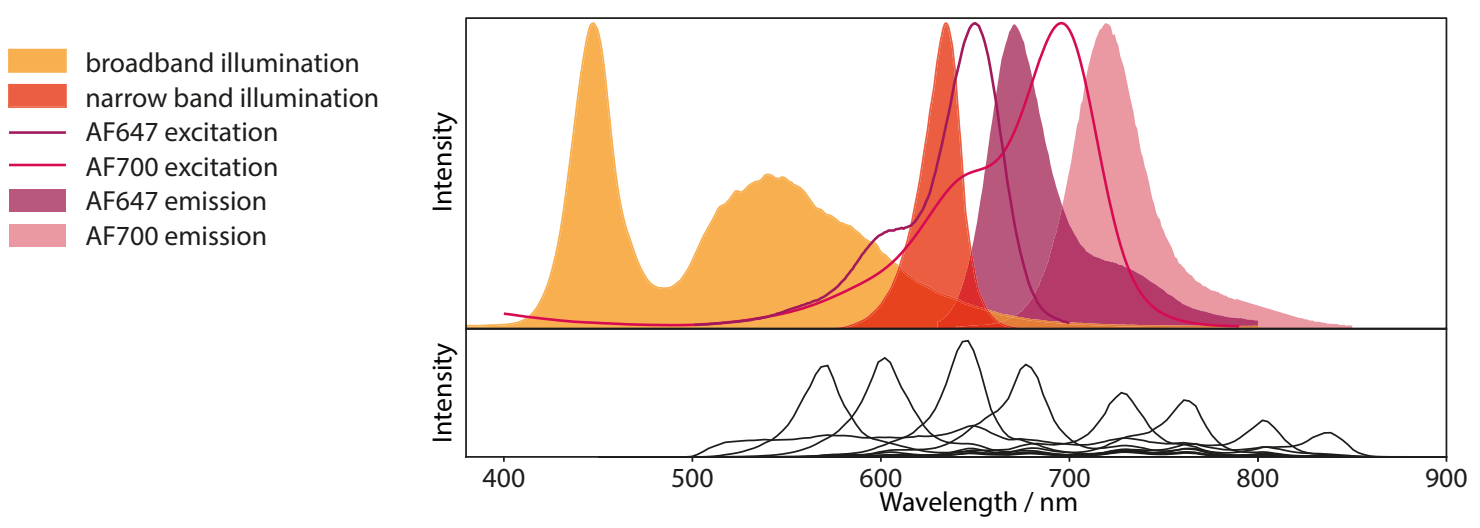

Figure 5.3 Spectra of the components and contrast agents used for multispectral imaging.

The spectra of the light sources used for illumination in MFI and multispectral reflectance imaging, narrow band UHP-LED and broadband UHP-LED respectively (data from Prizmatix). Excitation and emission spectra of AF647 and AF700 are shown (data from ThermoFisher [244]). The spectral response of the multispectral endoscope is shown below for reference (data from Section 5.5.1C).

\subsubsection{Image Acquisition and Image Corrections}

For the non-clinical fluorescence system (Figure 5.2 (a)), images were captured in uEye Cockpit (IDS, Germany) and saved as 8-bit BMP files. Videos were captured in uEye Cockpit (IDS, Germany) and saved as AVI files. Data analysis was carried out using Matlab® (MathWorks, USA). For the clinical reflectance system (Figure 5.2 (b)), a LabVIEW (National Instruments, USA) Visual Interface (VI) was developed to capture and display the images and spectra for the endoscopist to view them in real-time. The raw images captured by the SRDA were demosaicked and decombed using the interpolation methods outlined in Section 3.3.3. For the clinical system, a false colour RGB image was generated by assigning the narrow bands centred at 629,587 and $553 \mathrm{~nm}$ to $\mathrm{RGB}$ channels respectively.

\subsubsection{Spectral Unmixing}

For each spatial $(\mathrm{x}, \mathrm{y})$ position in a multispectral image, spectral (wavelength, $\lambda$ ) information is acquired. Each raw spectrum is a sum of reflection and fluorescence from any fluorophores present, those exogenously applied or those endogenous to tissue. To make use of the spectral information, "unmixing" the individual spectra, or "endmembers", from each of these contributions is of interest. In MFI, these endmembers are the reflected illumination spectrum and the fluorescence emission spectrum of each fluorophore. In reflectance imaging of endogenous tissue contrast, the endmembers might be the spectra of individual disease 
phenotypes or the individual reflection spectra of diagnostically relevant biological molecules. To extract the abundance of each endmember from the raw spectrum, it is necessary to perform spectral unmixing. In this work non-linear least squares unmixing was used to fit linear sums of modelled endmembers to the raw spectra captured by the endoscope.

\subsection{Multispectral fluorescence imaging (MFI)}

After the SRDA-based fluorescence endoscope was designed and built, characterisation was carried out with respect to the safety of the system, by quantifying its illumination power (Section 5.5.1A) and with respect to its intended operation, by quantifying its resolution(Section 5.5.1B), FOV (Section 5.5.1B) and spectral response (Section 5.5.1C). Following this, the device was validated for MFI, first using a dilution series of AF647 (Section 5.5.1D), and then using fluorescent phantoms containing AF647 and AF700 placed in a whole ex vivo pig oesophagus, simulating clinical endoscopy (Section 5.5.1E).

\subsubsection{Methods}

\section{A. Power}

As previously discussed in Section 4.5.1B, defining a maximum permissible exposure for in vivo optical imaging is difficult due to a lack of published safety standards. Thus, to establish a safe maximum power for the multispectral endoscope, its maximum broadband power was compared to that of a clinically approved standard of care endoscope as described in Section 4.5.1B.

\section{B. Resolution and FOV}

The resolution of the device was determined as described in Section 3.3.5A. The FOV is determined by the PolyScope accessory channel endoscope, so the FOV of the multispectral endoscope is the same as that measured for the bimodal endoscope in Section 4.5.1A. 


\section{Spectral response}

In order to model endmembers for spectral unmixing, "ground truth" spectra of interest must be propagated through the spectral response of the multispectral endoscope, so it is important that this is characterised. To characterise the spectral response of the multispectral endoscope, a broadband halogen light source (OSL2BIR, Thorlabs, Germany) was fibre coupled into a monochromator (CM110, AG1200-00500-303 grating, blaze wavelength 500nm, grating density $1200 \mathrm{~mm}^{-1}$, Spectral Products, USA), which was used to increment the wavelength between $450-900 \mathrm{~nm}$ (with a step size of $3 \mathrm{~nm}$ and a FWHM of $\sim 2 \mathrm{~nm}$ ). The resulting narrow bands of illumination were directed into the detection pathway of the multispectral endoscope (Figure 5.4 (a)). At each wavelength step, the SRDA captured 10 images.

Subsequently, the detection arm of the multispectral endoscope was replaced with a spectrometer (AvaSpec-ULS2048, Avantes, Netherlands) (Figure 5.4 (b)), and the monochromator was scanned again; an illumination spectrum was acquired at each scan wavelength. For each scan wavelength, a dark image was subtracted from each of the 10 multispectral images. The mean pixel intensity across the ten images was calculated and this value was divided by the peak intensity of the illumination spectrum to yield the final spectral response. This calculation was performed at each scan wavelength to yield the spectral response curves.

\section{Multispectral fluorescence imaging of AF647 in solution}

To validate the performance of the multispectral fluorescence endoscope in MFI, a dilution series of AF647 (30 $\mu \mathrm{L}$ of a 1:2 dilution from $1 \mathrm{mg} / \mathrm{mL}-0.0039 \mathrm{mg} / \mathrm{mL}$ ) was prepared in a clear well plate (ibidi, Germany). Images of the dilution series were captured using the multispectral fluorescence endoscope (Figure 5.5 (a)). As a gold standard comparison, the detection pathway of our endoscope was replaced with a spectrometer (AvaSpec-ULS2048, Avantes, Netherlands) to measure the 'ground truth' spectrum from each concentration of dye (Figure 5.5 (b)). This was repeated 5 times with removal and replacement of the sample. The average spectrum was calculated from the 5 repeats for each concentration.

\section{E. Multispectral fluorescence imaging of multiple fluorophores in ex vivo pig oesophagus}

After testing the ability of the multispectral fluorescence endoscope to detect a single fluorophore by unmixing fluorescence and reflectance endmembers in images of an AF647 dilution series, the ability of the endoscope to image multiple fluorophores in a more realistic endoscopic scenario was tested. A clinical endoscopic setting was simulated using a whole ex vivo porcine oesophagus (Figure 5.6 (a)) (Med Meat Supplies, UK). For fluorescence targets, 


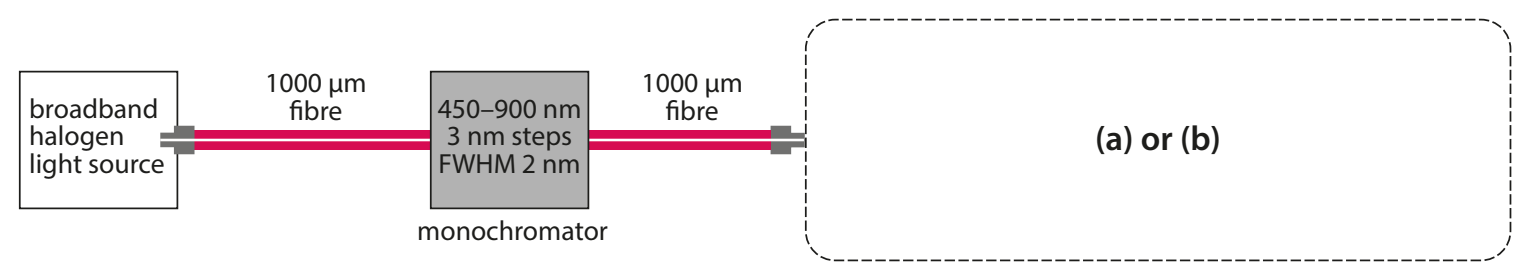

(a) Multispectral endoscope detection arm

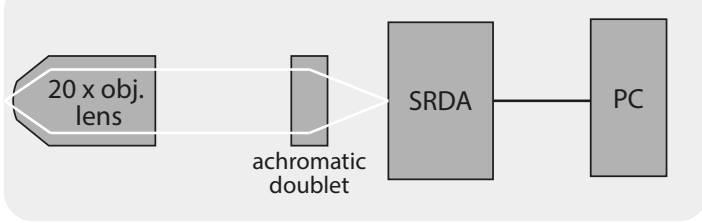

(b) Gold Standard: Spectrometer

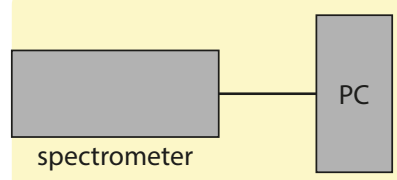

Figure 5.4 Spectral response characterisation equipment.

A broadband halogen light source (OSL2BIR, Thorlabs, Germany) was fibre coupled into a monochromator (CM110 Compact 1/8 Monochromator, AG1200-00500-303 grating, blaze wavelength $500 \mathrm{~nm}$, grating density $1200 \mathrm{~mm}^{-1}$, Spectral Products USA), which was used to increment the wavelength between $450-900 \mathrm{~nm}$ (with a step size of $3 \mathrm{~nm}$ and a FWHM of $\sim 2 \mathrm{~nm}$ ). This was directed into (a) the multispectral endoscope and (b) a spectrometer (AvaSpec-ULS2048, Avantes, Netherlands).

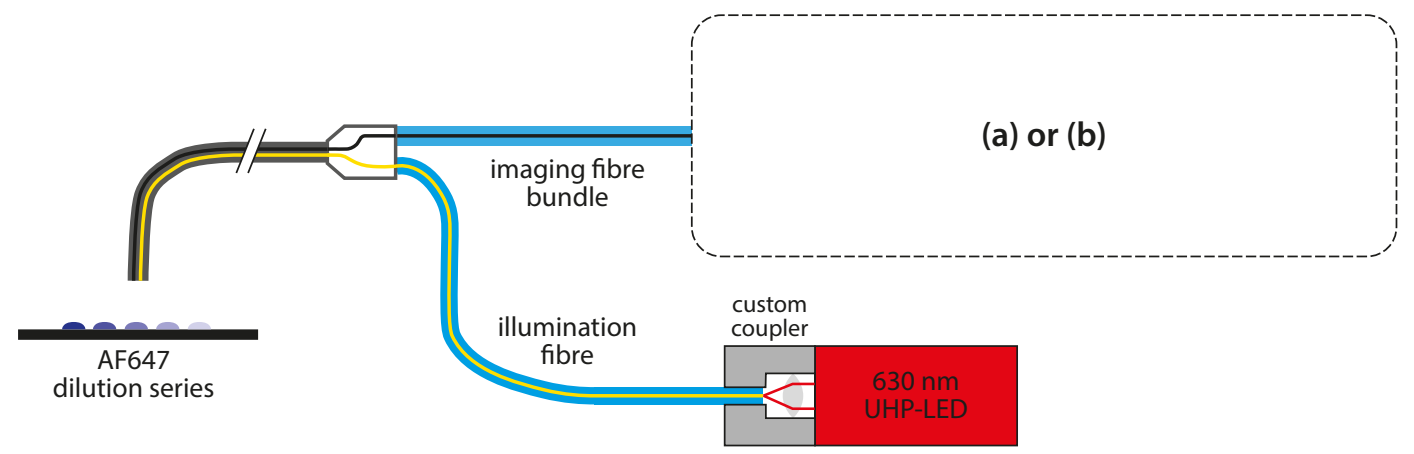

(a) Multispectral endoscope detection arm

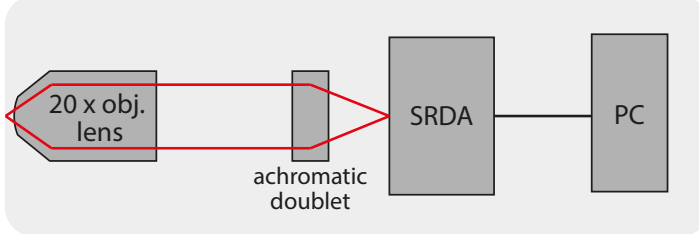

(b) Gold Standard: Spectrometer

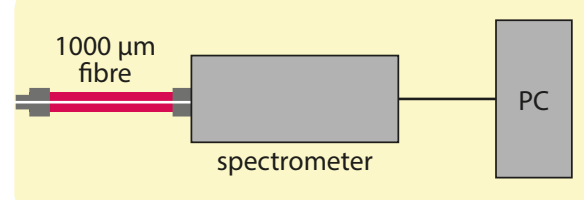

Figure 5.5 Fluorescence detection characterisation equipment.

(a) The detection arm of the multispectral fluorescence endoscope is used to capture images of a 2:1 dilution series of Alexa-Fluor-647 in a well plate (ibidi, Germany). (b) Immediately following this, the multispectral endoscope detection pathway is replaced by a spectrometer (AvaSpec-ULS2048, Avantes, Netherlands) to measure the AF647 spectra. These spectra can be used to determine the ground truth concentration. 
agarose tissue mimicking phantoms containing AlexaFluor 647 (AF647), AlexaFluor 700 (AF700) and PBS as a control were prepared (Figure 5.6 (b)).

Briefly, the phantoms were prepared by mixing equal parts of a $6.0 \%$ agar solution with $160 \mu \mathrm{M}$ fluorescent dye dilutions in PBS. The liquid agarose was pipetted onto glass slides to cool and set to form $\sim 5 \mathrm{~mm}$ diameter, $\sim 3 \mathrm{~mm}$ thick droplets. A trained endoscopist (Massimiliano di Pietro) used endoscopic forceps via the working channel of a clinical endoscope (Figure 5.6 (c)) (GIF-H260, Olympus, Japan), to carefully place these phantoms inside the ex vivo porcine oesophagus, where they adhered to the luminal surface by surface tension (Figure 5.6 (d)).

Next, the multispectral endoscope was taped to the side of the clinical endoscope since the working channel of the endoscope in use was only $2.8 \mathrm{~mm}$, too small for the diameter of the 3 mm PolyScope catheter. Video was recorded at $\sim 5$ frames per second as the endoscope and PolyScope were slowly moved into the oesophagus and then slowly withdrawn.

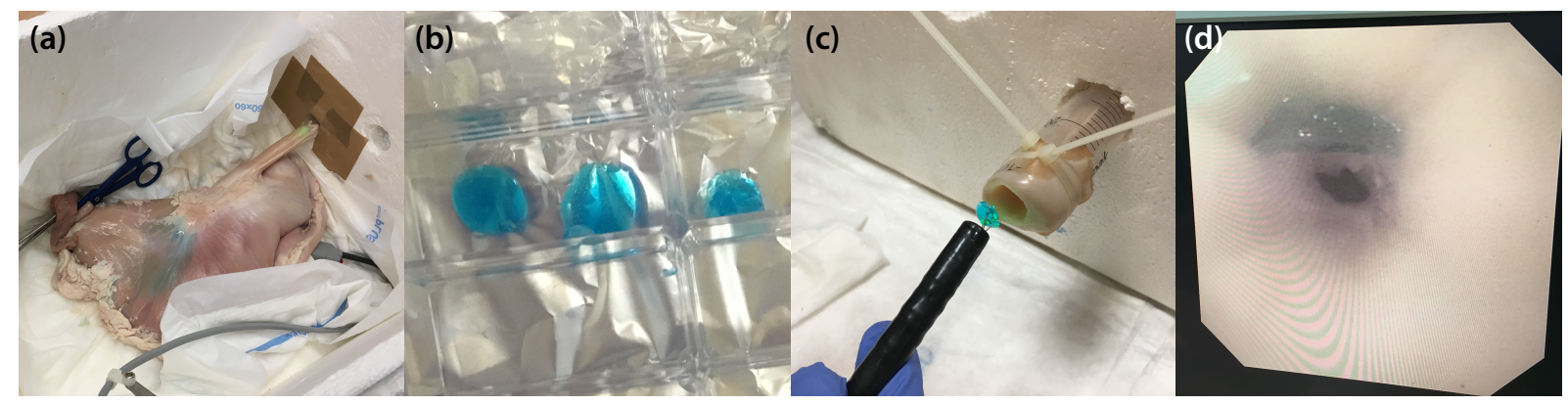

Figure 5.6 Procedure for preparing ex vivo porcine oesophagus for endoscopic imaging.

(a) Whole ex vivo porcine oesophagus. (b) Agarose tissue mimicking phantoms containing PBS, AF647 and AF700 were prepared. (c) The agarose phantoms were inserted inside the ex vivo porcine oesophagus using endoscopic forceps. (d) The phantoms adhered to the luminal surface of the oesophagus by surface tension.

\subsubsection{Results}

\section{A. Power}

To establish a safe maximum power for our multispectral endoscope, its maximum broadband power was compared to that of a clinical endoscope. The maximum power from the clinical endoscope in white light reflectance mode was measured to be $19 \pm 1 \mathrm{~mW}$, at a working distance of $1.0 \pm 0.1 \mathrm{~cm}$. The maximum powers in fluorescence imaging configuration and in reflectance imaging configuration were measured to be $12.9 \pm 0.5 \mathrm{~mW}$ and $10 \pm 1 \mathrm{~mW}$ respectively, at a working distance of $1.0 \pm 0.1 \mathrm{~cm}$. 


\section{B. Resolution and FOV}

The resolution of the device is $240 \pm 20 \mu \mathrm{m}$ as determined using the methods described in Section 3.3.5A. The FOV is governed by the PolyScope accessory channel endoscope, so the FOV of the multispectral endoscope is $63 \pm 1^{\circ}$, as quantified for the bimodal endoscope in Section 4.5.1A.

\section{Spectral response}

The spectral response of the SRDA-based multispectral endoscope determined using the spectrometer and monochromator is shown in Figure 5.7.

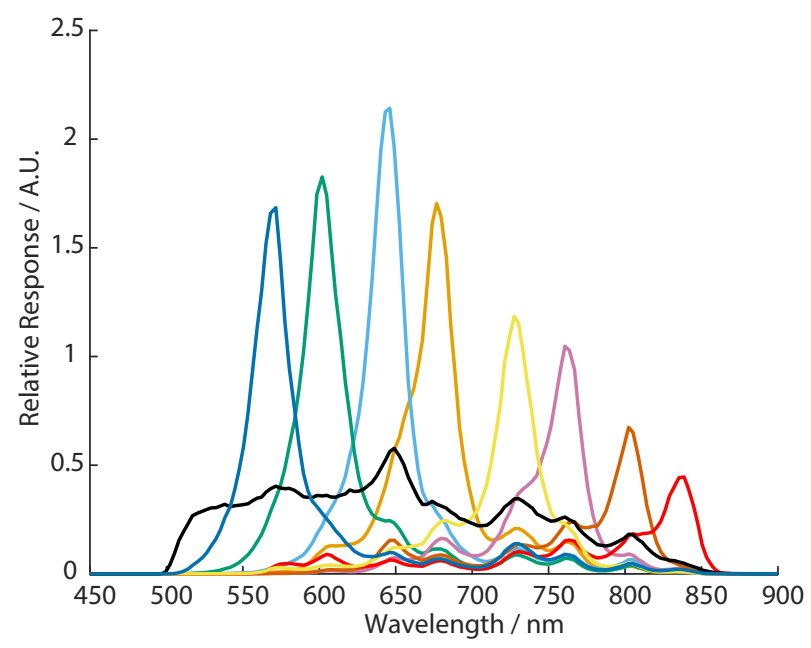

\section{Figure 5.7 The spectral response of the detection arm of the SRDA-based multispectral endoscope.}

\section{Multispectral fluorescence imaging of AF647 in solution}

To validate the performance of the multispectral fluorescence endoscope in MFI, an AF647 dilution series was prepared. Using a spectrometer, the 'ground truth' spectra, $S_{A F 647}(C, \lambda)$, of an AF647 dilution series were recorded, where $C$ denotes the concentration (Figure 5.8 (a)). The reflection spectrum $(\sim 600-650 \mathrm{~nm})$ shows a strong dependence on spatial position of the sample, indicated by the large shaded standard deviation of the 5 measurements. This is expected since reflection is non-isotropic and thus depends on the measurement geometry. The fluorescence emission, $(\sim 650-750 \mathrm{~nm})$, is expected to be isotropic and unaffected by specular reflections from the sample surface, and hence shows a minimal dependence on position. 
The following steps were performed in order to model the reflectance and AF647 fluorescence endmembers.

i. A least squares smoothing spline was fitted to the PBS spectrum, $S_{A F 647}(0, \lambda)$, which should only include reflectance. The resulting spline determined the reflectance signal, $R(\lambda)$ (Figure $5.8(\mathrm{~b})$ ).

ii. This reflectance spectrum, $R(\lambda)$, was fitted (least squares) to each of the AF647 spectra, $S_{A F 647}(C, \lambda)$. The resulting fitted reflectance spectra were subtracted to leave only the 'ground truth' fluorescence spectra $F_{A F 647}(C, \lambda)$ (Figure $5.8(\mathrm{c})$ ).

iii. The peak intensity of each of these fluorescence spectra was used to determine the 'ground truth' abundance of each concentration of dye, $A_{\text {groundtruth }}(C)$ (Figure 5.8 (d)).

iv. For each of the 9 concentrations, the fluorescence spectrum, $F_{A F 647}(C, \lambda)$, was propagated through the spectral response of the multispectral endoscope, $P(B, \lambda)$ (Section 5.5.1C), to yield a fluorescence endmember:

$$
F^{e m}(C, B)=\int F_{A F 647}(C, \lambda) P(B, \lambda) d \lambda
$$

where $B$ is the spectral band of our endoscope $(B=1-9)$.

v. The resulting spectra were normalised and a mean taken across the 9 concentrations to yield a final predicted fluorescence endmember:

$$
F^{e m}(B)=\frac{1}{9} \sum_{C} \overline{F^{e m}(C, B)}
$$

where the bar denotes normalisation to area under the curve $=1$.

vi. Step iv was repeated for the reflectance spectrum, $R(\lambda)$, to yield the final predicted reflectance endmember, $R^{e m}(B)$.

Next, images of the dilution series were captured using the multispectral fluorescence endoscope (Figure 5.8 (e)). A linear weighted sum of the fluorescence and reflectance endmembers was fitted to the image cubes on a pixel-by-pixel basis (Figure 5.8 (f)). The weighting of the fluorescence endmember defines the measured abundance of AF647 for each pixel, $A_{\text {measured }}(C, x, y)$. The mean measured abundance within the region of interest (ROI) within the multispectral image determines the final measured abundance of AF647, $A_{\text {measured }}(C)$ (Figure 5.8 (f)). 


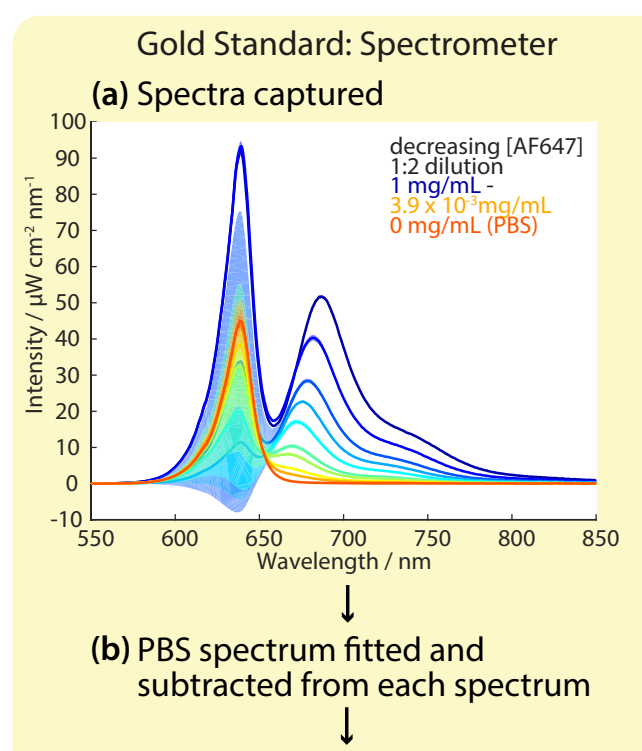

(c) 'Ground truth' fluorescence spectra

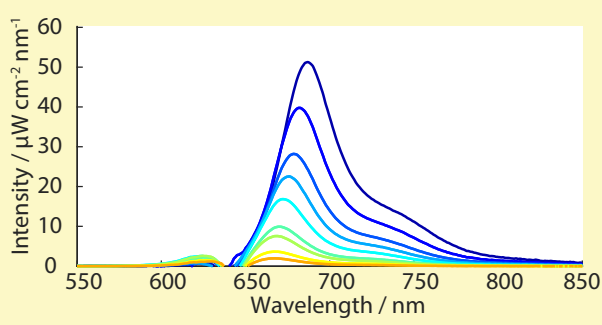

(d) Peak intensity of each spectrum defines 'ground truth' abundance for each concentration
Multispectral Endsocope

(e) Multispectral images captured

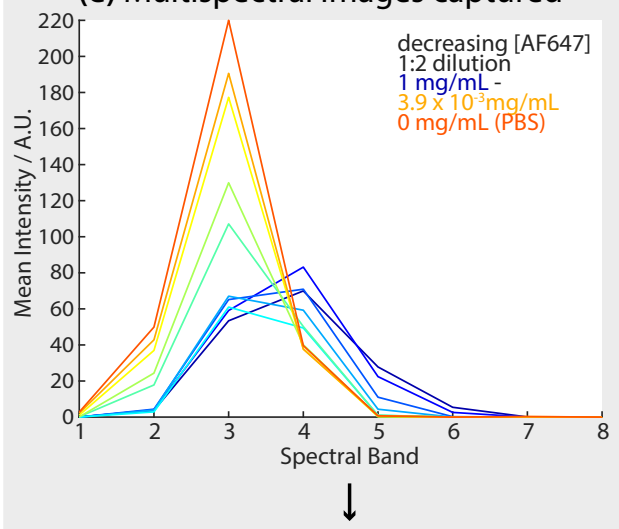

(f) Final endmembers fitted pixelby-pixel to multispectral images (example for a pixel in $1 \mathrm{mg} / \mathrm{mL}$ AF647 ROI)

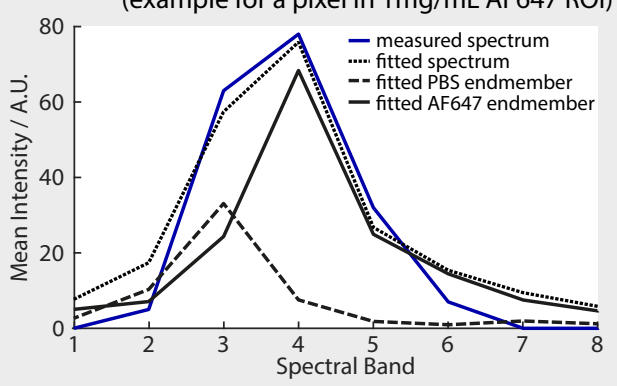

(g) Mean weighting of fluorescence endmember defines abundance for each concentration

\section{Figure 5.8 AF647 abundance measured using the multispectral endoscope and 'ground truth' AF647 abundance measured using a spectrometer.}

A 1:2 dilution series of AF647 was prepared. (a) Spectra of each concentration of AF647 and of PBS were acquired using a spectrometer. The shaded region represents the standard deviation of five spectra acquired with removal and replacement of the sample. (b) The PBS spectrum was fitted to the spectrum for each concentration and subtracted to leave only the fluorescence spectra shown in (c). (d) The peak intensity of each of these spectra defines the 'ground truth' abundance of AF647 for each concentration. (e) Next, the multispectral endoscope was used to capture images of each concentration of AF647 and of PBS. The mean spectra for pixels in the ROI within each of the captured multispectral images are shown. The broad band is omitted for clarity. (f) A weighted sum of the fluorescence and reflectance endmembers was fitted to the image cubes on a pixel-by-pixel basis. An example is shown for a pixel in the $1 \mathrm{mg} / \mathrm{mL}$ AF647. (g) The mean weighting of the fluorescence endmember in the ROI defines the abundance of AF647 for each concentration. The multispectral endoscope has 8 narrow spectral bands; average FWHM $30 \mathrm{~nm}$; centre wavelengths 553, 587, 629, 665, 714, 749, 791, $829 \mathrm{~nm}$. 
A strong correlation $\left(\mathrm{R}^{2}=0.9816\right)$ was found between $A_{\text {measured }}(C)$ and $A_{\text {groundtruth }}(C)$ (Figure 5.9). The small deviation observed at the highest concentration is most likely due to poor off-centre placement of the sample. These results indicate that the multispectral endoscope is capable of accurately unmixing the highly variable background reflectance signal from the fluorescence signal of interest.

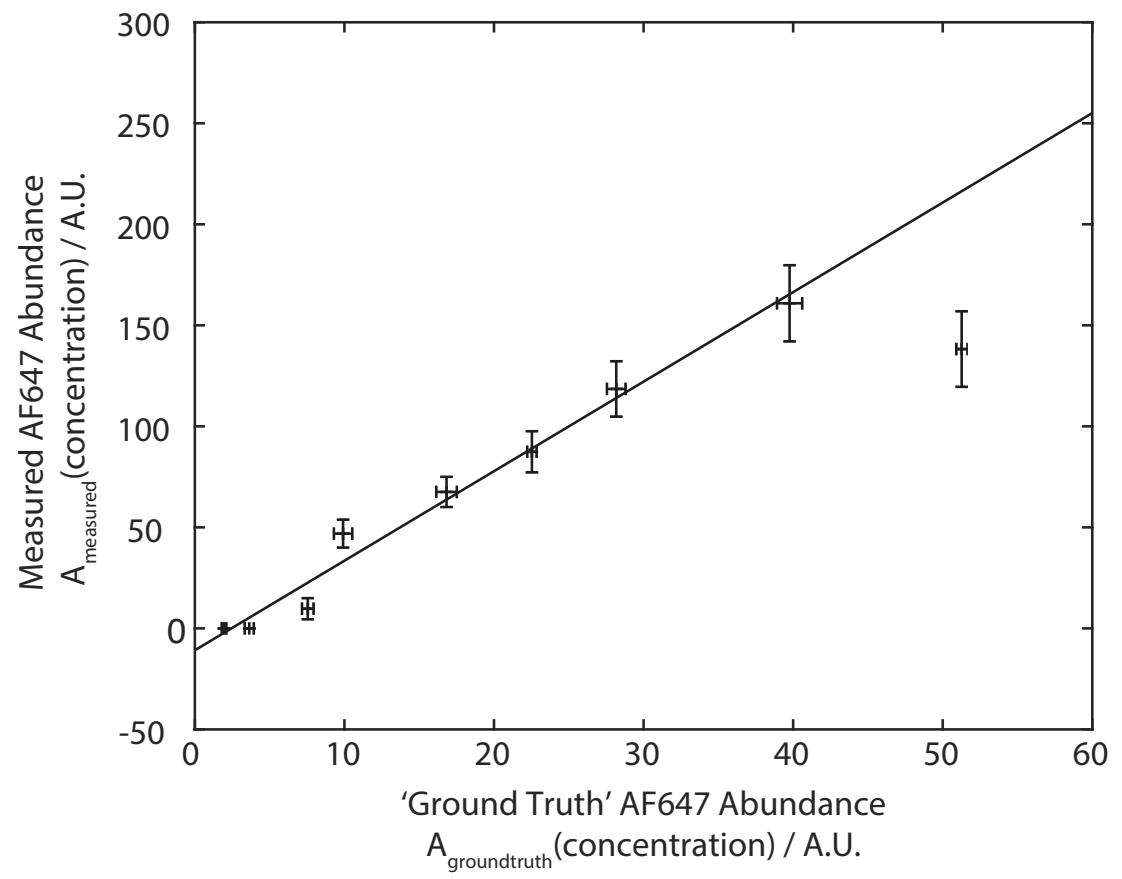

Figure 5.9 Correlation of AF647 abundance measured using the multispectral endoscope with 'ground truth' AF647 abundance measured using a spectrometer.

The measured AF647 abundance is derived by fitting endmembers to the images captured with the SRDAbased multispectral endoscope. The 'ground truth' abundance is determined from the spectrometer measurements. The error in the 'ground truth' abundance is the standard deviation from repeat measurements. The error in the measured AF647 abundance is the standard deviation of abundance over the ROI. A fit is shown for all the data points excluding the highest concentration $\left(\mathrm{R}^{2}=0.9816\right)$.

\section{E. Multispectral fluorescence imaging of multiple fluorophores in ex vivo pig oesophagus}

Having demonstrated the multispectral endoscope is able to accurately image AF647, unmixing two endmembers, one fluorescence and one reflectance, the capability of the endoscope to unmix three endmembers, one reflectance and two fluorescence, AF647 and AF700, was investigated. A whole ex vivo porcine oesophagus was used to simulate clinical endoscopy.

Predicted AF647, AF700 and reflectance endmembers, $E(B)$, were calculated by propagating the data book spectra of AF647, AF700 and the $635 \mathrm{~nm} \mathrm{LED,} S^{D}(\lambda)$, through the spectral response of our multispectral endoscope, $P(B, \lambda)$ (Section 5.5.1C). For example: 


$$
E_{A F 647}(B)=\int S_{A F 647}^{D}(\lambda) P(B, \lambda) d \lambda
$$

where $B$ is the spectral band of our endoscope $(B=1-9)$. These were normalised to area under the curve $=1$.

Each frame of the resulting video was spectrally unmixed by fitting a weighted sum of these endmembers to the 9-point spectrum at each fibrelet centre pixel following demosaicking but prior to interpolation. The weighting of each endmember, AF647, AF700 and reflectance, defines the measured abundance of AF647, AF700 and reflectance respectively. It was demonstrated that the endoscope was capable of detecting both AF647 and AF700 with signalto-background ratios of $310 \pm 90$ and $(2.2 \pm 0.9) \times 10^{4}$ respectively (Figure 5.10).
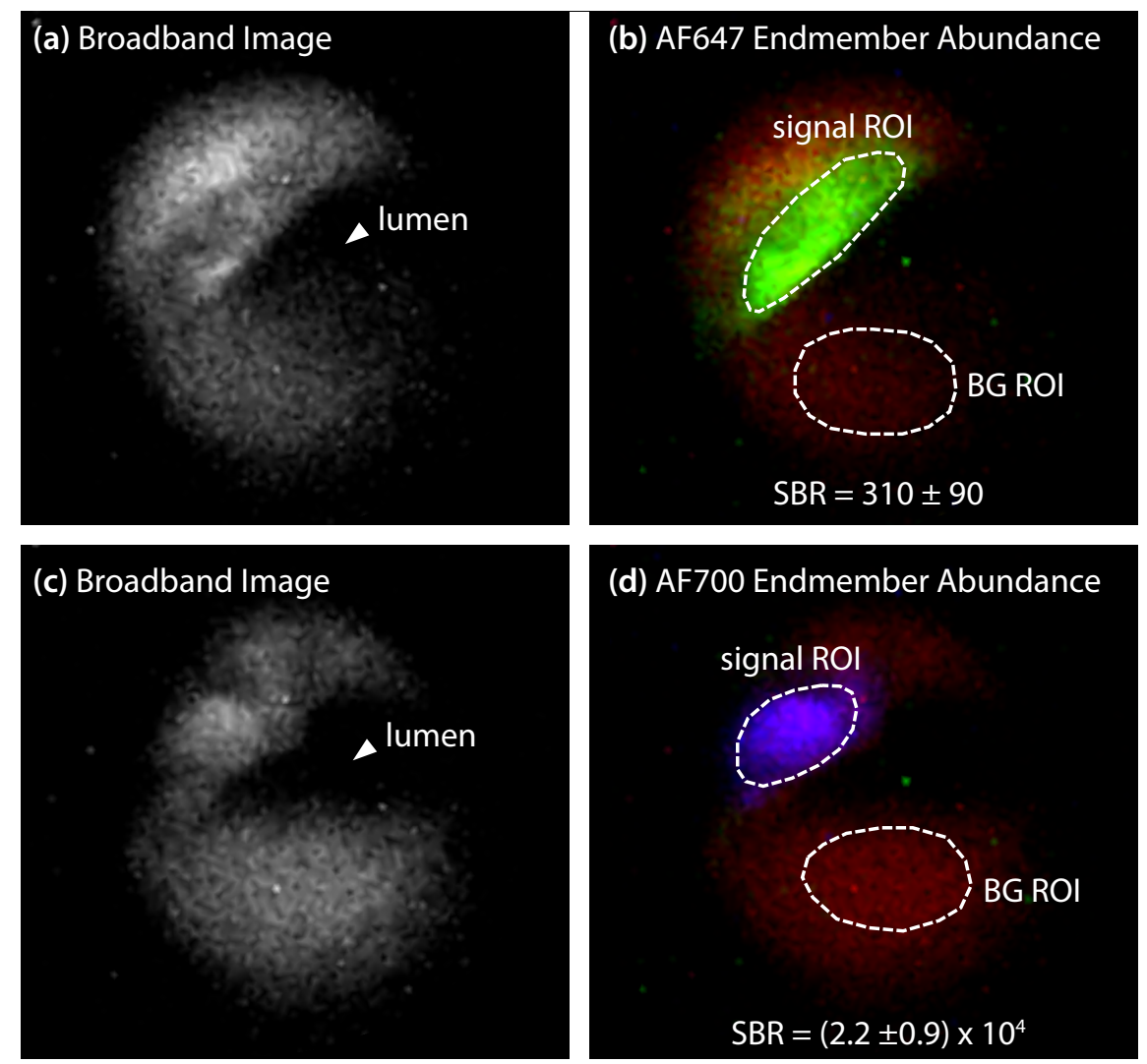

\section{Figure 5.10 Multispectral endoscopic detection of 2 fluorescent dyes (AF647 and AF700) in endoscopy of a whole ex vivo porcine oesophagus.}

(a), (c). Broadband images of oesophageal wall with AF647 and AF700 tissue mimicking phantoms respectively. (b), (d). False colour RGB images (Red: Broadband Image, Green: AF647 endmember abundance from unmixing, Blue: AF700 endmember abundance from unmixing) co-registered to (a) and (c) respectively. Signal and background (BG) regions of interest (ROIs) were drawn and used to calculate signal to background ratio (SBR). 


\subsection{Multispectral Reflectance Imaging of Endogenous Tissue Contrast}

In parallel to MFI, multispectral reflectance imaging of endogenous contrast was investigated. Having developed an SRDA-based multispectral endoscope, written multispectral demosaicking and decombing algorithms, and proven the feasibility of imaging with the device in an ex vivo simulation of endoscopy, and since ex vivo tissue does not represent a good biological model of endogenous tissue contrast (Translational Barrier 4), work using multispectral reflectance endoscopy proceeded directly to in vivo trials.

Thus an in vivo single centre pilot cohort study was designed, entitled: "Prospective pilot cohort study to assess feasibility of multispectral endoscopic imaging for detection of early neoplasia in Barrett's oesophagus", or "Multispectral endoscopy (MuSE)" for short. For this trial, 20 patients will be recruited, the patient population enriched such that approximately $40-50 \%$ will have dysplasia at the study endoscopy. Imaging with the multispectral endoscope will occur as part of the subjects' clinically indicated procedure.

\subsubsection{Methods}

\section{A. Trial objectives}

The trial is designed to address two primary objectives:

i. To assess the feasibility of imaging with the novel multispectral endoscope, ensuring the device can be used to capture images in a clinical environment and with minimal interruption to clinical workflow.

ii. Evaluate the appearance of Barrett's oesophagus with and without dysplasia using multispectral endoscopy to build a library of potentially diagnostically useful distinguishing spectral characteristics since these are currently unknown and not easily investigated using ex vivo tissue (Translational Barrier 4).

A long-term secondary objective is also proposed, which can be addressed once a large data set of multispectral images of confirmed pathologies has been acquired and a set of imaging biomarkers to distinguish Barrett's oesophagus with and without dysplasia has been established:

iii. Evaluate diagnostic accuracy of multispectral endoscopic image derived biomarkers for Barrett's oesophagus with dysplasia. 


\section{B. Trial design}

Each procedure is performed by a single endoscopist.

i. After local anaesthesia or conscious sedation (Midazolam $+/$ - Fentalyl) the endoscopist intubates the patient with a high resolution white light therapeutic endoscope (GIFH290Z, Olympus, Japan). The use of the therapeutic scope is required given the larger accessory channel (3.7 $\mathrm{mm}$ diameter), which is compatible with the insertion of the PolyScope catheter $(3.0 \mathrm{~mm}$ diameter). The endoscopist thoroughly cleans the oesophageal mucosa using standard cleansing agents. The endoscopist thoroughly inspects the mucosal surface of the oesophagus. Cautery marks are placed around suspicious lesion(s) and around one region of Barrett's oesophagus as a control (Figure $5.11(\mathrm{a}))$.

ii. The endoscopist inserts the multispectral endoscope through the working channel and uses this to inspect the Barrett's oesophagus segment with particular attention to the regions outlined in step i (Figure 5.11 (b)).

iii. The endoscopist switches back to conventional imaging. The endoscopist takes endoscopic mucosal resections from each of the suspicious lesion(s). Random biopsies are taken according to the Seattle protocol. The random biopsies are omitted if they are considered unnecessary for more advanced disease (e.g. clearly visible intramucosal cancer which needs endoscopic resection).

Pathological assessment of biopsies is performed according to the revised Vienna classification [215]. The HD-WLE video stream is recorded using a recording unit (SMP300, Extron, USA).

(a)

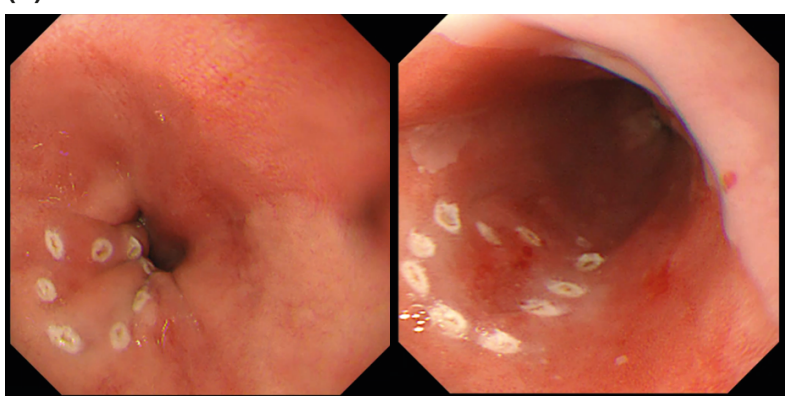

Barrett's Oesophagus

Suspicious Lesion (b)

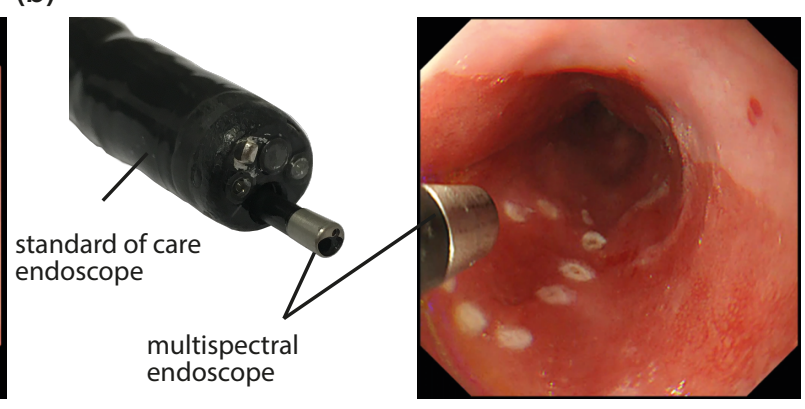

Figure 5.11 Procedure for in vivo clinical trial of multispectral endoscopy (MuSE).

(a) Suspicious lesion(s) are identified and marked using standard of care HD-WLE. (b) The multispectral endoscope is inserted via the working channel of the standard of care endoscope. 


\subsubsection{Results (MuSE 01)}

The trial was approved on $2^{\text {nd }}$ March 2018 (Appendix A). The first patient was recruited and the first trial performed on $30^{\text {th }}$ May 2018 (MuSE 01). Approximately one hour prior to the procedure, the PolyScope 10,000 fibre imaging bundle was threaded into a sterile $185 \mathrm{~cm}$ PolyScope catheter (PD-PS-0144p, PolyDiagnost, Germany), which was handled with sterile gloves to ensure the region intended to be in direct patient contact remained sterile (Figure 5.2). The system was aligned, the catheter illumination fibre was connected to the light source via the custom coupler, and the power was checked to be $10 \pm 1 \mathrm{~mW}$ using the method described in Section 4.5.1B. Then, the standard of care endoscopy began with the standard of care endoscope (Lucera GIF-2T240, Olympus, Japan).

Following inspection of the whole Barrett's segment with HD-WLE, one suspicious lesion was identified. Cautery marks were placed around this suspicious lesion and around one region of Barrett's oesophagus as a control (Figure 5.11 (a)). Next, the multispectral endoscope was threaded into the working channel of the standard of care endoscope. Data was captured for 12 minutes at $\sim 1$ fps, resulting in 842 SRDA images and 842 gold standard spectra. Finally, the multispectral endoscope was withdrawn to allow standard of care endoscopy to continue.

At this time of this procedure, the suspicious lesion was not resected due to risk of excessive bleeding from the patient. On $8^{\text {th }}$ June 2018, the patient returned and the suspicious lesion was resected using endoscopic mucosal resection (EMR). The EMR specimen was sent to histopathology for diagnosis. A pathology grid was generated as described in Section 4.6.1C. Histopathology confirmed the presence of moderately differentiated intramucosal adenocarcinoma (Figure 5.12).

(a)

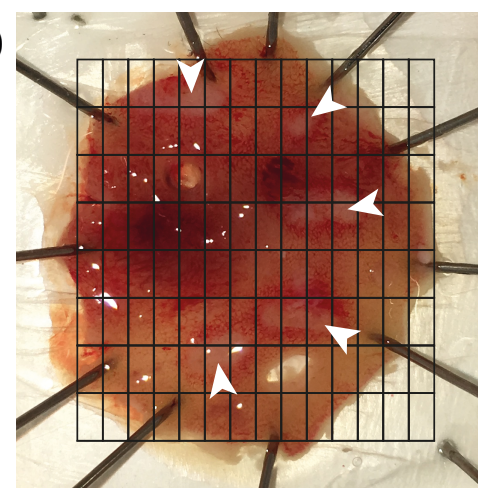

(b)

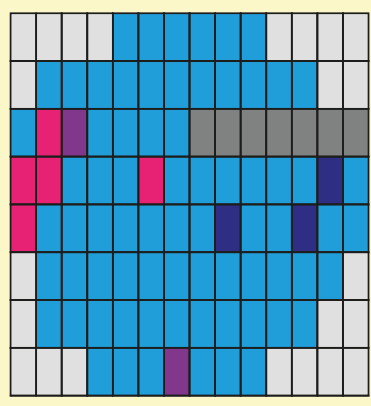

Excluded

Artefact

Normal squamous

Non-dysplastic Barrett's

(with intestinal metaplasia)

Low grade dysplasia

High grade dysplasia

Intramucosal carcinoma

\section{Figure 5.12 EMR specimen from MuSE 01.}

(a) White light image of the EMR specimen from MuSE 01. The location of two punch biopsies are visible in row 3 column 5 and row 7 column 9. Cautery marks are also visible (white arrows). (b) Histopathology grid manually coregistered to the white light image. This confirms the presence of moderately differentiated intramucosal carcinoma in the sample. 
Analysis of the multispectral endoscopy data was carried out in Matlab® (MathWorks, USA). The raw images captured by the SRDA were demosaicked and decombed using the interpolation methods outlined in Section 3.3.3. A false colour multispectral endoscopy video was generated by assigning the narrow bands centred at 629,587 and $553 \mathrm{~nm}$ to RGB channels respectively.

This multispectral endoscopy video and the HD-WLE video were synchronised and placed side by side for viewing (Figure 5.13). In much of the video, the endoscopist is articulating the standard of care endoscope to bring the marked regions into the field of view of the multispectral endoscope. The video was used to identify times where this was achieved. A total of 17 images and 25 gold standard spectra, from 2 short video segments viewing the Barrett's tissue and 3 short video segments viewing the cancer tissue, were selected.

In the false colour multispectral images, the cautery marks surrounding the regions of interest appear as bright white patches. These were used to guide the drawing of regions of interest (ROIs) in the images (Figure 5.13 (b)). Inside each ROI, the mean across each spectral band image was calculated to yield a 9-band image spectrum. These were normalised to AUC $=1$. The mean of the 9-band image spectra for Barrett's ROIs $(n=9)$ and cancer ROIs $(n=8)$ are plotted in Figure 5.14 (a). These spectra show encouraging differences between the two disease types, particularly in bands 1-4 (average FWHM $30 \mathrm{~nm}$; centre wavelengths 553, 587, 629, 665 $\mathrm{nm})$.

The gold standard spectra were background subtracted and normalised to AUC $=1$. The mean of these spectra for Barrett's $(n=9)$ and cancer $(n=16)$ are plotted in Figure 5.14 (b). These spectra show encouraging differences between the two disease types in more detail in the region from 520-650 $\mathrm{nm}$. To allow comparison to the 9-band multispectral image spectra, these background subtracted, gold standard spectra, $S^{g}(B)$, were propagated through the spectral response of our endoscope, $P(B, \lambda)$ (Section 5.5.1C) to yield modelled 9-band image spectra:

$$
S^{g}(B)=\int S^{g}(\lambda) P(B, \lambda) d \lambda
$$

These were normalised to AUC $=1$. The mean over the modelled 9-band image spectra for Barrett's and cancer are plotted in Figure 5.14 (c). These compare favourably to the measured 9-band image spectra from the SRDA (Figure 5.14 (a)). The small standard deviation of both image spectra and gold standard spectra, shown shaded in Figure 5.14, suggest high repeatability of the technique. 


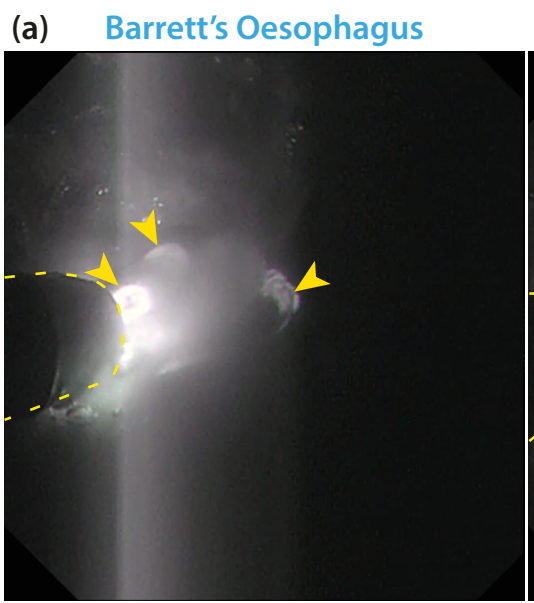

Intramucosal Adenocarcinoma

Intramucosal Adenocarcinoma
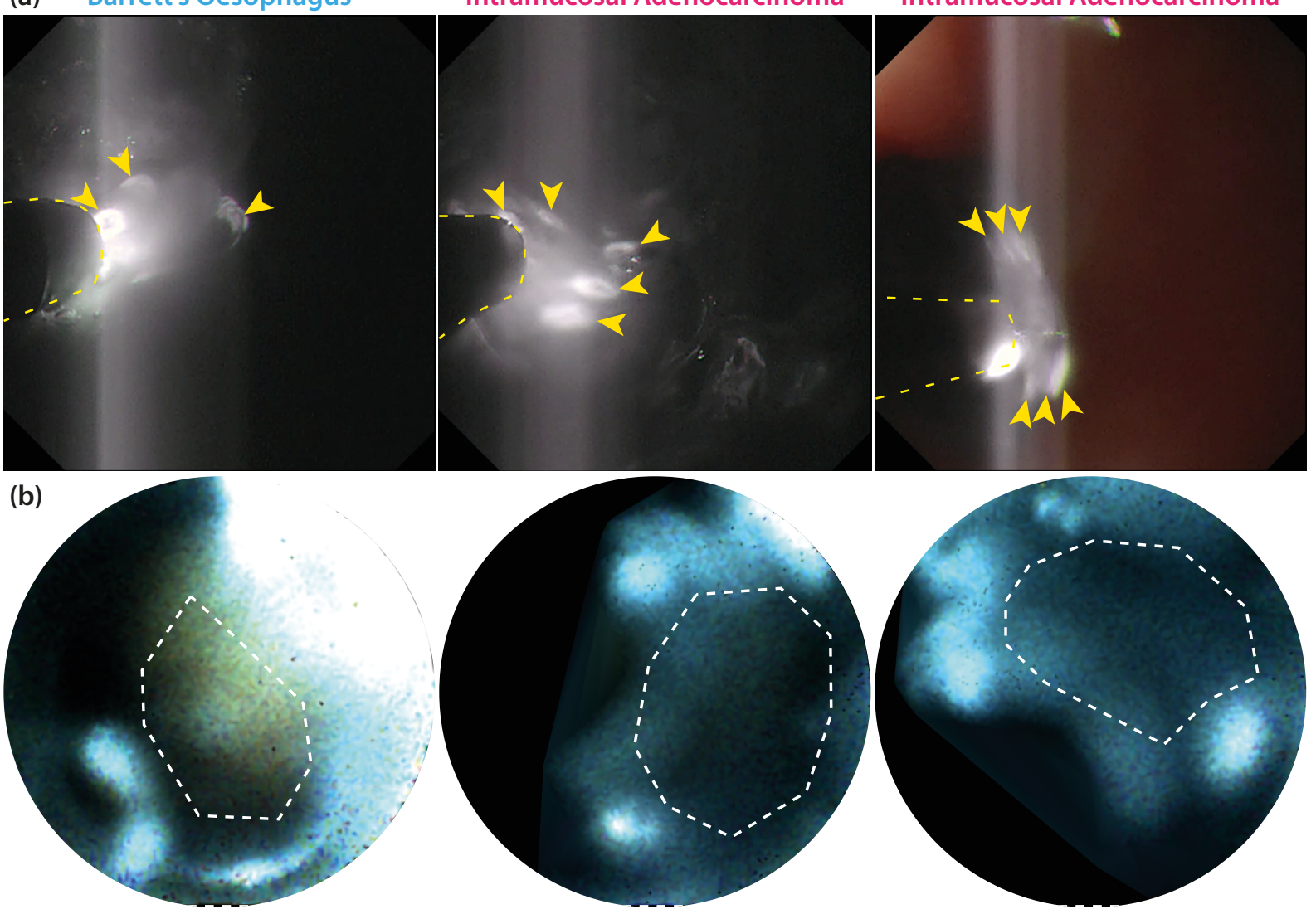

Figure 5.13 Synchronised HD-WLE and multispectral images from the first-inhuman trial of the multispectral endoscope (MuSE 01).

(a) High definition white light endoscopy (HD-WLE) images from MuSE 01. Images are from three of the selected video segments. From left to right: Barrett's oesophagus, intramucosal carcinoma, intramucosal carcinoma. The cautery marks delineating the regions can be seen as bright white patches in these images (yellow arrows). The yellow dashed lines indicate the position of the multispectral endoscope to aid visualisation. The images are dark as the HD-WLE light source is switched off for multispectral imaging. (b) Multispectral images corresponding to the three HD-WLE images. These false colour RGB images are generated by assigning the narrow bands centred at 629, 587 and $553 \mathrm{~nm}$ to RGB channels respectively. The cautery marks are visible as bright white patches. Regions of interest (ROIs) are shown (white dashed lines). 


\section{_ Barrett's Oesophagus} Intramucosal Carcinoma

\section{* propogated through spectral} repsonse of endscope
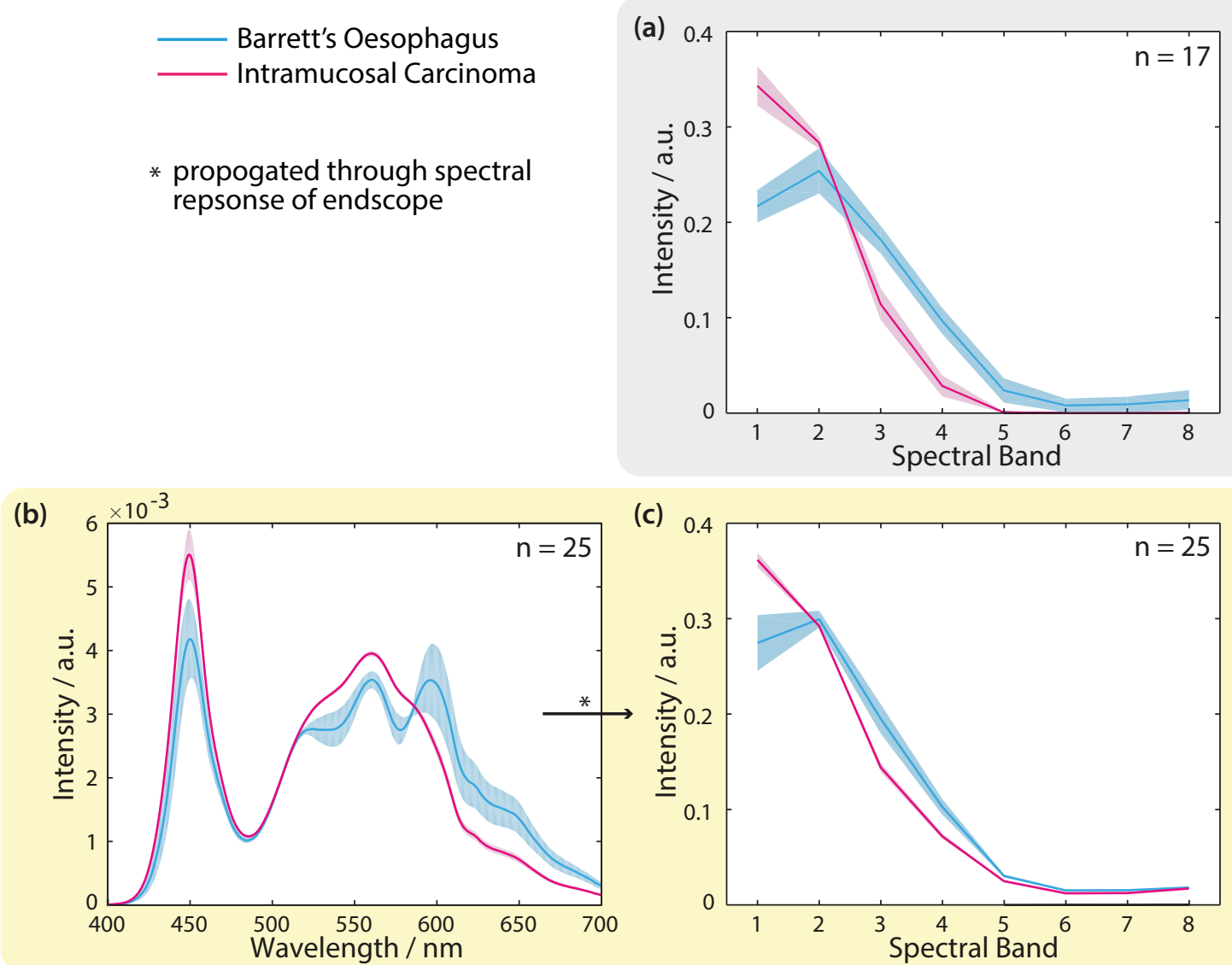

\section{Figure 5.14 Spectra of Barrett's oesophagus and intramucosal carcinoma measured with the multispectral endoscope.}

(a) Mean multispectral image spectra from within the ROIs. Shaded areas represent the standard deviation across the ROIs ( $\mathrm{n}=17$ images; 9 Barrett's oesophagus, 8 intramucosal carcinoma). The 8 narrow spectral bands are shown; average FWHM $30 \mathrm{~nm}$; centre wavelengths 553, 587, 629, 665, 714, 749, 791, $829 \mathrm{~nm}$. (b) Mean spectra captured by gold standard spectrometer. (c) The gold standard spectra were propagated through the spectral response of the multispectral endoscope (Section 5.5.1C) to yield modelled 9-band image spectra for comparison with the multispectral image spectra. Shaded areas represent the standard deviation across the spectra ( $\mathrm{n}=25$ spectra; 9 Barrett's oesophagus, 16 intramucosal carcinoma). 


\subsection{Discussion and Conclusions}

To address the clinical challenge of Barrett's surveillance, an SRDA-based multispectral endoscope capable of acquisition of 9-band multispectral images in vivo was developed. Two alternative optical configurations of the device were tested. The first, designed for multispectral fluorescence imaging, was demonstrated to be capable of accurately detecting fluorescent contrast agents both in well plates and in a realistic clinical scenario simulated using a whole ex vivo porcine oesophagus. The second, designed for multispectral reflectance imaging of endogenous contrast, has been applied in a first-in-human clinical trial, where the measured spectra showed promising differences between Barrett's oesophagus and cancer tissue.

While these results are promising, some challenges remain for further clinical translation of SRDA-based multispectral imaging. The most significant challenge facing SRDA-based multispectral imaging is the limited sensitivity of the device due to the low sensitivity of the SRDA, partly due to the low quantum efficiency (QE) of the underlying sensor $(<60 \%)$, and partly due to the low transmission of the CFA ( $40 \%)$. Increased sensitivity is important in order to increase signal for both multispectral reflectance and multispectral fluorescence imaging, but it is particularly important for the latter, where low concentrations of dye must be detected. Furthermore, increased sensitivity enables shorter exposure times, thus allowing frame rate to be increased to achieve video rate imaging ( 25 frames per second).

This challenge could be overcome in a number of ways, with increasing complexity. Firstly, and most simply, the illumination power of the system could be increased. This should increase reflectance signal, and fluorescence signal so long as saturation of the fluorophores is avoided. Secondly, should an appropriate collaboration be initiated, the CFA could theoretically be deposited on a higher QE sensor, though this would be expensive. Finally, as a more long-term solution, attenuation associated with imaging though a fibre bundle could be avoided by miniaturising SRDAs and placing them on the tip of the endoscope. Since this requires significant research and development resources, it would likely be executed by one of the major endoscope vendors, and require considerable evidence of the clinical utility of the technique prior to investment.

The large data processing requirements also present a challenge. Currently we perform our spectral unmixing offline, with this taking around 0.025 seconds per pixel (MacBook Pro, 2.4 GHz Intel Core i5, Memory 8 GB $1600 \mathrm{MHz}$ DDR3), to unmix 4 endmembers, corresponding to around 9 hours per $1024 \times 1280$ pixel image. Fortunately, since imaging occurs through a fibre bundle, only pixels at the 10,000 fibrelet centres need to be unmixed prior to interpolation, 
reducing the processing time to 4 minutes per image. For real time classification, this could be reduced by utilising GPUs, or reducing the number of spectral bands used for unmixing.

Future work should investigate more advanced spectral unmixing techniques. These have been developed for application in a wide range of fields including earth observation, food safety monitoring, pharmaceutical process monitoring, forensics and recently biomedical imaging $[170,245]$. Spectral unmixing can be either supervised, where a priori knowledge of endmembers has been used to train the algorithm, or unsupervised, where the algorithm must determine both the endmembers and their abundances based on the variation within the data.

For biomedical spectral unmixing, the endmembers associated with different disease states or tissue types might initially be unknown, hence the need for exploratory pilot studies similar to the MuSE trial presented here. These studies are important, not only because they allow determination of the endmembers in vivo, but because they allow us to determine the intrapatient and inter-patient heterogeneity of these endmembers.

Should the discovered endmembers be well conserved, they can then be used as a priori knowledge for unmixing algorithms. In the simplest case, this means reducing the unmixing problem to an inversion problem aimed at determining the relative abundance of the known endmembers from a measured dataset (e.g. least squares unmixing), but these methods are sensitive to heterogeneity. A more advanced approach is to use a gold-standard dataset, perhaps that collected during the exploratory pilot study, to train a supervised unmixing algorithm (e.g. random forest, neural network). For proper training, a large, well-annotated dataset is required, meaning the images must be clearly outlined and labelled, a time consuming process. However, the larger this training dataset, the more robust the algorithm to inter-patient and intra-patient heterogeneity.

If the endmembers are not conserved between patients, unsupervised unmixing can be used to determine new endmembers in each patient (e.g. k-means clustering, principle component analysis, autoencoders), but this does not supply labels for the segmentation; these must be input by the user.

Ideally, the colour filter array of the SRDA should be optimised for the detection of the differences between spectral signatures of each disease type, whether these be fluorescence or reflectance, potentially increasing sensitivity whilst limiting the number of spectral bands needed to unmix the spectra of interest, thus decreasing processing time. However, producing a one-off custom CFA is prohibitively expensive. In the long term, once the spectral signatures of Barrett's and dysplasia are well validated in single centre clinical trials, and their clinical 
utility is established, manufacture of multiple custom CFAs for large scale multicentre trials might be more economical, and more likely to gain the significant investment required.

Despite these outstanding challenges, the SRDA-based multispectral endoscope presented here has many advantages: it has received local safety approval for use in humans; it is compatible with insertion through the working channel of standard of care endoscopes, which enabled easy implementation during the first-in-human trials (Translational Characteristic 2); it can be articulated using the familiar standard of care endoscope, which allowed it to be positioned in order to image particular regions of interest in the oesophagus (Translational Characteristic 3). Furthermore, MSI occurs directly on the SRDA, meaning the compact device has fewer optical components, and is more resistant to misalignment than alternative multispectral imaging techniques (Translational Characteristic 2). Additionally, the multispectral data can be used to produce a false colour RGB image to guide the endoscopist (Translational Characteristic 6).

Several steps must be taken to continue the translation of the two multispectral imaging approaches investigated here. For MFI, much of the technical validation in Domain 2 of the OIB Roadmap has been completed (Figure 1.3): the device has been shown to accurately image fluorophores using a dilution series; the ability to detect fluorophores in a more realistic setting has been demonstrated using ex vivo pig oesophagus; and the device is available, compatible with the clinical environment and approved for use in humans.

Biological validation of the device requires experiments in which the multispectral endoscope is used to image a targeted molecular imaging probe applied to ex vivo human tissue, similar to those described in Chapter 4.6. These experiments biologically validated WGA-IR800 paired with the bimodal endoscope for delineating dysplasia from Barrett's oesophagus. The underlying principles should apply to MFI paired with WGA conjugated to any fluorophore. However, to ensure thorough biological validation, the experiments should be repeated using the multispectral fluorescence endoscope paired with a particular WGA conjugate. Since it is not yet clear when (or which) targeted molecular imaging probes will be manufactured under GMP conditions, and ultimately approved for use in humans, these experiments were deferred to a later date and attention turned to imaging endogenous contrast.

For multispectral reflectance imaging of endogenous tissue contrast, much of the technical and biological validation required in Domain 2 of the OIB Roadmap (Figure 1.3) is not easily performed using ex vivo tissue. When tissue is excised from the body, endogenous tissue features change irreversibly (Translational Barrier 4). For example, the lack of active blood flow changes the spectrum of tissue by reducing blood oxygenation to $0 \%$ and consequently altering the haemoglobin absorption spectrum. Furthermore, tissue autofluorescence can be modified upon exposure to ambient light and tissue structure may be distorted by surgical 
trauma, or by positioning the tissue on a rigid surface. Ultimately, tissue will degrade unless fixed in formaldehyde or frozen, which further alters properties [55]. The leap between data acquired during ex vivo and in vivo imaging is thus large and data acquired from ex vivo tissues may contain insurmountable artefacts if the tissues are not properly handled, justifying our decision to proceed directly to in vivo multispectral reflectance imaging in a clinical pilot study (MuSE).

In the first trial of this pilot study, MuSE 01, multispectral endoscopy produced promising results. Distinct reflection spectra were observed for regions of Barrett's oesophagus and cancer, suggesting the potential to delineate diseased tissue based on endogenous reflectance using the multispectral endoscope. These spectra were consistent over multiple viewpoints separated by several minutes of imaging, suggesting high repeatability of the approach.

This work also has several limitations. The majority of the spectral differences were observed in the region from $530-650 \mathrm{~nm}$, but since the light source had little power above $700 \mathrm{~nm}$, the reflectance spectrum above $700 \mathrm{~nm}$ was not explored. Since our endoscope can image light up to $850 \mathrm{~nm}$ (Figure 5.7), a more broadband light source will be installed for future trials.

The ultimate aim is to delineate dysplasia from Barrett's oesophagus. The majority of the suspicious lesion imaged in MuSE 01 was composed of intestinal metaplasia, with some highgrade dysplasia, but intramucosal cancer was also present. Future trials with exclusively dysplastic lesions are required to evaluate the appearance of dysplasia on MSI, and thus test the potential of the technique to perform our ultimate objective of delineating dysplasia from Barrett's oesophagus.

In MuSE 01, the coregistration challenges described in Chapter 4 were avoided by delineating regions of interest with cautery marks and taking biopsies of the marked regions following multispectral imaging. However, positioning the multispectral endoscope to image the marked regions is difficult, and further trials will be required to practice and improve this. In addition, biopsies from healthy tissue regions were not taken, meaning gold standard diagnosis of these regions relies on the less reliable classification from the endoscopist inspecting the tissue using HD-WLE. In future, biopsies should be taken from the healthy region to confirm the tissue classification with gold standard histopathology.

Nevertheless, considering this was the first trial of the multispectral endoscope in vivo, MuSE 01 went some way to addressing both the primary objectives of our trial (Section 5.6.1A): it was confirmed that the device can be used to capture images in a clinical environment and with minimal interruption to clinical workflow; the appearance of Barrett's oesophagus without dysplasia (but not yet with exclusively dysplasia) using multispectral endoscopy was evaluated, 
the first step to building a library of diagnostically useful distinguishing characteristics. Given this initial success, a second patient is due to be recruited in late September 2018 (MuSE 02). Over the next 12 months, we aim to recruit 10-20 patients in MuSE, while iteratively making improvements to the device, optimising it to image the emerging OIBs, and to the methodology, optimising coregistration of the in vivo images with histopathology. With this trial underway, and having demonstrated the potential of multispectral imaging for delineation of disease, we proceeded to apply SRDA-based multispectral imaging in rigid endoscopy for the delineation of brain cancer. The development of this system is discussed in the following chapter. 


\section{RIGID ENDOSCOPY FOR INTRAOPERATIVE IMAGING OF PITUITARY ADENOMA}

In the previous chapters, optical imaging was discussed in the context of upper endoscopy for the surveillance of Barrett's oesophagus, where a wide range of light-tissue interactions (Figure 1.1) have been utilised and investigated thoroughly in many novel optical imaging techniques, some of which have been deployed in vivo. In contrast, for intraoperative pituitary imaging the majority of light-tissue interactions are yet to be investigated, leaving many potential contrast mechanisms unexploited.

\subsection{Pituitary Adenoma}

The pituitary gland is a pea-sized organ situated behind the ridge of the nose, attached to the base of the brain by a thin stalk. It is a key component of the endocrine system, responsible for hormonal control of other glands as well as many aspects of normal functioning including growth and blood pressure. Pituitary adenoma, the development of a benign tumour, is the most common disease associated with the pituitary gland, with a prevalence of approximately $17 \%$ in the US population, although the majority cause no symptoms [246]. These are a diverse group of tumours, which may be functional or non-functional [246]: functional adenomas usually present with clinical symptoms specific to the increased hormone secretion, while nonfunctional adenomas present due to mass effect resulting in visual loss due to compression of the optic chiasm or identification incidentally on imaging for other indications [247]. 


\subsubsection{Standard of Care}

Transsphenoidal surgical resection is the primary treatment method for pituitary adenomas (Figure 6.1) [248]. A surgeon inserts rigid endoscopic devices through the nostrils and to the back of the nasal cavity where small openings are made in three bones, the nasal septum, sphenoid sinus and the sella, to reach the pituitary gland. The surgeon then removes the adenoma guided by white light imaging followed by closing of the sella. It is often difficult to determine the difference between normal pituitary gland, which must be preserved to minimise loss of function, and the pituitary tumour.

Following transphenoidal surgery, cure rate for acromegaly related adenomas is reported between 46-79\% depending on surgical experience [249]. Remission for Cushing's disease related adenomas following transphenoidal surgery is reported at $89 \%$ [250]. Reoperation is difficult: scar formation may distort anatomy and impair adequate resection of tumours. This increases the risk of complications such as loss of normal pituitary function and carotid artery rupture [251]. Furthermore, recurrent tumours are often more rigid [252], and landmarks may be distorted from preoperative radiotherapy or other treatments [253]. Hence, a delicate balance must be struck between maximising completeness of resection and preserving endocrine function from the normal pituitary, so delineation of normal pituitary from adenoma is paramount [254]. Hence there is an unmet clinical need for novel imaging techniques capable of assisting the surgeon in delineating normal pituitary tissue from adenoma.

\subsubsection{Advanced Imaging of Pituitary Adenoma}

Currently, surgeons rely on pre-operative MRI in order to try and determine where the normal pituitary gland might be in relation to the tumour, for example, if the pituitary stalk is displaced to the right, it is likely that the normal pituitary gland will also be displaced to the right. Following resection, post-operative MRI is also used as the gold standard for assessing the extent of resection. However, the images may contain artefacts from hematoma or surgical packing, so postoperative MRI often takes place a few months after the surgery, when these artefacts are no longer present [255], too late to inform changes to the initial surgery.

MRI can also occur during surgery. Intra-operative MRI (iMRI) offers immediate feedback to surgeons following resection, allowing them to re-examine the site of any residual tumour that shows up on the MRI images. However, it is expensive, potentially requiring extensive remodelling of operating theatres to accommodate the MRI scanner and it prolongs surgery [255]. Furthermore, image artefacts can lead to false positives, so iMRI is not recommended by the Congress of Neurological Surgeons [248]. In recent years, there has been much research into intraoperative optical imaging technologies for transsphenoidal surgery, including narrow 


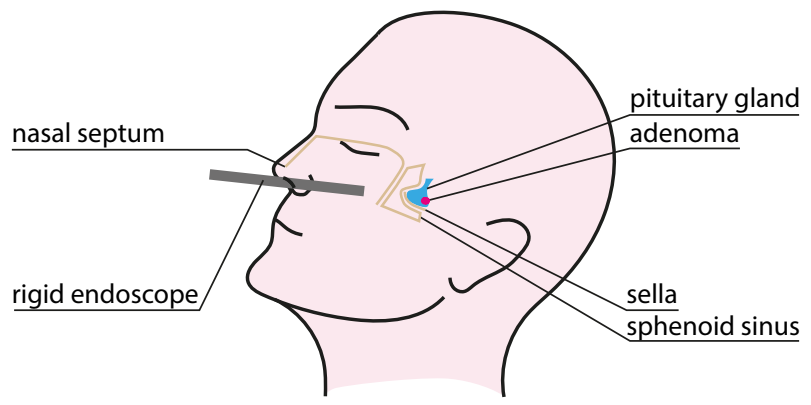

Figure 6.1 Simple schematic showing transphenoidal surgery for pituitary adenoma.

band imaging (NBI) [256], 4K imaging [257], ICG fluorescence imaging [258, 259] and optical molecular imaging (OMI) with a fluorescent folate analogue [260].

\section{A. $4 K N B I$}

Narrow band imaging (NBI) was described in detail in Section 2.2.2B. On standard NBI, the pituitary gland appears as an arabesque pattern due to the rich vasculature, whereas the tumour has no particular pattern of enhancement [256]. A recently released $4 \mathrm{~K}$ ultra-high definition (UHD) NBI endoscope (VISERA, Olympus, Japan) allows $3840 \times 2160$ pixel UHD imaging with NBI, allowing high resolution imaging of the microvasculature [257]. with a short learning curve, since it is similar to familiar standard NBI (Translational Characteristic 3, Translational Characteristic 4). However, the system is bulky (Translational Characteristic 2), using a 55-inch monitor, and the colour balance must be delicately tuned to allow sustained visualisation during bleeding. It remains to be seen whether UHD NBI will make any lasting impact to the SOC.

\section{B. ICG fluorescence imaging}

Indocyanine green (ICG) is an intravenously administered green fluorescent dye which binds to blood plasma albumin allowing it to be used for vascular imaging. It has a short half-life, an acceptable safety profile and fluoresces in the NIR, allowing good depth penetration (up to a few $\mathrm{mm}$ ). There is lower vasculature density in adenomas compared to the healthy anterior pituitary [261, 262], so several groups have performed ICG fluorescence endoscopy in transphenoidal surgery for benign pituitary lesions [258, 259]. ICG is injected and allowed to circulate before imaging, this timing being crucial; the internal carotid artery reaches peak fluorescence first, followed by the intercavernous sinus and then the pituitary itself [263].

In work by Litvack et al. there was higher fluorescence in the pituitary gland than in the tumour ( $\mathrm{n}=12$ patients), but this pilot study was limited by their standard definition endoscope and a lack of quantitative objective results [259]. Their custom endoscope was developed by Karl 
Storz and a second-generation device is in development. Sandow et al. found that adenoma could be detected, either by lower ICG intensity compared to surrounding tissue, or higher intensity due to uptake [258] with the type of contrast associated with the clinical symptoms of the patient ( $\mathrm{n}=22$ patients) [258]. This variation of ICG signal characteristics makes ICG based resection difficult due to lack of specificity [258]. In contrast to flexible NIR fluorescence endoscopes (Chapter 4), rigid NIR fluorescence endoscopes are commercially available, these being intended for imaging of ICG, but further development is needed since these are currently $\geq 5 \mathrm{~mm}$ in diameter compared to $4 \mathrm{~mm}$ standard endoscopes, and neither side facing nor tipwashing devices are available (Translational Characteristic 2) [263, 264].

\section{Optical molecular imaging}

Intraoperative optical molecular imaging of folate receptors has been proven feasible and beneficial in ovarian cancer ( $\mathrm{n}=10$ patients [265], $\mathrm{n}=12$ patients [266]). Folate receptor alpha (FR $\alpha)$ overexpression has also been reported in non-functioning pituitary adenomas [267-269]. Using OTL38, a fluorescent folate analogue, and a commercially available NIR fluorescence endoscope (Iridium, Visionsense, USA), Lee et al. improved true positive rate and true negative rate for detecting pituitary adenoma in margin specimens from $80 \%$ and $89 \%$ using white light alone, to $86 \%$ and $89 \%$ respectively, but many adenomas did not overexpress FR $\alpha$ (no functioning adenomas and half of the non-functioning adenomas overexpressed FR $\alpha$ ), a significant limitation [260]. Their future work will focus only on non-functional adenomas since none of the functioning adenomas overexpressed FR $\alpha$ [260].

\section{Multispectral imaging}

ICG fluorescence and optical molecular imaging face translational challenges associated with the use of exogenous contrast (Translational Characteristic 1). As mentioned previously, multispectral imaging has the potential to use spatially resolved spectral data to delineate disease based on endogenous contrast [10, 223]. Furthermore, additional spectral information may be used to correct for working distance [145, 217], which was a critical confounder for optical molecular imaging with OTL38 in the work by Lee et al. [260].

MSI has not yet been used in pituitary imaging but liquid crystal tuneable filter (LCTF) based MSI has been used to capture reflectance and fluorescence image cubes in human glioblastoma, an aggressive brain cancer [270]. Using the reflectance signal at 465-485 nm and 625-645 nm, the fluorescence signal was corrected to yield quantitative fluorescence images displaying intrinsic fluorescence independent of optical properties of the tissue [270]. 
SRDAs (Section 5.3) provide a more robust, compact, low-cost and fast, alternative to LCTFs, providing a promising solution for multispectral endoscopic imaging (Translational Characteristic 2). However, it is currently unclear whether multispectral imaging can be used to delineate healthy pituitary tissue from pituitary adenoma, so in order to evaluate the appearance of pituitary adenoma and healthy pituitary tissue on multispectral endoscopy, a rigid multispectral endoscope was built to capture in vivo data in a pilot clinical study. Here, the design and development of this clinically translatable SRDA-based rigid multispectral endoscope is presented. Although clinical measurement has not yet been achieved, the initial technical characterisation is described and the challenges that must be addressed in order to achieve clinical translation are discussed.

\subsection{Endoscope Design}

In flexible endoscopy (Chapters 3-5), light is carried to the back end optics using long flexible fibres, so the back end optics can easily be spatially isolated from the patient, allowing great flexibility in their design. In transsphenoidal endoscopy, the back-end optics are clipped directly onto the rigid endoscope and handheld by the surgeon, so they must be compact and robust (Translational Characteristic 2). We thus designed a system based around a compact SRDA (CMS-V, SILIOS, France) (Figure 6.2).

Briefly, the system consists of a $4 \mathrm{~mm} 0^{\circ}$ endoscope (Sharpsite ${ }^{\circledR}$ AC Autoclavable Endoscope, Medtronics, USA) coupled to an SRDA (CMS-V, SILIOS, France) using a zoom coupler (18$35 \mathrm{~mm}$ zoom coupler, RVA Synergies, UK). The SRDA consists of 9 spectral filters (8 narrow bands; average FWHM 30 nm; centre wavelengths 553, 587, 629, 665, 714, 749, 791, 829 nm; 1 broad band; $500-850 \mathrm{~nm}$ ), deposited as a $3 \times 3$ super-pixel across a CMOS sensor (NIR Ruby sensor, UI1242LE-NIR, IDS, square pixel size $5.3 \mu \mathrm{m}$ ). Illumination is provided by a broadband ultra-high power LED (T7359, Prizmatix, Israel) coupled to the endoscope using an achromatic doublet lens (AC254-030-A, Thorlabs, Germany) housed inside a custom coupler with a smooth bore for a fibre optic light guide (495 NA, Karl Storz, Germany). Images were captured in uEye Cockpit (IDS, Germany) and saved as 8-bit BMP files. Data analysis was carried out using Matlab® (MathWorks, USA).

Similar to the flexible endoscopes presented in Chapters 3-5, this device architecture ensures that no modification has been made to any part of the endoscope that is intended to be in direct patient contact (Figure 6.2 (b)), meaning approval for use in humans can be granted locally by clinical engineering (Translational Characteristic 2). The back end optics are easily clipped on and off the rigid endoscope using a slider, ensuring minimal interruption to standard clinical 
workflow (Translational Characteristic 2). Since they are adjacent to the patient, the back end optics are covered with a plastic bag to keep them clean during surgery, as is standard protocol for rigid endoscopy cameras. The illumination is spatially isolated from the patient as it is supplied through a flexible light guide (Figure 6.2 (c)).

The multispectral data can be used to produce a false colour RGB image familiar to the surgeon, such that the surgeon can continue to survey the patient during multispectral imaging, watching for adverse events such as bleeding, thus ensuring the patient's safety is not compromised during this time (Translational Characteristic 6).

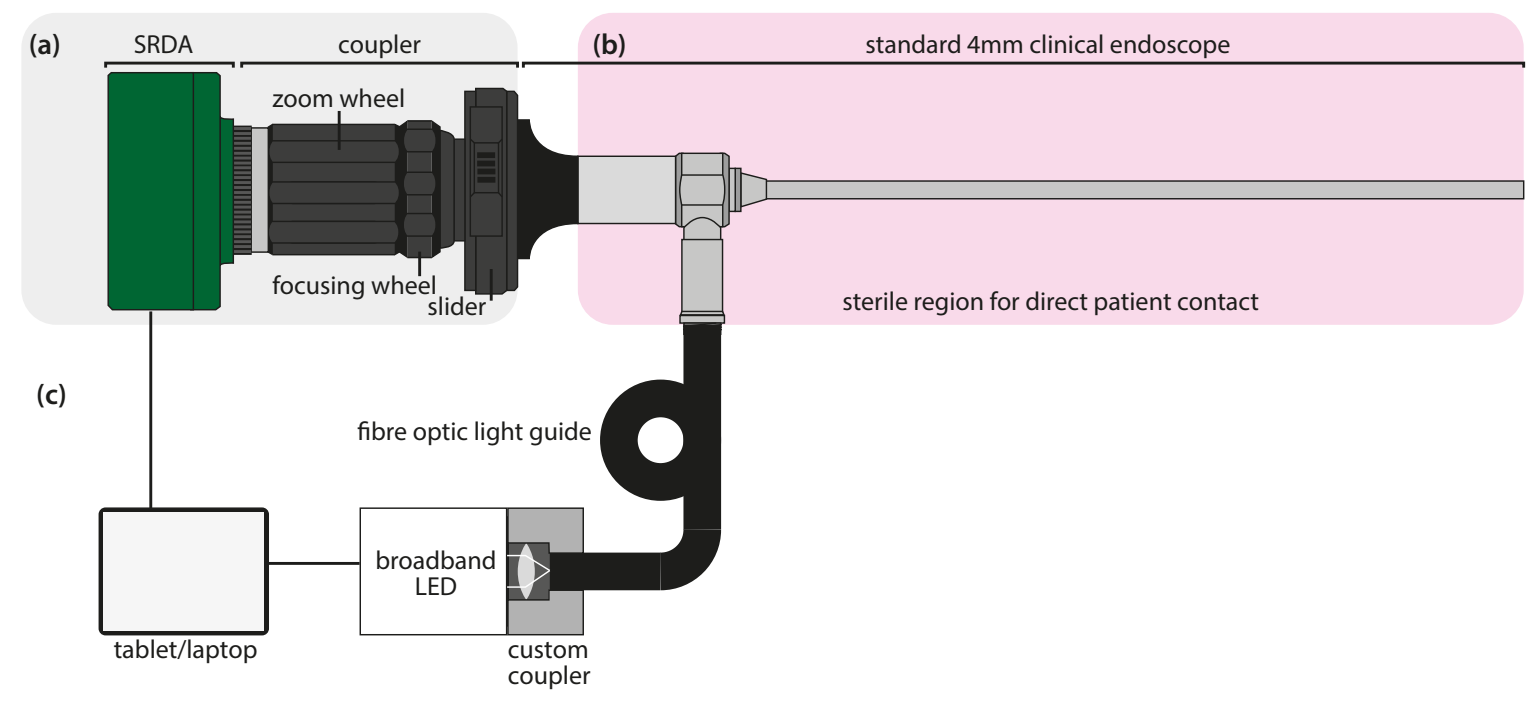

Figure 6.2 Schematic of the SRDA-based rigid multispectral endoscope.

(a) The back end optics are based around a compact and robust SRDA (CMS-V, SILIOS, France) coupled to the rigid endoscope using a commercially available camera coupler (18-35 mm zoom coupler, RVA Synergies, UK). They are easily clipped on and off of the rigid endoscope using a slider, ensuring minimal interruption to the standard clinical workflow. To ensure they remain clean during surgery, they are covered with a plastic bag. This is standard procedure for rigid endoscopy cameras. (b) The region in direct patient contact. (c) Illumination is provided by a broadband ultra-high power LED (T7359, Prizmatix, Israel) coupled to the endoscope via a custom coupler and a fibre optic light cable. The illumination is spatially isolated from the patient as it is passed through a flexible light guide. 


\subsection{Technical Characterisation}

Following design and construction of the SRDA-based rigid multispectral endoscope, technical characterisation of the system commenced.

\subsubsection{Methods}

\section{A. Resolution}

To determine the limiting resolution of the SRDA-based rigid multispectral endoscope, images of a 1951 USAF resolution test target (\#53-714, Edmund Optics, USA) were captured at 6 working distances $(7.5-20.0 \mathrm{~mm})$ using external illumination from a broadband halogen light source (OSL2B2, Thorlabs, Germany) to reduce specular reflections. The raw images were simply demosaicked as described in Section 3.3.4 and the broad band (500-850 $\mathrm{nm}$ ) channel images were analysed.

\section{B. Field of view}

In order to measure the field of view (FOV), images of a $1 \mathrm{~mm}$ checkerboard printed on white paper were captured at 17 working distances (WD), 4-20 mm (error $\pm 0.1 \mathrm{~mm}$ ). The resulting images are expected to show barrel distortion defined by:

$$
r_{u}=A r_{d}\left(1+k r_{d}^{2}\right)
$$

where $r_{u}$ is the radial distance from the center of the ground truth image to a given vertex $i$ in $\mathrm{mm}, r_{d}$ is the radial distance from the centre of the distorted image to the same vertex $i$ in pixels, $k$ is a constant that describes the magnitude of the distortion and $A$ is a constant used to convert between units of pixels and $\mathrm{mm}$.

For each of the images acquired the position of the checkerboard vertices were identified using the built in Matlab function 'detectCheckerboardPoints'. For each of these points, the radial distance to the centre of the image, $r_{d}$ (in pixels), and the true distance to the centre of the image, $r_{u}$ (in $\mathrm{mm}$ ), which is known from the checkerboard pattern, were found.

\subsubsection{Results}

\section{A. Resolution}

Resolution was determined by taking images of a USAF test target. The raw images were demosaicked and the broad band (500-850 nm) channel images were analysed. The Michelson contrast (Eq. 3.17) was calculated for each element and the results plotted against the reciprocal 
of the line width of the element (Figure 6.3). A contrast threshold of $1 \%$ has previously been reported to be applicable across a wide range of targets and conditions [198], but a threshold of $5 \%$ was chosen to avoid effects arising from noise at very low contrast. By finding the intersect of this threshold with exponential fits applied to the data at each WD, the resolution of the endoscope was determined to be $68 \pm 7,83 \pm 7,103 \pm 9,110 \pm 10,130 \pm 10$ and $150 \pm 10 \mu \mathrm{m}$, at working distances of 7.5, 10.0, 12.5, 15.0, 17.5 and $20 \mathrm{~mm}$ respectively.

\section{B. Field of view}

Example images displaying barrel distortion can be seen in Figure 6.4. The distortion constant $k$ and the constant $A$ were determined by fitting Eq. 6.1 to the data $\left(\mathrm{R}^{2}=0.9773-0.9927\right)$. The values of $k$ and $A$ were then used to determine the FOV radius $\left(=r_{u}\right)$ based on the radius of the images in pixels $\left(=r_{d}\right)$. Combining these data for the 20 working distances, the angle of the FOV was determined to be $96.6 \pm 0.6^{\circ}$, which compares favourably to the manufacturer specified angle of $102^{\circ}$.

\subsection{Clinical Trial}

Having developed a clinically translatable rigid multispectral endoscope, and having performed preliminary technical characterisation, a pilot cohort study was designed, entitled: "Prospective pilot cohort study to assess feasibility of multispectral endoscopic imaging for delineation of the pituitary gland during transphenoidal surgery for pituitary adenomas" or "Multispectral imaging of adenoma in pituitary surgery (MAPS)" for short. The aim is to recruit 20 patients for investigation in this study, with multispectral imaging occurring as part of the subjects' clinically indicated surgery.

\subsubsection{Methods}

\section{A. Trial objectives}

The trial is designed to address one primary objective and one secondary objective:

i. Evaluate the appearance of healthy pituitary tissue and pituitary adenoma at multispectral endoscopy to build a library of useful distinguishing characteristics since these are currently unknown and not easily investigated using ex vivo tissue.

ii. Assess the feasibility of imaging with the novel multispectral endoscope in endoscopic transphenoidal pituitary surgery. 


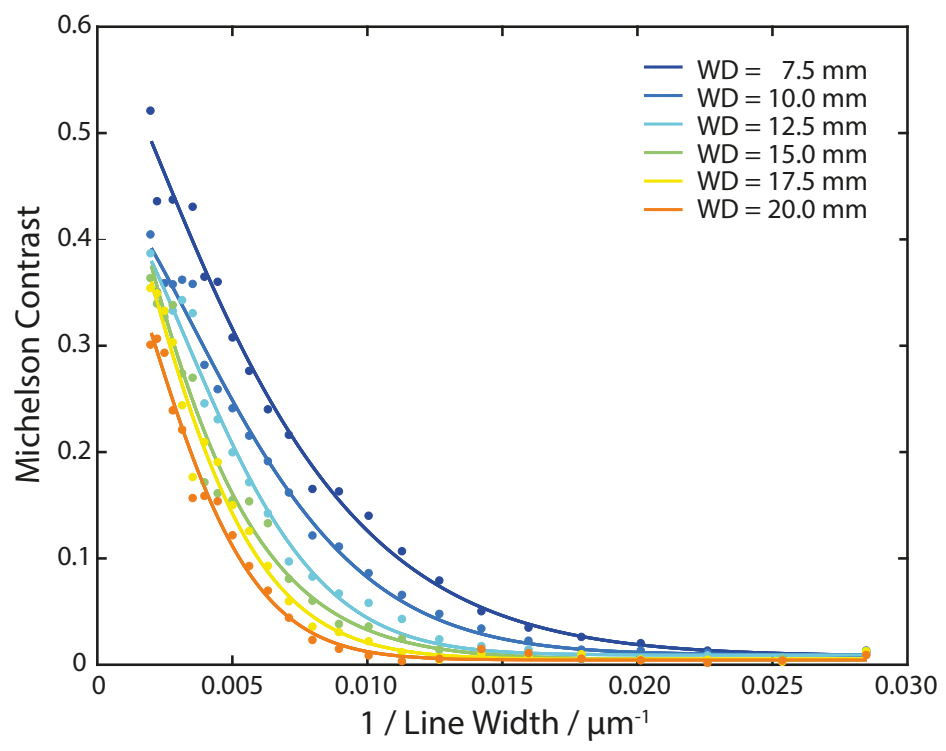

Figure 6.3 Characterisation of the resolution of the rigid multispectral endoscope.

Resolution was characterised by imaging a USAF chart at six different working distances. The resolution was determined as the point where an exponential fit drops below 5\% Michelson contrast. $\mathrm{R}^{2}=0.9913$, $0.9909,0.9896,0.9785,0.9862$ and 0.9886 for working distances of $7.5,10.0,12.5,15.0,17.5$ and $20 \mathrm{~mm}$ respectively.

(a)

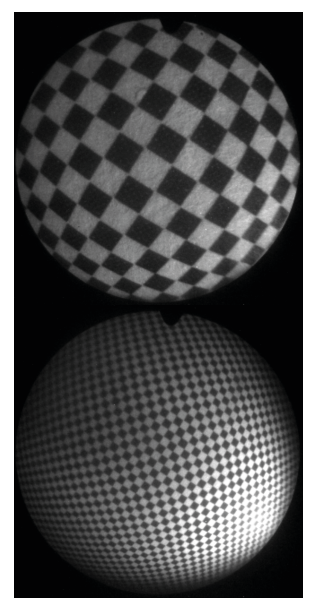

(b)

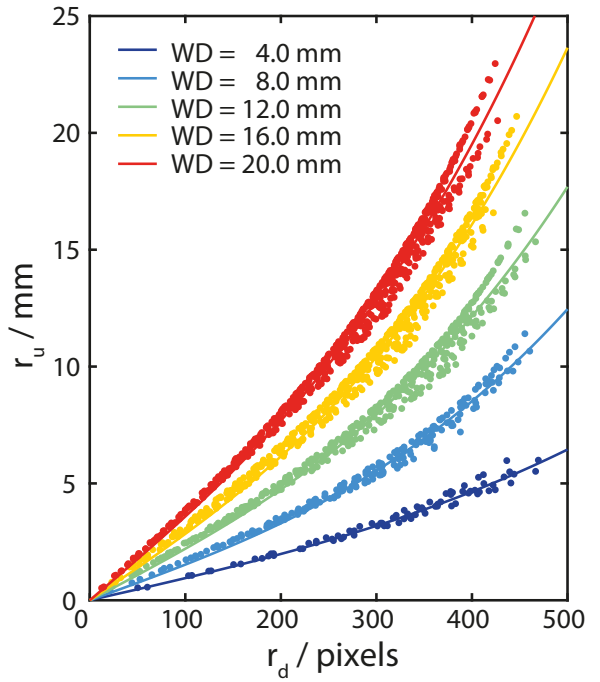

(c)

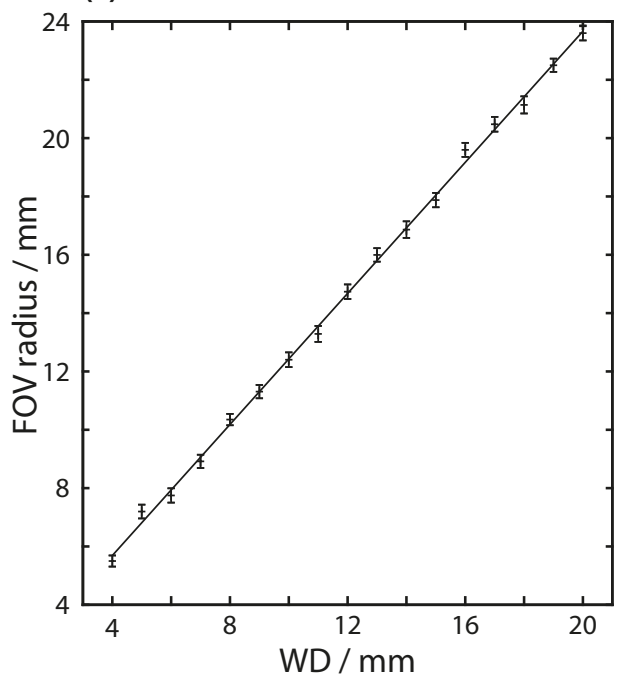

Figure 6.4 Characterisation of the FOV of the rigid multispectral endoscope.

(a) Images of $1 \mathrm{~mm}$ checkerboard paper at WD = 4 and $20 \mathrm{~mm}$ show barrel distortion. From such an image, $r_{u}$ and $r_{d}$ can be measured for several vertices on the paper. (b) Examples of the fit to Eq. 6.1 for images taken at a working distances (WD) of 4, 8, 12, 16 and $20 \mathrm{~mm}\left(\mathrm{R}^{2}=0.9799-0.9908\right)$. The fit was used to extract the constant $A$ and the distortion parameter $k$. The values of $A$ and $k$ can be used with Eq. 6.1 to determine the FOV radius $\left(=r_{u}\right)$ based on the radius of the images in pixels $\left(=r_{d}\right)$. (c) Determined FOV for 17 WDs $\left(\mathrm{R}^{2}=0.9986\right)$. Error bars represent the standard error of the FOV radius derived from the standard errors of the fit parameters $A$ and $k$. From the fitted line, the angular FOV was calculated to be $96.6 \pm 0.6^{\circ}$. 


\section{B. Trial design}

i. Potential study participants will be approached in clinic after they have agreed to pituitary surgery for their pituitary tumours. The study will be explained and patient information given with time for reflection before consent is taken to enter the study.

ii. On the day of surgery, nothing will be changed in terms of patient preparation for their operation. Their pituitary surgery will proceed with endoscopic resection of their pituitary tumour using a standard two surgeon four handed technique. During tumour resection (a. before the pituitary is seen, b. when the pituitary gland is thought to be seen and c. when the tumour resection has finished) the surgeon will take a still picture with the standard white light endoscope for orientation, before removing the standard camera from the end of the endoscope and replacing this with the multispectral camera for two minutes in order to capture intraoperative images.

iii. As is standard practice during endoscopic pituitary surgery, the tumour will be sent for histopathological analysis.

Images captured with the multispectral endoscope will be used to generate false colour images by assigning the narrow bands centred at 629,587 and $553 \mathrm{~nm}$ to RGB channels respectively. Following completion of the surgery, the surgeon will annotate these images with regions of interest where they are certain of the presence of particular tissue types such as healthy pituitary gland and pituitary adenoma. These regions of interest will be used to determine the multispectral signatures of the different tissue types.

\subsubsection{Results}

The trial was approved on $17^{\text {th }}$ August 2018 (Appendix B). A first patient is due to be recruited in late October 2018 (MAPS 01). 


\subsection{Conclusions and Future Work}

To address the clinical need for better visualisation of adenoma in pituitary surgery, an SRDAbased rigid multispectral endoscope capable of acquisition of 9-band multispectral images in vivo, with a resolution of $68 \pm 7 \mu \mathrm{m}$, and a FOV of $96.6 \pm 0.6^{\circ}$ was developed. Rigid SRDA-based multispectral endoscopy shares many of the same challenges of flexible SRDA-based multispectral endoscopy discussed in Chapter 5. SRDA-based multispectral imaging is limited in sensitivity and it remains to be seen whether this will hinder the use of the device in vivo during pituitary surgery. The data processing challenges discussed previously remain here. The rigid endoscope does not introduce a comb artefact as seen in flexible endoscopy. Whilst this affords a greater resolution ( $83 \pm 7$ vs. $240 \pm 20 \mu \mathrm{m}$ at $\mathrm{WD}=1 \mathrm{~cm}$ for the flexible and rigid SRDAbased multispectral endoscopes respectively), it comes at the price of increased processing time, since every pixel must be spectrally unmixed (not just those at fibre centres). For future realtime unmixing, shorter processing times might be achieved by utilising GPUs, or reducing the number of spectral bands.

Despite these challenges, the SRDA-based rigid multispectral endoscope has several advantages: it has received local safety approval for use in humans; the back end optics are easily clipped on and off a rigid endoscope using a slider, ensuring minimal interruption to standard clinical workflow (Translational Characteristic 2); it can be operated in the same way as familiar standard of care endoscopes (Translational Characteristic 3) and the multispectral data can be used to produce a false colour RGB image more familiar to the surgeon, ensuring that the patient's safety is not compromised during multispectral imaging (Translational Characteristic 6). Furthermore, MSI occurs directly on the SRDA, meaning the compact device has fewer optical components, and is more resistant to misalignment than alternative multispectral imaging techniques, which is especially important when the device must be handheld as is necessary in pituitary surgery (Translational Characteristic 2).

Having developed this device, we performed preliminary technical characterisation. This work represents the first steps in Domain 2 of the OIB Roadmap (Figure 1.3). For further translation, detailed technical validation should be carried out to ensure precision of the technique, and detailed biological validation should be performed, to relate images to underlying pathology. It is not possible to perform these experiments prior to understanding the specific spectral signatures that will ultimately be of interest, and assessment of these is not possible using ex vivo tissue for the reasons discussed previously (Section 5.7) (Translational Barrier 4). Therefore, a clinical pilot study (MAPS) to investigate the appearance of pituitary adenoma and healthy pituitary tissue on multispectral imaging was planned. 
The first patient is due to be recruited in late October 2018 (MAPS 01). Over the next 12 months, we aim to recruit 10-20 patients in MAPS, iteratively improving the device, methodology and analysis as we proceed. The captured images will be used to determine the spectral signatures of different tissue types, particularly healthy tissue and pituitary adenoma. The device will be modified to optimise precise and repeatable imaging of these spectra. Finally, using these as endmembers, we plan to unmix the multispectral images, to produce maps of the surgical field where different tissue types are clearly delineated. If this is successfully achieved, the next step would be to progress along the OIB Roadmap, carrying out a second clinical trial to assess the clinical utility of these maps. 


\section{CONCLUSIONS AND OUTLOOK}

This thesis establishes three optical imaging techniques based on two endoscopic device architectures to address unmet clinical needs in two indications: detection of dysplasia in endoscopic surveillance of Barrett's oesophagus; and delineation of pituitary adenomas in transphenoidal endoscopic surgery. In establishing these techniques, close attention was paid to the translational characteristics (Chapter 1) to ensure these techniques were appropriate for translation. The first, a flexible endoscope for oesophageal imaging (Chapter 3), was paired with NIR fluorescence optical molecular imaging (Chapter 4) and later SRDA-based multispectral imaging (Chapter 5) for detection of dysplasia in Barrett's oesophagus. The second, a rigid endoscope for intraoperative imaging during transphenoidal surgery, was paired with SRDA-based multispectral imaging for intraoperative delineation of pituitary adenoma (Chapter 6). In addition to the design of the devices, and the development of software for their control, correction methods were established to remove the comb structure associated with the fibre bundle of the flexible endoscopes, and to demosaic the images acquired with SRDAs (Chapter 3). Each of these three techniques was validated and compared with gold standards on measurements across a broad range of biological and non-biological samples. Finally, the two multispectral techniques were translated to in vivo clinical trials.

To ensure swift clinical translation of novel optical imaging techniques, the OIB Roadmap was developed, outlining the steps from discovery of a novel OIB to its widespread implementation in healthcare (Chapter 1). Associated with this, 6 key translational characteristics were defined. Careful consideration of a technique's repeatability and reproducibility, contrast mechanism, instrumentation, operator expertise, co-registration and image reader expertise, can promote its progression along the roadmap. These characteristics influenced many of the decisions in designing the optical imaging techniques presented in this thesis, and ultimately meant that the 
techniques were suitable for clinical measurements within the 4 year time frame of the work presented in this thesis.

The first part of this thesis described the ongoing work to develop novel optical imaging techniques for detection of dysplasia in patients with Barrett's oesophagus (Chapter 2). For this work, an accessory channel endoscope device architecture was chosen to facilitate local safety approval of the devices (Chapter 3). The major drawback of this approach is the comb artefact introduced by the imaging fibre bundle, but this was addressed by the use of decombing algorithms, several of which were compared to ensure that, for a given application, the best decombing method can be chosen. This not only guided decisions for data processing in this work, but it could also be effectively applied across other fibrescopic imaging applications using different weightings with the presented results.

Having identified a translatable device architecture, we designed and validated a bimodal NIR fluorescence and WL reflectance endoscope for OMI of WGA-IR800 to delineate dysplasia in Barrett's oesophagus (Chapter 4). The ex vivo results showed an encouraging correlation between fluorescence signal intensities and histopathological outcome [201, 209, 271]. With the majority of the initial validation completed using ex vivo tissue, and the device prepared for application to be locally approved for use in humans, the next step in the OIB Roadmap was to perform first-in-human trials. Much of the groundwork for these trials has been laid, but in order to ensure the quality and safety of WGA-IR800, it must be synthesised under good manufacturing practice (GMP) conditions before it can be used in humans, which is currently prohibitively expensive and therefore, was not possible in the timeframe of the work presented in this thesis.

As the first-in-human trials are yet to take place, it is currently unclear how well this technique will perform in vivo. As discussed in Chapter 4, some instrumentation challenges remain; fluorescence images ideally need to be corrected for variable working distance and the signal to noise ratio will likely need to be improved depending on the final bound concentration of WGA-IR800. The next step will be to apply the technique in vivo to assess the scale of these challenges. Work to find an inexpensive way to synthesise WGA-IR800 in GMP conditions, so that it may be applied in vivo, is ongoing. If this is achieved, a clinical pilot study of OMI using the bimodal endoscope is set to begin.

Due to the demonstrated potential of MSI in cancer detection, an SRDA-based multispectral endoscope was developed (Chapter 5). Initially, this device was applied to multispectral fluorescence imaging, demonstrating the ability to accurately detect fluorescent contrast agents, both in well plates, and in a realistic clinical scenario, using a whole ex vivo porcine oesophagus. The most significant challenge facing SRDA-based multispectral imaging was found to be the 
limited sensitivity of the device imposed by low sensitivity of the SRDA. This is important for real time imaging, especially in scenarios where signal is low, for example, when imaging low concentrations of fluorophore. Fabrication of custom sensors, for example by depositing the CFA on a higher QE sensor or miniaturising SRDAs and placing them on the tip of the endoscope, have the potential to increase sensitivity, but these require significant investment in research and development. Prior to these sensors being realised, further work on OMI would be likely to deploy the higher sensitivity bimodal system described in Chapter 4. In the meantime, we await the GMP synthesis and approval of a molecular contrast agent for use in humans.

Having already developed and characterised an SRDA-based multispectral endoscope, and given the potential of multispectral imaging to detect endogenous contrast, we modified the multispectral endoscope for reflectance imaging of endogenous reflectance. When tissue is excised from the body, endogenous tissue features change irreversibly (Translational Barrier 4), so technical and biological validation of multispectral reflectance imaging is not easily performed using ex vivo tissue, hence in vivo evaluation is required. Therefore, an exploratory pilot clinical study to assess the feasibility of multispectral endoscopic imaging for detection of dysplasia in patients with Barrett's oesophagus was planned (MuSE).

In the first trial of this pilot study, MuSE 01, multispectral endoscopy produced promising results. Distinct reflection spectra were observed for regions of Barrett's oesophagus and cancer, suggesting the potential to delineate diseased tissue based on endogenous reflectance using the multispectral endoscope. These spectra were demonstrated to be consistent over multiple viewpoints separated by several minutes of imaging, suggesting high repeatability of this approach.

Our work on flexible endoscopy, detailed in Chapters 2-5, established a framework for swiftly translating novel flexible endoscopic techniques to in vivo clinical pilot studies. It was shown that the PolyScope accessory channel endoscope is a useful device architecture for translating novel contrast mechanisms into clinical trials. Effective decombing algorithms necessary to overcome the comb artefact introduced by the PolyScope were developed and evaluated. A protocol for performing simulated endoscopy that can be executed in the Department of Physics, adjacent to our optics labs, was established using whole ex vivo porcine oesophagus, allowing new techniques to be tested in a realistic setting during the development phase, without the need for upheaval and transport to a clinic, where entry, time and space restrictions may prohibit effective early stage validation. Safety approval for PolyScope-based devices has been granted, this being sufficiently broad to cover alternative back end optics, enabling device modifications to be made without delays associated with seeking new or amended approval. Based on this 
framework, several novel endoscopic imaging techniques, including a hyperspectral endoscope and a phase sensitive endoscope, will be applied in vivo in the next few years.

Following the promising initial results of SRDA-based multispectral imaging in Barrett's, we aimed to apply this technique to intraoperative imaging during transsphenoidal surgery for resection of pituitary adenoma (Chapter 6). For this work. a rigid SRDA-based multispectral endoscope was constructed, preliminary technical characterisation was performed and a pilot clinical study (MAPS) was designed and accepted. The first patient is due to be recruited in late October 2018.

Over the next 12 months, we aim to recruit 10-20 patients in both the MuSE and the MAPS studies, iteratively improving the devices, methodology and analysis as the trials proceed. The captured images will be used to build a database of tissue spectral properties from which the spectral signatures of different tissue types, Barrett's oesophagus vs. dysplasia and healthy pituitary vs. adenoma, can be determined. Using these spectral signatures as endmembers, images clearly delineating different tissue types could be produced. The next step would be to carry out prospective clinical trials to determine the clinical utility of these techniques, crossing Translational Gap 1 in the OIB Roadmap. Were their clinical utility proven in multicentre clinical trials, these techniques might one day cross Translational Gap 2 to be incorporated into routine healthcare, improving upon the current standard of care. 


\section{REFERENCES}

[1] Wax, A., Terry, N. G., Dellon, E. S. \& Shaheen, N. J. Angle-resolved low coherence interferometry for detection of dysplasia in Barrett's esophagus. Gastroenterology 141, 443-447 (2011).

[2] Bigio, I. J. \& Fantini, S. Quantitative Biomedical Optics. (Cambridge University Press, 2016).

[3] Imamoto, Y. \& Shichida, Y. Cone visual pigments. Biochimica et Biophysica Acta Bioenergetics 1837, 664-673 (2014).

[4] O'Connor, J. P. B. et al. Imaging biomarker roadmap for cancer studies. Nature Reviews Clinical Oncology 14, 169-186 (2017).

[5] Hanahan, D. \& Weinberg, R. a. Hallmarks of cancer: the next generation. Cell 144, 64674 (2011).

[6] Bhat, Y. M. et al. High-definition and high-magnification endoscopes. Gastrointestinal Endoscopy 80, 919-927 (2014).

[7] Rameshshanker, R. \& Wilson, A. Electronic Imaging in Colonoscopy: Clinical Applications and Future Prospects. Current Treatment Options in Gastroenterology 14, 140-151 (2016).

[8] Manfredi, M. A. et al. Electronic chromoendoscopy. Gastrointestinal Endoscopy 81, 249-261 (2015).

[9] Thosani, N. et al. ASGE Technology Committee systematic review and meta-analysis assessing the ASGE Preservation and Incorporation of Valuable Endoscopic Innovations thresholds for adopting real-time imaging-assisted endoscopic targeted biopsy during endoscopic surveillance. Gastrointestinal Endoscopy 83, 684-698 (2016).

[10] Lu, G. \& Fei, B. Medical hyperspectral imaging: a review. Journal of biomedical optics 19, 10901 (2014).

[11] Jang, J. The Past, Present, and Future of Image-Enhanced Endoscopy. 466-475 (2015).

[12] Olympus -Technologies NBI| Medical Systems. Available at: https://www.olympuseuropa.com/medical/en/medical_systems/technologies/narrow_band_imaging_nbi_1/t echnologies_nbi.jsp. (Accessed: 26th April 2017)

[13] FICE Dual Mode | Fujifilm Europe. Available at: https://www.fujifilm.eu/eu/products/medical-systems/endoscopy/technology/fice-dualmode. (Accessed: 26th April 2017)

[14] Advanced Imaging | PENTAX Medical (EMEA). Available at: https://www.pentaxmedical.com/pentax/en/95/1/WavSTAT4-Optical-Biopsy-System. (Accessed: 26th April 2017)

[15] MedX Health - About SIMSYS-MoleMate ${ }^{\mathrm{TM}}$. Available at: http://medxhealth.com/OurProducts/SIAscopytrade;/overview.aspx. (Accessed: 26th April 2017)

[16] MelaFind. Available at: http://www.melafind.com/melafind/. (Accessed: 26th April 2017)

[17] Spectral Molecular Imaging - Products. Available at: http://www.opmol.com/products.html. (Accessed: 26th April 2017)

[18] Alali, S. \& Vitkin, A. Polarized light imaging in biomedicine: emerging Mueller matrix methodologies for bulk tissue assessment. Journal of Biomedical Optics 20, 061104 
(2015).

[19] [DERMA MEDICAL SYSTEMS] English. Available at: https://www.dermamedicalsystems.com/index.php?menu_id=117. (Accessed: 2nd May 2017)

[20] Sturm, M. B. \& Wang, T. D. Emerging optical methods for surveillance of Barrett's oesophagus. Gut 64, 1816-1823 (2015).

[21] James, M. L. \& Gambhir, S. S. A Molecular Imaging Primer: Modalities, Imaging Agents, and Applications. Physiol. Rev. 92, 897-965 (2012).

[22] Ulrich, M. et al. Dynamic Optical Coherence Tomography in Dermatology. Dermatology 232, 298-311 (2016).

[23] Cellvizio: Our Flagship Product | Mauna Kea Technologies. Available at: http://www.maunakeatech.com/en/hospital-administrators/cellvizio-solution. (Accessed: 26th April 2017)

[24] ViewnVivo Home - ViewnVivo - Must-see, Miniaturised In Vivo Microscopy. Available at: http://viewnvivo.com/. (Accessed: 26th April 2017)

[25] Caliber I.D. - Clinical Applications. Available at: http://www.caliberid.com/clinical.html. (Accessed: 2nd May 2017)

[26] Wong Kee Song, L. M. et al. Autofluorescence imaging. Gastrointestinal Endoscopy 73, 647-650 (2011).

[27] Hanson, K. M. \& Bardeen, C. J. Application of nonlinear optical microscopy for imaging skin. Photochemistry and Photobiology 85, 33-44 (2009).

[28] Fu, L. \& Gu, M. Fibre-optic nonlinear optical microscopy and endoscopy. Journal of Microscopy 226, 195-206 (2007).

[29] Thomas, G., Van Voskuilen, J., Gerritsen, H. C. \& Sterenborg, H. J. C. M. Advances and challenges in label-free nonlinear optical imaging using two-photon excitation fluorescence and second harmonic generation for cancer research. Journal of Photochemistry and Photobiology B: Biology 141, 128-138 (2014).

[30] Jenlab: DermaInspect. Available at: http://www.jenlab.de/DermaInspect.29.0.html. (Accessed: 5th May 2017)

[31] Trindade, A. J., Smith, M. S. \& Pleskow, D. K. The new kid on the block for advanced imaging in Barrett's esophagus: a review of volumetric laser endomicroscopy. Therapeutic advances in gastroenterology 9, 408-16 (2016).

[32] NinePoint Medical. Available at: http://www.ninepointmedical.com/\#NvisionVLE. (Accessed: 26th April 2017)

[33] Verisante Technology, Inc. Available at: http://www.verisante.com/products/core/. (Accessed: 26th April 2017)

[34] Boone, M. A. L. M., Norrenberg, S., Jemec, G. B. E. \& Del Marmol, V. High-definition optical coherence tomography imaging of melanocytic lesions: A pilot study. Archives of Dermatological Research 306, 11-26 (2014).

[35] Product - Vivosight. Available at: https://vivosight.com/about-us/product/. (Accessed: 28th April 2017)

[36] Kallaway, C. et al. Advances in the clinical application of Raman spectroscopy for cancer diagnostics. Photodiagnosis and Photodynamic Therapy 10, 207-219 (2013).

[37] Pence, I. \& Mahadevan-Jansen, A. Clinical instrumentation and applications of Raman spectroscopy. Chem. Soc. Rev. 45, 1958-1979 (2016). 
[38] Wang, W., Zhao, J., Short, M. \& Zeng, H. Real-time in vivo cancer diagnosis using raman spectroscopy. Journal of Biophotonics 8, 527-545 (2015).

[39] Tu, Q. \& Chang, C. Diagnostic applications of Raman spectroscopy. Nanomedicine: Nanotechnology, Biology, and Medicine 8, 545-558 (2012).

[40] Verisante Aura ${ }^{\mathrm{TM}}$. Available at: http://www.verisante.com/aura/medical_professional/. (Accessed: 26th April 2017)

[41] Benes, Z. \& Antos, Z. Optical biopsy system distinguishing between hyperplastic and adenomatous polyps in the colon during colonoscopy. Anticancer Research 29, 47374739 (2009).

[42] Boerwinkel, D. F. et al. Fluorescence spectroscopy incorporated in an Optical Biopsy System for the detection of early neoplasia in Barrett's esophagus. Diseases of the esophagus : official journal of the International Society for Diseases of the Esophagus / I.S.D.E (2014).

[43] Grosenick, D., Rinneberg, H., Cubeddu, R. \& Taroni, P. Review of optical breast imaging and spectroscopy. Journal of Biomedical Optics 21, 091311 (2016).

[44] Yu, G. Near-infrared diffuse correlation spectroscopy in cancer diagnosis and therapy monitoring. Journal of Biomedical Optics 17, 010901 (2012).

[45] Sharma, P. et al. White Paper AGA: Advanced Imaging in Barrett's Esophagus. Clinical Gastroenterology and Hepatology 13, 2209-2218 (2015).

[46] ICNIRP. ICNIRP guidelines on limits of exposure to incoherent visible and infrared radiation. Health Physics 71, 804-819 (2013).

[47] Brookner, C. K., Agrawal, A., Trujillo, E. V., Mitchell, M. F. \& Richards-Kortum, R. R. Safety Analysis: Relative Risks of Ultraviolet Exposure from Fluorescence Spectroscopy and Colposcopy Are Comparable. Photochemistry and Photobiology 65, 1020-1025 (1997).

[48] DIRECTIVE 2006/25/EC OF THE EUROPEAN PARLIAMENT AND OF THE COUNCIL. (2006).

[49] NEMA. Performance Measurements of Positron Emission Tomographs (PETs). (2013). Available at: https://www.nema.org/Standards/Pages/Performance-Measurements-ofPositron-Emission-Tomographs.aspx.

[50] Tummers, W. S. et al. Regulatory aspects of optical methods and exogenous targets for cancer detection. Cancer Research 77, 2197-2206 (2017).

[51] Van Norman, G. A. Drugs and Devices: Comparison of European and U.S. Approval Processes. JACC: Basic to Translational Science 1, 399-412 (2016).

[52] Van Norman, G. A. Drugs, Devices, and the FDA: Part 1. JACC: Basic to Translational Science 1, 170-179 (2016).

[53] Downs-Kelly, E. et al. Poor interobserver agreement in the distinction of high-grade dysplasia and adenocarcinoma in pretreatment Barrett's esophagus biopsies. The American journal of gastroenterology 103, 2333-40; quiz 2341 (2008).

[54] O'Connor, J. P. B. et al. Imaging intratumor heterogeneity: Role in therapy response, resistance, and clinical outcome. Clinical Cancer Research 21, 249-257 (2015).

[55] Shim, M. G. \& Wilson, B. C. The effects of ex vivo handling procedures on the nearinfrared Raman spectra of normal mammalian tissues. Photochemistry and photobiology 63, 662-671 (1996).

[56] Usher-Smith, J. A., Sharp, S. J. \& Griffin, S. J. The spectrum effect in tests for risk 
prediction, screening, and diagnosis. BMJ i3139 (2016).

[57] Monheit, G. et al. The performance of MelaFind: a prospective multicenter study. Archives of dermatology 147, 188-94 (2011).

[58] Silva, F. B. et al. Endoscopic assessment and grading of Barrett's esophagus using magnification endoscopy and narrow-band imaging: Accuracy and interobserver agreement of different classification systems (with videos). Gastrointestinal Endoscopy 73, 7-14 (2011).

[59] Kara, M. a, Ennahachi, M., Fockens, P., ten Kate, F. J. W. \& Bergman, J. J. G. H. M. Detection and classification of the mucosal and vascular patterns (mucosal morphology) in Barrett's esophagus by using narrow band imaging. Gastrointestinal endoscopy 64, 155-66 (2006).

[60] Singh, R. et al. Narrow-band imaging with magnification in Barrett's esophagus: validation of a simplified grading system of mucosal morphology patterns against histology. Endoscopy 40, 457-63 (2008).

[61] Sharma, P. et al. The utility of a novel narrow band imaging endoscopy system in patients with Barrett's esophagus. Gastrointestinal Endoscopy 64, 167-175 (2006).

[62] Curvers, W. L. et al. Endoscopic tri-modal imaging is more effective than standard endoscopy in identifying early-stage neoplasia in Barrett's esophagus. Gastroenterology 139, 1106-14 (2010).

[63] Curvers, W. L. et al. Endoscopic trimodal imaging versus standard video endoscopy for detection of early Barrett's neoplasia: a multicenter, randomized, crossover study in general practice. Gastrointestinal endoscopy 73, 195-203 (2011).

[64] Trivedi, P. J. \& Braden, B. Indications, stains and techniques in chromoendoscopy. QJM : monthly journal of the Association of Physicians 106, 117-31 (2013).

[65] Sevick-Muraca, E. M. et al. Advancing the translation of optical imaging agents for clinical imaging. Biomedical Optics Express 4, 160-70 (2013).

[66] Overview of Device Regulation. Available at: https://www.fda.gov/MedicalDevices/DeviceRegulationandGuidance/Overview/default .htm. (Accessed: 27th November 2017)

[67] Medical devices - European Commission. Available at: https://ec.europa.eu/growth/sectors/medical-devices_en. (Accessed: 27th November 2017)

[68] Gora, M. J. et al. Tethered capsule endomicroscopy enables less invasive imaging of gastrointestinal tract microstructure. Nature medicine 19, 238-40 (2013).

[69] Sharma, P. et al. Development and Validation of a Classification System to Identify High-grade Dysplasia and Esophageal Adenocarcinoma in Barrett's Esophagus Using Narrow Band Imaging. Gastroenterology 150, 591-598 (2016).

[70] Boerwinkel, D. F. et al. Third-generation autofluorescence endoscopy for the detection of early neoplasia in Barrett's esophagus: a pilot study. Diseases of the esophagus: official journal of the International Society for Diseases of the Esophagus / I.S.D.E 27, 276-84 (2014).

[71] de Bruijne, M. Machine learning approaches in medical image analysis: From detection to diagnosis. Medical Image Analysis 33, 94-97 (2016).

[72] Suzuki, K. Overview of deep learning in medical imaging. Radiological Physics and Technology 10, 1-17 (2017).

[73] Garcia-Allende, P. B. et al. Towards clinically translatable NIR fluorescence molecular 
guidance for colonoscopy. Biomedical optics express 5, 78-92 (2013).

[74] Suter, M. J. et al. Esophageal-guided biopsy with volumetric laser endomicroscopy and laser cautery marking: A pilot clinical study. Gastrointestinal Endoscopy 79, 886-896 (2014).

[75] Pelargos, P. E. et al. Utilizing virtual and augmented reality for educational and clinical enhancements in neurosurgery. Journal of Clinical Neuroscience 35, 1-4 (2016).

[76] Nicolau, S., Soler, L., Mutter, D. \& Marescaux, J. Augmented reality in laparoscopic surgical oncology. Surgical Oncology 20, 189-201 (2011).

[77] CRUK. Resources | CRUK Cambridge Centre Early Detection Programme. Available at: https://www.earlydetectioncambridge.org.uk/resources. (Accessed: 18th January 2018)

[78] Gatenby, P. et al. Lifetime risk of esophageal adenocarcinoma in patients with Barrett's esophagus. World journal of gastroenterology: WJG 20, 9611-7 (2014).

[79] Desai, T. K. et al. The incidence of oesophageal adenocarcinoma in non-dysplastic Barrett's oesophagus: a meta-analysis. Gut 61, 970-6 (2012).

[80] Bhat, S. et al. Risk of malignant progression in Barrett's esophagus patients: results from a large population-based study. Journal of the National Cancer Institute 103, 1049-57 (2011).

[81] Hvid-Jensen, F., Pedersen, L., Drewes, A. M., Sørensen, H. T. \& Funch-Jensen, P. Incidence of adenocarcinoma among patients with Barrett's esophagus. The New England journal of medicine 365, 1375-83 (2011).

[82] Duits, L. C. et al. Barrett's oesophagus patients with low-grade dysplasia can be accurately risk-stratified after histological review by an expert pathology panel. Gut 64, 700-6 (2015).

[83] Reid, B. J., Levine, D. S., Longton, G., Blount, P. L. \& Rabinovitch, P. S. Predictors of progression to cancer in Barrett's esophagus: Baseline histology and flow cytometry identify low- and high-risk patient subsets. American Journal of Gastroenterology 95, 1669-1676 (2000).

[84] CRUK. Oesophageal cancer statistics. http://www.cancerresearchuk.org/healthprofessional/cancer-statistics/statistics-by-cancer-type/oesophageal-cancer

[85] Barbour, A. P. et al. Risk stratification for early esophageal adenocarcinoma: analysis of lymphatic spread and prognostic factors. Annals of surgical oncology 17, 2494-502 (2010).

[86] Fitzgerald, R. C. et al. British Society of Gastroenterology guidelines on the diagnosis and management of Barrett's oesophagus. Gut 63, 7-42 (2014).

[87] Weusten, B. L. A. M. et al. Endoscopic management of Barrett's esophagus: European Society of Gastrointestinal Endoscopy (ESGE) Position Statement. Endoscopy 191-198 (2017).

[88] Shaheen, N. J., Falk, G. W., Iyer, P. G., Gerson, L. B. \& American College of Gastroenterology. ACG Clinical Guideline: Diagnosis and Management of Barrett's Esophagus. The American Journal of Gastroenterology 111, 30-50 (2016).

[89] Evans, J. A. et al. The role of endoscopy in Barrett's esophagus and other premalignant conditions of the esophagus. Gastrointestinal Endoscopy 76, 1087-1094 (2012).

[90] Spechler, S. J., Sharma, P., Souza, R. F., Inadomi, J. M. \& Shaheen, N. J. American Gastroenterological Association technical review on the management of Barrett's esophagus. Gastroenterology 140, e18-e52 (2011). 
[91] Kastelein, F. et al. Impact of surveillance for Barrett's oesophagus on tumour stage and survival of patients with neoplastic progression. Gut 65, 1-7 (2015).

[92] Verbeek, R. E. et al. Surveillance of Barrett's Esophagus and Mortality from Esophageal Adenocarcinoma: A Population-Based Cohort Study. The American journal of gastroenterology 109, 1215-1222 (2014).

[93] El-Serag, H. B. et al. Surveillance endoscopy is associated with improved outcomes of oesophageal adenocarcinoma detected in patients with Barrett's oesophagus. Gut 65, 1252-1260 (2016).

[94] Corley, D. A. et al. Impact of endoscopic surveillance on mortality from Barrett's esophagus-associated esophageal adenocarcinomas. Gastroenterology 145, 312-319 (2013).

[95] Levine, D. S., Blount, P. L., Rudolph, R. E. \& Reid, B. J. Safety of a systematic endoscopic biopsy protocol in patients with Barrett's esophagus. The American journal of gastroenterology 95, 1152-7 (2000).

[96] Brown, H. et al. Scoping the future: An evaluation of endoscopy capacity across the NHS in England. (2015).

[97] Bergholt, M. S. et al. Fiberoptic confocal raman spectroscopy for real-time in vivo diagnosis of dysplasia in Barrett's esophagus. Gastroenterology 146, 27-32 (2014).

[98] Chedgy, F. J. Q., Subramaniam, S., Kandiah, K., Thayalasekaran, S. \& Bhandari, P. Acetic acid chromoendoscopy: Improving neoplasia detection in Barrett's esophagus. World Journal of Gastroenterology 22, 5753-5760 (2016).

[99] Beg, S., Wilson, A. \& Ragunath, K. The use of optical imaging techniques in the gastrointestinal tract. Frontline Gastroenterology 7, 207-215 (2016).

[100] Swager, A., Curvers, W. L. \& Bergman, J. J. Diagnosis by endoscopy and advanced imaging. Best Practice and Research: Clinical Gastroenterology 29, 97-111 (2015).

[101] Olliver, J. R., Wild, C. P., Sahay, P., Dexter, S. \& Hardie, L. J. Chromoendoscopy with methylene blue and associated DNA damage in Barrett's oesophagus. Lancet 362, 3734 (2003).

[102] Coletta, M. et al. Acetic acid chromoendoscopy for the diagnosis of early neoplasia and specialized intestinal metaplasia in Barrett's esophagus: A meta-analysis. Gastrointestinal Endoscopy 83, 57-67 (2016).

[103] Sharma, P. et al. Standard endoscopy with random biopsies versus narrow band imaging targeted biopsies in Barrett's oesophagus: a prospective, international, randomised controlled trial. Gut 62, 15-21 (2013).

[104] Maes, S., Sharma, P. \& Bisschops, R. Review: Surveillance of patients with Barrett oesophagus. Best Practice \& Research Clinical Gastroenterology 30, 901-912 (2016).

[105] Pohl, J. et al. Comparison of computed virtual chromoendoscopy and conventional chromoendoscopy with acetic acid for detection of neoplasia in Barrett's esophagus. Endoscopy 39, 594-8 (2007).

[106] Boerwinkel, D. F. et al. Effects of autofluorescence imaging on detection and treatment of early neoplasia in patients with Barrett's esophagus. Clinical Gastroenterology and Hepatology 12, 774-81 (2014).

[107] Giacchino, M. et al. Clinical utility and interobserver agreement of autofluorescence imaging and magnification narrow-band imaging for the evaluation of Barrett's esophagus: a prospective tandem study. Gastrointestinal endoscopy 77, 711-8 (2013).

[108] Sharma, P. et al. Real-time increased detection of neoplastic tissue in Barrett's esophagus 
with probe-based confocal laser endomicroscopy: final results of an international multicenter, prospective, randomized, controlled trial. Gastrointestinal endoscopy 74, 465-72 (2011).

[109] Trovato, C. et al. Confocal laser endomicroscopy for in vivo diagnosis of Barrett's oesophagus and associated neoplasia: a pilot study conducted in a single Italian centre. Digestive and Liver Disease 45, 396-402 (2013).

[110] Longcroft-Wheaton, G. et al. Duration of acetowhitening as a novel objective tool for diagnosing high risk neoplasia in Barrett's esophagus: A prospective cohort trial. Endoscopy 45, 426-432 (2013).

[111] Ngamruengphong, S., Sharma, V. K. \& Das, A. Diagnostic yield of methylene blue chromoendoscopy for detecting specialized intestinal metaplasia and dysplasia in Barrett's esophagus: a meta-analysis. Gastrointestinal endoscopy 69, 1021-8 (2009).

[112] Kaneko, K. et al. Effect of novel bright image enhanced endoscopy using blue laser imaging (BLI). Endoscopy International Open 02, E212-E219 (2014).

[113] Osawa, H. et al. Blue laser imaging provides excellent endoscopic images of upper gastrointestinal lesions. Video Journal and Encyclopedia of GI Endoscopy 1, 607-610 (2014).

[114] Miyake, Y. et al. Development of New Electronic Endoscopes Using the Spectral Images of an Internal Organ. in Proceedings of the IS\&T/SID's Thirteen Color Imaging Conference 261-269 (Society for Imaging Science and Technology, 2005).

[115] Kodashima, S. \& Fujishiro, M. Novel image-enhanced endoscopy with i-scan technology. World Journal of Gastroenterology 16, 1043-1049 (2010).

[116] Rodriguez, S. A. et al. Ultrathin endoscopes. Gastrointestinal Endoscopy 71, 893-898 (2010).

[117] Imagawa, H. et al. Improved visibility of lesions of the small intestine via capsule endoscopy with computed virtual chromoendoscopy. Gastrointestinal Endoscopy 73, 299-306 (2011).

[118] Dung, L. R. \& Wu, Y. Y. A wireless narrowband imaging chip for capsule endoscope. IEEE Transactions on Biomedical Circuits and Systems 4, 462-468 (2010).

[119] von Holstein, C. S. et al. Detection of adenocarcinoma in Barrett's oesophagus by means of laser induced fluorescence. Gut 39, 711-716 (1996).

[120] Kara, M. A. et al. Endoscopic video autofluorescence imaging may improve the detection of early neoplasia in patients with Barrett's esophagus. Gastrointestinal Endoscopy 61, 679-685 (2005).

[121] Wallace, M. et al. Miami classification for probe-based confocal laser endomicroscopy. Endoscopy 43, 882-91 (2011).

[122] Xiong, Y. Q., Ma, S. J., Zhou, J. H., Zhong, X. S. \& Chen, Q. A meta-analysis of confocal laser endomicroscopy for the detection of neoplasia in patients with Barrett's esophagus. Journal of Gastroenterology and Hepatology (Australia) 31, 1102-1110 (2016).

[123] Kandiah, K. et al. OC-054 Development and Validation of a Classification System to Identify Barrett's Neoplasia Using Acetic Acid Chromoendoscopy: The Predict Classification: Abstract OC-054 Table 1. Gut 65, A31.1-A31 (2016).

[124] Robles, L. Y., Singh, S. \& Fisichella, P. M. Emerging enhanced imaging technologies of the esophagus: spectroscopy, confocal laser endomicroscopy, and optical coherence tomography. Journal of Surgical Research 195, 502-514 (2015).

[125] Gora, M. J., Suter, M. J., Tearney, G. J. \& Li, X. Endoscopic optical coherence 
tomography: technologies and clinical applications [Invited]. Biomedical Optics Express 8, 2405 (2017).

[126] Leggett, C. L. et al. Comparative diagnostic performance of volumetric laser endomicroscopy and confocal laser endomicroscopy in the detection of dysplasia associated with Barrett's esophagus. Gastrointestinal Endoscopy 83, 880-888.e2 (2015).

[127] Trindade, A. J., George, B. J., Berkowitz, J., Sejpal, D. V \& McKinley, M. J. Volumetric laser endomicroscopy can target neoplasia not detected by conventional endoscopic measures in long segment Barrett's esophagus. Endoscopy international open 4, E31822 (2016).

[128] NvisionVLE® Imaging System - NinePoint Medical. Available at: http://www.ninepointmedical.com/nvisionvle-imaging-system/. (Accessed: 1st August 2017)

[129] Gora, M. J. et al. Imaging the upper gastrointestinal tract in unsedated patients using tethered capsule endomicroscopy. Gastroenterology 145, 723-725 (2013).

[130] Gora, M. et al. Tethered capsule endomicroscopy : from bench to bedside at a primary care practice Tethered capsule endomicroscopy : from bench to bedside at a primary care practice. Journal of Biomedical Optics 21, 104001 (2016).

[131] Swager, A. et al. Volumetric laser endomicroscopy in Barrett's esophagus: A feasibility study on histological correlation. Diseases of the Esophagus 1-8 (2015).

[132] Tsai, T. H. et al. Endoscopic optical coherence angiography enables 3-dimensional visualization of subsurface microvasculature. Gastroenterology 147, 1219-1221 (2014).

[133] Lee, H. C. et al. Endoscopic optical coherence tomography angiography microvascular features associated with dysplasia in Barrett's esophagus (with video). Gastrointestinal Endoscopy 86, 476-484 (2017).

[134] Ughi, G. J. et al. Automated segmentation and characterization of esophageal wall in vivo by tethered capsule optical coherence tomography endomicroscopy. 7, 660-665 (2016).

[135] Lovat, L. B. et al. Elastic scattering spectroscopy accurately detects high grade dysplasia and cancer in Barrett's oesophagus. Gut 55, 1078-83 (2006).

[136] Douplik, A. et al. Diffuse reflectance spectroscopy in Barrett's Esophagus: Developing a large field-of-view screening method discriminating dysplasia from metaplasia. Journal of Biophotonics 7, 304-311 (2014).

[137] Perelman, L. T. \& Backman, V. Light scattering spectroscopy of epithelial tissue: Principles and applications. in Handbook of Optical Biomedical Diagnostics (SPIE PRESS, 2016).

[138] Wallace, M. et al. Endoscopic detection of dysplasia in patients with Barrett\&apos;s esophagus using light-scattering spectroscopy. Gastroenterology 119, 677-682 (2000).

[139] Qiu, L. et al. Spectral Imaging With Scattered Light: From Early Cancer Detection to Cell Biology. IEEE Journal of Selected Topics in Quantum Electronics 18, 1073-1083 (2012).

[140] Lee, J. H. \& Wang, T. D. Molecular endoscopy for targeted imaging in the digestive tract. The Lancet Gastroenterology and Hepatology 1, 147-155 (2016).

[141] Terry, N. G. et al. Detection of dysplasia in Barrett's esophagus with in vivo depthresolved nuclear morphology measurements. Gastroenterology 140, 42-50 (2011).

[142] Yang, J.-M. et al. Three-dimensional photoacoustic and ultrasonic endoscopic imaging of two rabbit esophagi. Proceedings of SPIE 9323, (2015). 
[143] Pfefer, T. J., Paithankar, D. Y., Poneros, J. M., Schomacker, K. T. \& Nishioka, N. S. Temporally and spectrally resolved fluorescence spectroscopy for the detection of high grade dysplasia in Barrett's esophagus. Lasers in Surgery and Medicine 32, 10-16 (2003).

[144] Chen, J., Wong, S., Nathanson, M. H. \& Jain, D. Evaluation of Barrett esophagus by multiphoton microscopy. Archives of pathology \& laboratory medicine 138, 204-12 (2014).

[145] Joshi, B. P. et al. Multimodal endoscope can quantify wide-field fluorescence detection of Barrett's neoplasia. Endoscopy 48, (2016).

[146] Wolfsen, H. C. et al. Safety and feasibility of volumetric laser endomicroscopy in patients with Barrett's esophagus (with videos). Gastrointestinal Endoscopy 82, 631640 (2015).

[147] Kim, S. et al. Analyzing spatial correlations in tissue using angle-resolved low coherence interferometry measurements guided by co-located optical coherence tomography. Biomedical Optics Express 7, 1400 (2016).

[148] Qi, J. \& Elson, D. S. A high definition Mueller polarimetric endoscope for tissue characterisation. Scientific Reports 6, 25953 (2016).

[149] Ba, C., Palmiere, M., Ritt, J. \& Mertz, J. Dual-modality endomicroscopy with coregistered fluorescence and phase contrast. Biomedical Optics Express 7, 3403 (2016).

[150] Tan, A. C. S. et al. An overview of the clinical applications of optical coherence tomography angiography. Eye 1-25 (2017).

[151] Kashani, A. H. et al. Optical coherence tomography angiography: A comprehensive review of current methods and clinical applications. Progress in Retinal and Eye Research 60, 66-100 (2017).

[152] Wang, L. V \& Yao, J. A practical guide to photoacoustic tomography in the life sciences. Nature Methods 13, 627-638 (2016).

[153] Yang, J.-M. et al. Optical-resolution photoacoustic endomicroscopy in vivo. Biomedical Optics Express 6, 918 (2015).

[154] Dong, B., Chen, S., Zhang, Z., Sun, C. \& Zhang, H. F. Photoacoustic probe using a microring resonator ultrasonic sensor for endoscopic applications. Optics Letters 39, 4372-5 (2014).

[155] Bai, X. et al. Intravascular optical-resolution photoacoustic tomography with a $1.1 \mathrm{~mm}$ diameter catheter. PLOS ONE 9, e92463 (2014).

[156] Zackrisson, S., van de Ven, S. M. W. Y. \& Gambhir, S. S. Light In and Sound Out: Emerging Translational Strategies for Photoacoustic Imaging. Cancer Research 74, 9791004 (2014).

[157] Marcu, L. Fluorescence Lifetime Techniques in Medical Applications. Annals of Biomedical Engineering 40, 304-331 (2012).

[158] McGinty, J. et al. Wide-field fluorescence lifetime imaging of cancer. Biomedical Optics Express 1, 627-640 (2010).

[159] Sun, Y. et al. Fluorescence lifetime imaging microscopy for brain tumor image-guided surgery. Journal of biomedical optics 15, 056022 (2010).

[160] Cheng, S. et al. Flexible endoscope for continuous in vivo multispectral fluorescence lifetime imaging. Optics letters 38, 1515-7 (2013).

[161] Sparks, H. et al. A flexible wide-field FLIM endoscope utilising blue excitation light for 
label-free contrast of tissue. Journal of Biophotonics 8, 168-178 (2015).

[162] Sun, Y. et al. Endoscopic fluorescence lifetime imaging for in vivo intraoperative diagnosis of oral carcinoma. Microscopy and microanalysis : the official journal of Microscopy Society of America, Microbeam Analysis Society, Microscopical Society of Canada 19, 791-8 (2013).

[163] Gu, M., Kang, H. \& Li, X. Breaking the diffraction-limited resolution barrier in fiberoptical two-photon fluorescence endoscopy by an azimuthally-polarized beam. Scientific Reports 4, 3627 (2014).

[164] Jermyn, M. et al. A review of Raman spectroscopy advances with an emphasis on clinical translation challenges in oncology. Physics in Medicine and Biology 61, R370-R400 (2016).

[165] Wang, Z. et al. Use of multimode optical fibers for fiber-based coherent anti-Stokes Raman scattering microendoscopy imaging. Optics letters 36, 2967-2969 (2011).

[166] Légaré, F., Evans, C. L., Ganikhanov, F. \& Xie, X. S. Towards CARS Endoscopy. 14, 4427-4432 (2006).

[167] Almond, L. M. et al. Endoscopic Raman spectroscopy enables objective diagnosis of dysplasia in Barrett's esophagus. Gastrointestinal Endoscopy 79, 37-45 (2014).

[168] Jermyn, M. et al. Highly accurate detection of cancer in situ with intraoperative, labelfree, multimodal optical spectroscopy. Cancer Research 77, 3942-3950 (2017).

[169] Georgakoudi, I. et al. Fluorescence, reflectance, and light-scattering spectroscopy for evaluating dysplasia in patients with Barrett's esophagus. Gastroenterology 120, 1620 1629 (2001).

[170] Luthman, A. S., Dumitru, S., Quiros-Gonzalez, I., Joseph, J. \& Bohndiek, S. E. Fluorescence hyperspectral imaging (fHSI) using a spectrally resolved detector array. Journal of Biophotonics 10, 840-853 (2017).

[171] Gaab, M. R. Instrumentation: Endoscopes and equipment. World Neurosurgery 79, S14.e11-S14.e21 (2013).

[172] Saeian, K. et al. Unsedated transnasal endoscopy accurately detects Barrett's metaplasia and dysplasia. Gastrointestinal endoscopy 56, 472-8 (2002).

[173] Sugimoto, H. et al. Surveillance of short-segment Barrett's esophagus using ultrathin transnasal endoscopy. Journal of Gastroenterology and Hepatology (Australia) 30, 4145 (2015).

[174] Tanuma, T., Morita, Y. \& Doyama, H. Current status of transnasal endoscopy using ultrathin videoscope for upper GI tract in the world. Digestive Endoscopy 28, (2016).

[175] Sami, S. S. et al. A randomized comparative effectiveness trial of novel endoscopic techniques and approaches for Barrett's esophagus screening in the community. The American journal of gastroenterology 110, 148-158 (2015).

[176] Moriarty, J. P. et al. Costs associated with Barrett' s esophagus screening in the community: an economic analysis of a prospective randomized controlled trial of sedated versus hospital unsedated versus mobile community unsedated endoscopy. Gastrointestinal Endoscopy (2017).

[177] Iddan, G., Meron, G., Glukhovsky, A. \& Swain, P. Wireless capsule endoscopy. Nature 405, 417-417 (2000).

[178] Fisher, L. R. \& Hasler, W. L. New vision in video capsule endoscopy: current status and future directions. Nature Reviews Gastroenterology \& Hepatology 9, 392-405 (2012). 
[179] Wang, A. et al. Wireless capsule endoscopy. Gastrointestinal Endoscopy 78, 805-815 (2013).

[180] Fernandez-Urien, I., Carretero, C., Armendariz, R. \& Muñoz-Navas, M. Esophageal capsule endoscopy. World Journal of Gastroenterology 14, 5254 (2008).

[181] Ciuti, G., Menciassi, A. \& Dario, P. Capsule Endoscopy: From Current Achievements to Open Challenges. IEEE Reviews in Biomedical Engineering 4, 59-72 (2011).

[182] Liao, Z., Gao, R., Xu, C., Xu, D.-F. \& Li, Z.-S. Sleeve string capsule endoscopy for realtime viewing of the esophagus: a pilot study (with video). Gastrointestinal Endoscopy 70, 201-209 (2009).

[183] Gupta, N. et al. Longer inspection time is associated with increased detection of highgrade dysplasia and esophageal adenocarcinoma in Barrett's esophagus. Gastrointestinal endoscopy 76, 531-8 (2012).

[184] Gkolfakis, P., Tziatzios, G., Dimitriadis, G. D. \& Triantafyllou, K. New endoscopes and add-on devices to improve colonoscopy performance. World Journal of Gastroenterology 23, 3784-3796 (2017).

[185] Gralnek, I. M. et al. Standard forward-viewing colonoscopy versus full-spectrum endoscopy: An international, multicentre, randomised, tandem colonoscopy trial. The Lancet Oncology 15, 353-360 (2014).

[186] Hassan, C. et al. Full-spectrum (FUSE) versus standard forward-viewing colonoscopy in an organised colorectal cancer screening programme. Gut 66, 1949-1955 (2017).

[187] Clancy, N. T. et al. Multispectral image alignment using a three channel endoscope in vivo during minimally invasive surgery. Biomedical optics express 3, 2567-78 (2012).

[188] Winter, C. et al. Automatic adaptive enhancement for images obtained with fiberscopic endoscopes. IEEE Transactions on Biomedical Engineering 53, 2035-2046 (2006).

[189] Waterhouse, D. J., Luthman, A. S. \& Bohndiek, S. E. Spectral band optimization for multispectral fluorescence imaging. 10057, 1005709 (2017).

[190] Luthman, S., Waterhouse, D., Bollepalli, L., Joseph, J. \& Bohndiek, S. A multispectral endoscope based on spectrally resolved detector arrays. 104110A (2017).

[191] Regeling, B. et al. Hyperspectral Imaging Using Flexible Endoscopy for Laryngeal Cancer Detection. Sensors 16, 1288 (2016).

[192] Elter, M., Rupp, S. \& Winter, C. Physically motivated reconstruction of fiberscopic images. Proceedings - International Conference on Pattern Recognition 3, 599-602 (2006).

[193] Rupp, S., Elter, M. \& Winter, C. Improving the accuracy of feature extraction for flexible endoscope calibration by spatial super resolution. Annual International Conference of the IEEE Engineering in Medicine and Biology - Proceedings 6565-6571 (2007).

[194] Rupp, S. et al. Evaluation of spatial interpolation strategies for the removal of combstructure in ber-optic images. Annual International Conference of the IEEE Engineering in Medicine and Biology - Proceedings 3677-3680 (2009).

[195] Lee, C. Y. \& Han, J. H. Integrated spatio-spectral method for efficiently suppressing honeycomb pattern artifact in imaging fiber bundle microscopy. Optics Communications 306, 67-73 (2013).

[196] Han, J.-H., Lee, J. \& Kang, J. U. Pixelation effect removal from fiber bundle probe based optical coherence tomography imaging. Optics Express 18, 7427 (2010).

[197] Wang, P. et al. Fiber pattern removal and image reconstruction method for snapshot 
mosaic hyperspectral endoscopic images. Biomedical Optics Express 9, 780 (2018).

[198] Pelli, D. G. \& Bex, P. Measuring contrast sensitivity. Vision Research 90, 10-14 (2013).

[199] Sturm, M. B. et al. Targeted Imaging of Esophageal Neoplasia with a Fluorescently Labeled Peptide: First-in-Human Results. Science Translational Medicine 5, 184ra61 (2013).

[200] Bird-Lieberman, E. L. et al. Molecular imaging using fluorescent lectins permits rapid endoscopic identification of dysplasia in Barrett's esophagus. Nature medicine 18, 31521 (2012).

[201] Waterhouse, D. J. et al. Design and validation of a near-infrared fluorescence endoscope for detection of early esophageal malignancy. Journal of Biomedical Optics 21, 084001 (2016).

[202] Habibollahi, P. et al. Optical Imaging with a Cathepsin B Activated Probe for the Enhanced Detection of Esophageal Adenocarcinoma by Dual Channel Fluorescent Upper GI Endoscopy. Theranostics 2, 227-34 (2012).

[203] Funovics, M. A. et al. Miniaturized multichannel near infrared endoscope for mouse imaging. Molecular imaging 2, 350-7 (2003).

[204] Realdon, S. et al. In vivo molecular imaging of HER2 expression in a rat model of Barrett's esophagus adenocarcinoma. Diseases of the Esophagus 28, 394-403 (2015).

[205] Nakai, Y., Shinoura, S., Ahluwalia, A., Tarnawski, A. S. \& Chang, K. J. Molecular imaging of epidermal growth factor-receptor and survivin in vivo in porcine esophageal and gastric mucosae using probe-based confocal laser-induced endomicroscopy: Proof of concept. Journal of Physiology and Pharmacology 63, 303-307 (2012).

[206] Li, M. et al. Affinity peptide for targeted detection of dysplasia in Barrett's esophagus. Gastroenterology 139, 1472-80 (2010).

[207] Spectra Viewer | Chroma Technology Corp. Available at: https://www.chroma.com/spectra-viewer. (Accessed: 22nd August 2018)

[208] LI-COR. IRDye ${ }^{\circledR}$ Infrared Dyes. Available at: https://www.licor.com/documents/eukougbp4lxjupcds7pqdjqyom13incs. (Accessed: 22nd August 2018)

[209] Neves, A. A. et al. Detection of early neoplasia in Barrett' s esophagus using lectinbased near-infrared imaging : an ex vivo study on human tissue. Endoscopy 50, 618-625 (2018).

[210] Tjalma, J. J. et al. Molecular-Guided Endoscopy Targeting Vascular Endothelial Growth Factor A for Improved Colorectal Polyp Detection. Journal of nuclear medicine : official publication, Society of Nuclear Medicine 57, 480-486 (2016).

[211] Sheth, R. A. et al. Pilot Clinical Trial of Indocyanine Green Fluorescence-Augmented Colonoscopy in High Risk Patients. Gastroenterology research and practice 2016, 6184842 (2016).

[212] Glatz, J. et al. Near-infrared fluorescence cholangiopancreatoscopy: initial clinical feasibility results. Gastrointestinal endoscopy 79, 664-8 (2014).

[213] Sato, K., Nagaya, T., Choyke, P. L. \& Kobayashi, H. Near infrared photoimmunotherapy in the treatment of pleural disseminated NSCLC: preclinical experience. Theranostics $\mathbf{5}$, 698 (2015). [214] Electron-Multiplying $\quad(\mathrm{EM}) \quad$ Gain.
http://www.qimaging.com/resources/pdfs/emccd_technote.pdf. 
[215] Kaye, P. V. et al. Barrett's dysplasia and the Vienna classification: Reproducibility, prediction of progression and impact of consensus reporting and p53 immunohistochemistry. Histopathology 54, 699-712 (2009).

[216] Joshi, B. P. et al. Multimodal Video Colonoscope for Targeted Wide-Field Detection of Nonpolypoid Colorectal Neoplasia. Gastroenterology 150, 1084-1086 (2016).

[217] Yang, C., Hou, V., Nelson, L. Y. \& Seibel, E. J. Color-matched and fluorescence-labeled esophagus phantom and its applications. Journal of biomedical optics 18, 26020 (2013).

[218] Bays, R., Wagnie, G., Robert, D. \& Braichotte, D. Clinical determination of tissue optical properties by endoscopic spatially resolved reflectometry. 35, (1996).

[219] Bargo, P. R. et al. In vivo determination of optical properties of normal and tumor tissue with white light reflectance and an empirical light transport model during endoscopy. Journal of Biomedical Optics 10, 1-15 (2005).

[220] Holmer, C. et al. Optical properties of adenocarcinoma and squamous cell carcinoma of the gastroesophageal junction. Journal of Biomedical Optics 12, 014025 (2007).

[221] Thueler, P. et al. In vivo endoscopic tissue diagnostics based on spectroscopic absorption, scattering, and phase function properties. Journal of Biomedical Optics 8, 495-503 (2003).

[222] Holmer, C. et al. Optical properties of adenocarcinoma and squamous cell carcinoma of the gastroesophageal junction. Journal of Biomedical Optics 12, 014025 (2007).

[223] Calin, M. A., Parasca, S. V., Savastru, D. \& Manea, D. Hyperspectral Imaging in the Medical Field: Present and Future. Applied Spectroscopy Reviews 49, 435-447 (2014).

[224] Krishnamoorthi, R. \& Iyer, P. G. Molecular biomarkers added to image-enhanced endoscopic imaging: Will they further improve diagnostic accuracy? Best Practice \& Research Clinical Gastroenterology 29, 561-573 (2015).

[225] Fawzy, Y., Lam, S. \& Zeng, H. Rapid multispectral endoscopic imaging system for near real-time mapping of the mucosa blood supply in the lung. Biomedical optics express $\mathbf{6}$, 2980-90 (2015).

[226] Kester, R. T., Bedard, N., Gao, L. \& Tkaczyk, T. S. Real-time snapshot hyperspectral imaging endoscope. Journal of biomedical optics 16, 056005 (2011).

[227] Saito, T. \& Yamaguchi, H. Optical imaging of hemoglobin oxygen saturation using a small number of spectral images for endoscopic application. Journal of Biomedical Optics 20, 126011 (2015).

[228] Johnson, W. R., Wilson, D. W., Fink, W., Humayun, M. \& Bearman, G. Snapshot hyperspectral imaging in ophthalmology. Journal of biomedical optics 12, 014036 (2007).

[229] Mori, M. et al. Intraoperative visualization of cerebral oxygenation using hyperspectral image data: a two-dimensional mapping method. International Journal of Computer Assisted Radiology and Surgery 9, 1059-1072 (2014).

[230] MacKenzie, L. E., Choudhary, T. R., McNaught, A. I. \& Harvey, A. R. In vivo oximetry of human bulbar conjunctival and episcleral microvasculature using snapshot multispectral imaging. Experimental Eye Research 149, 48-58 (2016).

[231] Martinez-Herrera, S. E. et al. Multispectral Endoscopy to Identify Precancerous Lesions in Gastric Mucosa. 8509, 43-51 (2014).

[232] Leavesley, S. J. et al. Hyperspectral imaging fluorescence excitation scanning for colon cancer detection. Journal of Biomedical Optics 21, 104003 (2016). 
[233] Han, Z. et al. In vivo use of hyperspectral imaging to develop a noncontact endoscopic diagnosis support system for malignant colorectal tumors. Journal of Biomedical Optics 21, 016001 (2016).

[234] Kumashiro, R. et al. An integrated endoscopic system based on optical imaging and hyper spectral data analysis for colorectal cancer detection. Anticancer Research 3932, 3925-3932 (2016).

[235] Panasyuk, S. V. et al. Medical hyperspectral imaging to facilitate residual tumor identification during surgery. Cancer Biology and Therapy 6, 439-446 (2007).

[236] Liu, Z., Yan, J., Zhang, D. \& Li, Q.-L. Automated tongue segmentation in hyperspectral images for medicine. Applied optics 46, 8328-8334 (2007).

[237] Akbari, H., Kosugi, Y., Kojima, K. \& Tanaka, N. Wavelet-based compression and segmentation of hyperspectral images in surgery. Lecture Notes in Computer Science (including subseries Lecture Notes in Artificial Intelligence and Lecture Notes in Bioinformatics) 5128, 142-149 (2008).

[238] Hagen, N. \& Kudenov, M. W. Review of snapshot spectral imaging technologies. Optical Engineering 52, 090901 (2013).

[239] Gu, X. et al. Image enhancement based on in vivo hyperspectral gastroscopic images: a case study. Journal of Biomedical Optics 21, 101412 (2016).

[240] Leitner, R. et al. Multi-spectral video endoscopy system for the detection of cancerous tissue. Pattern Recognition Letters 34, 85-93 (2013).

[241] Yang, C. et al. Scanning Fiber Endoscope with multiple fluorescence-reflectance imaging channels for guiding biopsy. 89360R (2014).

[242] Lee, C. M., Engelbrecht, C. J., Soper, T. D., Helmchen, F. \& Seibel, E. J. Scanning fiber endoscopy with highly flexible, $1 \mathrm{~mm}$ catheterscopes for wide-field, full-color imaging. Journal of Biophotonics 3, 385-407 (2010).

[243] Tate, T. H., Keenan, M., Black, J., Utzinger, U. \& Barton, J. K. Ultraminiature optical design for multispectral fluorescence imaging endoscopes. Journal of Biomedical Optics 22, 036013 (2017).

[244] ThermoFisher Fluorescence SpectraViewer.

[245] Bioucas-Dias, J. M. et al. Hyperspectral unmixing overview: Geometrical, statistical, and sparse regression-based approaches. IEEE Journal of Selected Topics in Applied Earth Observations and Remote Sensing 5, 354-379 (2012).

[246] Ezzat, S. et al. The prevalence of pituitary adenomas: A systematic review. Cancer 101, 613-619 (2004).

[247] Theodros, D., Patel, M., Ruzevick, J., Lim, M. \& Bettegowda, C. Pituitary adenomas: historical perspective, surgical management and future directions. CNS oncology 4, 411429 (2015).

[248] Kuo, J. S. et al. Congress of Neurological Surgeons Systematic Review and EvidenceBased Guideline on Surgical Techniques and Technologies for the Management of Patients With Nonfunctioning Pituitary Adenomas. Neurosurgery 79, E536-E538 (2016).

[249] Hazer, D. B. et al. Treatment of acromegaly by endoscopic transsphenoidal surgery: surgical experience in 214 cases and cure rates according to current consensus criteria. Journal of neurosurgery 119, 1467-77 (2013).

[250] Acebes, J. J., Martino, J., Masuet, C., Montanya, E. \& Soler, J. Early post-operative $\mathrm{ACTH}$ and cortisol as predictors of remission in Cushing's disease. Acta 
Neurochirurgica 149, 471-477 (2007).

[251] Bao, X. et al. Extended transsphenoidal approach for pituitary adenomas invading the cavernous sinus using multiple complementary techniques. Pituitary 19, 1-10 (2016).

[252] Berkmann, S., Schlaffer, S. \& Buchfelder, M. Tumor shrinkage after transsphenoidal surgery for nonfunctioning pituitary adenoma. Journal of neurosurgery 119, 1447-52 (2013).

[253] Esquenazi, Y. et al. Endoscopic Endonasal Versus Microscopic Transsphenoidal Surgery for Recurrent and/or Residual Pituitary Adenomas. World Neurosurgery 101, 186-195 (2017).

[254] Verstegen, M. J. T. et al. Intraoperative identification of a normal pituitary gland and an adenoma using near-infrared fluorescence imaging and low-dose indocyanine green. Operative Neurosurgery 12, 260-267 (2016).

[255] Buchfelder, M. \& Schlaffer, S. M. Intraoperative magnetic resonance imaging during surgery for pituitary adenomas: Pros and cons. Endocrine 42, 483-495 (2012).

[256] Akutsu, N., Taniguchi, M. \& Kohmura, E. Visualization of the normal pituitary gland during the endoscopic endonasal removal of pituitary adenoma by narrow band imaging. Acta Neurochirurgica 158, 1977-1981 (2016).

[257] Rigante, M. et al. Preliminary experience with 4K ultra-high definition endoscope: analysis of pros and cons in skull base surgery. Acta Otorhinolaryngologica Italica 237241 (2017).

[258] Sandow, N., Klene, W., Elbelt, U., Strasburger, C. J. \& Vajkoczy, P. Intraoperative indocyanine green videoangiography for identification of pituitary adenomas using a microscopic transsphenoidal approach. Pituitary 18, 613-620 (2015).

[259] Litvack, Z. N., Zada, G. \& Laws, E. R. Indocyanine green fluorescence endoscopy for visual differentiation of pituitary tumor from surrounding structures. Journal of Neurosurgery 116, 935-941 (2012).

[260] Lee, J. Y. K. et al. Folate receptor overexpression can be visualized in real time during pituitary adenoma endoscopic transsphenoidal surgery with near-infrared imaging. Journal of Neurosurgery 1-14 (2017).

[261] Yamada, S. \& Takada, K. Angiogenesis in pituitary adenomas. Microscopy Research and Technique 60, 236-243 (2003).

[262] Jugenburg, M., Kovacs, K., Stefaneanu, L. \& Scheithauer, B. W. Vasculature in Nontumorous Hypophyses, Pituitary Adenomas, and Carcinomas: A Quantitative Morphologic Study. Endocrine pathology 6, 115-124 (1995).

[263] Hide, T., Yano, S., Shinojima, N. \& Kuratsu, J. Usefulness of the indocyanine green fluorescence endoscope in endonasal transsphenoidal surgery. Journal of neurosurgery 122, 1185-1192 (2015).

[264] Highlights | KARL STORZ Endoskope | United Kingdom. Available at: https://www.karlstorz.com/gb/en/highlights-tp.htm. (Accessed: 14th September 2018)

[265] van Dam, G. M. et al. Intraoperative tumor-specific fluorescence imaging in ovarian cancer by folate receptor- $\alpha$ targeting: first in-human results. Nature Medicine 17, 13151319 (2011).

[266] Hoogstins, C. E. S. et al. A novel tumor-specific agent for intraoperative near-infrared fluorescence imaging: A translational study in healthy volunteers and patients with ovarian cancer. Clinical Cancer Research 22, 2929-2938 (2016).

[267] Evans, C. et al. Differential Expression of Folate Receptor in Pituitary Adenomas. 
Cancer Research 4218-4224 (2003).

[268] Larysz, D. et al. Expression of genes FOLR1, BAG1 and LAPTM4B in functioning and non-functioning pituitary adenomas. Folia neuropathologica 50, 277-86 (2012).

[269] Evans, C., Yao, C., LaBorde, D. \& Oyesiku, N. M. Chapter 8 Folate Receptor Expression in Pituitary Adenomas: Cellular and Molecular Analysis. Vitamins and Hormones 79, 235-266 (2008).

[270] Valdés, P. A. et al. Quantitative, spectrally-resolved intraoperative fluorescence imaging. Scientific reports 2, 798 (2012).

[271] di Pietro, M. et al. Detection of dysplasia in Barrett's oesophagus using lectin-based near infra-red molecular imaging: an ex-vivo study on human tissue. in Proceedings of the British Society of Gastroenterology Meeting (2016). 


\section{APPENDIX A. MUSE APPROVAL}

WHS

Health Research Authority

Dr Massimiliano di Pietro

Honorary Consultant Gastroenterologist

Email: hra.approval@nhs.net University of Cambridge

MRC Cancer Unit

University of Cambridge

Hutchison/MRC Research Centre

CB2 OXZ

02 March 2018

Dear Dr di Pietro

\section{Letter of HRA Approval}

Study title:

Prospective pilot cohort study to assess feasibility of multispectral endoscopic imaging for detection of early

IRAS project ID: neoplasia in Barrett's oesophagus

REC reference: 233522

Sponsor

18/NW/0134

Cambridge University Hospitals NHS Foundation Trust and the University of Cambridge

I am pleased to confirm that HRA Approval has been given for the above referenced study, on the basis described in the application form, protocol, supporting documentation and any clarifications noted in this letter.

\section{Participation of NHS Organisations in England}

The sponsor should now provide a copy of this letter to all participating NHS organisations in England.

Appendix B provides important information for sponsors and participating NHS organisations in England for arranging and confirming capacity and capability. Please read Appendix B carefully, in particular the following sections:

- Participating NHS organisations in England - this clarifies the types of participating organisations in the study and whether or not all organisations will be undertaking the same activities

- Confirmation of capacity and capability - this confirms whether or not each type of participating NHS organisation in England is expected to give formal confirmation of capacity and capability. Where formal confirmation is not expected, the section also provides details on the time limit given to participating organisations to opt out of the study, or request additional time, before their participation is assumed.

- Allocation of responsibilities and rights are agreed and documented (4.1 of HRA assessment criteria) - this provides detail on the form of agreement to be used in the study to confirm capacity and capability, where applicable. 
Further information on funding, HR processes, and compliance with HRA criteria and standards is also provided.

It is critical that you involve both the research management function (e.g. R\&D office) supporting each organisation and the local research team (where there is one) in setting up your study. Contact details and further information about working with the research management function for each organisation can be accessed from the HRA website.

\section{Appendices}

The HRA Approval letter contains the following appendices:

- A - List of documents reviewed during HRA assessment

- B - Summary of HRA assessment

\section{After HRA Approval}

The document "After Ethical Review - guidance for sponsors and investigators", issued with your REC favourable opinion, gives detailed guidance on reporting expectations for studies, including:

- Registration of research

- Notifying amendments

- Notifying the end of the study

The HRA website also provides guidance on these topics, and is updated in the light of changes in reporting expectations or procedures.

In addition to the guidance in the above, please note the following:

- HRA Approval applies for the duration of your REC favourable opinion, unless otherwise notified in writing by the HRA.

- Substantial amendments should be submitted directly to the Research Ethics Committee, as detailed in the After Ethical Review document. Non-substantial amendments should be submitted for review by the HRA using the form provided on the HRA website, and emailed to hra.amendments@nhs.net.

- The HRA will categorise amendments (substantial and non-substantial) and issue confirmation of continued HRA Approval. Further details can be found on the HRA website.

Scope

HRA Approval provides an approval for research involving patients or staff in NHS organisations in England.

If your study involves NHS organisations in other countries in the UK, please contact the relevant national coordinating functions for support and advice. Further information can be found through $\underline{\mathbb{R A S}}$.

If there are participating non-NHS organisations, local agreement should be obtained in accordance with the procedures of the local participating non-NHS organisation. 


\section{User Feedback}

The Health Research Authority is continually striving to provide a high quality service to all applicants and sponsors. You are invited to give your view of the service you have received and the application procedure. If you wish to make your views known please use the feedback form available on the HRA website.

\section{HRA Training}

We are pleased to welcome researchers and research management staff at our training days - see details on the HRA website.

Your IRAS project ID is $\mathbf{2 3 3 5 2 2}$. Please quote this on all correspondence.

Yours sincerely

Catherine Adams

Senior Assessor

Email: hra.approval@nhs.net

Copy to: Mr Stephen Kelleher, Sponsor's Representative, Cambridge University Hospitals Trust 
IRAS project ID

233522

\section{Appendix A - List of Documents}

The final document set assessed and approved by HRA Approval is listed below.

\begin{tabular}{|l|l|l|}
\hline Document & Version & Date \\
\hline Covering letter on headed paper [Cover Letter] & 1.0 & 06 February 2018 \\
\hline $\begin{array}{l}\text { Evidence of Sponsor insurance or indemnity (non NHS Sponsors } \\
\text { only) }\end{array}$ & 1 & 01 March 2018 \\
\hline GP/consultant information sheets or letters [MuSE GP Letter] & 1.0 & 01 February 2018 \\
\hline IRAS Application Form [IRAS_Form_06022018] & & 06 February 2018 \\
\hline Participant consent form [Participation consent form] & 1.0 & 01 February 2018 \\
\hline Participant information sheet (PIS) [Participant information sheet] & 1.0 & 01 February 2018 \\
\hline Research protocol or project proposal [MuSE Study Protocol] & 1.0 & 01 February 2018 \\
\hline Summary CV for Chief Investigator (CI) [DrM Di Pietro CV] & 1.0 & 01 February 2018 \\
\hline
\end{tabular}




\section{Appendix B - Summary of HRA Asse ssment}

This appendix provides assurance to you, the sponsor and the NHS in England that the study, as reviewed for HRA Approval, is compliant with relevant standards. It also provides information and clarification, where appropriate, to participating NHS organisations in England to assist in assessing and arranging capacity and capability.

For information on how the sponsor should be working with participating NHS organisations in England, please refer to the, participating NHS organisations, capacity and capabilityand Allocation of responsibilities and rights are agreed and documented (4.1 of HRA assessment criteria) sections in this appendix.

The following person is the sponsor contact for the purpose of addressing participating organisation questions relating to the study:

Mr Stephen Kelleher

E-mail stephen.kelleher@addenbrookes.nhs.uk

Telephone 01223217418

\section{HRA assessment criteria}

\begin{tabular}{|c|c|c|c|}
\hline Section & HRA Assessment Criteria & $\begin{array}{l}\text { Compliant with } \\
\text { Standards }\end{array}$ & Comments \\
\hline 1.1 & $\begin{array}{l}\text { IRAS application completed } \\
\text { correctly }\end{array}$ & Yes & No comments \\
\hline 2.1 & $\begin{array}{l}\text { Participant information/consent } \\
\text { documents and consent } \\
\text { process }\end{array}$ & Yes & No comments \\
\hline 3.1 & Protocol assessment & Yes & No comments \\
\hline 4.1 & $\begin{array}{l}\text { Allocation of responsibilities } \\
\text { and rights are agreed and } \\
\text { documented }\end{array}$ & Yes & $\begin{array}{l}\text { No agreement is required as joint } \\
\text { research managements arrangements } \\
\text { are in place between the Sponsor and } \\
\text { the participating organisation. }\end{array}$ \\
\hline 4.2 & $\begin{array}{l}\text { Insurance/indemnity } \\
\text { arrangements assessed }\end{array}$ & Yes & $\begin{array}{l}\text { The imaging device will be obtained } \\
\text { from Polydiagnost GmbH, Munich } \\
\text { Germany. Polydiagnost will provide } \\
\text { indemnity for the manufacture of the } \\
\text { device. } \\
\text { Where applicable, independent } \\
\text { contractors (e.g. General Practitioners) } \\
\text { should ensure that the professional } \\
\text { indemnity provided by their medical }\end{array}$ \\
\hline
\end{tabular}




\begin{tabular}{|c|c|c|c|c|}
\hline & & & IRAS project ID & 233522 \\
\hline Section & HRA Assessment Criteria & $\begin{array}{l}\text { Compliant with } \\
\text { Standards }\end{array}$ & \multicolumn{2}{|c|}{ Comments } \\
\hline & & & $\begin{array}{l}\text { defence organisation } \\
\text { activities expected of } \\
\text { research study }\end{array}$ & $\begin{array}{l}\text { overs the } \\
\text { em for this }\end{array}$ \\
\hline 4.3 & $\begin{array}{l}\text { Financial arrangements } \\
\text { assessed }\end{array}$ & Yes & No comments & \\
\hline 5.1 & $\begin{array}{l}\text { Compliance with the Data } \\
\text { Protection Act and data } \\
\text { security issues assessed }\end{array}$ & Yes & No comments & \\
\hline 5.2 & $\begin{array}{l}\text { CTIMPS - Arrangements for } \\
\text { compliance with the Clinical } \\
\text { Trials Regulations assessed }\end{array}$ & Not Applicable & No comments & \\
\hline 5.3 & $\begin{array}{l}\text { Compliance with any } \\
\text { applicable laws or regulations }\end{array}$ & Yes & No comments & \\
\hline 6.1 & $\begin{array}{l}\text { NHS Research Ethics } \\
\text { Committee favourable opinion } \\
\text { received for applicable studies }\end{array}$ & Yes & No comments & \\
\hline 6.2 & $\begin{array}{l}\text { CTIMPS - Clinical Trials } \\
\text { Authorisation (CTA) letter } \\
\text { received }\end{array}$ & Not Applicable & No comments & \\
\hline 6.3 & $\begin{array}{l}\text { Devices - MHRA notice of no } \\
\text { objection received }\end{array}$ & Not Applicable & $\begin{array}{l}\text { The device is not inte } \\
\text { commercialisation an } \\
\text { house. }\end{array}$ & $\begin{array}{l}\text { led for } \\
\text { s used in- }\end{array}$ \\
\hline 6.4 & $\begin{array}{l}\text { Other regulatory approvals } \\
\text { and authorisations received }\end{array}$ & Not Applicable & No comments & \\
\hline
\end{tabular}

\section{Participating NHS Organisations in England}

This provides detail on the types of participating NHS organisations in the study and a statement as to whether the activities at all organisations are the same or different.

There is one participating organisation and therefore only one 'site-type'.

If this study is subsequently extended to other NHS organisation(s) in England, an amendment should be submitted to the HRA, with a Statement of Activities and Schedule of Events for the newly participating NHS organisation(s) in England. 


\section{Confirmation of Capacity and Capability}

This describes whether formal confirmation of capacity and capability is expected from participating NHS organisations in England.

This is a single site study sponsored by the site. The R\&D office will confirm to the $\mathrm{Cl}$ when the study can start.

\section{Principal Investigator Suitability}

This confirms whether the sponsor position on whether a PI, LC or neither should be in place is correct for each type of participating NHS organisation in England and the minimum expectations for education, training and experience that Pls should meet (where applicable).

A Principal Investigator is expected at the participating organisation.

GCP training is not a generic training expectation, in line with the HRA/MHRA statement on training

expectations.

\section{HR Good Practice Resource Pack Expectations}

This confirms the HR Good Practice Resource Pack expectations for the study and the pre-engagement checks that should and should not be undertaken

No Honorary Research Contracts, Letters of Access or pre-engagement checks are expected for local staff employed by the participating NHS organisations. Where arrangements are not already in place, research staff not employed by the NHS host organisation un dertaking any of the research activities listed in the research application would be expected to obtain an honorary research contract. This would be on the basis of a Research Passport (if university employed) or an NHS to NHS confirmation of pre-engagement checks letter (if NHS employed). These should confirm enhanced DBS checks, including appropriate barred list checks, and occupational health clearance.

\section{Other Information to Aid Study Set-up}

This details any other information that may be helpful to sponsors and participating NHS organisations in England to aid study set-up.

The applicant has indicated that they intend to apply for inclusion on the NIHR CRN Portfolio. 


\section{APPENDIX B. MAPS APPROVAL}

Ymchwil lechyd

a Gofal Cymru

Health and Care

Research Wales

Mr James Tysome

Consultant ENT and Skull Base Surgeon

Cambridge University Hospitals NHS Trust

Addenbrooke's Hospital

Hills Road

Cambridge

CB2 OQQ

17 August 2018

Dear Mr Tysome

\section{HRA and Health and Care \\ Research Wales (HCRW) \\ Approval Letter}

Study title:

Prospective pilot cohort study to assess feasibility of multispectral endoscopic imaging for delineation of the pituitary gland during transphenoidal surgery for pituitary

IRAS project ID: adenomas.

REC reference:

241616

Sponsor

18/EE/0165

Cambridge University Hospitals NHS Foundation Trust

I am pleased to confirm that HRA and Health and Care Research Wales (HCRW) Approval has been given for the above referenced study, on the basis described in the application form, protocol, supporting documentation and any clarifications received. You should not expect to receive anything further relating to this application.

How should I continue to work with participating NHS organisations in England and Wales? You should now provide a copy of this letter to all participating NHS organisations in England and Wales, as well as any documentation that has been updated as a result of the assessment.

This is a single site study sponsored by the site. The sponsor R\&D office will confirm to you when the study can start following issue of HRA and HCRW Approval.

It is important that you involve both the research management function (e.g. R\&D office) supporting each organisation and the local research team (where there is one) in setting up your study. Contact details of the research management function for each organisation can be accessed here. 
How should I work with participating NHS/HSC organisations in Northern Ireland and Scotland?

HRA and HCRW Approval does not apply to NHS/HSC organisations within the devolved administrations of Northern Ireland and Scotland.

If you indicated in your IRAS form that you do have participating organisations in either of these devolved administrations, the final document set and the study wide governance report (including this letter) has been sent to the coordinating centre of each participating nation. You should work with the relevant national coordinating functions to ensure any nation specific checks are complete, and with each site so that they are able to give management permission for the study to begin.

Please see IRAS Help for information on working with NHS/HSC organisations in Northern Ireland and Scotland.

How should I work with participating non-NHS organisations?

HRA and HCRW Approval does not apply to non-NHS organisations. You should work with your nonNHS organisations to obtain local agreement in accordance with their procedures.

What are my notification responsibilities during the study?

The document "After Ethical Review - guidance for sponsors and investigators", issued with your REC favourable opinion, gives detailed guidance on reporting expectations for studies, including:

- Registration of research

- Notifying amendments

- Notifying the end of the study

The HRA website also provides guidance on these topics, and is updated in the light of changes in reporting expectations or procedures.

\section{Who should I contact for further information?}

Please do not hesitate to contact me for assistance with this application. My contact details are below.

Your IRAS project ID is $\mathbf{2 4 1 6 1 6 . ~ P l e a s e ~ q u o t e ~ t h i s ~ o n ~ a l l ~ c o r r e s p o n d e n c e . ~}$

Yours sincerely,

Steph Blacklock

Senior Assessor

Email: hra.approval@nhs.net

Copy to: Mr Stephen Kelleher, Cambridge University Hospitals NHS Foundation Trust and University of Cambridge, Sponsor Contact

Ms Ruth Holmes, Cambridge University Hospitals NHS Foundation Trust, Lead $R \& D$ Contact 


\section{List of Documents}

The final document set assessed and approved by HRA and HCRW Approval is listed below.

\begin{tabular}{|l|l|l|}
\hline Document & Version & Date \\
\hline Instructions for use of medical device [Device instruction] & 1 & 15 May 2018 \\
\hline IRAS Application Form [IRAS_Form_03052018] & & 03 May 2018 \\
\hline Letter from sponsor [Sponsor support for study] & & 26 March 2018 \\
\hline Other [MAPS peer review] & 1 & 24 April 2018 \\
\hline Participant consent form [MAPS consent form] & 1 & 08 January 2018 \\
\hline Participant information sheet (PIS) & 3 & 24 July 2018 \\
\hline $\begin{array}{l}\text { Referee's report or other scientific critique report [MAPS External } \\
\text { peer review] }\end{array}$ & & 07 March 2018 \\
\hline Research protocol or project proposal [MAPS protocol] & 3 & 05 January 2018 \\
\hline Summary CV for Chief Investigator (CI) [CI CV] & & 03 April 2018 \\
\hline
\end{tabular}




\section{Summary of assessment}

The following information provides assurance to you, the sponsor and the NHS in England and Wales that the study, as assessed for HRA and HCRW Approval, is compliant with relevant standards. It also provides information and clarification, where appropriate, to participating NHS organisations in England and Wales to assist in assessing, arranging and confirming capacity and capability.

\section{Assessment criteria}

\begin{tabular}{|c|c|c|c|}
\hline Section & Assessment Criteria & $\begin{array}{l}\text { Compliant with } \\
\text { Standards }\end{array}$ & Comments \\
\hline 1.1 & $\begin{array}{l}\text { IRAS application completed } \\
\text { correctly }\end{array}$ & Yes & No comments \\
\hline 2.1 & $\begin{array}{l}\text { Participant information/consent } \\
\text { documents and consent } \\
\text { process }\end{array}$ & Yes & No comments \\
\hline 3.1 & Protocol assessment & Yes & No comments \\
\hline 4.1 & $\begin{array}{l}\text { Allocation of responsibilities } \\
\text { and rights are agreed and } \\
\text { documented }\end{array}$ & Yes & $\begin{array}{l}\text { Although formal confirmation of } \\
\text { capacity and capability is not expected } \\
\text { of all or some organisations } \\
\text { participating in this study, and such } \\
\text { organisations would therefore be } \\
\text { assumed to have confirmed their } \\
\text { capacity and capability should they not } \\
\text { respond to the contrary, we would ask } \\
\text { that these organisations pro-actively } \\
\text { engage with the sponsor in order to } \\
\text { confirm at as early a date as possible. } \\
\text { Confirmation in such cases should be } \\
\text { by email to the Cl and Sponsor } \\
\text { confirming participation based on the } \\
\text { relevant Statement of Activities and } \\
\text { information within this letter. }\end{array}$ \\
\hline 4.2 & $\begin{array}{l}\text { Insurance/indemnity } \\
\text { arrangements assessed }\end{array}$ & Yes & No comments \\
\hline 4.3 & $\begin{array}{l}\text { Financial arrangements } \\
\text { assessed }\end{array}$ & Yes & No comments \\
\hline
\end{tabular}

Page 4 of 6 


\begin{tabular}{|c|c|c|c|c|}
\hline & & \multirow{2}{*}{\multicolumn{2}{|c|}{ IRAS project ID }} & \multirow[b]{2}{*}{241616} \\
\hline & & & & \\
\hline Section & Assessment Criteria & $\begin{array}{l}\text { Compliant with } \\
\text { Standards }\end{array}$ & \multicolumn{2}{|c|}{ Comments } \\
\hline 5.1 & $\begin{array}{l}\text { Compliance with the Data } \\
\text { Protection Act and data } \\
\text { security issues assessed }\end{array}$ & Yes & No comments & \\
\hline 5.2 & $\begin{array}{l}\text { CTIMPS - Arrangements for } \\
\text { compliance with the Clinical } \\
\text { Trials Regulations assessed }\end{array}$ & Not Applicable & No comments & \\
\hline 5.3 & $\begin{array}{l}\text { Compliance with any } \\
\text { applicable laws or regulations }\end{array}$ & Yes & No comments & \\
\hline 6.1 & $\begin{array}{l}\text { NHS Research Ethics } \\
\text { Committee favourable opinion } \\
\text { received for applicable studies }\end{array}$ & Yes & No comments & \\
\hline 6.2 & $\begin{array}{l}\text { CTIMPS - Clinical Trials } \\
\text { Authorisation (CTA) letter } \\
\text { received }\end{array}$ & Not Applicable & No comments & \\
\hline 6.3 & $\begin{array}{l}\text { Devices - MHRA notice of no } \\
\text { objection received }\end{array}$ & Not Applicable & No comments & \\
\hline 6.4 & $\begin{array}{l}\text { Other regulatory approvals } \\
\text { and authorisations received }\end{array}$ & Not Applicable & No comments & \\
\hline
\end{tabular}

\section{Participating NHS Organisations in England and Wales}

This provides detail on the types of participating NHS organisations in the study and a statement as to whether the activities at all organisations are the same or different.

This is a single site, device trial.

If this study is subsequently extended to other NHS organisation(s) in England or Wales, an amendment should be submitted, with a Statement of Activities and Schedule of Events for the newly participating NHS organisation(s) in England or Wales.

If chief investigators, sponsors or principal investigators are asked to complete site level forms for participating NHS organisations in England and Wales which are not provided in IRAS, the HRA or HCRW websites, the chief investigator, sponsor or principal investigator should notify the HRA immediately at hra.approval@nhs.net or HCRW at Research-permissions@wales.nhs.uk. We will work with these organisations to achieve a consistent approach to information provision. 


\section{Principal Investigator Suitability}

\begin{tabular}{l} 
This confirms whether the sponsor position on whether a PI, LC or neither should be in place is correct for each \\
type of participating NHS organisation in England and Wales, and the minimum expectations for education, \\
training and experience that PIs should meet (where applicable). \\
\hline It is expected that the Chief Investigator take on Principal Investigator level responsibilities at site. \\
GCP training is not a generic training expectation, in line with the HRA/HCRW/MHRA statement on \\
training expectations.
\end{tabular}

\section{HR Good Practice Resource Pack Expectations}

This confirms the HR Good Practice Resource Pack expectations for the study and the pre-engagement checks that should and should not be undertaken

No HR good practice accesses are expected.

\section{Other Information to Aid Study Set-up}

This details any other information that may be helpful to sponsors and participating NHS organisations in England and Wales to aid study set-up.

The applicant has indicated that they intend to apply for inclusion on the NIHR CRN Portfolio. 